\title{
58th Annual ORCA Congress
}

\author{
July 6-9, 2011, Kaunas, Lithuania
}

\section{Abstracts}

\section{Contents}

Session 1

175 Fluoride

Session 2

183 Clinical Studies

Session 3

191 De- and Remineralization

Session 4

200 Epidemiology

Session 5

209 Erosion

Session 6

217 Diagnostics

Session 7

225 Microbiology

Session 8

232 Caries Risk Assessment, Dental Plaque

240 Author Index 


\section{Caries Research}

\section{Session 1 Fluoride}

\begin{abstract}
1
Validation of Fingernails Fluoride Concentrations as Biomarker of Dental Fluorosis in Children

M.A.R. Buzalaf, ${ }^{\text {* }}$, M.H.C. Rodrigues ${ }^{\mathrm{a}}$, B.S. Almeida ${ }^{\mathrm{a}}$, F.M. Levy ${ }^{\mathrm{a}}$, R. Fukushima ${ }^{\mathrm{a}}$, J.P. Pessan ${ }^{\mathrm{b}}$, G.M. Whitford ${ }^{\mathrm{d}}$, F.C. Sampaio ${ }^{\mathrm{c}}$

mbuzalaf@fob.usp.br

aDepartment of Biological Sciences, Bauru Dental School, University of São Paulo, b Department of Pediatric Dentistry and Public Health, Araçatuba Dental School, São Paulo State University, and ${ }^{\mathrm{CH}} \mathrm{Health}$ Sciences Center, Federal University of Paraíba, Brazil; d Department of Oral Biology, Medical College of Georgia, USA
\end{abstract}

The aim of this study was to validate the use of fingernails fluoride concentrations as an effect biomarker, i.e., as a marker of risk for the development of dental fluorosis. Fifty-six children of both genders (mean age 11.8 years) were examined for dental fluorosis using the TF index (labial surfaces of upper and lower incisors and premolars). Data referring to fingernails fluoride concentrations of these children were obtained from previous studies. Data were analyzed by unpaired t test, ANOVA, bivariate analysis (data dichotomized for fingernails fluoride concentrations $\leq 2$ or $>2 \mu \mathrm{g} / \mathrm{g}$ ) using Fisher's exact test, as well as by multivariate linear regression $(\mathrm{p}<0.05)$. Fingernails fluoride concentrations significantly higher were observed for children that presented dental fluorosis $(4.22 \pm 2.45 \mu \mathrm{g} / \mathrm{g})$ when compared with those who did not present dental fluorosis $(2.24 \pm 1.09 \mu \mathrm{g} / \mathrm{g})$. It was observed a tendency for increasing fingernails fluoride concentrations according with the severity of dental fluorosis. This was confirmed by the significant positive correlation between these variables $\left(\mathrm{r}^{2}=0.40\right)$. Also increasing fingernails fluoride concentrations were observed as water fluoride levels increased. A significantly higher risk of developing fluorosis was observed when fingernails fluoride concentrations were higher than $2 \mu \mathrm{g} / \mathrm{g}$.

\section{* Presenting authors.}

Using $2 \mu \mathrm{g} / \mathrm{g}$ as a threshold, it was seen that fingernails fluoride concentrations have high sensitivity (0.84) but low specificity (0.53) as predictors for dental fluorosis. The high positive predictive value indicated that this biomarker might be useful in public health, since it was able to identify around $80 \%$ of individuals at risk to develop dental fluorosis.

This study was funded by FAPESP and CNPq.

2

\section{Enamel ' $\mathrm{CaF}_{2}$ ' Dissolution and Its Release to Biofilm Fluid under Highly-Cariogenic Challenge and $F$ Dentifrice Use}

L.M.A. Tenuta*, A.A. Del Bel Cury, J.K.B. Fernandes, J.A. Cury

litenuta@fop.unicamp.br

Piracicaba Dental School, University of Campinas, Piracicaba, Brazil

Calcium fluoride-like (' $\mathrm{CaF}_{2}$ ') deposits formed on enamel by acidulated phosphate fluoride (APF) could be a source of fluoride (F) to biofilm fluid to reduce mineral loss during acid challenges, but the maintenance of these F-releasing reservoirs under high cariogenic challenge has not been studied. Also, the effect of concomitant use of F dentifrice has not been explored. This doubleblind, crossover, in situ study evaluated the availability of $F$ to the fluid of biofilms formed on enamel blocks pretreated with APF $(12,300 \mathrm{ppm} \mathrm{F}, \mathrm{pH} 3.5,4 \mathrm{~min})$, under a high cariogenic challenge and the use of $\mathrm{F}$ dentifrice or not. Twelve volunteers wore palatal appliances containing enamel blocks treated on the first day of each phase with APF. Biofilm was allowed to accumulate on the enamel blocks, which were exposed to $20 \%$ sucrose solution $8 \mathrm{X} /$ day. Non-F or F dentifrice $(1,100 \mu \mathrm{g} F / g, \mathrm{NaF})$ was used $3 \times /$ day. After 7 and 14 days, biofilm formed was collected for $\mathrm{F}$ analysis in the fluid. ' $\mathrm{CaF}_{2}$ ' concentration was determined in enamel before, after APF application, and after 14 days in situ. Enamel

\section{KARGER}

Fax +41613061234 E-Mail karger@karger.ch www.karger.com 
' $\mathrm{CaF}_{2}$ ' concentration decreased overtime $(\mathrm{p}<0.05)$, but was still higher than before ATF, and a higher retention was found under use of F dentifrice $(p<0.05)$. Also, F concentration in the fluid of biofilms was significantly higher at 7 days $(21.9 \pm 31.8 \mu \mathrm{M})$ than at 14 days $(5.9 \pm 5.1 \mu \mathrm{M})(\mathrm{p}<0.05)$, and was higher under $\mathrm{F}$ dentifrice use than under non-F dentifrice use $(\mathrm{p}<0.05)$. The results suggest that ' $\mathrm{CaF}_{2}$ ' reservoirs formed on enamel by APF application are maintained even under high cariogenic challenge with consequent release of $\mathrm{F}$ to biofilm fluid, and these effects are enhanced by $\mathrm{F}$ dentifrice use.

Supported by FAPESP (2007/06179-1).

3

\section{Effect of Timing of Fluoride Dentifrice Use on Biofilm Fluoride Concentration and on Enamel Demineralization}

J.A. Cury*, S.C. Kusano, L.M.A. Tenuta, A.A. Del Bel Cury

jcury@fop.unicamp.br

Piracicaba Dental School, University of Campinas, Piracicaba, Brazil

Fluoride (F) interferes with caries process reducing dental demineralization and enhancing remineralization, but the relative importance of each effect is not known. This study evaluated the effect of timing of fluoride dentifrice use on enamel subjected to biofilm accumulation and daily sucrose exposure. In a doubleblind, crossover, 3 legs of 14 days in situ study, 12 volunteers subjected enamel blocks to biofilm accumulation and $20 \%$ sucrose exposure $8 \times /$ day. Three times/day, volunteers brushed with nonF or F $(1,100 \mu \mathrm{g} \mathrm{F} / \mathrm{g}, \mathrm{NaF})$ dentifrices, according to the following groups: $\mathrm{G} 1$ = brushing 3 times/day with non-F dentifrice (control); G2 and G3 = brushing in the morning (before first daily sucrose exposure) or after dinner (after last sucrose exposure) with the F dentifrice, respectively, and otherwise with non-F dentifrice. After each phase, biofilm samples were collected before morning brushing and first sucrose exposure of the day, and in the evening after the last sucrose exposure and before the last brushing of the day for F analysis in the fluid and solids. On enamel blocks, demineralization was determined by change of surface hardness. F concentration in biofilm fluid collected on fasting was not significantly different among groups $(p>0.05)$. A significantly higher $\mathrm{F}$ concentration was found in the biofilm solids in group brushing with $\mathrm{F}$ dentifrice at night when compared to the control $(p<0.05)$. Significantly higher demineralization $(\mathrm{p}<0.05)$ was found in the control group $(72.0 \pm 20.8 \%)$, but no significant difference was reached between groups brushing with F dentifrice in the morning $(41.6 \pm 21.5 \%)$ or at night $(30.0 \pm$ $15.8 \%)(\mathrm{p}>0.05)$. The results suggest that brushing with $\mathrm{F}$ dentifrice before sleeping is able to enhance $\mathrm{F}$ retention in the biofilm solids and could as effective as to control caries than brushing in the morning.

FAPESP 2009/07019-3.

\section{4 \\ Fluoride Release to Plaque Fluid from Enamel Treated with Fluoride Gel and Varnish}

\author{
A.A. Del Bel Cury*, R.V. Cerezetti, L.M. Tenuta, T.J. Oliveira, J.A. Cury \\ altcury@fop.unicamp.br \\ Piracicaba Dental School, University of Campinas, Piracicaba, \\ Brazil
}

Calcium fluoride-like (' $\mathrm{CaF}_{2}$ ') products formed on enamel by fluoride (F) gel or varnish treatments could be a source of $\mathrm{F}$ to plaque fluid, but the effect of time after the application has not been explored. Twelve volunteers wore in situ, for 3 phases, a palatal appliance containing a test plaque of $S$. mutans in contact with bovine enamel blocks not treated (control) or treated with acidulated phosphate fluoride (APF) gel ( $\left.\mathrm{DFL}^{\circledR}, 1.23 \% \mathrm{~F}, \mathrm{pH} 3.5\right)$ or F varnish (Duraphat ${ }^{\circledR}, 5 \% \mathrm{NaF}$ ), either immediately after application or after aging. APF gel was applied for 4 min and the applied varnish was kept for $24 \mathrm{~h}$ under continuous flow of artificial saliva before being removed with acetone. Aging was made in artificial saliva for 7 days. The test plaque was collected after 30 min of appliance use for analysis of fluid $\mathrm{F}$ concentration. Enamel ' $\mathrm{CaF}_{2}$ ' concentration was determined in fresh and aged blocks. Fluoride concentration on enamel of control, APF or varnish groups were, respectively, $0.3 \pm 0.1,42.3 \pm 24.0$ and $25.7 \pm$ $2.8 \mu \mathrm{g} \mathrm{F} / \mathrm{cm}^{2}$ when not aged, and $0.3 \pm 0.1,1.7 \pm 0.7$ and $5.5 \pm$ 2.1 after aging; accordingly, in the plaque fluid, F concentrations were $6.8 \pm 2.7,408.4 \pm 100.5$ and $672.5 \pm 95.3 \mu \mathrm{M}$ for blocks not aged, and $7.0 \pm 1.3,26.9 \pm 9.7$ and $61.9 \pm 20.1 \mu \mathrm{M}$ for aged blocks. For both variables, in fresh blocks APF and varnish groups did not differ from each other $(p>0.05)$, but after aging significant differences were found among the three groups $(\mathrm{p}<0.05)$. A significant correlation $(\mathrm{r}=0.92, \mathrm{p}<0.0001)$ was found between ' $\mathrm{CaF}_{2}$ ' concentration on enamel and $\mathrm{F}$ released to the fluid. The results suggest that both professional $\mathrm{F}$ treatments are able to increase $\mathrm{F}$ concentration in plaque fluid and that this effect is maintained even after aging.

Supported by FAPESP 2008/01727-3.

\section{5 \\ Effect of Fluoridated Milk with Different Frequency of Consumption on Demineralisation of Enamel in situ}

\author{
M. Malinowski*, M. Duggal, S. Strafford, J. Toumba \\ M.Malinowski@leeds.ac.uk \\ Paediatric Dentistry, Leeds Dental Institute, UK
}

Aim: To evaluate the effect of fluoridated milk with varying frequency of consumption on enamel demineralisation under cariogenic challenge. Methods: 25 subjects were recruited to a controlled, randomised, 3-arm crossover, single-blinded clinical study. Subjects wore intra-oral appliances containing enamel slabs for 21 days per study arm. The cariogenic challenge comprised 5 dippings ( 2 min each) per day in 12\% sucrose. Subjects also dipped their appliances in $50 \mathrm{ml}$ of $5.0 \mathrm{ppm} \mathrm{F}$ milk, either once, twice or once every other day for $5 \mathrm{~min}$ and drank $200 \mathrm{ml}$ 
of $5.0 \mathrm{ppm}$ fluoridated milk either once, $100 \mathrm{ml}$ of $5.0 \mathrm{ppm}$ fluoridated milk twice or $200 \mathrm{ml}$ of $5.0 \mathrm{ppm}$ F fluoridated milk once every other day immediately on reinserting their appliances. At the end of the study, slabs were analysed for Knoop microhardness to assess changes in mineralisation. Results: Enamel was softened in all groups after 21 days. Percentage difference of means was used to compare between groups. The reduction in softness of enamel was highly significant for the groups with frequency of consumption once and twice per day compared to baseline ( $\mathrm{p}<0.001$, Wilcoxon test) and for once every other day $(\mathrm{p}<$ 0.05 , Wilcoxon test). Between groups this reduction in softness of enamel was significant for the once per day group compared to twice per day ( $p<0.05$, Wilcoxon test). Conclusions: In this RCT model, using SMH, drinking $200 \mathrm{ml}$ of 5.0 ppm F once per day showed a significantly protective effect on reduction of enamel demineralisation/softness than drinking $100 \mathrm{ml}$ of $5.0 \mathrm{ppm} \mathrm{F}$ twice per day.

Supported by a grant from The Borrow Foundation.

\section{6}

\section{Fluoride Uptake from Toothpastes with Different Relative Enamel Abrasivity}

\section{F.J. Wegehaupt*, V. Elmazi, B. Sener, T. Attin, T. Imfeld}

Florian.Wegehaupt@zzm.uzh.ch

Clinic for Preventive Dentistry, Periodontology and Cariology,

University of Zurich, Switzerland

This in vitro study aimed to determine the influence of the relative enamel abrasivity (REA) of fluoridated toothpaste on $\mathrm{KOH}$-soluble and structurally bound fluoride uptake. Bovine enamel samples (drilling cores: diameter $3 \mathrm{~mm}$; embedded in acrylic resin; ground flat) were randomly allocated to 6 groups ( $\mathrm{n}=36$ per group). Groups $1-3$ were treated with $\mathrm{NaF}$ toothpaste and groups 4-6 with AmF toothpaste (1,500 ppm F, respectively). REA was 2 in groups 1 and 4, 6 in groups 2 and 5, and 9 in groups 3 and 6. Twice a day 18 samples of each group were immersed $(2 \mathrm{~min})$ in a slurry (toothpaste:artificial saliva $=1: 3)$ for 5 days, while the remaining samples were brushed with respective slurry (2.5 N; 60 strokes/min; $2 \mathrm{~min})$. All samples were stored at $37^{\circ} \mathrm{C}$ and $100 \%$ humidity (moist chamber). After five days, the amount of $\mathrm{KOH}$-soluble and structurally bound (acidic biopsy) fluoride was determined and compared by Scheffe's post hoc tests. The $\mathrm{NaF}$ toothpastes showed no difference in the uptake of both kinds of fluorides, irrespective of REA and mode of application (immersion or brushing). Only for the NaF toothpaste with REA-6 was the amount of $\mathrm{KOH}$-soluble fluoride significantly higher after brushing ( $54.36 \pm 36.9$ vs. $15.9 \pm 12.6 \mu \mathrm{g} / \mathrm{cm}^{2}$ (immersed)). For the AmF groups, $\mathrm{KOH}$-soluble and structurally bound fluoride was significantly higher when the samples were brushed (e.g. REA-2: $250.5 \pm 113.7$ vs. $118.3 \pm 108.4 \mu \mathrm{g} / \mathrm{cm}^{2}$ (immersed) and $778.0 \pm 249.1$ vs. $373.0 \pm 151.9 \mu \mathrm{g} / \mathrm{cm}^{3}$ (immersed)). Furthermore, in the REA-2 groups the amounts of $\mathrm{KOH}$-soluble fluoride (brushed or immersed) and structurally bound fluoride (brushed) were significantly higher compared with the other groups. REA dependency of $\mathrm{KOH}$-soluble and structurally bound fluoride was found only for the AmF toothpaste. Mode of application had an influence for AmF toothpaste.
Dental Fluorosis Prevalence and Its Associated Risk Factors in 12- to 18-Year-Old Children in Gaza Strip

\author{
L. Abuhaloob ${ }^{\mathrm{a}, *}$, Y. Abed $^{\mathrm{b}}$ \\ lamies_a@yahoo.com \\ aPalestinian Ministry of Health and 'bShool of Public Health, \\ Al-Quds University, Palestine
}

The aim of this study was to estimate the prevalence and severity of dental fluorosis (DF) in Gaza Strip (GS), to explore the possible associated DF risk factors, and to examine the association between DF and fluoride concentration in the Municipals Wells of Drinking Water (MWDW) which supply children's homes. A cross-sectional study recruited stratified cluster random sample (350, 12- to 18-year-old children) from the five governorates in GS. Children, resident in the same area since birth, and their mothers were interviewed to complete closed and open-ended questionnaires. Prevalence and severity of DF were determined using Thylstrup-Fejerskov index (TFI). Fluoride concentration of MWDW is reported. The Odds Ratio (95\% confidence interval $(\mathrm{CI})$ ) and multiple regression $(\mathrm{p}<0.05)$ were used to examine the association between variables. The prevalence of DF was $78 \%$ (63.4\% was questionable to moderate (TFI score 1-4) and $14.6 \%$ was severe (TFI score 5-8)). The following factors significantly increased the DF risk: use of more than a pea size of fluoridated toothpaste $(\mathrm{OR}=4.50$, CI 1.92-10.61), swallowing fluoridated toothpaste ( $\mathrm{OR}=2.73$, CI 1.17-6.49), live in houses near a main $\operatorname{road}(\mathrm{OR}=2.36, \mathrm{CI} 1.07-5.38)$ and use open fire as heating system during winter $(\mathrm{OR}=3.08$, CI 1.40-6.96). The F concentration in $57.1 \%$ of MWDW was $>1$ ppm. Prevalence of DF was not significantly higher among children receiving MWDW with $>1 \mathrm{ppm} \mathrm{F}$ $(\mathrm{OR}=1.15$, CI 0.66-1.98). Multiple regression analysis revealed that swallowing toothpaste remained a significant factor $(\mathrm{p}<$ 0.005 ). Preventive interventions aim to control DF problem and high fluoride exposure in developing countries should consider all risk factors associated with this condition.

\section{8 \\ Fluoride Intake from Toothpaste Ingested by 4- to 6-Year-Old Children in England}

\author{
F.V. Zohooria, *, N. Omid a , R. Duckworth', A. Batterham ${ }^{\text {, }}$, \\ W.T. O'Hare ${ }^{\mathrm{b}}$, A. Maguire
}

v.zohoori@tees.ac.uk

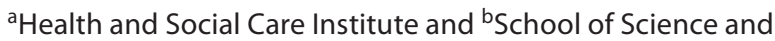
Engineering, Teesside University, Middlesbrough, 'School of Dental Sciences, Newcastle University, Newcastle upon Tyne, UK

Fluoridated toothpaste can be an important source of fluoride (F) intake in young children because they are likely to ingest it during toothbrushing. The aim of this study, which was a part of a larger study to evaluate the validity of dietary methods for F assessment in children, was to investigate the amount of ingested $\mathrm{F}$ per toothbrushing. Sixty-one children, aged 4-6 years, recruited through schools in a fluoridated area in the north-east of England 
were visited twice at their homes and asked to brush their teeth according to their usual habits. The amount of toothpaste (and thus the amount of F) dispensed was measured. Following brushing, all expectorated saliva, rinse water and residual toothpaste from the brush was collected and the amount of recovered $\mathrm{F}$ measured using a F-ion-selective-electrode. Repeated-measure ANOVA was used to compare $F$ ingestion per brushing at the first and second visits. The mean (SD) weight of toothpaste dispensed was $0.72(0.50) \mathrm{g}$ at the first visit and $0.63(0.34) \mathrm{g}$ at the second visit. The corresponding mean (SD) amounts of $\mathrm{F}$ ingested per brushing were $0.018(0.020)$ and $0.016(0.014) \mathrm{mg} / \mathrm{kg}$ body weight, respectively. There were no statistically significant differences between the amounts of toothpaste dispensed and the amounts of $\mathrm{F}$ ingested per brushing at the first and second visits. However, there was a positive statistically significant correlation $(r=0.73$, $p<0.001$ ) between the weight of toothpaste dispensed per brushing and the amount of $\mathrm{F}$ ingested per brushing. Mean combined (average of both brushing sessions) value for the amount of $\mathrm{F}$ ingested per brushing was $0.017 \mathrm{mg} / \mathrm{kg}$ body weight with a very wide range from 0.002 to $0.080 \mathrm{mg} / \mathrm{kg}$ body weight. The present study confirms that more $\mathrm{F}$ is ingested when more $\mathrm{F}$ (toothpaste) is applied.

Supported by grants from Teesside University Research Doctoral Scholarships and The Borrow Foundation.

\section{9 \\ Factors Associated with Fluoride Concentrations in Whole and Parotid Ductal Saliva \\ J.P. Pessan ${ }^{\mathrm{a}, *}$, R. Fukushima ${ }^{\text {b }}$, F.C. Sampaioc, M.A.R. Buzalaf ${ }^{\mathrm{b}}$ \\ mbuzalaf@fob.usp.br \\ aDepartment of Pediatric Dentistry and Public Health, \\ Araçatuba Dental School, São Paulo State University, \\ ${ }^{b}$ Department of Biological Sciences, Bauru Dental School, University of São Paulo, and 'Health Sciences Center, Federal \\ University of Paraíba, João Pessoa, Brazil}

There are still uncertainties regarding the use of whole and parotid ductal saliva as indicators of chronic exposure to fluoride. This study evaluated the effect of water fluoride concentration, age, gender, geographical area (northeast $=$ warmer weather, lower income/southeast = colder weather, higher income) and localization (urban/rural) on fluoride concentrations in whole and parotid ductal saliva. Subjects $(n=300)$, aged 3-7, 14-20, 30-40 and 50-60 years, from five communities (A-E) with different fluoride concentrations in the drinking water participated in the study. Two samples of drinking water and parotid and whole saliva were collected for each subject, and analyzed for fluoride using appropriate electrode techniques. Results were analyzed by ANOVA, Tukey's test, and multivariate linear regression analysis $(\mathrm{p}<0.05)$. Mean water $\mathrm{F}$ concentrations $( \pm \mathrm{SE}, \mathrm{mg} / \mathrm{l}, \mathrm{n}=60)$ were $0.09 \pm$ 0.01a, $0.15 \pm 0.01 \mathrm{a}, 0.66 \pm 0.01 \mathrm{~b}, 0.72 \pm 0.02 \mathrm{~b}$, and $1.68 \pm 0.08 \mathrm{c}$ for A-E, respectively. Mean F concentrations $( \pm$ SE, $\mathrm{mg} / \mathrm{l}, \mathrm{n}=15$ ) ranged between $0.014 \pm 0.002(\mathrm{~A}, 3-7$ years) and $0.297 \pm 0.057$ (D, 14-20 years) for whole saliva and $0.009 \pm 0.001$ (C, 30-40 years) and $0.284 \pm 0.038$ (E, 50-60 years) for parotid saliva. Fluoride concentrations in whole saliva were significantly affect- ed by water fluoride concentration, localization (rural $<$ urban) and geographical area (northeast $>$ southeast), while ductal saliva fluoride levels were affected by water fluoride concentration, age and localization (rural $>$ urban). Geographical area $(\beta=0.78$, adjusted $\left.\mathrm{r}^{2}=0.50, \mathrm{p}<0.001\right)$ and water fluoride concentration $(\beta=$ 0.57 , adjusted $\mathrm{r}^{2}=0.52, \mathrm{p}<0.001$ ) exerted most influence in whole and ductal saliva $\mathrm{F}$ concentrations, respectively. Therefore, parotid ductal saliva seems to be a more appropriate biomarker of fluoride exposure, and factors like age and localization should also be considered when using this biomarker.

FAPESP (grants 04/15417-5, 03/03660-0, 03/03662-2, 02/07875-8).

\section{0 \\ Total Fluoride Intake and Urinary Fluoride Excretion of 4- to 6-Year-Olds in a Fluoridated Area, UK \\ N. Omid ${ }^{\mathrm{a}, *}$, A.M. Batterham ${ }^{\text {, }}$,W.T. O'Hare ${ }^{\mathrm{a}}$, A. Maguire ${ }^{\mathrm{b}}$, F.V. Zohoori ${ }^{\mathrm{a}}$ \\ G7128303@tees.ac.uk \\ ${ }^{\mathrm{a} T e e s s i d e ~ U n i v e r s i t y, ~ M i d d l e s b r o u g h, ~ a n d ~}{ }^{\mathrm{b}}$ Newcastle University, Newcastle upon Tyne, UK}

Information regarding total daily fluoride (F) intake (TDFI) and the proportion of the TDFI excreted in urine is essential for taking appropriate actions to minimise enamel fluorosis while maximising the decay preventive benefits of $F$. The aims of this study were to determine (a) TDFI and (b) fractional urinary F excretion (FUFE) in children who had lived continuously in fluoridated areas of the north east of England. As part of a larger study to evaluate the validity of dietary methods for assessment of $\mathrm{F}$ exposure in children, 61 healthy 4 - to 6-year-old children were recruited from schools in Newcastle (mean F concentration in home supply water; $0.97(0.02) \mu \mathrm{g} / \mathrm{ml})$. For each child, dietary information was collected using a ' 3 -day food diary', and information on toothbrushing habits and collection of expectorate from toothbrushing to estimate $\mathrm{F}$ intake from toothpaste ingestion. The F concentration of toothbrushing and urine samples was measured directly using F-ion-selective electrode (Model 720, Orion) after adding TISABIII. The TDFI was calculated from dietary intake and $\mathrm{F}$ ingestion during toothbrushing. Urinary F excretion (UFE) in $24 \mathrm{~h}$ urine was also measured. The FUFE was calculated from UFE and TDFI [FUFE $=($ UFE/TDFI $) \times 100]$. Mean (SD) TDFI was $0.052(0.030) \mathrm{mg} / \mathrm{kg}$ bw/day of which $46 \%$ was contributed by F intake from toothpaste ingestion. Mean (SD) UFE was 0.018 (0.009) $\mathrm{mg} / \mathrm{kg}$ bw/day. There was a positive significant correlation between UFE and TDFI $(\mathrm{r}=0.52 ; \mathrm{p}<0.001)$. The mean (SD) FUFE was $41 \%$ (26\%). The findings of this study suggest that despite living in a fluoridated area, more than half of the children are ingesting daily amounts of $\mathrm{F}$ below the recommended daily intake of 0.05-0.07 mg/kg body weight [J Dent Res 1992;71:1228-1237] for effective protection against caries.

Supported by grants from University of Teesside Research Doctoral Scholarships and The Borrow Foundation. 


\section{1 \\ Effectiveness of a $\mathbf{5 0 0}$ ppm $\mathrm{F}^{-}$Containing Toothpaste Compared to a 250 ppm F- Containing Toothpaste in 3-Year-Old Children}

\author{
A. Schreiber ${ }^{\mathrm{a}, *}$, T. Marthaler $^{\mathrm{b}}$, J. Rotgans ${ }^{\mathrm{c}}$ \\ schreiber.angelik@snafu.de \\ aDental Public Health Main-Kinzig-Kreis, Germany; \\ bPreventive Dentistry and Oral Epidemiology, University of \\ Zurich, Switzerland; 'Department of Conservative Dentistry, \\ RWTH University Aachen, Germany
}

The aim of the double blind test-control-study was to reject $(\mathrm{p}<0.05$; power 0.8$)$ the hypothesis 'use of $500 \mathrm{ppm} \mathrm{F}^{-}$toothpaste from age three to five years shows no difference in caries increment compared to a $250 \mathrm{ppm} \mathrm{F}^{-}$toothpaste'. 11 kindergartens were randomly selected in Berlin-H'hausen and randomly allocated to the 250 or 500 ppm F$^{-}$toothpaste. Between 1997 and 2004>645 children brushed their teeth with the study toothpaste $1 \times$ daily in the kindergartens, and unsupervised 1-2 $\times$ daily at home. Caries was scored by visual examinations of the teeth dried with compressed air by a single examiner (intra-examiner reliability with 181 children: kappa 0.81 for established lesions). 311 children used the 250 ppm toothpaste and 334 the 500 ppm toothpaste. The 2 year mean net primary molar caries increment as $\mathrm{dmfs}( \pm \mathrm{SD})$ was $3.6( \pm 6.6)$ for the $250 \mathrm{ppm}$ toothpaste and $2.3( \pm 4.1)$ for the $500 \mathrm{ppm}$ toothpaste. Data were analysed using stepwise multivariate logistic regression (Macintosh SPSS 10.0) with 'presence of net caries increment of primary molars' (250 ppm: $50 \%$ CI $43-54 \%$; 500 ppm: $41 \%$ CI 36-46\%) as the dependent variable. 'Fluoride tablets at home from birth up to age three' (41\%), whether children got fluoride tablets further on or not, and 'caries experience at age three' (21\%) were all significant $(p<0.001)$ independent variables. For 'children without caries at age three' fluoride tablets remained significant. For 'children with caries at age three', fluoride content of toothpaste was the only factor ( $p=0.023$ ) explaining the dependent variable. Nevertheless, because of the caries protective tendency for children with high caries risk and only negligible increase in urinary fluoride excretion (to be published separately), toothpaste with $500 \mathrm{ppm} \mathrm{F}^{-}$can be recommended for children from age three. There is need for research about feasibility and effectiveness of toothpaste with $500 \mathrm{ppm}$ fluoride for children under age three.

GABA INTERNATIONAL, Therwil, Switzerland.

\section{2 \\ Total Daily Fluoride Intake and Fractional Urinary Excretion of Fluoride in 6- to 7-Year-Olds}

A. Maguire ${ }^{\mathrm{a}, *}$, R. Walls ${ }^{\mathrm{a}}$, L. Teasdale ${ }^{\mathrm{b}}$, D. Landes ${ }^{\mathrm{b}}$, N. Omid $^{\mathrm{c}}$, N. Steen ${ }^{\text {a }}$, P. Moynihan a , J. Lloyd ${ }^{\text {a }}$, V. Zohooric

A.Maguire@ncl.ac.uk

${ }^{a}$ Newcastle University, ${ }^{b}$ County Durham Primary Care Trust, and

'Teesside University, UK

Background: Urine is the prime vehicle for the body's excretion of ingested fluoride $(\mathrm{F})$ but the relationship between $\mathrm{F}$ intake and excretion is complex and the derived fractional urinary ex- cretion of fluoride (FUEF) aids its understanding in different age groups. Aim: To investigate the correlation between total daily fluoride intake (TDFI) and FUEF in 6- to 7-year-old children. Methods: Children aged 6-7 years were recruited in non-fluoridated (Non-F) and naturally-fluoridated (Natural-F) areas in north east England. TDFI from diet and toothbrushing was assessed using $\mathrm{F}$ analysis of a duplicate-plate and toothbrushing expectorate. Daily urinary fluoride excretion (DUFE) was measured on the same day as duplicate diet collection. F concentration of urine, water and drink samples was measured directly using an F-ion-selective electrode after adding TISABIII. Duplicate food samples were measured for F content using the silicon-facilitated diffusion method. FUEF was calculated as the ratio between DUFE and TDFI. Pearson correlation and regression analysis were used to investigate the relationship between TDFI and FUEF. Results: Thirty-three children completed the study; 21 receiving Non-F water $($ mean $=0.30( \pm 0.12) \mathrm{mg} \mathrm{F} / \mathrm{l})$ and 12 receiving Natural-F water $($ mean $=1.06( \pm 0.11) \mathrm{mg} F / \mathrm{l})$ at school. Mean (SD) TDFI was $1.707(0.8) \mathrm{mg} /$ day for the Natural-F group and 0.945 (0.62) $\mathrm{mg} /$ day for the Non-F group. F intake from toothpaste ingestion represented $59 \%$ and $56 \%$ of TDFI for children in Natural$\mathrm{F}$ and Non-F groups, respectively. Mean (SD) DUFE was 0.393 (0.209) and 0.297 (0.131) mg/day for the Natural-F and Non-F groups, respectively. FUEF was slightly lower in the Natural-F (30\%) compared with the Non-F group (40\%). The Pearson correlation coefficient between TDFI and FUEF was -0.63 ( $\mathrm{p}<$ 0.001 ). Conclusion: There was a statistically significant negative correlation between FUEF and TDFI in 6- to 7-year-olds.

This study was supported by a grant from The Borrow Foundation.

13

\section{In vitro Effect of an Amine Fluoride Mouthrinse and Toothpaste on Dentinal Tubules}

\section{R. Chalas ${ }^{\mathrm{a},}$ * T. Bachanek ${ }^{\mathrm{a}}$, J. Nowak ${ }^{\mathrm{b}}$, A. Kuczumow ${ }^{\mathrm{b}}$ \\ drrzenia@wp.pl \\ aDepartment of Conservative Dentistry, Medical University of Lublin, and bepartment of Chemistry, Catholic University of Lublin, Poland}

The aim of this study was to evaluate the ability of a mouthrinse and a toothpaste containing the amine fluoride, Olaflur, to promote the occlusion of dentine tubules in an in vitro model. Three extracted unerupted human third molars were cut into two $1.5 \mathrm{~mm}$ dentinal discs with a diamond saw, and etched. The discs were allocated to the following groups: control (artificial saliva), Elmex ${ }^{\circledR}$ Sensitive Plus dental rinse (ESDR, 250 ppm F- from Olaflur and potassium fluoride) and Elmex ${ }^{\circledR}$ Sensitive Plus toothpaste (ESTP, 1,400 $\mathrm{ppm} \mathrm{F}^{-}$from Olaflur). The rinse was applied using $10 \mathrm{ml}$ diluted with $10 \mathrm{ml}$ artificial saliva for 2, 20 and 120 min. The toothpaste was applied by brushing for 2 min with an electric toothbrush using a pea-sized amount. Afterwards the discs were rinsed with distilled water and dried. The samples were prepared for scanning electron microscopy (SEM) and image analysis. Comparison of the test discs comparing with the control discs demonstrated visually a blocking of $80 \pm 5 \%$ of the dentine 
tubules by ESTP. Small and large deposits on the dentine surface were diffused as well as localized around and inside the tubular openings. The shape of the deposits was mainly spherical. After the mouthrinse application a slightly different shape of deposits was more frequent. The presence of cylindrical structures inside the dentinal tubules was found. The most pronounced effect was visible after $120 \mathrm{~min}$ of ESDR application: $70 \pm 4 \%$ tubules occluded in comparison with $20 \pm 5 \%$ after 2 min and $40 \pm 7 \%$ after $20 \mathrm{~min}$. However, spherical shape structures were also observed on the dentine surface of discs stored in the mouthrinse. The results from this in-vitro study indicate that the fluoride components of the toothpaste and mouthrinse have occluding properties on dentine tubules. The shape of deposits seems to vary depending on which product is applied, paste or rinse.

Supported by GABA.

\section{4}

Fluoridation of Public Water Supplies in the State of Rio de Janeiro, Brazil

S. Groisman ${ }^{\mathrm{a}, *}$, J.J. Soet ${ }^{\mathrm{d}}$, M. Buzalaf ${ }^{\mathrm{b}}$, C. Loivos $^{c}$, L.T. Grizzo ${ }^{\mathrm{b}}$, A.R. Olival', M. Corvino ${ }^{c}$

sonia@dentistas.com.br

${ }^{\text {aS }}$ Chool of Dentistry, Federal University of Rio de Janeiro, ${ }^{\text {bB Bauru }}$ Dental School, University of São Paulo, and 'School of Dentistry, Federal University of Niterói, Brazil; ${ }^{d}$ Academic Center for

Dentistry Amsterdam, The Netherlands

The fluoridation of public water supplies was recognised by the World Health Organization as one of the ten most relevant measures in public health in the last century. In countries like Brazil, this measure is important to help to improve the dental situation in a socially deprived population. In order that this measure is effective for caries control it is important to maintain adequate fluoride levels in the water and external monitoring has been shown to be adequate for this purpose. Seventy-two water samples were collected from the public supply in 11 municipalities of the state of Rio de Janeiro, Brazil, in May and June 2010. The samples were collected directly from the taps in $50 \mathrm{ml}$ appropriately labeled polyethylene bottles and analysed for fluoride in duplicate, using an electrode (Orion 9609) coupled to a potentiometer. After analyses, samples were classified according to their fluoride concentration (low: $<0.7 \mathrm{ppm}$; adequate: $0.7-0.9$ ppm; high: $>0.9 \mathrm{ppm}$ ). The mean fluoride concentration found was $0.52 \pm 0.28 \mathrm{ppm}$. Approximately $26 \%$ of the samples were considered adequate, while $74 \%$ were regarded as low. The municipality with the highest percentage of adequate samples was the capital, Rio de Janeiro (76\%). The municipalities of Paracambi, Lamb and Areal had all samples considered low. Only $4.2 \%$ of the analysed samples were $>0.9 \mathrm{ppm}$. From the results, it was observed that most of the samples analysed had fluoride levels below those regarded as optimum by the WHO for the control of dental caries. These results reinforce the need of implementation of external monitoring of the fluoridation of public water supplies to assure that the water fluoride concentrations are maintained in the optimum levels.
15

\section{Equilibrium Salivary Fluoride as an Outcome Measure for Oral Health Promotion}

\author{
H. Whelton*, R. Kingston, T. Beecher, A. Grannell \\ h.whelton@ucc.ie \\ Oral Health Services Research Centre, University College Cork, \\ Cork, Ireland
}

Aim: To explore the impact of different approaches to oral health promotion on salivary fluoride levels. Methods: Eleven- to thirteen-year-old children in six schools were recruited to three groups. Each group was comprised of children from a single girl's school and a single boys school. Group 1 received a baseline visit from the dental team but no intervention (control group). Group 2 received a dental health education programme aimed at encouraging twice daily tooth brushing at home supported with brushing diary for home use. Group 3 received the same programme as group 2 plus supervised daily school based tooth brushing for a four week period. Fluoride levels were measured in resting saliva $18 \mathrm{~h}$ after the last brushing with fluoride toothpaste (equilibrium salivary fluoride level, 18-ESF). As the data were skewed, the median and interquartile ranges were examined as measures of centrality and dispersion. Over 50 males and 50 females were recruited to each group. Due to gender differences the results are presented separately for males and females. Results: There was a significant increase in 18-ESF levels in four of the six groups $(\mathrm{p}<$ 0.05 ). As anticipated, both of the supervised toothbrushing groups experienced an increase in 18-ESF change in median from baseline to final for females 0.010 to $0.018 \mathrm{mg} / \mathrm{l}$, for males 0.014 to 0.017 $\mathrm{mg} / \mathrm{l}$, however the female dental health education group experienced an increase whilst the males did not and the converse was true of the control group where the male group experienced an increase in 18-ESF whilst the female group did not. Conclusion: 18 -ESF as a measure has some potential as an indicator of success in motivating children to brush more frequently, however further validation studies are required.

This research was funded by the Health Research Board, Ireland.

\section{6 \\ Remineralization of Early Caries Lesion Using Fluoride Varnish: A Methodology Study \\ I. Chedjieu*, A. Thusu, K. Ramalingam, B.T. Amaechi \\ manganmbondi@uthscsa.edu \\ University of Texas Health Science Center at San Antonio, Tex., USA}

Although the use of fluoride varnish to remineralize early caries lesion is widely practiced, there is no recommended method of application of the varnish for this purpose. The present study investigated the most appropriate method of varnish application for caries lesion remineralization. Thirty teeth were cut vertically and buccolingually to produce 60 tooth halves bearing buccal/ 
lingual surfaces. Another set of 30 teeth were cut vertically and mesiodistally to produce 60 halves bearing distal/mesial surfaces. Caries-like lesions were created on a defined spot on each surface by 7 days demineralization in acidified gel system made of $0.1 \mathrm{M}$ lactic acid ( $\mathrm{pH} 4.5)$ gelled with $6 \%(\mathrm{w} / \mathrm{v})$ hydroxyethyl cellulose. Tooth sections cut from each lesion were used to determine the baseline lesion depth (LD) using polarized light microscope (PLM). Samples with distal/mesial surfaces were arranged in pairs (30 pairs) with lesion surfaces facing each other to produce a contact point. Then samples were randomly assigned to three groups: (A) varnish applied directly on lesion surface, (B) varnish applied around the lesion, and (C) varnish applied on the contact point. Using an Artificial Mouth system, remineralization was conducted by $\mathrm{pH}$ cycling regimen consisting, each day, of three 1-hour acid challenge and artificial saliva treatment for the rest of the time. Following remineralization, tooth sections cut from each lesion were used to determine the post-treatment LD $(\mu \mathrm{m})$. Paired t test indicated significant difference $(p<0.001, \alpha=0.05)$ between the mean values of pre- and post-treatment LD in all groups. However, ANOVA and multiple comparisons showed no significant difference among the groups in percentage remineralization achieved: A $(29.5 \pm 16.5), \mathrm{B}(32.8 \pm 18.2), \mathrm{C}(28.5 \pm 13.3)$. The application of fluoride varnish around an early caries produced more remineralization than direct or contact point applications.

\section{7 \\ Modeling Fluoride Release from Varnish: Correlation of Coating Thickness and Surface Area}

P.J. Flanigan*, S. Kilmer, A.M. Pfarrer

pjflanigan@mmm.com

3M, St. Paul, Minn., USA

Fluoride varnishes are used to increase contact time between fluoride and enamel. Varnishes set on contact with saliva and release fluoride via wetting with saliva. Some clinicians apply varnish to specific sites while others apply varnish to the entire dentition. Previous research showed that a varnish that thins after application releases more fluoride than those that remain thick. The aim of this study was to model the relationship between coating thickness and surface area and fluoride release from $3 \mathrm{M}^{\mathrm{TM}} \mathrm{ESPE}^{\mathrm{TM}}$ Vanish $^{\mathrm{TM}} 5 \% \mathrm{NaF}$ White Varnish over $24 \mathrm{~h}$. A response surface regression was designed to evaluate thickness $(0.05-0.34 \mathrm{~mm})$ and area $\left(0.43-14.57 \mathrm{~cm}^{2}\right)$ of varnish coatings applied to dental composite material. The design included star points and contained 6 replicate center points. Each data point represents $n=10$ replicates. Varnish was applied with specific areas and thicknesses on slides and placed in $25 \mathrm{ml}$ of deionised water, $37^{\circ} \mathrm{C}$. After 1,4 , and $24 \mathrm{~h}$, water was collected and replaced with fresh water. The $\mathrm{F}$ concentration in the aqueous samples were determined using a calibrated fluoride ion selective electrode and F release calculated as $\mathrm{mg} F$ released/g of varnish applied. Using constant areas $\left(2.5\right.$ and $\left.12.5 \mathrm{~cm}^{2}\right)$; thin coatings $(0.1 \mathrm{~mm})$ released $29.2 \mathrm{mg} \mathrm{F} / \mathrm{g}(\mathrm{SD}=3.8)$ and $22.6 \mathrm{mg} \mathrm{F} / \mathrm{g}(\mathrm{SD}=$
9.9) respectively, while thick coatings $(0.3 \mathrm{~mm})$ released $21.2 \mathrm{mg}$ $\mathrm{F} / \mathrm{g}(\mathrm{SD}=1.6)$ and $21.1 \mathrm{mg} \mathrm{F} / \mathrm{g}(\mathrm{SD}=7.7)$ respectively over the $24 \mathrm{~h}$ period. A thick coating applied to a small area released a similar amount of fluoride as a thin coating applied to a large area. The model regression $\mathrm{R}-\mathrm{Sq}(\mathrm{adj})$ was $96.4 \%$. Thin coatings of varnish exhibit greater fluoride release into external liquid medium than thick coatings.

Research was funded by $3 \mathrm{M}$ ESPE.

\section{8 \\ Salivary Fluoride Retention after Application of Home Use Topical Fluorides \\ T.T. Tauböck*, T. Attin, B. Sener, W. Buchalla \\ tobias.tauboeck@zzm.uzh.ch \\ Department of Preventive Dentistry, Periodontology and \\ Cariology, University of Zurich, Switzerland}

The aim of this in vivo study was to evaluate salivary fluoride retention after treatment with topical fluoride agents intended for home use. Ten volunteers used Elmex mouthrinse $(10 \mathrm{ml}, 250$ ppm F), Elmex toothpaste (1 g, 1,400 ppm F) and Elmex Gelée $(1 \mathrm{~g}, 12,500 \mathrm{ppm} \mathrm{F})$ for $2 \mathrm{~min}$, respectively, or received no fluoride treatment (control). Samples of unstimulated whole saliva were collected 0 (slurry), 10, 30, 60 and 240 min after each treatment. Fluoride contents of saliva samples were measured by means of a fluoride ion-specific electrode. Salivary slurry contained $163 \pm$ 41, $169 \pm 58$ and 1,440 \pm 497 ppm F directly after application of Elmex mouthrinse, Elmex toothpaste and Elmex Gelée, respectively. Fluoride content in the non-fluoride control was close to or below the lower limit of quantification $(0.08 \mathrm{ppm}$ F). Fluoride concentration in saliva decreased with time in all fluoride groups. The mouthrinse, the toothpaste and the gel resulted in significantly higher mean salivary fluoride concentrations compared to the non-fluoride control up to $240 \mathrm{~min}$. At each time point, the gel resulted in the significantly highest salivary fluoride concentration of all groups. No significant differences in salivary fluoride concentrations were observed between the mouthrinse and the toothpaste, independent of the time point of measurement. Volunteers with low initial unstimulated saliva flow rates showed a tendency towards higher salivary fluoride content. It is concluded that all fluoride measures tested led to elevated salivary fluoride concentrations at least up to 240 min with the highest concentrated product (gel) leading to the highest salivary fluoride concentration. 


\section{9}

\section{Optimal Ratio of Calcium-Phosphate lons for the Remineralization by Phosphoryl Oligosaccharides of Calcium with Fluoride}

T. Tanaka*, T. Kobayashi, H. Takii, H. Kamasaka, T. Kuriki

tanaka-tomoko-2@glico.co.jp

Institute of Health Sciences, Ezaki Glico Co., Ltd., Osaka, Japan

Phosphoryl oligosaccharides of calcium (POs-Ca ${ }^{\circledR}$ ) containing $5 \%(\mathrm{w} / \mathrm{w})$ calcium provides soluble calcium in saliva by stabilizing calcium ions with its phosphoryl residue in maltooligosaccharides, and effectively enhances the remineralization and recrystallization of enamel lesions. Fluoride ions with POs-Ca were possibly effective for the early caries lesion. The aim of this study was to investigate the enhancement of enamel remineralization with POs-Ca and fluoride and the optimal calcium-phosphate $(\mathrm{Ca} / \mathrm{P})$ molar ratio for the remineralization with POs-Ca under the existence of fluoride of enamel lesions in vitro. Bovine tooth blocks were demineralized using the two-layer demineralization method by ten Cate et al. To determine the optimal concentration of fluoride for the remineralization with POs-Ca, demineralized enamel blocks were treated with the mineral solutions containing $1.0,10$, and $100 \mathrm{ppm}$ fluoride ions with $0.36 \%$ POs-Ca and $3.6 \mathrm{~mm}$ phosphate ions. Then, the other demineralized enamel blocks were treated with five different concentrations of POs-Ca (ratio of calcium-phosphate $(\mathrm{Ca} / \mathrm{P})$ were $0.4-3.0)$ under the existence of 3.6- $\mathrm{mm}$ phosphate and fluoride ions at the optimal concentration ( $\mathrm{pH}$ 6.5). These enamel blocks were incubated for $24 \mathrm{~h}$ at $37^{\circ} \mathrm{C}$. Then, they were cut into sections and subjected to transversal microradiography. Only 1-ppm fluoride ion did not inhibit the remineralization with POs-Ca. The recovery rate was significantly reduced by excess calcium (more than $10 \mathrm{~mm}$ ) or excess fluoride ions $(100 \mathrm{ppm})(\mathrm{p}<0.05$, Scheffe's test). The highest recovery rate of remineralization ( $\mathrm{p}<0.05$, Scheffe's test), $26.7 \pm 2.3 \%(\mathrm{n}=5)$ was observed at $\mathrm{Ca} / \mathrm{P}$ ratio of 1.67 with 1.0 -ppm fluoride ions, which is higher than the recovery rate for the remineralization with 1-ppm fluoride without POs-Ca (10.1 $\pm 2.8 \%)$. Hence, it was concluded that POs-Ca provides soluble calcium even with 1-ppm fluoride, and enhances the mineralization of early caries lesion at $\mathrm{Ca} / \mathrm{P}=1.67$.

Ezaki Glico Co., Ltd.

\section{0 \\ Enamel Remineralization Effectiveness of Different Fluoride-Containing Mouthrinses in Conjunction with Toothpaste}

\author{
D. Sharma ${ }^{\mathrm{a},}{ }^{*}$, D. Ricci-Nittel $^{\mathrm{a}}$, B. Mutti $^{\mathrm{a}}$, R.L. Karlinsey ${ }^{\mathrm{b}}$, \\ A.C. Mackey ${ }^{b}$ \\ bmutti@its.jnj.com \\ aJohnson \& Johnson CPPWW, Morris Plains, N.J., and \\ b Indiana Nanotech, Indianapolis, Ind., USA
}

The aim of this work was to compare the ability of fluoridated Essential-Oil mouthrinse (LISTERINE ${ }^{\circledR}$ Total Care (LTC)) and neutral $\mathrm{pH}$ fluoridated mouthrinse $\mathrm{ACT}^{\circledR}$ Total Care (ATC) in conjunction with Crest ${ }^{\circledR}$ Cavity Protection, a fluoridated Toothpaste (FTP), to promote remineralization into demineralized dental enamel versus a negative control. This observer blinded, controlled $\mathrm{pH}$ cycling study employed a randomized parallel group design testing the following groups: (A) Crest ${ }^{\circledR}$ FTP + distilled (DI) water, (B) Crest ${ }^{\circledR}$ FTP +LTC and (C) Crest ${ }^{\circledR}$ FTP +ATC. Human enamel specimens ( $n=10$ /group) with caries-like incipient lesions were balanced according to baseline surface micro hardness (SMH) values (25-45 Vickers hardness Number (VHN)). Specimens were exposed to $\mathrm{pH}$ cycling model for 20 days and SMH was measured after 5, 10 and 20 days. Each day specimens were subjected to four one-minute treatments (treatments 1 and 3 were with Crest ${ }^{\circledR}$ FTP whereas 2 and 4 were either test mouthrinse or water), one four-hour acid challenge (carbopol-lactic acid solution, $\mathrm{pH} 4.8$ ), and one hour artificial saliva ( $\mathrm{pH}$ 7.0) in between these treatments. After the 20 days of $\mathrm{pH}$ cycling all specimens underwent a simulated plaque acid challenge (SPAC) using carbopol-lactic acid solution ( $\mathrm{pH} 4$.8) for one 2-hour period. After 20 days of cycling treatments, mean \pm SEM changes in SMH were: (A) $71.10 \pm 8.89^{\mathrm{a}}$, (B) $107.18 \pm 18.21^{\mathrm{b}}$, (C) $67.11 \pm 9.50^{\mathrm{a}}$, where $\mathrm{a}<\mathrm{b}($ ANOVA, SNK, $\mathrm{p}<0.05)$ and after SPAC were $(\mathrm{A})$ $57.64 \pm 7.53^{\mathrm{a}}$, (B) $78.16 \pm 14.50^{\mathrm{b}}$ and (C) $64.54 \pm 7.60^{\mathrm{a}}$, b , where $\mathrm{a}<\mathrm{b}$ (ANOVA, SNK, $\mathrm{p}<0.05$ ). Based on significance of these results and remineralization observed 5 and 10 days of $\mathrm{pH}$ cycling we conclude that LISTERINE ${ }^{\circledR}$ Total Care ${ }^{\mathrm{TM}}$ is not only effective but provides significantly enhanced enamel remineralization benefits compared to fluoride from toothpaste alone and toothpaste plus ACT ${ }^{\circledR}$ Total Care ${ }^{\mathrm{TM}}$.

This work was supported by Johnson and Johnson Consumer and Personal Products Worldwide. 


\section{Caries Research}

\section{Session 2 Clinical Studies}

\section{1 \\ Preventive Effect of Two Different Pit and Fissure Sealant Materials: 24-Month Follow-Up \\ S. Prentic Bakic a , H. Juric ${ }^{\mathrm{b}, *}$ \\ juric@sfzg.hr \\ aPrivate practice 'Dr. Marina Jezina', Split, and 'SSchool of Dental Medicine, Department of Pediatric and Preventive Dentistry, University of Zagreb, Croatia}

Occlusal caries of permanent molars is a problem in every day practice because that tooth surface is still most frequently affected by caries. The purpose of this study was to evaluate the effect of two different fissure sealants on prevention of occlusal caries in permanent molars. This study was conducted on two examined and one control group. In first group, 392 teeth in 98 patients, aged 7-9 years, were sealed with resin based material (Helioseal F). In second group, 260 teeth in 65 patients, same age, were sealed with glass-ionomer cement (Fuji Triage). In control group, 300 teeth were examined in 75 patients of the same age, whose permanent molars were never sealed. Control examinations of sealed teeth were conducted after 6,12 and 24 months. Retention of the sealants was determined as well as occurrence of caries on occlusal surfaces. For caries scoring the ICDAS II criteria were used. Results were statistically analyzed using t-test and $\chi^{2}$ test. At the end of the study, resin based sealant showed much better retention with complete retention in $72 \%$ fully retained, $22 \%$ partially lost and $6 \%$ totally lost while for the glass-ionomer cement $19 \%$ were fully retained, $36 \%$ partially lost and $45 \%$ totally lost $(\mathrm{p}<0.05)$. The occurrence of new occlusal caries lesions at the dentinal level ( $\geq$ code 4 ) at the end of study was $25 \%$ in resin based sealant group and $30 \%$ in the glass ionomer group ( $p>0.05)$. In the control group, $65 \%$ of occlusal fissures had primary caries lesion ( $\geq$ code 4 ) or had been restored $(p<0.05)$. We can conclude that pit and fissure sealing is a valuable method in preventing occlusal caries and the tested materials could be recommended for everyday use.

\author{
22 \\ Placebo-Controlled Randomized Clinical Trial on \\ Proximal Caries Infiltration: 3-Year Follow-Up \\ H. Meyer-Lueckel ${ }^{\mathrm{a}, *}$, K. Bitter $^{\mathrm{b}}$, S. Paris ${ }^{\mathrm{a}}$ \\ meyer-lueckel@konspar.uni-kiel.de \\ ${ }^{a}$ Clinic for Operative Dentistry and Periodontology, School \\ of Dental Medicine, Christian-Albrechts-Universität zu Kiel,

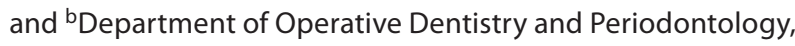 \\ Charité - Universitätsmedizin Berlin, Germany
}

Caries infiltration is a micro-invasive therapeutic approach to arrest progression of caries lesions. The aim of this randomized split-mouth placebo-controlled clinical trial was to assess, whether resin infiltration of proximal lesions in combination with selfapplied non-invasive measures is more efficacious than non-invasive measures alone with respect to inhibition of caries progression. In 22 young adults 29 pairs of proximal caries lesions radiologically extending into inner half of enamel (E2) or outer third of dentin (D1) were randomly allocated to either one of two treatments. In the test group, lesions were infiltrated (ICON, preproduct; DMG). A placebo treatment was performed in the control group. All subjects received instructions for non-cariogenic diet, flossing and fluoride use. Outcome was radiographic lesion progression as evaluated by two blinded examiners separately by pairwise comparison (PW) or pairwise comparison + digital subtraction radiography (PW+DSR). Subsequently, a consensus was obtained. After 36 months, 24 lesion pairs (19 patients) could be re-evaluated radiographically using standardized bitewing holders (pre-product; DMG). Two lesion pairs were excluded due to invasive treatment by other dentists, two patients (3 lesion pairs) were not eligible for the follow-up. No unwanted effects could be observed. Intra- and inter-rater reliability yielded moderate to substantial agreement. The consensus results for PW+DSR revealed progression of $1 / 24(5 \%)$ and $11 / 24(46 \%)$ lesions in the test and in the control group, respectively ( $\mathrm{p}=0.002$; McNemar test) (PW: test: $1 / 24$, control: $9 / 24 ; \mathrm{p}=0.008)$. For proximal caries lesions extending around the enamel-dentin junction (E2, D1) res- 
in infiltration in combination with self-applied non-invasive measures is more efficacious in reducing lesion progression compared with self-applied non-invasive measures alone.

The study was supported by DMG, Hamburg and conducted at Charité-Universitätsmedizin Berlin, which is hereby acknowledged. H.M.L. and S.P. receive royalties from DMG related to the caries infiltration technique.

\section{3}

\section{Oral Health Related to Stages of Change}

A. Verhoef*, C.O.V.M. Gresnigt-Bekker, C. van Loveren

a.verhoef@acta.nl

Academic Centre for Dentistry (ACTA), Amsterdam,

The Netherlands

Motivational interviewing relies on the stage of change of a patient: (1) no intention to change, unaware of the need to, (2) not ready to change, although aware of the need to, (3) ready to change within a month, (4) has changed behavior within the past 6 months, (5) has changed behavior for at least 6 months. Aim of this study was to relate the stage in which patients indicate to be to their oral health status. Patients $(n=158)$ classified themselves into stages of change by following a flowchart. Current periodontal health by DPSI (Dutch Periodontal Screening Index), DMFS and caries risk (based on sugary intakes, DMFS and active caries lesions) were extracted from their patient records. 127 patients (65\% male, $35 \%$ female; mean age: $42.5 \pm 14.9$ years) filled in the flowchart correctly. They divided into stage 1 to 5 by 28.3, 21.3, $15.7,11.8$, and $22.8 \%$, respectively. Mean DMFS for the stages: 20.1 $\pm 22.6,35.1 \pm 29.5,8.75 \pm 9.0,37.4 \pm 32.0,30.3 \pm 27.9$, respectively. Patients in stage 3 had lower DMFS than patients in stage 2,4 and 5 (Tukey B, p < 0.05). DPSI in stage 1 to 5 was $1.7 \pm 0.7$, $2.1 \pm 0.6,1.5 \pm 0.6,1.7 \pm 0.7,1.9 \pm 0.8$. Patients in stage 3 had a lower DPSI than patients in stage 2 (Tukey B, p < 0.05). No differences between the stages were found in caries risk with an overall distribution of $29 \%$ low risk, $59 \%$ middle risk, and $11 \%$ high risk. In conclusion patients classified themselves into stages of change towards adopting new oral hygiene measures and those in stage 3 had a better oral health than patients in stage 2, 4 and 5 .

\section{4 \\ Evaluation of a Preventive Program Aiming at Children with Increased Caries Risk Using ICDAS II Criteria \\ K. Pieper ${ }^{\mathrm{a},}{ }^{*}$, K. Weber $^{\mathrm{a}}$, S. Stein ${ }^{\mathrm{b}}$, M. Heinzel-Gutenbrunner ${ }^{\mathrm{a}}$, A. Jablonski-Momeni ${ }^{\mathrm{a}}$, J. Margraf-Stiksrud ${ }^{\mathrm{b}}$ \\ pieper@med.uni-marburg.de \\ ${ }^{\mathrm{a}}$ Department of Pediatric and Community Dentistry and \\ ${ }^{b}$ Faculty of Psychology, Philipps University Marburg, Germany}

A selective intensified prevention (SIP) was introduced in Marburg County in 1995. SIP is offered at individual schools in underprivileged districts including enhanced health education and oral hygiene instructions as well as fluoride varnish applications four times per year. The effect of the program was evaluated in sixth graders (mean age: 12.06 ) in comparison to a control region. Caries experience was recorded using the ICDAS II. Teeth were dried with compressed air prior to examination and CPI probes were used for assessing ICDAS scores 5 and 6. The statistical evaluation was performed by means of the software package SPSS 17.0. To compare the mean caries scores of various subgroups, non parametric tests were performed. In Marburg 236 children were examined, 689 were participating in the control region. The samples were parallelized by constructing matched pairs with children from the control region in order to avoid bias due to differences in the proportion of ethnicities. Matching criteria were age, gender, ethnicity and maternal education. Especially ICDAS codes 3 to 6 were found more frequently in children from the control region compared to those from Marburg County. Combining ICDAS scores 3 to 6 children from the control region (mean $\mathrm{D}_{3-6} \mathrm{MFT}$ : 1.73) showed roughly double the caries experience compared to the test group (mean $\mathrm{D}_{3-6} \mathrm{MFT}$ : 0.88 , p < 0.005). The $\mathrm{D}_{5,6} \mathrm{MFT}$ score of the test group was 0.50 , the corresponding value of the reference group amounted to 0.77 ( $\mathrm{p}=$ 0.043 ). Differences between groups may be detected earlier and with less numbers of cases, by using a combination of ICDAS scores 3 to 6 compared to WHO standard.

German Federal Government (Grant No. BMBF 01EL0617).

\section{5 \\ A New Method for Estimating Survival of Sealant Retention \\ J.E. Frencken ${ }^{\mathrm{a}, *}$, X. Chen ${ }^{\mathrm{b}}$, M.Q. Du ${ }^{\mathrm{b}}$, M.W. Fan ${ }^{\mathrm{b}}$, J. Mulder ${ }^{\mathrm{a}}$, M.C.D.N.J.M. Huysmans ${ }^{\mathrm{a}}$ \\ j.frencken@dent.umcn.nl \\ ${ }^{a}$ Radboud University Medical Centre Nijmegen, Nijmegen, \\ The Netherlands; bWuhan University, Wuhan, China}

Traditionally, survival of sealant retention in occlusal surfaces is estimated by dichotomizing fully and partially retained versus completely lost sealants based on the total surface as the unit of evaluation. However, the group of partially retained sealants range from those in which pits and fissures are almost completely covered with sealant material to those in which pits and fissures are almost completely re-exposed to the oral environment. This wide range of states of partially retained sealants makes the outcome of a survival analysis on sealant retention unrealistic. A new method of determining the retention of sealants is proposed by evaluating its state for each of the 3 sections (mesial-central-distal) in which the occlusal surface is divided. According to the modified categorization, a sealant is considered partially retained if the material is fully present in two sections and partially present or completely lost in the remaining section. The traditional and modified categorization of partially retained sealants were applied to estimate the survival of fully and partially retained resincomposite (Clinpro) and high-viscosity glass-ionomer (Ketac Molar Easymix) sealants after 2 years using the Kaplan-Meier method. The Jackknife method was applied for calculating standard errors (SE). Using the traditional categorization, the 2-years 
survival and SE of fully and partially retained resin-composite sealants and high-viscosity glass-ionomer was $86.2 \pm 2.3$ and $78.1 \pm 2.8$, respectively whereas the survival outcomes were 55.7 \pm 3.1 and $44.6 \pm 3.4$, respectively using the modified categorization. The difference in survival between the two ways of calculating partially retained sealants was statistically and clinically significant. In conclusion, the modified categorization in analyzing sealants' retention provides a more realistic method of sealants' ability to prevent dental caries.

Ministry of Science and Technology, China, and Royal Netherlands Academy of Science.

\section{6 \\ Bacterial and Behavioral Factors Associated with Early Childhood Caries in Children Aged 9-18 Months \\ N. Shakavets*, T. Tserakhava \\ n.shakavets@gmail.com \\ Department of Pediatric Dentistry, Belarusian State Medical \\ University, Belarus}

The aim of this study was to investigate the relationship between demographic and behavioral factors potentially associated with early childhood caries and to assess the relationships of salivary levels of mutans streptococci (MS) in mothers and their 9- to 18-month-old children. 79 mother-and-child pairs were examined and MS salivary counts (CRT/Ivoclar Vivadent) were obtained from children and mothers. Early childhood caries was diagnosed based on the presence of any carious lesion on any primary tooth surface using $\mathrm{d}_{1-4} \mathrm{mft}$ and $\mathrm{d}_{1-4} \mathrm{mfs}$ index $\left(\mathrm{d}_{1}-\right.$ noncavitated lesion, $d_{2}-$ caries limited to enamel, $d_{3}-$ caries in dentin, $\mathrm{d}_{4}-$ caries involving pulp). The interview of mothers was based on a questionnaire designed to collect demographic data and information on the education of the parents, feeding practices, oral health and hygiene. $84.8 \%$ of the infants were caries free. The $\mathrm{d}_{1-4} \mathrm{mft}$ index was $0.58 \pm 0.17, \mathrm{~d}_{1-4} \mathrm{mfs}$ index was $1.2 \pm$ 0.43 . Visible plaque was detected on the upper incisors of $52 \%$ children and gingivitis was recorded in $15.2 \% .96 \%$ of mothers and $28 \%$ of infants were harbouring MS. The proportion of MS positive and dmft-positive children were the highest in group of mothers with the level of MS $\geq 10^{6}(r=0.43)$. There was strong correlation between presence of salivary MS in infants and dental caries incidence $(r=0.7)$. Those mothers who had university degrees brushed their infants' teeth more often and fed their children with sweets less rarely than the other ones. Presence of MS in children, night feeding practice and daily sugar consumption were significantly associated with the presence of caries $(p<0.05)$. It was concluded that not only bacterial but behavioral factors are closely associated with caries in very young children.

Ivoclar Vivadent/Liechtenstein.

\section{7 \\ Effect of Resin Infiltration Material on White Spot Lesions after Brackets Removal}

M.M.A. Mohsen ${ }^{\mathrm{a}, *}$, S.M. Hammad ${ }^{\mathrm{b}}$, I.M.G. El Zayat ${ }^{\mathrm{a}}$, M. El Banna

salah_mai@yahoo.com

aDepartment of Operative Dentistry, Misr International

University, Cairo, and ' ${ }^{b}$ Department of Orthodontics, Mansoura

University, Cairo, Egypt

The aim of this investigation was to evaluate the effect of resin infiltration (ICON) on white spot lesions (WSLs) after debonding fixed orthodontic brackets in vivo. A total number of 18 patients ranged from 14 to 18 years old who agreed to participate in the study were recruited among patients treated with fixed orthodontic appliances at the Orthodontic Clinic, Mansoura University. For every patient, the maxillary six anterior teeth were isolated with cotton rolls and air-dried for $5 \mathrm{~s}$. Patients were divided into 2 groups of 9 patients each; by a visual score based on the extent of demineralization, according to the classification of the WSLs proposed by Tufekci et al. [Angle Orthod 2011;81:206-210]. Group 1: WSLs were visible lesions without surface disruption (mild demineralization). Group 2: WSLs showed a roughened surface but not requiring a restoration (moderate demineralization). Standardized digital photographs were taken before and after Icon application, 1 week after debonding. Image analyzing soft ware was used to evaluate the change in color of the WSLs before and after the application of ICON. Statistical analysis was done with Wilcoxon's signed rank test. The results revealed $80 \%$ masking of the white lesion in group 1 and 65\% in group 2. Also, analysis of the colour changes in both groups 1 and 2 in the scale ranged from 0 to 255 and showed a shift from 155.1 ( \pm 46$)$ for the pre-photographs 203.4 ( \pm 33.3$)$ for the post-photographs $(\mathrm{p}=0.038)$, indicating a significant shift towards white color. Resin infiltration showed superior results in the limitation of the WSLs, so it is recommended to be applied one week after debonding of fixed orthodontic appliances.

Misr International University.

\section{8 \\ Sealing Lesions of Occlusal Caries: A Randomized Clinical Trial \\ F.C.M. de Santana Giongo a , B. Mua a , B.B. e Silva ${ }^{\mathrm{a}}$, V. Qvist ${ }^{\mathrm{b}}$, M. Maltz ${ }^{\mathrm{a}, *}$ \\ marisa.maltz@gmail.com \\ a Department of Social and Preventive Dentistry, Faculty of \\ Dentistry, Federal University of Rio Grande do Sul, Brazil; \\ ${ }^{b}$ Department of Cariology and Endodontics, School of \\ Dentistry, University of Copenhagen, Denmark}

The aim of this randomized controlled clinical trial was to compare the efficacy of sealing of carious lesions and restorative treatment of occlusal lesions in permanent teeth. The sample consisted of 52 occlusal lesions from 47 patients. The maximum depth of the lesions was halfway through the dentine assessed by 
bitewing radiograph. The inclusion criteria were presence of cavity (lesion in which biofilm control was not possible) and radiolucency limited to the middle third of dentin. The teeth were assigned to sealant treatment $(n=26)$ or restorative treatment $(n=$ 26). Clinical and radiographic examinations were performed after 12 months. Outcomes were clinical performance of sealant and restoration and the prevalence of regression, inactivation and progression of lesion and presence of tertiary dentine by radiographic examinations. The data were submitted to Fisher's exact test. After one year, 49 lesions were available for examination. The success rates in sealant and restoration groups were 95.8 and $100 \%$, respectively ( $p>0.05$ ). There was one total loss in the sealant group. No teeth showed caries progression; regression was observed in one case (sealant group) and tertiary dentin was founded in 5 lesions in the sealant group and 1 lesion in the restoration group $(p>0.05)$. In conclusion sealants can be used as a therapeutic treatment for cavitated lesions since they prevent the progression of carious lesions and preserving tooth structure when compared to restorative treatment.

Supported by Brazilian Ministry of Education through its Agency for Support and Evaluation of Graduate Education (CAPES) and Federal University of Rio Grande do Sul (UFRGS).

\section{9 \\ Determination of the Prevalence of Molar-Incisor Hypomineralisation Using Different Index Teeth and Distribution Patterns}

\author{
D. Mach ${ }^{\mathrm{a}, *}$, E. Thiering ${ }^{\mathrm{b}}$, J. Heinrich ${ }^{\mathrm{b}}$, R. Hickela ${ }^{\mathrm{a}}$, J. Kühnisch \\ dmach@dent.med.uni-muenchen.de \\ Department of Conservative Dentistry and Periodontology, \\ Maximilians University of Munich, Germany
}

This epidemiological study aimed to analyse the distribution of enamel hypoplasia $(\mathrm{EH})$ and its influence on the determination of the prevalence of molar-incisor hypomineralisation (MIH). The phenomenon of MIH is diagnosed if EHs are present on at least one first permanent molar (PFM). Frequently the permanent incisors are also affected. Unfortunately, only limited information about the pattern of distribution of $\mathrm{EH} / \mathrm{MIH}$ is available. Therefore, 465 children (10.2 years) of the 10-year follow-up of the LISAplus cohort study were examined in Munich, Germany. The LISAplus study is an ongoing birth cohort study of unselected infants, designed to assess 'influences of lifestyle-related factors on the immune system and the development of allergies in childhood'. Demarcated opacities, enamel disintegrations and atypical restorations were registered on all permanent teeth (HT) and surfaces (HS). Each child was categorised according to the following groups: (1) children with $\mathrm{EH}$ on at least one PFM (1-4 PFM and 0-8 incisors), (2) children with EH on PFM and permanent incisors (1-4 PFM and 1-8 incisors), and (3) children with $\mathrm{EH}$ on other permanent teeth (incisors, canines, premolars). The mean number of EHs was $0.6( \pm 1.3)$ on HT and 0.7 $( \pm 1.8)$ on HS among the whole study population. $12.0 \%$ of all children were classified to group $1(3.2( \pm 1.8) \mathrm{HT} ; 4.3( \pm 3.3) \mathrm{HS})$, $8.0 \%$ to group $2(4.0( \pm 1.6) \mathrm{HT} ; 5.5( \pm 3.3) \mathrm{HS})$ and $12.3 \%$ to group $3(1.5( \pm 0.8)$ HT; 1.5 ( \pm 0.9$)$ HS). Demarcated opacities were recorded in $86-98 \% / 80-98 \%$ of all hypomineralised teeth/ surfaces. On the basis of our study it can be concluded that different distribution patterns are linked with different prevalence rates. $\mathrm{EH}$ should be recorded on all primary and permanent teeth/surfaces to gain more information about the important question which threshold(s) should be applied to identify subjects with EH/MIH.

This study was funded by the German Research Foundation (Deutsche Forschungsgemeinschaft, FKZ KU 2518/1-1 and HE 3294/7-1) and GABA GmbH, Lörrach, Germany.

\section{0 \\ Oral Manifestations of Autoimmune Polyendocrine Syndrome Type I \\ M. Marthinussen-Cuida ${ }^{\mathrm{a}, *}$, M.M. Erichsen ${ }^{\mathrm{e}}$, M.V. Jonsson ${ }^{\mathrm{b}, \mathrm{f}}$, A.-C. Altenau', E.S. Husebye ${ }^{\mathrm{d}}$ \\ Mihaela.Cuida@iko.uib.no aDepartment of Clinical Dentistry - Cariology, ${ }^{\mathrm{b}}$ Dental University Hospital - Oral Radiology, ' ${ }^{\mathrm{D}}$ Dental University Hospital - Cariology, Institute of Medicine - Section for Endocrinology, Faculty of Medicine and Dentistry, University of Bergen, e'Department of Medicine, Haukeland University Hospital, 'Institute of Medicine - Section for Rheumatology, University of Bergen, Norway}

Autoimmune polyendocrine syndrome type I (APS-I) is a rare autosomal-recessive disease caused by mutations in the autoimmune regulator (AIRE) gene. The prevalence of APS-I in Norway was estimated to be about 1:90,000. Besides the three main components chronic mucocutaneous candidiasis, hypoparathyroidism, and adrenal insufficiency, many patients display ectodermal manifestations, among them enamel dystrophy. There is also increased mortality in squamous carcinoma of the oral cavity, probably secondary to chronic candidiasis. The literature is especially sparse regarding information on oral manifestations in APS-I patients. Therefore our goal was to define the oral clinical picture and the oral health condition in these patients. Seven patients with APS-I were studied, 2 males and $5 \mathrm{fe-}$ males, median age of 45 years (24-60). The patients had a detailed oral examination and radiographic evaluation. In addition saliva flow measurements and oral candidiasis test were performed. Oral findings included oral candidiasis, angular cheilitis, enamel hypoplasia and reduced unstimulated salivary secretion. Of 7 patients, 5 had oral candidiasis, 3 angular cheilitis and 3 had pathological unstimulated salivary flow $(<0.1 \mathrm{ml} / \mathrm{min})$. Enamel hypoplasia in the form of horizontal hypoplastic bands or generally pitted enamel was seen in 6 patients. One patient presented impacted wisdom teeth with defective mineralization. In conclusion patients with APS-I frequently present oral manifestations and therefore need lifelong follow-up for the detection of new components of the disease. Careful control of oral candidiasis is mandatory for early detection of carcinoma. Early recognition and appropriate management of enamel defects and xerostomia may prevent dental disease, such as dental caries and help to improve the quality of life. 
The Norwegian Directorate of Health Dental University Hospital "C Faculty of Medicine and Dentistry, University of Bergen, Bergen, Norway.

\section{1 \\ Use of a Local Sorbitol-Containing Chewing Gum Product to Prevent Dental Caries in Saudi Arabia}

K.S. Al-Khalifa, ${ }^{\text {* }}$, E.O. AlShwaimi ${ }^{\text {b }}$

kalkhalifa@ud.edu.sa

aDivision of Dental Public Health, Department of Preventive

Dental Sciences, and bivision of Endodontics, Department of

Restorative Dental Sciences, College of Dentistry, University of

Dammam, Saudi Arabia

The chewing of sorbitol-containing gum has been suggested in the dental literature to reduce caries rates. The purpose of this study was to assess the effectiveness of sorbitol-containing chewing gum in reducing the incidence of caries in caries-active adolescents. Participants were 150 adolescents with current or recent caries lesions from three different schools in Dammam, Saudi Arabia. The first group received sorbitol-containing chewing gum (Nova from Batook Chewing Gum Industries Ltd., Saudi Arabia) four times a day. The second group received sorbitol-containing chewing gum two times a day. While the third group acted as a control group. Demographic information collected was: age, gender, socioeconomic status and education level of the parents. For all three groups a complete dental exam was conducted at baseline and at the end of the trial period (three months). A caries risk assessment survey was distributed that included a dietary analysis. Bacterial tests for cariogenic bacteria Streptococcus mutans and Lactobacilli was conducted using the Caries Risk Test (CRT) by Vivadent/Ivoclar, where counts $\geq 10^{5} \mathrm{CFU} / \mathrm{ml}$ saliva were considered 'high' bacterial count. Saliva was analyzed for its acidity (using $\mathrm{pH}$ strips), as well as flow and type (sticky vs. watery). All three groups were similar in their characteristics at baseline in terms of oral health and demographic background. At the end of the trial, there was no significant difference $(p>0.05)$ in the 'high' bacterial count between the two groups that used sorbitol-containing chewing gum and the control group. The difference in the 'high' bacterial count between baseline and end of trial was as follows: S. mutans $10 \%$ and Lactobacilli $8 \%$ for the first group (4 gum sticks/day), S. mutans 12\% and Lactobacilli $8 \%$ for the second group (2 gum sticks/day) and for the third group (control), S. mutans was $8 \%$ and Lactobacilli was $6 \%$. There was no statistical difference in the bacterial count between the two groups that used sorbitol-containing chewing gum ( $p>0.05)$. The use of sorbitol-containing chewing gum twice a day represents a potentially effective caries prevention method, especially with those patients who are mostly at risk.

Deanship for Scientific Research, University of Dammam, Saudi Arabia.

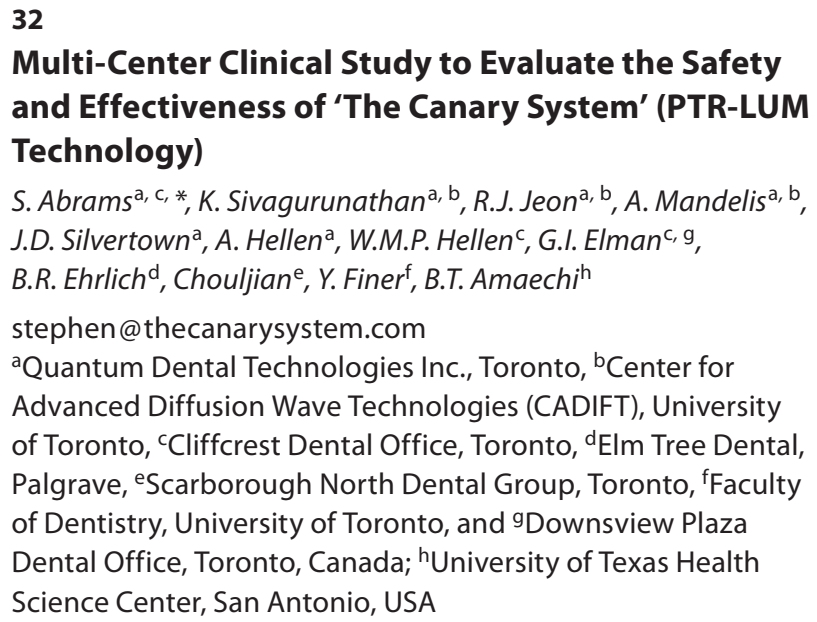
and Effectiveness of 'The Canary System' (PTR-LUM Technology)

S. Abrams ${ }^{\mathrm{a}, \mathrm{c}, *}$, K. Sivagurunathan ${ }^{\mathrm{a}, \mathrm{b}}$, R.J. Jeon ${ }^{\mathrm{a}, \mathrm{b}}$, A. Mandelis $^{\mathrm{a}, \mathrm{b}}$, J.D. Silvertown ${ }^{\mathrm{a}}$, A. Hellen ${ }^{\mathrm{a}}$, W.M.P. Hellen ${ }^{\mathrm{c}}$, G.I. Elman ${ }^{\mathrm{c}, \mathrm{g}}$, B.R. Ehrlich ${ }^{\mathrm{d}}$, Chouljian ${ }^{\mathrm{e}}$, Y. Finer ${ }^{\mathrm{f}}$, B.T. Amaechi ${ }^{\mathrm{h}}$

stephen@thecanarysystem.com

${ }^{a}$ Quantum Dental Technologies Inc., Toronto, ${ }^{b}$ Center for Advanced Diffusion Wave Technologies (CADIFT), University of Toronto, ${ }^{\mathrm{C} C l i f f c r e s t}$ Dental Office, Toronto, ${ }^{\mathrm{d}}$ Elm Tree Dental,

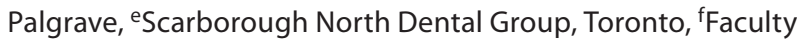
of Dentistry, University of Toronto, and 9Downsview Plaza Dental Office, Toronto, Canada; ' ${ }^{-}$University of Texas Health Science Center, San Antonio, USA

Using frequency domain photothermal radiometry (PTR) and modulated luminescence (LUM), Quantum Dental Technologies (QDT) has developed and tested a portable PTR-LUM-based instrument, The Canary System (CS), for the detection and monitoring of dental caries. The specific aims of this multi-center study were to (1) evaluate the safety and efficacy of this device in a wide range of clinical scenarios seen in dental practice, (2) develop a treatment scale that relates to caries lesion severity, and (3) establish the ability of this scale to detect and monitor changes in caries and erosion lesion severity over time in response to various remineralization therapies. This clinical study, approved by Health Canada, was conducted on 80 patients from four dental clinics. PTR-LUM responses were measured over 1,000 regions on various tooth surfaces. The CS responded to different clinical conditions by generating a Canary Number $(\mathrm{CN})$, an algorithm based upon the PTR-LUM reading from the tooth surface. The CNs were recorded from healthy surfaces, white/brown spots, cavitated/non-cavitated lesions and demineralized/remineralized surfaces. All caries lesions were ranked using modified ICDAS II criteria scoring only non-cavitated lesions. Neither adverse events nor soft/hard tissue trauma were observed. The $\mathrm{CN}$ varied with the severity, type, and nature of the lesion. The CS was able to use the CNs to indicate change in lesion activity over time after exposure to different remineralization agents. Pearson's correlation coefficient showed a good correlation $\left(r^{2}=0.7\right)$ between $\mathrm{CN}$ and ICDAS II values. In conclusion, the Canary System is safe, and was able to use the Canary Numbers to discriminate between healthy and carious tooth surfaces as well as indicate varying severity of caries lesion.

Quantum Dental Technologies. 


\section{3 \\ Effect of Chewing-Gum Containing POs-Ca and Fluoride on Oral Health Status in School Children}

T. Doi*, T. Miyake, M. Uene, K. Jin, K. Kawasaki, M. Kambara

doi@cc.osaka-dent.ac.jp

Department of Preventive and Community Dentistry,

Osaka Dental University, Japan

The purpose of this clinical study was to evaluate the effect of POs-Ca and fluoride combination chewing-gum intake to the oral health status in elementary school children. The caries status of 69 subjects ( 6 or 7 years of age) was determined by visual inspection. The salivary levels of mutans streptococci (Dentocult ${ }^{\circledR}$. $\mathrm{SM}$ ), saliva buffer capacity (Dentobuff ${ }^{\circledR}$-strip) were determined and the occlusal surface of the 1st permanent molar was examined using QLF. The activity of early caries lesions was classified as progressing, arrested, or recovering by QLF examination. These examinations were repeated by 6 -month intervals for one year. Chewing-gum containing POs-Ca was given 47 subjects (POs-Ca group), and a POs-Ca and fluoride combination chewing-gum was given 22 subjects (fluoride group). Every subject chewed two tablets of gum once a day after lunch. The mean $( \pm$ SE) dft and DMFT indices POs-Ca group were $4.6( \pm 0.6)$ and $0.02( \pm 0.02)$ and $4.5( \pm 0.8) 0.0$ in the fluoride group $(\mathrm{p}>0.05)$. The QLF examination found of $4.3( \pm 0.6)$ early caries lesions were detected in POs-Ca group and $3.2( \pm 0.8)$ in the fluoride group. After gum usage $40 \%$ of early caries lesions were recovering in POs-Ca group whereas $72 \%$ of lesions were recovering in the fluoride group ( $\chi$ test; $p<0.001$ ). It was suggested that the chewinggum usage was effective by improvement of the oral health. Especially, chewing-gum containing POs-Ca and fluoride decrease the activity of early caries lesions.

This study was approved by ODU Dental Ethics Committee (\#060716).

\section{4 \\ The Effect of QLF-D in Improving Toothcleaning \\ G.N. Komarov*, C. Anderson, R. Barr, P.W. Smith, S. Brown, S.M. Higham \\ komarovg@liverpool.ac.uk \\ School of Dentistry, University of Liverpool, UK}

The aim of this double blind, single treatment, randomised, four-legged crossover pilot study was to evaluate QLF-D images as a tool to assist participants to improve toothcleaning by providing visual feedback on the extent of plaque accumulation, when compared with standard white light photographic images and disclosing tablets. 12 healthy adult volunteers were recruited for the study after seeking ethical approval. After abstaining from oral hygiene for $48 \mathrm{~h}$ the subjects attended the dental clinic where their front teeth were photographed using QLF-D camera, and the white light and/or fluorescence images were shown to them before and after they were asked to clean their teeth according their protocol. Group one was shown two QLF-D images before and after toothbrushing, group two was shown QLF-D images before toothbrushing, group three (negative control) and group four (positive control) assessed the condition of their plaque accumulation using only white light images before and after toothbrushing. QLF-D images were taken without disclosing the plaque except for the positive control group (four). The results were analysed using $4 \times 4$ repeated measures ANOVA. A trend of decreased $\%$ plaque area coverage was found between the QLF-D group and the negative control demonstrating that subjects appeared to clean their teeth better, if QLF-D images are shown, when compared with only white light images. The difference however was not significant, which is possibly due to small subject numbers in this pilot study. The data from this trial does look promising and will be used to perform power calculations to determine the sample size for future full sized projects.

The study was funded by the University of Liverpool.

\section{5 \\ Effect of Sucrose Concentration on Oral Biofilm Composition: An in vitro Model}

K. Bakht ${ }^{\mathrm{a}}$, S.M. Higham ${ }^{\mathrm{a}}$, E. de Josselin de Jong ${ }^{\mathrm{a}, \mathrm{c}}$, G.C. Martin ${ }^{\mathrm{b}}$, G. Burnett ${ }^{\mathrm{b}}$, C.K. Hope $\mathrm{a}^{\mathrm{a}, *}$

k.bakht@liverpool.ac.uk

aSchool of Dentistry, University of Liverpool, and

${ }^{b}$ GlaxoSmithKline, UK; ' Inspektor Research Systems bv,

Amsterdam, The Netherlands

The presence of cariogenic microorganisms such as mutans streptococci (MS) and lactobacilli together with high carbohydrate consumption has been directly associated with the development of dental caries. Sucrose is considered to be an important cariogenic dietary carbohydrate due to its role in the metabolism of intra- and extracellular polysaccharides by dental plaque microorganisms and its fermentation to acids. Inducing low $\mathrm{pH}$, frequent sucrose fermentation can trigger a clear shift in resident plaque microflora to a more cariogenic state. It is important to fully understand the dynamic nature of the dental plaque community in order to propose useful treatments for caries. This study aimed to investigate the microbial dynamics in biologically relevant biofilm when exposed to differing concentrations of sucrose. Microcosm oral biofilm representative of human plaque was produced using a constant-depth film fermenter (CDFF) and pulsed 8 times daily for $15 \mathrm{~min}$ with $20 \mathrm{mM}$ sucrose solution in one experiment, $100 \mathrm{mM}$ sucrose in another and one remained unpulsed as a control. The biofilm was sampled at day 4, 6, 8, 10, 12 and 14 and bacterial composition enumerated using traditional microbiological culture techniques; Tryptone Yeast Cysteine agar supplemented with sucrose and bacitracin for MS and Rogosa agar for lactobacilli. Results indicate that at $20 \mathrm{mM}$ sucrose exposure MS and lactobacilli achieved pseudo-steady state at $2.79 \times 10^{6} \mathrm{cfu} / \mathrm{mm}^{2}$ and $3.36 \times$ $10^{5} \mathrm{cfu} / \mathrm{mm}^{2}$ (average of day $8-14$ counts, $\mathrm{n}=4$ ) respectively by day 8 . At $100 \mathrm{~mm}$ lactobacilli also achieved steady state by day 8 (average $1.46 \times 10^{6} \mathrm{cfu} / \mathrm{mm}^{2}, \mathrm{n}=4$ ) whereas MS growth was repressed; only achieving $3.36 \times 10^{4} \mathrm{cfu} / \mathrm{mm}^{2}$ at day 8 . In conclusion, differing sucrose concentrations modulated the microbial dynamic of MS and lactobacilli within multispecies biofilm.

This project was funded by BBSRC GSK CASE award. 


\section{6}

Sealant versus Fluoride Varnish for Caries Control in Chilean Children: Randomized Clinical Trial

S. Faleiros ${ }^{\mathrm{a}, *}$, I. Urzua a , C. Díaz ${ }^{\mathrm{b}}$, S. Bangdiwala ${ }^{\mathrm{d}}$, H. Gatica ${ }^{\mathrm{c}}$, R. Cabello ${ }^{a}$, G. Rodríguez ${ }^{\mathrm{a}}$

simone_chioca@yahoo.com.br

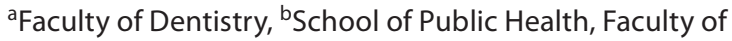
Medicine, and 'Faculty of Medicine, University of Chile, Santiago, Chile; ${ }^{\mathrm{d} D e p a r t m e n t}$ of Biostatistics, University of North Carolina at Chapel Hill, USA

Objective: To compare the effectiveness of sealants versus fluoride varnish within a period of one year in children aged six, of medium-low and low socioeconomic level of Chilean Metropolitan Region, to reduce the incidence of occlusal caries in first molars by a randomized clinical trial. Methods: Of the 441 randomly selected children, 193 were males (43.7\%) and 248 were women (56.2\%) divided into 2 groups (219 to sealants group and 222 to varnish group). With an informed consent of parents and ethics committee approval, the respective interventions were performed at random using block allocation stratified by sex. Information was collected on baseline characteristics of children such as oral hygiene habits, diet, socioeconomic status and caries experience. Results: The average initial DMF was 0.1 (95\% CI: 0.04-0.15) for the sealant group and 0.03 (95\% CI: 0.008-0.06) for the varnish group. The average dmf was 3.3 (95\% CI: 2.9-3.7) for the sealant group and 3.2 (95\% CI: 2.8-3.6) for the varnish group. No statistically significant differences regarding any of the covariates were observed. The multilevel logistic regression model was used for controlling of confounding variables and obtaining the measure of association. As a result of protocol analysis to study the incidence of occlusal caries lesions, an OR of 1.51 (95\% CI: 0.9-2.4) was observed for the sealant group when compared with the varnish group. The previous $\mathrm{dmf}$ experience was the significant covariate in the regression model. The cumulative incidence difference between the groups was $2.8 \%$. Conclusion: There is no evidence to affirm which is the most effective intervention for the control of occlusal caries in the studied population for the first cutoff of the cohort follow-up.

\section{7 \\ The Histologic Gold Standard in Caries Research: A Literature Review \\ P. Bottenberg ${ }^{\text {a, }}$, A.G. Schulte ${ }^{\text {b }}$ \\ pbottenb@vub.ac.be \\ aDepartment of Conservative Dentistry and Prosthodontics, Vrije Universiteit Brussel, Brussels, Belgium; 'bepartment of Conservative Dentistry, Universität Heidelberg, Germany}

Objective: Research in caries diagnosis is related to the use of a gold standard, generally histology. Reproducibility and clarity for the reader make it imperative to explain thoroughly the methodology used. The aim of this review was to assess which methods were used to establish the histological gold standard. Methods: Publications from 1990 to 2010 were retrieved using online databases. Search terms were 'gold standard' OR 'histology' AND 'caries'. Titles and abstracts were used to filter all papers, potentially useful documents were retrieved in full. Inclusion criteria were: text dealing with the gold standard in the diagnosis of natural, primary carious lesions and describing the method. 72 papers were analyzed regarding number of teeth, applied technique (i.e. embedding of teeth, type and thickness of section), number of observers, reproducibility, evaluation system (i.e. number of categories, central depth measurement). Eventually, a variable 'clarity' was derived to judge whether the text would allow reproduction of the study. Results: 79 studies were found, reporting a median of 68 teeth (10-325) per study. Most studies (50) prepared serial sections (50-1,000 $\mu \mathrm{m}$ thickness), followed by hemisection (15). 28 studies mentioned resin embedding of the teeth prior to sectioning, 4 cutting of teeth without embedding; the rest did not mention either procedure. Sections were interpreted by one (15 studies), two ( 22 studies) or more (7 studies) observers. A consensus was reported for most studies employing multiple observers. Reproducibility (e.g. kappa statistics) was rarely reported. Caries was most frequently reported in 3 to 5 categories. 42 studies mentioned criteria to identify a section as carious, 30 did not. Eventually, 14 studies were clearly reported, against 58 not. Clarity of the method was significantly more frequently observed in journals with a higher impact factor $(\mathrm{p}=0.001)$. Conclusion: A more uniform manner of reporting the methods employed as gold standard in caries diagnostic research seems to be desirable.

Clinical research funds TK-VUB.

\section{8 \\ Mothers' Oral Health Profile and Association with Early Childhood Caries in 2-Year-Old Toddlers in Porto}

M. Henriques*, C. Coelho, P. Melo

marisa.trovisco@gmail.com

Department of Conservative Dentistry, Faculty of Dentistry of Porto University, Portugal

Early childhood caries (ECC) is the presence of 1 or more decayed (noncavitated or cavitated lesions), missing (due to caries), or filled tooth surfaces in any primary tooth in a child 71 months of age or younger. Analyzing children's oral health and their mothers may lead understanding which oral characteristics of mothers can put toddlers in risk for ECC. The aim of this study is determine the prevalence of ECC in 2 years toddlers and the role that mothers' oral health profile can play on this disease. A randomized sample of 216 two years old toddlers, living in Porto, was observed, as well their mothers. The visual caries registration followed the WHO criteria for mothers ( $\mathrm{dmft}$ index) with additional recording of initial caries for children (scored taking into account the definition of ECC). A questionnaire was applied to mothers concerning age, scholar degree, employment, annual income, oral hygiene habits, frequency of visits to the dentist and smoking habits. The results showed a dmft value of $0.11 \pm 0.62$ for all children considering initial caries lesion with a prevalence of $3.4 \%$. Considering only dentin lesions, $\mathrm{dmft}$ was $0.06 \pm 0.37$ with a prevalence of $2.3 \%$. Almost all mothers had DMFT $\geq 1$, leading to a prevalence of $99.5 \%$. From those, $72.6 \%$ showed active caries disease $(d \geq 1)$. Of the toddlers with ECC, $89 \%$ had their mothers with active disease, but no children with ECC were found in mothers without active caries. So, absence of disease in the children seems to be dependent of the absence of mothers caries disease $(\mathrm{p}<0.02)$. No statistically significant differences were found in the other vari- 
ables that were observed. This study indicates that the caries status of the mother significantly influences the caries status of her child at 2 years. Although the proportion of dental caries prone children seems small, the relationship between mothers with no active caries and healthy children enhances the importance of not carrying disease during pregnancy and the first years of motherhood.

\section{9}

\section{Chemomechanical Caries Removal with Carisolv ${ }^{\mathrm{TM}}$ :} A Systematic Review of Its Clinical Performance

P. Melo*, M. Henriques, C. Coelho, M.J. Silva, A. Azevedo

paulomelo@netcabo.pt

Department of Conservative Dentistry, Faculty of Dentistry,

University of Porto, Portugal

Minimally invasive dentistry lead to the use of chemomechanical methods which promote the selective softening of carious dentin and an easier removal. It also avoids an excessive tooth structure loss comparing to the traditional dental cavities preparation with rotatory instruments. The aim of this review is to analyze the studies with Carisolv ${ }^{\mathrm{TM}}$, check if it is clinically effective in removing carious tissue and can be considered an alternative to the traditional method. The author undertook a systematic review in PubMed, Cochrane Central Register of Controlled Trials and Science Direct from 1999 till October 2009. The key words were 'chemomechanical caries removal' and 'Carisolv'. The inclusion 'criteria' were clinical randomized controlled trials with Carisolv ${ }^{\mathrm{TM}}$ in one of the study groups; percentage of caries removal, presence of pain, length and patients perception of the treatment. From the 36 studies identified, only 8 fulfilled the inclusion criteria. From the bibliographic references 3 more papers were identified, resulting in a total of 11 . However, only 9 studies used the same criteria to evaluate the efficacy of caries removal of Carisolv ${ }^{\mathrm{TM}}$, which was 85.2 versus $90.1 \%$ of the rotary instrument. The appointment time was greater $5.30( \pm 1.20) \mathrm{min}$, although the time perceived by the patients was 'less' when compared to the conventional technique. From a clinical point of view Carisolv $^{\mathrm{TM}}$ cannot provide a complete removal of caries in all situations, but in cavitated caries, widely open and easily accessible, it shows an efficacy, at least, similar to high speed rotatory instruments.

\section{0 \\ The Extension of Cervical Margins of Class II Restorations and Secondary Caries \\ N.K.Kuper*, N.J.M. Opdam, E.M. Bronkhorst, \\ M.C.D.N.J.M. Huysmans \\ n.kuper@dent.umcn.nl \\ College of Dental Science, Radboud University Nijmegen \\ Medical Centre, The Netherlands}

The aim of this study was to evaluate whether class II restorations with cervical margins apical to the cemento-enamel junction (CEJ) are more prone to failure due to secondary caries than restorations with margins coronal to the CEJ. Records from patients with at least one restoration replaced due to secondary caries were selected from an existing database. Cervical margins of 1,912 approx- imal restoration sites were scored on bitewings in relation to the cemento-enamel junction (coronal to the CEJ and at, or apical to, the CEJ). A method was developed for scoring cervical margin extension on bitewings and validated in vitro. For all restorations the dates of placement, replacement and failure were recorded. Survival times were calculated and a Cox regression analysis was applied to assess the influence of the cervical margin extension on survival of the restorations. Hazard ratios were calculated. Of the 1,912 restoration sites examined, 655 failed, 399 restorations with margins above the CEJ and 256 with margins below or at the CEJ. 257 restorations failed because of secondary caries. The extension of the cervical margin did not significantly influence failure due to secondary caries. In conclusion the extension of restorations at the cervical margin had no significant effect on the development of secondary caries.

The study was supported by the authors' institution.

\section{$40 a$ \\ Cost-Effectiveness of Stepwise Excavation/Partial Removal of Carious Dentine in Deep Caries Lesion in Brazil \\ J.J. Jardim ${ }^{\mathrm{a}, *}$, R. Decourt ${ }^{\mathrm{a}}$, L.M. de Paula ${ }^{\mathrm{b}}$, H.D. Mestrinho ${ }^{\mathrm{b}}$, M. Maltz ${ }^{\mathrm{a}}$ \\ jujobim@yahoo.com \\ a Federal University of Rio Grande do Sul, Porto Alegre, RS, and \\ b Brasilia University, Brasilia, DF, Brazil}

In a multicenter randomized controlled clinical trial, two treatments for deep caries lesions in permanent molars were analyzed to determine therapy success rates after one year. The stepwise excavation technique (SW) was compared to partial removal of carious dentin in one session (PDR) in public health services in Brazil. After one year of treatment performance, 180 restorations had been evaluated, showing $97.9 \%$ and $74.1 \%$ success in PDR and SW groups respectively $(\mathrm{p}<0.000)$. The aim of the present study was to determine the cost-effectiveness of these two treatments. The discount cash flow method (DCF) was adopted. The total cost of each treatment was calculated using: filling material; $x$-ray exam; number of faces involved in the restoration; the cost of the failures (endodontic treatment) for each group was added to the cost calculation according to the success rates from the one year follow-up. To calculate the economic value of PDR, a public health service unit composed by three dentists in four hour work shifts was used, assuming a total of 528 treatments/month. The prevalence of deep caries lesions in permanent molars was set in $5 \%$. The PDR provides an economy of $67.78 \%$ per treatment compared to SW. The overall economy in the annual cost of the public health center is $2.39 \%$. These results showed that PDR is a much more efficient treatment than SW after one year of follow-up. In conclusion, performing the partial removal of carious dentin in one session, which presents a higher rate of success and a high economic value, generates benefits for the public finances (direct economy), for the public health services (increase in number of treatments performed) and for the patients (comfort and time).

Funding sources: Grants: CAPES/Ministry of Education, CNPq/Ministry of Science and Technology (403420/04-0) and FAPERGS (04/1531-8). Financial support from industries: Ivoclar/Vivadent (Liechtenstein), DFL (Brazil), and SDI (Australia). 


\section{Caries Research}

\section{Session 3 \\ De- and Remineralization}

\section{1 \\ A Multi-Step Model for the Dissolution Mechanism of Calcium Apatites in Acids \\ S.V. Dorozhkin \\ sedorozhkin@yandex.ru \\ Kudrinskaja sq. 1-155, Moscow 123242, Russia}

A complete dissolution mechanism of calcium hydroxyapatite (HAp) in acids is the key for the understanding of progression of mineral loss of enamel in caries but there is no definitive model. The aim of this study was to analyse previously published models of HAp dissolution to identify the key aspects of each and combine these to produce an overall general physico-chemical model of HAp dissolution. Eight different models of HAp reacting with acids were critically reviewed. The identifying features were extracted from each model and used to generate a general multistep model incorporating the key features of each. HAp dissolution is a series of distinct identifiable steps of chemical kinetic and thermodynamic reaction processes, involving the reaction of acids with HAp surfaces. These steps include (1) diffusion of acids from the bulk solution through the Nersnt layer, (2) their adsorption onto the surface, (3) chemical transformations on the surface, (4) desorption of the reaction products, (5) their diffusion through the Nersnt layer to the bulk solution. All the steps mentioned appear to be much more complex. For example, processes (1) and (5) include chemical transformations happen with the ions during diffusion, because solution $\mathrm{pH}$ is known to depend on the distance from the solid/liquid interface (in acidic solutions it is higher near the surface of apatite and decreases when the distance increases). Processes (2) and (4) include ionic diffusion along the surface both to (step $2^{\mathrm{a}}$ ) and away from (step $4^{\mathrm{a}}$ ) the crystal steps, respectively, as well as a diffusive jump (steps $2^{\mathrm{b}}$ and $4^{\mathrm{b}}$ ). Finally, process (3) consists of several successive chemical transformations (step $3^{\mathrm{a}}$ ) and includes ionic detachment from the kink sites $\left(\right.$ step $3^{\text {b }}$ ). Moreover, for adsorption (step 2) to take place, the fol- lowing intermediate steps are necessary: (i) dehydration of a surface site, (ii) partial dehydration of ions and (iii) a diffusive jump toward the surface. One can also expect three similar intermediate steps (diffusion jump from the surface, hydration both the ions and the surface site) for desorption (step 4). It is clear that some of these steps require further experimental investigation. In conclusion a general description of HAp dissolution requires a multi-step model of the individual diffusion and reaction steps.

Supported by ORCA Conference Travel Fellowship.

\section{2 \\ Inhibition of Dentine Collagen Degradation in situ by Hesperidin \\ T. Takatsuka ${ }^{\mathrm{a}, *}$, Y. lijima $^{\mathrm{b}}$, R. Sono ${ }^{\mathrm{a}}$, A.J.P. van Strijp ${ }^{\mathrm{c}}$ \\ tsutomu.takatsuka@jp.sunstar.com \\ aSunstar, Osaka, and b Nagasaki University Graduate School, Nagasaki, Japan; 'Academic Centre for Dentistry Amsterdam (ACTA), The Netherlands}

Dentine caries is a process of demineralisation and subsequent degradation of the organic matrix (mainly collagen type I) by matrix metalloproteinases (MMPs). The aim of this spilt-mouth study was to assess whether hesperidin (HPN) could inhibit dentine collagen degradation. Seventeen healthy volunteers received two completely demineralised dentine specimens placed contralaterally in the buccal flange of their mandibular partial denture. The specimens were covered by Dacron gauze to enhance plaque accumulation. Complete demineralisation was achieved by incubation in $0.1 \mathrm{M}$ acetic acid during 9 days. The participants were instructed to immerse each specimen extraorally in the randomly assigned solution (HPN suspension (1,000 ppm) as test solution; distilled water as placebo solution) twice daily during $3 \mathrm{~min}$. After 
the experimental period of 4 weeks the specimens were collected, solubilized with bacterial collagenase type VII (SIGMA CO773$3 \mathrm{KU}$ ) and hydrolyzed in $6 \mathrm{~N}$ hydrochloric acid. The collagen content was determined by amino acid analysis (hydroxyproline content). Predefined exclusion criteria included low or non responders $(<20 \%$ collagen loss in the in situ control specimens compared to the in vitro control specimens $(\mathrm{n}=10$; mean collagen content $2.35 \pm 0.27 \mathrm{mg} / \mathrm{specimen}))$. The results $(\mathrm{n}=13)$ showed that the mean dentine collagen content in the test group $(1.67 \pm 0.68 \mathrm{mg} /$ specimen) was statistically significantly larger than in the placebo group $(1.35 \pm 0.57 \mathrm{mg} / \mathrm{specimen}$; paired t test; $\mathrm{p}<0.02)$. This in situ study shows that hesperidin could inhibit degradation of dentine collagen.

This study was supported by grant from Sunstar.

\section{3}

\section{Anticaries Potential of an RMGI Coating on Dentin}

R.P. Rusin ${ }^{\mathrm{a}, *}$, D. Tantbirojn ${ }^{\mathrm{b}}$, A. Versluis $^{\mathrm{b}}$, D.G. Augustson ${ }^{\mathrm{c}}$, N.C. Kumar ${ }^{\mathrm{a}}$, A.M. Pfarrer ${ }^{\mathrm{a}}$

rprusin@mmm.com

a3M ESPE, St. Paul, Minn., bUniversity of Tennessee, Memphis, Tenn., and 'University of Minnesota, Minneapolis, Minn., USA

The aim was to determine the anticaries potential on dentin of a resin-modified glass ionomer (RMGI) coating material (3M $\mathrm{M}^{\mathrm{TM}}$ ESPE $^{\text {TM }}$ Vanish $^{\text {TM }}$ XT, VXT) versus a flowable composite as negative control (3M $\mathrm{M}^{\mathrm{TM}}$ ESPE ${ }^{\mathrm{TM}}$ Filtek $^{\mathrm{TM}}$ Supreme Plus Flow, FSPF). The radiopacity of the flowable allowed it to be distinguished from dentin, in contrast to unfilled or silica-filled sealants. Rectangle slots $(6 \times 2 \times 0.5 \mathrm{~mm})$ were prepared in polished bovine dentin specimens with a \#245 carbide bur ( $\mathrm{n}=8$ per group). FSPF was applied to the dentin slot after etching and application of adhesive ( $3 \mathrm{M}^{\mathrm{TM}}$ ESPETM Single Bond Plus); VXT was applied either directly to the unetched dentin or after $30 \mathrm{~s}$ phosphoric acid etch. The tooth surface was painted with acid-resistant nail polish except a window of dentin next to the coating materials. Specimens were soaked 21 days at $37^{\circ} \mathrm{C}$ in $0.1 \mathrm{M}$ lactic acid gel as simulated cariogenic challenge. Transverse microradiography of the dentin lesion was used to determine the width of the zone of inhibition (ZOI), and mineral loss (DZ) at 0.25 and $1 \mathrm{~mm}$ from coating. Data were analyzed via one-way ANOVA and compared with Tukey's $\mathrm{t}$ test $(\mathrm{p}<0.05)$. The ZOI (micrometers, mean $(\mathrm{SD})$ ), DZ at $0.25 \mathrm{~mm}$ (volume $\%$ mineral-micrometer, mean (SD)), and DZ at $1.0 \mathrm{~mm}$ (volume\% mineral-micrometer, mean (SD)) were for FSPF 0 (0), 6,918 (763), 6,483 (830); VXT-unetched 39 (9), 2,956 (991), 3,589 (828); VXT-etched 42 (12), 2,939 (603), 3,647 (655). The ZOI of VXT on either etched or unetched dentin was significantly greater than for the FSPF control. DZ up to $1 \mathrm{~mm}$ away from VXT on either etched or unetched dentin was significantly less than for FSPF. VXT exhibited greater protection against dentin demineralization than the negative control both adjacent to the coating and up to $1 \mathrm{~mm}$ away. It is proposed that this extended protection against the simulated cariogenic challenge is due to bioavailable fluoride, calcium, and phosphate released from VXT.

Supported in part by a $3 \mathrm{M}$ Non-tenured Faculty Grant, and by 3M ESPE.

\section{4 \\ A Comparison of Terahertz Pulsed Imaging with Transverse Microradiography for the Assessment of Enamel Remineralisation}

D. Churchley ${ }^{\mathrm{a},}$, R.J.M. Lynch ${ }^{\mathrm{a}}$, J. Alton ${ }^{\mathrm{b}}$, A. Hara ${ }^{\mathrm{C}}$, F. Lippert ${ }^{\mathrm{c}}$

david.r.churchley@gsk.com

aGlaxoSmithKline, Weybridge, and bTeraview Limited,

Cambridge, UK; ${ }^{c}$ Oral Health Research Institute, Indianapolis, USA

Transverse micro-radiography (TMR) is the 'gold standard' for measuring mineral changes in enamel; however, it is destructive in nature. The aim of this study was to compare a non-destructive technique, Terahertz Pulsed Imaging (TPI), with TMR for measuring remineralisation of caries lesions. Bovine enamel blocks were immersed in $0.1 \mathrm{M}$ lactic acid containing $0.2 \%$ Carbopol C907, 50\% saturated with hydroxyapatite, $\mathrm{pH} 5.0$ for $72 \mathrm{~h}(\mathrm{n}=$ 12 per treatment group). The 20 day experimental protocol consisted of four daily, one-minute treatment periods (1 part dentifrice: 2 parts saliva, a $4 \mathrm{~h}$ /day acid challenge, and $20 \mathrm{~h} /$ day remineralisation period (50:50 pooled human/artificial saliva mixture). The treatment dentifrices were: (A) 0 ppm NaF, (B) 675 ppm NaF, (C) 1,400 ppm NaF, (D) 1,100 ppm MFP/450 ppm NaF and (E) $1,400 \mathrm{ppm}$ AmF. All specimens were imaged using a TPI imaga 1000. The baseline and post-treatment refractive index profiles were extracted and the differences in the integrated areas across the depth of the lesion were used to calculate $\Delta \Delta \mathrm{Z}_{\mathrm{TPI}}$ (units $=$ vol $\%$ $\times \mu \mathrm{m})$. The change in lesion depth (LD) (units $=\mu \mathrm{m}$ ) was also determined. $\Delta \Delta \mathrm{Z}_{\mathrm{TMR}}$ and LD were measured subsequently using TMR. $\Delta \Delta \mathrm{Z}_{\mathrm{TMR}} / \Delta \Delta \mathrm{Z}_{\mathrm{TPI}}$ values (SE in brackets) were (A) -740 (47)/-569 (38), (B) 490 (49)/357 (40), (C) 700 (47)/620 (38), (D) 46 (50)/-4 (40), (E) $275(49) / 190(39) . \Delta \mathrm{LD}_{\mathrm{TMR}} / \Delta \mathrm{LD}_{\mathrm{TPI}}$ values (SE in brackets) were (A) -19.2 (1.4)/-16.9 (1.3), (B) 7.2 (1.5)/3.9 (1.4), (C) 10.7 (1.5)/14.9 (1.3), (D) -6.3 (1.5)/-7.0 (1.4), (E) 4.8 (1.5)/1.5 (1.4). Significant $(\mathrm{p}<0.05)$ linear correlations between $\Delta \Delta \mathrm{Z}_{\mathrm{TMR}}$ and $\Delta \Delta \mathrm{Z}_{\mathrm{TPI}}$, and $\Delta \mathrm{LD}_{\mathrm{TMR}}$ and $\Delta \mathrm{LD}_{\mathrm{TPI}}$ (Pearson) were observed for each treatment group. These findings demonstrate that TPI could be a useful technique for non-destructive assessment of enamel remineralisation.

\section{5}

The Peptide Chain Length Required for Statherin-Like Peptide Hydroxyapatite Demineralization Inhibition Efficacy

S. Shah, J. Kosoric, M.P. Hector, P. Anderson*

p.anderson@qmul.ac.uk

Centre for Oral Growth and Development, Institute of Dentistry, Barts and The London School of Medicine and Dentistry, Queen Mary University of London, UK

The salivary protein statherin (StN43) and its shortened Nterminal analogue StN21 reduces the kinetics of enamel and hydroxyapatite (HAp) demineralization. In order to understand 
the molecular mechanisms involved, the aim of this study was to measure the cariostatic efficacy of shorter length statherin-like peptides to ascertain the minimum peptide length required for function. Statherin-like peptides containing the N-terminal 5, 10,15 and 21 residues respectively were prepared using solidstate FMOC synthesis and dissolved in phosphate buffer at $\mathrm{pH}$ $7.4(0.188 \mathrm{~mm})$. A control solution containing only phosphate buffer was also prepared. HAp pellets (20\% porosity) used as model substrates for enamel demineralisation studies were sectioned and mounted in scanning microradiography (SMR) environmental cells, and exposed to a $0.1 \mathrm{M}$ acetic acid at $\mathrm{pH} 4.0 \mathrm{de}-$ mineralization solution for $120 \mathrm{~h}$. The sections were then treated with one of the peptide solutions for $24 \mathrm{~h}$. The sections were then exposed to the demineralization solution for a further $120 \mathrm{~h}$. SMR was used to measure the rate of mineral loss in each section before and after exposure to the peptide solution. The HAp sections treated with StN21 and StN15 peptides demonstrated an approximately $45 \%$ decrease in rate of mineral loss following treatment, whereas those treated with StN10, StN5 peptides and the control, demonstrated no decrease in rate of mineral loss following treatment. Statherin-like peptides of N-terminal length of 15 residues or greater are required for cariostatic efficacy. Peptides of N-terminal length of 10 or less residues do not have cariostatic function. This may be related to the strong hydrogen bonding and van der Waals interactions of the N-terminal Arg13 residue reported from computational studies of statherin binding to hydroxyapatite [Makrodimitris et al.: J Am Chem Soc 2007;129:13713-13722].

Oral and Dental Research Trust.

\section{6 \\ The Effect of a Remineralising Agent on Developmentally Hypomineralised Enamel}

F.A. Crombie*, D.J. Manton, J. Palamara, E.C. Reynolds

djmanton@unimelb.edu.au

Melbourne Dental School, University of Melbourne, Melbourne, Australia

Molar hypomineralisation (MH) is a developmental enamel defect with a prevalence of up to $40 \%$ and associated with many problems ranging from extreme sensitivity impeding normal function and significantly higher levels of treatment need, dental anxiety and poor prognosis. Remineralising agents are used widely for MH management however virtually no supporting evidence exists, and none using the gold standard of transverse microradiography (TMR). The aim of this pilot in vitro study was to investigate the effects of exposing enamel in teeth affected by $\mathrm{MH}$ to remineralising agents. Hypomineralised lesions $(H L ; n=6)$ were partly covered with varnish to leave an area of the lesion exposed. Prior to varnishing, the lesions were pre-treated by polishing with abrasive discs with or without $0.95 \% \mathrm{w} / \mathrm{v} \mathrm{NaOCl}$ irrigation. The partly covered lesions were then exposed to $1 \% \mathrm{w} / \mathrm{v}$ casein phosphopeptide-amorphous calcium fluoride phosphate (CPP-ACFP) solution, pH 5.5 for 14 days with solutions changed daily. Lesion sections, composing remineralisation and control halves, were lapped to $\sim 100 \mu \mathrm{m}$ for TMR. Initial lesions varied considerably (mineral deficiency ranged from 21.4 to $40.5 \%)$. \%R ( $\Delta$ Zcontrol $-\Delta$ Zremineralisation $) / \Delta$ Zcontrol $\times 100)$ values, representing proportional mineral content gains considering the full depth of the lesions for each group were: polish group $18.6 \pm 8.1 \%$, polish $+\mathrm{NaOCl}$ group $14.1 \pm 4.0 \%$. Changes also differed according to depth from the surface: at $0-50 \mu \mathrm{m} \% \mathrm{R}=$ polish group $48.0 \pm 20.6 \%$, polish $+\mathrm{NaOCl}$ group $23.6 \pm 7.9 \%$; at $0-100 \mu \mathrm{m}$ $\% \mathrm{R}=$ polish group $35.0 \pm 5.4 \%$, polish $+\mathrm{NaOCl}$ group $26.7 \pm$ $11.0 \%$. NaOCl pre-treatment did not significantly increase remineralisation. These pilot results illustrate the great variability of hypomineralised lesions and also indicate that long-term exposure (at $\mathrm{pH} 5.5$ for 14 days) to CPP-ACFP increased the mineral content of developmentally hypomineralised enamel.

Dentsply Australia and Melbourne Dental School Research Committee.

\section{7 \\ Effect of $\mathrm{CO}_{2}$ Laser $(10.6 \mu \mathrm{m})$ Irradiation at $2 \mathrm{~J} / \mathrm{cm}^{2}$ on Caries Prevention in vivo \\ M. Esteves-Oliveira ${ }^{a}$ * , H. Pollheim a , F. Lamperta, C.P. Eduardoc, G. Conrads ${ }^{\mathrm{b}}$, C. Apel $^{\mathrm{a}}$ \\ marcella@usp.br \\ aDepartment of Operative Dentistry, Periodontology and Preventive Dentistry, and bivision of Oral Microbiology and Immunology, RWTH Aachen University, Aachen, Germany; 'Department of Restorative Dentistry, University of São Paulo (USP), São Paulo, Brazil}

The aim of the study was to evaluate the potential of $\mathrm{CO}_{2}$ laser irradiation to prevent caries in an animal model. 48 pathogen-free Sprague-Dawley rats aged 23 days were obtained and divided into 4 groups $(n=12)$ receiving different treatments: no treatment, as negative control group (C); laser irradiation (L); application of fluoride (F); and combined fluoride and laser treatment (FL). Treatments were performed on day 7 using a pulsed $\mathrm{CO}_{2}$ laser at $1.8 \mathrm{~J} / \mathrm{cm}^{2}(226 \mathrm{~Hz}, 5 \mu \mathrm{s})$ and a NaF varnish (5\% F). The F group received the second fluoride application on day 28. Suppression of indigenous flora was performed by administration of chlorhexidine-digluconate $(0.12 \%)$ on day 2 , and amoxicillin/clavulanic acid (300/75 mg/kg/day) on day 1 . A cariogenic diet: $63 \%$ sucrose and water with $5 \%$ of sucrose were provided ad libitum. During the first three experimental days the animals received oral inoculation of $2 \times 10^{9}$ Streptococcus mutans (UA159) suspension. On the 56th day animals were killed and caries was scored by the methods of Keyes. Microbiological evaluation was performed through real time quantitative PCR addressing the S. mutans glycosyltransferase gene. Statistical analysis: ANOVA and Tukey post-hoc tests $(\alpha=0.05)$. All treatments resulted in significantly lower mean caries score in enamel than the control group $(\mathrm{p}<$ $0.05)$. For the score indicating slight dentin involvement only group F resulted in significantly lower mean than all other groups $(\mathrm{p}<0.05)$. S. mutans colonization was statistically significantly reduced after laser irradiation $(p<0.05)$ but after 21 days an increase was observed and number of cells was not significant dif- 
ferent form other groups until the end of the experiments. Laser irradiation could decrease enamel caries formation in vivo (26\%). However, this decrease was lower than observed after two fluoride varnish applications.

START Program of the Medical Faculty of RWTH Aachen University (grants number AZ43/09).

\section{8 \\ Effect of Calcium-Containing Chewing Gums on Subsurface Lesion Remineralisation in situ}

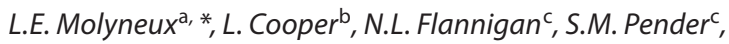
C.K. Hope ${ }^{\mathrm{b}}$, P.W. Smith ${ }^{\mathrm{a}}$, N. Pender ${ }^{\mathrm{b}}$, C.C. Youngson ${ }^{\mathrm{a}}$, S.M. Higham ${ }^{\mathrm{b}}$

L.E.Molyneux@liv.ac.uk

Departments of a Restorative Dentistry, ${ }^{\mathrm{b}}$ Oral Biology and

'Orthodontics, University of Liverpool, UK

The aim of the study was to determine the effects of two experimental calcium-containing, sugar-free chewing gums in comparison with a placebo gum and no gum phase on enamel remineralisation in situ. Artificial subsurface caries lesions were prepared on buccal surfaces of human premolars coated with acid resistant nail varnish leaving exposed enamel windows $(9 \times$ $2 \mathrm{~mm}$ ) by immersion in demineralising gel $(0.1 \mathrm{M}$ lactic acid, $0.1 \mathrm{M}$ sodium hydroxide, $\mathrm{pH} 4.5)$ for 7 days. Lesions were sectioned to give three slabs $(3 \times 2 \mathrm{~mm})$, two for use in an in situ appliance, and one retained to determine baseline lesion parameters using transverse microradiography (TMR). Enamel slabs were covered with gauze and secured within a modified orthodontic bracket. Healthy adult volunteers $(n=18)$ were recruited and randomly assigned a subject number and treatment sequence using a Latin square. A bracket housing one of the enamel slabs was bonded to the buccal surface of a lower molar and worn for 28 days per experimental phase. Following removal of the appliance and a 14 day washout period, subsequent cycles were undertaken. Each participant completed all 4 experimental phases. Compliance was checked following each study phase using a questionnaire and was reportedly high. Enamel slabs were sectioned and polished to give planoparallel sections $80 \mu \mathrm{m}$ thick. Mineral content $(\Delta \mathrm{Z})$, lesion depth (Ld) and lesion width (Lw) were quantified using TMR. The mean percentage mineral gain $(\Delta \mathrm{Z})$ was highest with the placebo gum A (41.2 \pm 10.0$)$, followed by experimental gums B $(35.2 \pm 21.9)$ and C (30.9 \pm 16.7$)$, and no gum $(17.9 \pm 26.9)$. Tukey's a posteriori confirmed significant differences between gums $\mathrm{A}$ and $\mathrm{B}$ but not $\mathrm{C}$ when compared with no gum. No significant differences were identified between the gums. This study showed that remineralisation occurred with all gums, however the calcium-containing gums did not produce more remineralisation than the placebo gum.

This study was supported by the William Wrigley Jr. Company.

\section{9 \\ Evaluation of Fluorosed Teeth Indicated for Orthodontics after Application of Resin Infiltration Material}

\author{
I.M.G. ElZayat ${ }^{\mathrm{a},}$ *, M. El Banna ${ }^{\mathrm{a}}$, S.M. Hammad ${ }^{\mathrm{b}}$ \\ salah_mai@yahoo.com \\ aOperative Department, Misr International University, and \\ ${ }^{b}$ Department of Orthodontic, Mansoura University, Cairo, Egypt
}

The aim of this study was to examine the surface texture of fluorosed teeth indicated for orthodontics before and after application of resin infiltration materials. Twenty fluorosed premolar teeth were divided into four equal groups. Group 1: teeth were exposed to $\mathrm{pH}$ and thermocycling aging (Featherstone, 1996) and ICON material was applied, group 2 same as group 1 then the brackets were applied, group 3 same as group 2 but without application of the ICON material, group 4 teeth subjected to thermocycling aging. After two weeks of aging, the brackets were removed from the teeth in groups 2 and 3. For all the teeth of the 4 groups, microhardness (as $\mathrm{kgf} / \mathrm{mm}^{2}$ ) was determined using a Vicker indentor (100 g per $10 \mathrm{~s}$ ) on standardized selected points on the labial surfaces. Digital images were obtained and analysed for all teeth from the four groups to investigate the color changes between the groups on a scale graded from black to white, also the surface roughness were investigated using 3 dimensional presentation software. ANOVA and Duncan's multiple range test were used to compare mean values. There was a significant difference in the surface hardness of fluorosed teeth with and without application of ICON material (group 1 and 4), the surface hardness reached $224( \pm 18.4)$ after the application of ICON and $176( \pm 12.3)$ after aging. Also, significant difference between group 3 and 2 in the surface hardness was observed as it was $217( \pm 15.2)$ in group 2 and $157( \pm 10.8)$ in group 3 . No significant difference was observed between group 1 and 2. Image analysis showed differences between fluorosed teeth with and without ICON application. The application of brackets on fluorosed teeth could have no harmful effect if resin infiltration material was applied before their fixation.

Misr International University and Mansoura University.

\section{0 \\ Calcium, Phosphate, Degree of Saturation and Hydroxyapatite Dissolution \\ J. Jalili*, S.B. Jones, N.X. West, M.E. Barbour \\ Jennifer.Jalili@UHBristol.nhs.uk \\ School of Oral and Dental Sciences, University of Bristol, Bristol, UK}

The rate of dissolution of dental enamel in acidic solution is dependent on a variety of factors including $\mathrm{pH}$, acid concentration and degree of saturation with respect to hydroxyapatite $\left(\mathrm{DS}_{\mathrm{HA}}\right)$. Although it has been suggested that $\mathrm{DS}_{\mathrm{HA}}$ can be used as a predictive parameter in demineralisation models, it was shown more recently that for a given $\mathrm{DS}_{\mathrm{HA}}$, individual calcium and phosphate concentrations also affect the rate of enamel dissolution. 
The aim of this study was to characterise the relationship between hydroxyapatite dissolution and calcium and phosphate concentration, for a given $\mathrm{DS}_{\mathrm{HA}}$. The motivation was to develop a better understanding of the thermodynamic forces underlying dental erosion and to develop improved methods for combating this destructive and potentially aggressive condition. A pH stat was used to measure dissolution of hydroxyapatite discs over $30 \mathrm{~min}$ periods in citric acid solutions $\left(\mathrm{pH} 3.20,37^{\circ} \mathrm{C}\right)$, all with $\mathrm{DS}_{\mathrm{HA}}=0.100$ but different calcium and phosphate concentrations. Concentrations, expressed as $\left[\mathrm{Ca}^{2+}\right] /\left[\mathrm{PO}_{4}{ }^{3-}\right]$ in $\mathrm{mmol} / \mathrm{l}$, were $68 / 1,27 / 3,17 / 5$, $10 / 10$ and $6 / 28$. Solutions with $\left[\mathrm{Ca}^{2+}\right]>\left[\mathrm{PO}_{4}{ }^{3-}\right](68 / 1,27 / 3,17 / 5)$ resulted in very similar hydroxyapatite dissolution rates. Dissolution was more rapid with $\left[\mathrm{Ca}^{2+}\right]=\left[\mathrm{PO}_{4}{ }^{3-}\right](10 / 10)$, but this was not a statistically significant difference as indicated using a one-way ANOVA with Tukey's HSD. Dissolution was faster still with $\left[\mathrm{Ca}^{2+}\right]<\left[\mathrm{PO}_{4}{ }^{3-}\right](6 / 28)$; this rate was significantly faster than solutions $68 / 1,27 / 3,17 / 5$ but not $10 / 10$. In conclusion, dissolution of hydroxyapatite discs was significantly $(\mathrm{p}<0.05)$ affected by the ratio of calcium to phosphate in solutions with $\mathrm{DS}_{\mathrm{HA}}=0.100$, but only where $\left[\mathrm{Ca}^{2+}\right]<\left[\mathrm{PO}_{4}{ }^{3-}\right]$. When $\left[\mathrm{Ca}^{2+}\right] \geq\left[\mathrm{PO}_{4}{ }^{3-}\right]$ there was no significant difference between dissolution rates. This has significance for the understanding of dental erosion and the design of products intended to reduce dental erosion.

School of Oral and Dental Sciences, University of Bristol.

\section{1 \\ Effect of Zinc lons $\left(\mathrm{Zn}^{2+}\right)$ on Hydroxyapatite Dissolution Kinetics Studied Using Scanning Microradiography \\ H. Lingawi ${ }^{\mathrm{a}, *}$, M. Barbour ${ }^{\mathrm{b}}$, R.J.M Lynch ${ }^{\mathrm{c}}$, P. Anderson ${ }^{\mathrm{a}}$ \\ h.lingawi@qmul.ac.uk \\ ${ }^{a}$ Department of Dental Physical Sciences, Center of Oral Growth and Development, Institute of Dentistry, Queen Mary University of London, ${ }^{b}$ Department of Dental Materials Science \& Biomaterials, School of Oral \& Dental Sciences, University of Bristol, and ${ }^{\mathrm{C}}$ GlaxoSmithKline, Weybridge, UK}

Zinc $\left(\mathrm{Zn}^{2+}\right)$ is a dietary essential trace element necessary for various body functions. It is used in toothpaste for its anti-calculus properties and reducing oral malodour, but it may also have a role in inhibiting dissolution kinetics of enamel's principal inorganic component; hydroxyapatite (HAP). The aim of this study was to investigate the effect of $\mathrm{Zn}^{2+}$ on surface physical chemistry influencing HAP dissolution by measuring the rate of HAP dissolution $\left(\mathrm{RD}_{\mathrm{HAP}}\right)$ under strictly controlled thermodynamic conditions relevant to caries and erosion using scanning microradiography (SMR) at a range of $\mathrm{Zn}^{2+}$ concentrations. Compressed sintered HAP discs (Plasma-Biotal, UK) were coated with acidresistant varnish on all surfaces except one, and located in an SMR cell. A bulk solution of $0.1 \%$ acetic acid $\mathrm{pH} 4$, divided into five

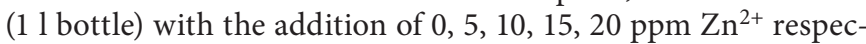
tively was prepared. $0.3 \%$ citric acid $\mathrm{pH} 2.8$ solutions were similarly prepared. The demineralising solution was circulated at 0.80 $\mathrm{cm}^{3} \cdot \mathrm{min}^{-1}$, and the $\mathrm{RD}_{\mathrm{HAP}}$ was measured using SMR at a single centrally located point on each disc for $24 \mathrm{~h}$ at $22^{\circ} \mathrm{C}$. Each experiment was repeated in triplicate for both increasing, and decreas- ing, $\mathrm{Zn}^{2+}$ concentrations. For acetic acid, the mean $\mathrm{RD}_{\mathrm{HAP}}$ decreased significantly $(\mathrm{p}<0.05)$ from $3.08 \times 10^{-3}$ (with no $\mathrm{Zn}^{2+}$ added) to $2.87 \times 10^{-3}, 2.74 \times 10^{-3}, 2.42 \times 10^{-3}$ and $2.04 \times 10^{-3} \mathrm{~g}$ $\cdot \mathrm{cm}^{-2} \cdot \mathrm{h}^{-1}$ at $\mathrm{Zn}^{2+}$ concentrations of $5,10,15$ and $20 \mathrm{ppm}$ respectively. For citric acid, the mean $\mathrm{RD}_{\mathrm{HAP}}$ decreased significantly $(\mathrm{p}<0.05)$ from $4.7 \times 10^{-4}$ (with no $\mathrm{Zn}^{2+}$ added) to $4.0 \times 10^{-4}$, $3.13 \times 10^{-4}, 2.91 \times 10^{-4}$, and $2.84 \times 10^{-4} \mathrm{~g} \cdot \mathrm{cm}^{-2} \cdot \mathrm{h}^{-1}$ at $\mathrm{Zn}^{2+}$ concentrations of $5,10,15$ and $20 \mathrm{ppm}$ respectively. This study demonstrates that $\mathrm{Zn}^{2+}$ decreased $\mathrm{RD}_{\mathrm{HAP}}$ under strictly controlled thermodynamic conditions relevant to caries and erosion, possibly due to inhibition of dissolution nuclei on the HAP surfaces. bia.

Saudi Ministry of Higher Education, Kingdom of Saudi Ara-

\section{2 \\ Efficacy of CPP-ACP and CPP-ACFP in Patients with Salivary Gland Hypofunction: An in situ Study \\ T. Peric ${ }^{\mathrm{a}}$ *, B. Petrovic ${ }^{\mathrm{b}}$ \\ tamara.peric@stomf.bg.ac.rs \\ ${ }^{a}$ Faculty of Dentistry, University of Belgrade, and \\ ${ }^{b}$ Dentistry Clinic of Vojvodina, University of Novi Sad, Serbia}

A general attitude is that additional use of $0.05 \% \mathrm{NaF}$ solutions together with fluoridated dentifrices presents an adequate preventive measure in xerostomic patients. Recently, novel calciumphosphate-based systems have been developed to assist remineralisation. The purpose of this in situ study was to compare the efficacy of casein phosphopeptide-amorphous calcium phosphate (CPP-ACP) and casein phosphopeptide-amorphous calcium fluoride phosphate (CPP-ACFP) with that of $0.05 \% \mathrm{NaF}$ among individuals with salivary gland hypofunction. The study comprised 30 patients aged $15-54$ years randomised into three groups $(n=10)$ : CPP-ACP, CPP-ACFP, and $0.05 \% \mathrm{NaF}$, to be used two times a day according to the manufacturers' instructions. The included patients suffered from medical condition associated with xerostomia, had symptoms of dry mouth for longer than 6 months, and the stimulated salivary flow rate lower than $0.7 \mathrm{ml} / \mathrm{min}$. Demineralised enamel slabs were mounted for the 28-days experimental period. Remineralisation potential was examined using scanning electron microscope (SEM) and energy dispersive spectroscopic (EDS) technique. SE microphotographs were subsequently analysed with image analysis software. Parameters that were analysed included area, minimal, maximal and mean diameter and roundness of enamel defects. Treatment with $0.05 \% \mathrm{NaF}$ reduced the number and the appearance of enamel defects to some extent when compared with irregular demineralised enamel. Treatment with CPP-ACP and CPP-ACFP resulted in occlusion of defects which produced more flattened enamel surface with less significant defects. There was a statistically significant difference between the treatment groups $(\mathrm{p}<0.001)$ regarding the area and diameter of enamel defects. The EDS analysis did not show significant differences in $\mathrm{Ca} / \mathrm{O}, \mathrm{P} / \mathrm{O}$ and $\mathrm{Ca} / \mathrm{P}$ ratios between the groups $(\mathrm{p}>0.05)$. $\mathrm{CPP}-\mathrm{ACP}$ and CPP-ACFP hold promise as caries preventive agents for patients with salivary gland hypofunction.

GC Int. (Tokyo, Japan) and Curaden International AG (Kriens, Switzerland) provided the materials for this study. 


\section{3}

\section{Prevention of Enamel Demineralization around Orthodontic Brackets Using an Organo-Diselenide Antimicrobial Sealant}

B. McGarrell, P. Mensinkai, I. Chedjieu, P.T. Gakunga, J.P. Hatch, K. Ramalingam, B.T. Amaechi*

amaechi@uthscsa.edu

University of Texas Health Science Center at San Antonio, USA

The objective of this study was to determine if a diselenidecontaining (SeLECT Defense) prime or sealant can prevent enamel demineralization, and compare it to that of other available products. Selenium containing compounds act as catalyst in the formation of superoxide radicals with an extremely short half-life and path length, resulting in an environment toxic to bacteria yet safe for humans. 105 tooth halves with either buccal or lingual surface were produced, and orthodontic brackets were bonded on each surface. The remaining surface of each tooth was treated with one of 6 sealants and/or primer to produce seven experimental groups (15 samples/group) treated as follows: (A) no sealant or primer, (B) Leopard Light Primer, (C) SeLECT Defense (SD) Primer, (D) SD Enamel Surface Sealant, (E) Reliance Pro-Seal, (F) Ultradent OpalSeal, (G) Combined SD Primer \& Enamel Surface Sealant. The bracket-bearing blocks were exposed to mixed-organism (Streptococcus mutans and Lactobacillus acidophilus) continuous flow biofilm model (artificial mouth system) at $37^{\circ} \mathrm{C}$ for 28 days. Circulating broth was changed daily, while $10 \%$ sucrose was supplied every $6 \mathrm{~h}$ for $6 \mathrm{~min}$ to simulate meals and $\mathrm{pH}$-cycling. Teeth were harvested, and the demineralization around the brackets was measured using transverse microradiography by quantification of the mineral loss and lesion depth. No demineralization was observed either visually and quantitatively in all groups except A and B. Bonferroni protected Mann-Whitney tests showed a significant difference ( $p<0.001, \alpha=0.05)$ in both mineral loss and lesion depth between groups A and B. Antimicrobial selenium-based sealant and/or primer provided protection against enamel demineralization around orthodontic brackets comparable to resin-based sealants. Combination of Select Defense Primer and Enamel Surface Sealant produced a thicker barrier for the enamel surface.

Supported by Element34 Technology, Lubbock, Tex., USA.

\section{4}

\section{Effect of Acid Etching on the Remineralization of Artificial and Natural White Spot Lesions}

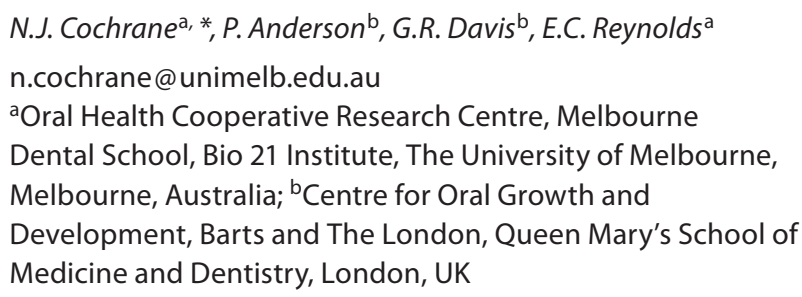

Aim: Acid etching of white spot lesions has been suggested as a means for improving remineralization. The aim of this study was to determine the effect of acid etching on the remineraliza- tion of artificial and natural white spot lesions. Experimental Approach: Six pathology-free molars and three molars with long buccal lesions were collected, photographed and sectioned to produce four and two adjacent $500 \mu \mathrm{m}$ thick sections from each respective tooth type. The artificial lesions were prepared by exposing two slices from each sound tooth to a Carbopol demineralisation buffer for 12 or 30 days. One of each of the paired sections of artificial or natural lesion was etched with a $15 \% \mathrm{HCl}$ etchant for 2 or 4-8 min respectively. The longer etching time for the natural lesions was due to their variable surface layer thickness which was determined via X-ray microtomography. The sections were mounted in a scanning microradiography system and exposed daily to a remineralization solution $(2.5 \mathrm{ml})$ for 10 days. The rate and amount of remineralization was determined by change in $\mathrm{X}$ ray absorption with time. Results: The rate of remineralization followed a linear pattern for both artificial and natural lesions. After remineralization $15 \pm 7$ and $12 \pm 4 \mu$ g hydroxyapatite was returned to the control and etched 12 day artificial lesions respectively. There was significantly less mineral returned to the etched 30 day artificial lesions $(13 \pm 6 \mu \mathrm{g})$ compared with the control (21 $\pm 4 \mu \mathrm{g})$. Etching did however improve the remineralization of the natural lesions (control: 6, 5, $6 \mu \mathrm{g}$, etched: 11, 9, $20 \mu \mathrm{g}$, respectively). Conclusion: Acid etching did not improve the response of artificial lesions to remineralization. The remineralization of natural lesions could be improved by etching however a large amount of mineral was lost in the etching process.

Supported by the Australian Dental Research Foundation Hession Award.

\section{5 \\ Remineralization Effect of Bamboo Salt on Enamel Lesion by Scanning Electron Microscopy and X-Ray Diffractometer

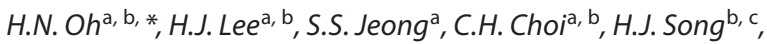 S.J. Hong ${ }^{\text {a }}$ \\ ohn326@hanmail.net; sjhong@chonnam.ac.kr \\ aDepartment of Preventive and Public Health Dentistry and \\ Dental Science Research Institute, b ${ }^{B}$ rain Korea 21 Project, and 'Department of Dental Materials, School of Dentistry, Chonnam University, Gwangju, Korea}

The aim of this study was to evaluate the remineralization effect of bamboo salt that has been used as traditional remedy for oral hygiene in Korea. Incipient artificial caries lesion was formed in bovine enamel by applying pH 5.0 lactate carbopol buffer system for $72 \mathrm{~h}$. Then, specimens were allocated into 5 groups (each group: $\mathrm{n}=4$ ), each of which has no statistical difference of Vickers hardness number. Experiment solutions include negative control, demineralization solution, $2 \%$ sodium fluoride solution $(\mathrm{NaF}$ group), 3\% bamboo salt solution (BS group), and the solution mixed with $2 \%$ sodium fluoride solution and $3 \%$ bamboo salt solution (NaF+BS group). For remineralization, the specimens were immersed in each experimental solution for $24 \mathrm{~h}$. Subsequently, the surface and cross-sectional morphologies, chemical compositions, and crystalline structure of enamels were assessed by a scanning electron microscopy (SEM) and X-ray diffractometer (XRD). SEM images showed that the enamel prisms were destructed and 
columnar small globules were deposited about $30 \mu \mathrm{m}$ thickness on the enamel surface of the NaF group. For the BS group and the $\mathrm{NaF}+\mathrm{BS}$ group, however, enamel structure was maintained and the new minerals attached on the enamel prisms were penetrated deeply over $30 \mu \mathrm{m}$ to the region of the enamel lesion. XRD patterns of the $\mathrm{NaF}$ group showed $\mathrm{CaF}_{2}$ peaks with strong intensity. This result indicates that hydroxyapatite $(\mathrm{HA})$ in enamel was substituted with $\mathrm{CaF}_{2}$. The XRD peak width of $\mathrm{HA}(002)$ in the BS group was $16.7 \%$ narrower than the control group, which means that HA crystals were more crystallized. The results of this study showed that bamboo salt may have a remineralization effect on the enamel lesion, suggesting that bamboo salt could be useful as a remineralization agent for the prevention of dental caries.

\section{6 \\ Remineralizing Effects of a CPP-ACP Containing Cream and Prolonged NaF-Toothpaste Application in situ \\ S. Paris*, T. Schellwien, C. Dörfer, H. Meyer-Lueckel \\ paris@konspar.uni-kiel.de \\ Clinic for Operative Dentistry and Periodontology, \\ Christian-Albrechts-Universität zu Kiel, Germany}

The aim of this double-blinded randomized cross-over in situ study was to compare the remineralizing effects induced by the application of either CPP-ACP containing cream (without fluoride) in addition to $\mathrm{NaF}$ toothpaste or prolonged $\mathrm{NaF}$ toothpaste application. In each of three experimental legs of four weeks, thirteen participants wore intra-oral appliances with four pre-demineralized ( $\mathrm{pH} 4.95 ; 7$ days) bovine enamel specimens $(\mathrm{n}=156)$ in the buccal flanges (flush to the acrylic). Half of each lesion was cut before the in situ phase and served for analysis of baseline demineralization. The three randomly allocated treatments were: (A) application of CPP-ACP containing cream (GC Tooth Mousse, non-fluoride) after use of $\mathrm{NaF}$ toothpaste (1,400 ppm, Lavera Zahncreme mint), (B) prolonged application of NaF toothpaste, and $(\mathrm{C})$ prolonged application of fluoride-free toothpaste (Lavera basis sensitive) serving as a negative control. Twice daily during tooth brushing participants applied either a slurry of $\mathrm{NaF}$ toothpaste $(A, B)$ or fluoride-free toothpaste $(C)$ extra-orally on the specimens for two minutes either without or with brushing (2 specimens each). After rinsing and before the appliances were inserted into the mouth again, participants applied either GC Tooth Mousse (A) NaF toothpaste slurry (B) or fluoride-free toothpaste slurry $(C)$ on the specimens, thus simulating prolonged application of toothpaste with no water rinsing immediately after brushing in groups B and C. Differences in integrated mineral loss $(\Delta \Delta \mathrm{Z})$ were calculated between values before and after the in situ period by transverse microradiography. Median (Q25/Q75) baseline mineral loss was $5,173(4,247 / 5,743)$ vol $\% \times \mu \mathrm{m}$. Treatment B induced significantly higher mineralgain $(\Delta \Delta Z)[1,307(820 / 1,994)$ vol $\% \times \mu \mathrm{m}]$ compared to treatments A $[186(-515 / 759)$ vol $\% \times \mu \mathrm{m}]$ and $C[-100(-505 / 258)$ vol\% $\times \mu \mathrm{m}]$ in both, brushed and non brushed specimens ( $p<0.05$; Wilcoxon) (data for non-brushed specimens given). In conclusion with prolonged $\mathrm{NaF}$ toothpaste application most pronounced remineralizing effects were observed.

\section{7 \\ Effect of Sucrose Concentration on Caries Lesion Formation: An in vitro Biological Caries Model}

K. Bakht ${ }^{\text {a, * , S.M. Higham }}{ }^{\text {a }}$, E. de Josselin de Jong ${ }^{\text {a, c , G.C. Martin }}{ }^{\text {, }}$, G. Burnett ${ }^{\mathrm{b}}$, C.K. Hope $\mathrm{e}^{\mathrm{a}}$

k.bakht@liverpool.ac.uk

aSchool of Dentistry, University of Liverpool, and

${ }^{b}$ GlaxoSmithKline, UK; ' Inspektor Research Systems bv, Amsterdam, The Netherlands

Frequent sucrose fermentation by plaque bacteria can cause demineralisation of dental hard tissue due to the production of organic acids. Non-biological in vitro model systems for caries do not fully represent the mix of acids produced from acidogenic species. This study aimed to investigate the effect of sucrose concentration on caries lesion formation as part of a larger endeavour to develop a biologically relevant in vitro caries model. A $200 \mu \mathrm{m}$ micrometre thick oral biofilm, representative of interproximal plaque, was produced on $5 \mathrm{~mm}$ bovine enamel discs using a constant-depth film fermenter (CDFF). The CDFF was pulsed 8 times daily for $15 \mathrm{~min}$ with $20 \mathrm{~mm}$ sucrose solution in one experiment, $100 \mathrm{~mm}$ sucrose in another and one remained unpulsed as a control. Samples were extracted at day 4, 6, 8, 10, 12 and 14 and imaged using the QLF ${ }^{\mathrm{TM}} \mathrm{D}$ before analysis to obtain $\Delta \mathrm{F}$ values as an indication of mineral content. Results from QLF $\Delta \mathrm{F}$ values revealed that pulsing with $100 \mathrm{~mm}$ sucrose provided a quicker, more pronounced caries progression pattern than pulsing with $20 \mathrm{mM}$ sucrose. At $20 \mathrm{~mm} \Delta$ F reached a maximum loss of $-17.2(\mathrm{n}=3)$ by day 14 with generally steady retention of $\Delta \mathrm{F}$ stability in preceding days. At $100 \mathrm{~mm}$ maximum loss of $-49.8(\mathrm{n}=3)$ by day 12 was observed with reduction in $\Delta \mathrm{F}$ as early as day 6 . Single factor and multi-way ANOVA tests revealed a statistically significant difference between sucrose concentrations, time and the interaction between the two factors at the $99 \%$ confidence level. In conclusion, pulsing with $100 \mathrm{~mm}$ sucrose solution induces caries more quickly than with $20 \mathrm{~mm}$ sucrose forming more pronounced demineralisation curves.

This project is funded by BBSRC GSK CASE award.

\section{8 \\ Artificial Occlusal Surface Morphology and in vitro Enamel Demineralisation}

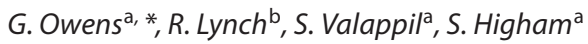

g.owens@liv.ac.uk

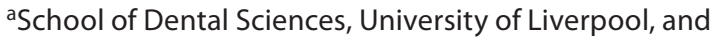

${ }^{\mathrm{b}}$ GlaxoSmithKline, Weybridge, UK

Caries lesions typically develop on the occlusal or approximal surfaces of the distal teeth. The angular nature of these sites makes plaque removal difficult aggravating the condition however these same topographic factors may also effect lesion development though altered outward diffusion of the inorganic mineral content. The aim of this work was to investigate to what extent depth and angle are able to influence the distribution of mineral loss in an artificial occlusal groove. Enamel blocks were prepared 
from the labial surfaces of bovine incisors and coated in acid-resistant varnish on five sides leaving the polished enamel surface exposed. Adjacent block pairs were then fixed at planar angles $180^{\circ} \leq \theta \geq 20^{\circ}$ and subject to demineralisation in a static acidgel system ( $1 \mathrm{~m}$ lactic acid, $\mathrm{pH} 4.6$; methylcellulose $8 \% \mathrm{w} / \mathrm{v} ; 37^{\circ} \mathrm{C}$ ). Following an incubation period of 12 days parameters of integrated mineral loss $(\Delta \mathrm{Z})$, lesion depth (LD) were measured using transverse microradiography (TMR) and considered in relation to groove depth. Average mineral loss (R) was found to correlate positively with absolute aperture $\left(R^{2}=0.8869 ; p<0.005\right)$. Moreover, lesion character was heavily influence by groove angle. Wide angles $\left(180^{\circ} \geq \theta>90^{\circ}\right)$ resulted in evenly distributed mineral loss as compared to acute angles $\left(90^{\circ} \geq \theta \geq 20^{\circ}\right)$ where both $\mathrm{LD}$ and $\Delta \mathrm{Z}$ decreased exponentially on recession from the peripheral edge. Thus, acute groove structures were less susceptible to chemical dissolution within this model. These results contrast those obtained in vivo where demineralisation is greatest at the narrowest point of the occlusal groove. Thus, the role of residual plaque in caries development is corroborated further and the need for refinement of physiologically relevant in vitro systems is emphasised.

This work forms part of a GSK funded PhD Studentship awarded to the University of Liverpool.

\section{9 \\ Remineralization Potential of a New Dental Cream and Assessment by a New Caries Detection Device \\ G. Bilgin ${ }^{\mathrm{a}, *}$, B. Korkut ${ }^{\mathrm{a}}$, F. Yanıkoğlu ${ }^{\mathrm{a}}$, B. Kargü̈l ${ }^{\mathrm{b}}$, D. Tağtekin ${ }^{\mathrm{a}}$, M. Günday a , O. Hayran', G. Stookey ${ }^{\mathrm{d}}$, B.R. Schemeron ${ }^{\mathrm{d}}$, B.D. Collins ${ }^{\mathrm{d}}$, J.S. Williams ${ }^{\mathrm{d}}$ \\ fyanikoglu@marmara.edu.tr \\ aDepartment of Restorative Dentistry, Marmara University, \\ ${ }^{b}$ Department of Pedodontics, Marmara University, and \\ 'Department of Health Science, Yeditepe University, Istanbul, \\ Turkey; ${ }^{\mathrm{d}}$ Therametric Technologies, Inc., Noblesville, Ind., USA}

The aim of this study was to evaluate the effectiveness of a new water based cream that contains hydroxyapatite and fluoride on remineralization of white spot enamel lesion and assessment by a new detection device, FluoreCam. Demineralized human enamel specimens were measured for baseline QLF, Ultrasound, Diagnodent and FluoreCam. Ten specimens in each of four groups were used in this in vitro recycling study with the following treatments: (1) CPP-ACP agent (GC Tooth Mousse) three times daily, (2) hydroxyapatite and fluoride agent (Remin Pro) three times daily, (3) Bifluoride Varnish once a week, (4) sodium fluoride (IpanaKalsiDent) toothpaste three times daily. The recycling demin-remin and treatment regimens were continued for 21 days. The posttreatment data were obtained by measurements of QLF, Ultrasound, Diagnodent and FluoreCam. Statistical analyses of the data included Chi-square test (DIAGNOdent peak values), Kolmogorov-Smirnov test (ultrasound), and an ANOVA test with Tukey's HSD test (fluorescence \& microhardness). Significant differences between treatments were observed by fluorescence assessments but not by ultrasound. Delta Q data (FluoreCam) indicated the sodium fluoride group provided significantly less re- mineralization than the other three treatments. While the other three treatments did not differ significantly treatment 2 was numerically a little less effective than treatments 1 and 3 although all 4 treatments resulted in some remineralization.

We are very thankful to Prof. Aysen Yarat and Dr. Sevker, to Eng. Günay Ozkas from Cevre Chemistry, Esra Ozsoy from Birpa Chemistry for their valuable helps for the laboratory materials, and also to the Assistants of the Department of Restorative Dentistry of Marmara University and to the Staff of NDT Service of Turkish Airlines.

\section{0 \\ Influence of Matrix Metalloproteinase 2 (MMP-2) on Caries-Odontoblast Reaction and Formation of Reactionary Dentine}

\author{
N. Charadram*, N. Hunter, M. Swain \\ ncha1431@uni.sydney.edu.au \\ Faculty of Dentistry, The University of Sydney, Australia
}

Reactionary dentine is a protective matrix formed by odontoblasts in response to caries-associated invading bacteria. The anisotropic structure of reactionary dentine is considered to limit bacterial invasion. Dentine sialophosphoprotein (DSPP) is substantially up-regulated as a driver of this altered matrix. DSPP is secreted as a large protein which is functionally processed by activated MMP-2, also secreted by odontoblasts. Therefore, the synthesis and activation of MMP-2 are critical determinants of reactionary dentine. The aim of this study was to clarify the factors that control MMP-2 expression and activation in carious lesions. Twenty two molar teeth with cavitated carious lesions limited to invasion of less than half the depth of dentine and ten healthy teeth were used for the study. RNA from the odontoblastic layer was extracted to investigate the levels of messenger RNA (mRNA) for nominated products. MMP-2 was detected by immunohistochemistry (IHC). The other half of the tooth was used for $\mathrm{pH}$ measurement and specific gelatinase assay reflecting MMP-2 activity. MMP-2 expression was up-regulated by odontoblasts during carious disease and also TIMP-2. However, MT1-MMP, an MMP-2 specific activator, was found to be markedly increased leading to the activation of MMP-2. Confirmed by MMP-2 activity that was higher in sound dentine beneath the lesion. IHC also clearly established that MMP-2 is present in that layer while $\mathrm{pH}$ measurements demonstrated that acidity was not implicated in activation of MMP-2 in that layer. During the progression of carious lesions, odontoblasts respond by secreting more DSPP to facilitate the protective barrier of reactionary dentine. In a coordinated response odontoblasts secrete and activate MMP-2 to process DSPP. The process represents a transition from the precision of physiological dentine to the planned disorder of reactionary dentine.

The authors thank Dr. Derek Harty, Dr. Catherine Rathsam and staff in the Institute of Dental Research (IDR) and Biomaterials, Faculty of Dentistry for help and informative discussions. Naresuan University Thailand Staff Development Project scholarship (for N.C.). Australian Dental Research Foundation Inc. (ADRF) for a grant. 


\section{1}

Microstructure of Dental Enamel after the Combined Effect of $\mathrm{HCl}$ Gel Etching and Toothbrushing

A. Kiesow*, M. Morawietz, A. Cismak, S. Sarembe, M. Petzold

andreas.kiesow@iwmh.fraunhofer.de

Fraunhofer Institute for Mechanics of Materials, Halle (Saale), Germany

Currently developed micro-invasive caries treatments using resin infiltration techniques involve an etch gel pre-treatment that inevitably leads also to etching of sound enamel adjacent to the lesion. The aim of this study was to investigate the effect of the etch gel treatment with respect to potential surface damage and the effect of following toothpaste brushing on the treated areas. Specimens consisted of both natural and polished human enamel samples prepared from unerupted third molars. The samples were etched using a $15 \% \mathrm{HCl}$ etch gel (Icon-Etch $\left.{ }^{\circledR}\right)$ while for brushing an automated brushing machine (load $2 \mathrm{~N}$ ) operating in toothpaste slurry (two parts water/one part toothpaste
(Elmex ${ }^{\circledR}$ Kariesschutz)) was used. The study design contained five groups for both natural and polished specimens: (i) untreated, (ii) untreated plus brushing with 10,000 cycles, (iii) etched, (iv) etched plus brushing with 1,000 cycles, and (v) etched plus brushing with 10,000 cycles. After treatment, the enamel samples were analysed using surface profilometry and scanning electron microscopy (SEM) in both surface and in cross-section. Profilometry revealed a roughness increase due to the treatments with the following ranking in terms of average roughness data $\left(\mathrm{R}^{\mathrm{a}}\right.$ : (i) < (ii) < (iii) < (iv), (v)). For etching only (iii), the crosssection SEM investigation showed demineralised regions with depth of about two microns. For both polished and natural surfaces, these demineralised zones gradually diminished after 1,000 and disappeared almost completely after 10,000 brushing cycles without additional defect formation. As only a demineralised surface zone of low thickness is polished away by toothpaste brushing it can be concluded that a single $\mathrm{HCl}$ etch gel treatment does not leave significant damage or defects in the remaining enamel.

The support of DMG Dental Materials GmbH, Germany is gratefully acknowledged. 


\section{Caries Research}

\section{Session 4 Epidemiology}

\begin{abstract}
62
Familiarity of Pregnant Women Relating Caries Prevention Methods

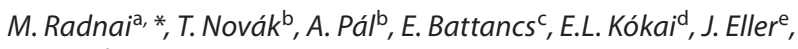
1. Gorzóf

martaradnai@yahoo.com

Departments of aProsthetic Dentistry and Oral Biology, Faculty

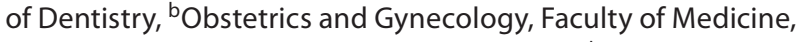
'Conservative Dentistry, Faculty of Dentistry, ${ }^{\text {dPedodontics }}$ and Orthodontics, Faculty of Dentistry, ${ }^{e}$ Medical Physics and Medical Informatics, Faculty of Medicine, and fPeriodontology, Faculty of Dentistry, University of Szeged, Hungary
\end{abstract}

The aim of the study was to collect data from pregnant women about their knowledge of caries preventive methods and those they planned to use for their children. The aim was also to assess how and if this information was associated with the age, place of residence, educational level and occupation of the mother. A questionnaire was prepared and used in the survey. Questions related to the knowledge of fluoridation, diet of children, consumption of sweets and acidic drinks and tooth brushing of children. The pregnant women $(n=500)$ answered the questions voluntarily at the university clinic of Szeged/Hungary. Standard statistical methods were used. The mean age of the women was 30.7 years. Most of the women were city dwellers; they were of different educational levels and occupations. They stated that they had read about caries preventive methods during pregnancy $(95.5 \%)$ and many of them (75.6\%) thought they were familiar with them. They planned to start to brush the baby's teeth mostly $(70.5 \%)$ from 1 year of age. One third of the women thought that children were able to brush their teeth alone at the age of six years. Women with a higher education and working as professionals had a little more knowledge about preventive methods, but this difference for most of the answers was not significant. In conclusion, there were no great differences relating to the preventive knowledge and the socio-demographic factors. This shows that the nec- essary information reached the women irrespective of their residence, educational level or occupation. Health staff have to distribute this knowledge and make sure it is being adhered to, since $\mathrm{dmft}$ scores in Hungary are worse than in many European countries.

\author{
63 \\ Urban-Rural Distribution in Dental Caries among \\ 4- to 5-Year-Olds in Arkhangelsk Region, Northwest \\ Russia \\ M. Pastbin ${ }^{\text {a, b, * , M. Gorbatova }}$ a, b, I. Pastbina ${ }^{\text {c }}$ L. Gorbatova ${ }^{\text {b }}$ \\ mikpastbin@gmail.com \\ anternational School of Public Health and bepartment of \\ Pediatric Dentistry, Northern State Medical University, and \\ 'Ministry of Health and Social Development of the Arkhangelsk \\ Region, Arkhangelsk, Russia
}

The aim of this study was to investigate the regional urbanrural distribution of dental caries among 4- to 5-year-olds in Arkhangelsk region, Northwest Russia. In 2010, a cross-sectional survey was conducted in two (out of 8) randomly selected kindergartens in Mirny town and in all (two) kindergartens in Emetsk village. The total number of children examined was 131 (53\% boys) with mean age of 4.5 (SD 0.5) years. The caries experience status of primary teeth was assessed by a calibrated dentist in accordance with the WHO 1997 criteria at the d3 level and $\mathrm{d}(3) \mathrm{mft} / \mathrm{d}(3) \mathrm{mfs}$ values (presented as mean \pm standard error); Care Index $(\mathrm{ft} / \mathrm{d}(3) \mathrm{mft} \times 100)$ was calculated. The children were divided into two groups: urban $(n=89)$ and rural $(n=42)$, respectively. Chi-squared tests and Mann-Whitney tests were used for the dichotomous and numerical data, respectively. The study was approved by the Ethical Committee of the Northern State Medical 
University, Arkhangelsk, Russia. Altogether, 61 (68\%) (95\% CI: $58-77)$ of the urban children and 37 (88\%) (95\% CI: 75-94) of the rural children had dental caries $\left(\chi^{2}=5.791, \mathrm{p}=0.02\right)$. The corresponding mean $\mathrm{d}(3) \mathrm{mft} / \mathrm{d}(3) \mathrm{mfs}$ values were $4.35 \pm 0.48 / 8.51 \pm$ 1.15 and $8.00 \pm 0.70 / 18.24 \pm 2.15(\mathrm{p}<0.001)$. There was a significant difference in $\mathrm{dt} / \mathrm{ds}$ component $3.75 \pm 0.43 / 6.88 \pm 0.97$ and $6.81 \pm 0.63 / 15.10 \pm 1.88(\mathrm{p}<0.001)$ respectively. There was a significant increase in the Care Index with age in $4.1 \%$ for 4 -year-olds and $15.1 \%$ for 5 -year-olds $(p=0.005)$ among urban children, while in the rural area there was an observed decline from 13.7 to $9.5 \%$, respectively $(\mathrm{p}=0.725)$. No significant differences were observed between 4 -year-olds $(p=0.094)$ and 5 -yearolds $(p=0.220)$, respectively. There were no gender differences within and between districts. In the groups studied, children in the urban area appeared to have less caries and a higher proportion of filled teeth in comparison with those living in the selected rural area. Overall dental caries levels in Arkhangelsk region are still high and special preventive measures are needed to improve the situation.

Personal funds were used for the implementation of this study.

\section{4 \\ Comparison of the Development in Caries Prevalence in Mecklenburg-Vorpommern and Germany from 1991 to 2010 \\ C.H. Splieth* \\ splieth@uni-greifswald.de \\ Department of Preventive and Pediatric Dentistry, \\ Ernst-Moritz-Arndt University, Greifswald, Germany}

After the unification of Germany, caries prevalence was highest in the North and East of Germany, especially in the state of Mecklenburg-Vorpommern (MV). Thus, the aim of this study was to analyse the caries development in MV from 1991 to 2010 in 6-7- and 12-year-olds in comparison to the German national surveys. The data were extracted from the results of the compulsory, dental examinations in kindergartens and schools in the 16 cities and districts in MV including about 10,000 6- and 12-yearolds each year and compared to a representative East German survey [Institut der Deutschen Zahnärzte, 1991] and further representative cohorts, also examined in school (6-7- and 12-yearolds) [Deutsche Arbeitsgemeinschaft Jugendzahnpflege, 2010]. Caries in 12-year-olds decreased in MV continuously from a high of 3.8 DMFT (1991) to currently 0.87. The DAJ samples for MV and the full cohorts for the same years showed very similar caries prevalence. The mean DMFT figures for MV were always higher than the German mean, increasing in the $90 \mathrm{~s}$ from 115\% (3.8 DMFT) to a maximum of $163 \%$ (2.2 DMFT; 2000) of the national level and decreasing to $136 \%$ (0.9 DMFT) in 2009. The caries prevalence in 6-7-year-olds decreased to a lesser extent. In the full cohort of 6-year-olds, caries prevalence, as $\mathrm{dmft}$, decreased from 3 to 1.7. The samples of 6-7-year-olds in the DAJ studies exhibited considerably higher $\mathrm{dmft}$ figures ranging from $138 \%$ (4 dmft) to $119 \%$ (2.6 dmft) of the national German level, where the caries reductions were also less pronounced. Thus, caries prevention was especially successful in adolescents in MV and more attention should be attributed to the primary dentition.

Thanks to the cooperation by the Ministry of Social Affairs, Mecklenburg-Vorpommern, Germany.

\section{5 \\ Impact of Dental Caries Experience on Oral Health-Related Quality of Life of Brazilian Preschoolers and Families}

A.C. Scarpelli, , I.A. Pordeus ${ }^{\text {a }}$, F.M. Ferreira ${ }^{\text {b }, ~ C . M . ~ V i e g a s ~}{ }^{\text {a }}$, A.C. Carvalho a, S.M. Paiva ${ }^{\mathrm{a}, *}$

smpaiva@uol.com.br

aDepartment of Paediatric Dentistry and Orthodontics, Federal University of Minas Gerais, and bepartment of

Paediatric Dentistry, Federal University of Paraná, Brazil

The aim of this study was assess the impact of dental caries experience on each item of a child's Oral Health-Related Quality of Life (OHRQoL) instrument. A population-based cross-sectional survey was carried out with 1,632 parents/caregivers and their 5 -year-old children. Children were randomly selected, using twostage cluster sampling, from public and private preschools in Belo Horizonte, Brazil. A single general dentist previously calibrated $(\mathrm{k}=0.96)$ performed dental examinations of the children following the criteria standardized by the World Health Organization. Caregivers self-completed the Brazilian Early Childhood Oral Health Impact Scale (B-ECOHIS) and a form addressing sociodemographic status. Caries experience $(\mathrm{dmft} \geq 1)$ was included in models of multiple Poisson regression analysis, with robust variance estimation, with the purpose of investigating in which item of the B-ECOHIS the impact was present. The models were adjusted by socio-demographic (child's position, type of preschool, caregiver's age and family income) and clinic (traumatic dental injury, malocclusion and developmental enamel defects) covariables associated with the impact of oral condition on OHRQoL in univariate models. The prevalence of reported impact of oral condition on the child and family's OHRQoL were 35.9 and $30.9 \%$ respectively. Dental caries experience was significantly associated with all items of B-ECOHIS. Children with caries experience had 'missed preschool' with a frequency 12.2 times greater $(95 \% \mathrm{CI}$ $6.2-24.4)$ than those caries-free. The main items in family section with impact were 'taken time off from work' (PR 5.2; 95\% CI 4.46.1 ) and 'had a financial impact of child dental treatments' (PR 5.0; 95\% CI 3.3-7.7). According to parents' perceptions, OHRQoL was negatively and consistently impacted by dental caries experience, mainly on functional domains.

This study was supported by FAPEMIG and CNPq. 


\section{6}

\section{Caries Pattern in Preschool Children and Related Background Factors}

G. Congiu $^{\mathrm{a}, *}$, E. Maiorini ${ }^{\mathrm{b}}$, G. Spano ${ }^{\mathrm{a}}$, P.F. Lugliè ${ }^{\mathrm{a}}$, G. Campus ${ }^{\mathrm{a}}$

giannicaro83@yahoo.it; gcampus@uniss.it

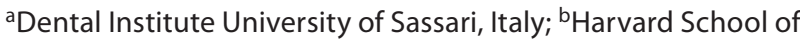

Dental Medicine, Boston, Mass., USA

The purpose of this study was to evaluate the prevalence, severity and background variables associated with early childhood caries (ECC) in preschool children (aged 2-4), attending public nursing schools in the town of Sassari (Italy). Subjects were selected using a systematic cluster methodology. Caries status was determined at D1-D3 level by one calibrated clinical examiner, using standardized conditions. Information about the socio-economic status of the family, background health of the children (pre-term birth, chronic diseases), diet regimens, oral hygiene habits (toothbrushing frequency, fluoride consumption) was collected through standardized questionnaires filled in by the parents. Data analysis was performed using Stata $10^{\circledR}$ software. 373 (192 males, 181 females) were examined. Caries experience (D1-D3) was observed in $28.69 \%$ (95\% CI $=24.65-32.71 \%)$ of children. Gender was not significantly associated with caries experience. Caries experience was significantly associated with educational level of parents $(\mathrm{p}<$ $0.01)$, use of pacifier $(\mathrm{p}=0.04)$, sweet-drink before bedtime ( $\mathrm{p}=$ $0.04)$, mean numbers of meals/day $(\mathrm{p}=0.04)$. Two different approaches were used for the multivariate analysis: stepwise logistic regression model was run using caries experience as dependent variable and a zero inflated regression model (ZIP) was run. In logistic regression the schooling level of the parents play a protective rule regarding caries experience $(\mathrm{OR}=0.80,95 \% \mathrm{CI}=0.72-0.90)$, while children fed sweet drinks before bedtime had a higher risk to developed caries $(\mathrm{OR}=1.76,95 \% \mathrm{CI}=1.01-3.09)$. The output of the ZIP model showed that the probability to have an extra zero (to have no caries) is linked only to the schooling level of the parents $(\mathrm{p}=0.01)$. In conclusion, ECC in children living in Sassari was strongly associated with consumption of sweet drinks before bedtime, family- and child-care related factors and tooth-brushing.

\section{7}

Impact of Caries and the Consequences of Untreated Cavitated Lesions on Brazilian Children's Quality of Life

S.C. Leal ${ }^{\mathrm{a}}$, E.M. Bronkhorst ${ }^{\mathrm{b}}$, J.E. Frencken ${ }^{\mathrm{b}, \mathrm{c}, *}$

sorayaodt@yahoo.com

${ }^{a}$ Department of Dentistry, Faculty of Health Sciences, University of Brasília, Brazil; Departments of ${ }^{b}$ Preventive and Restorative Dentistry and ' $\mathrm{Global}$ Oral Health, College of Dental Sciences, Radboud University Nijmegen Medical Centre, The Netherlands

The aim was to investigate the impact of caries prevalence and the consequences on untreated cavitated dentine lesions on the quality of life of Brazilian schoolchildren. Three calibrated examiners assessed 835 children aged 6 and 7 years. The ICDAS II was used to determine caries prevalence and the prevalence of pulp exposure, ulceration, fistula and abscess in relation to dental caries was scored through pufa, an index developed to register the consequences of untreated cavitated dentine lesions. History of extraction and toothache were recorded. Oral health-related quality of life was assessed using the Early Childhood Oral Health Impact Scale (ECOHIS) that is comprised of 13 items - 9 related to the child and 4 to the family. Independent $t$ tests were used to analyse the relationship between the variables: caries prevalence, pufa ( 0 or $\geq 1$ ), history of extraction (yes/no) and toothache (yes/no) with the ECOHIS scores. 587 questionnaires were returned fully replied and analysed. The prevalence of cavitated dentine lesions and that of pufa were 75.1 and $26.2 \%$, respectively. Toothache was reported by $22 \%$ of the children and $9.2 \%$ of them had been submitted to a tooth extraction. The ECOHIS scores were significantly higher $(\mathrm{p}<0.001)$ in children with caries-related variable scores $\geq 1$. Children without cavitated dentine lesions had significantly lower chance on a high ECOHIS score (OR $=0.46$, CI: 0.29-0.72) than those without such lesions. Children with pufa $\geq 1$ (OR $=$ 6.37, CI: 2.87-4.14), history of extraction (OR = 8.6, CI: $1.97-37.51)$ and toothache $(\mathrm{OR}=6.16, \mathrm{CI}: 2.51-15.11)$ had significantly higher chance on a high ECOHIS score than those not having these conditions. It can be concluded that children's quality of life was negatively influenced by the presence of cavitated dentine lesions, pufa scores $\geq 1$, toothache and tooth extraction.

This study was supported by FAP-DF (Fundação de Apoio à Pesquisa do Distrito Federal).

\section{8 \\ Individualized Caries Control of 0- to 3-Year-Olds in Greenland: Strategy and Results \\ F. Senderovitz ${ }^{\mathrm{a}}$, K.R. Ekstrand ${ }^{\mathrm{b}}{ }^{\text {, J. Christiansen }}{ }^{\mathrm{c}, *}$, M.E.C. Christiansen ${ }^{c}$, R.M. Santamaria ${ }^{\mathrm{d}}$ \\ jettech@mail.dk \\ ${ }^{a}$ Chief Dental Officer, National Management of Health, Greenland; b Department of Cariology and Endodontics, Dental Faculty, University of Copenhagen, Copenhagen, and ${ }^{\mathrm{C}}$ Former Chief Dental Officers, Child Dental Health Service, Municipality of Nexö, Denmark; ${ }^{d}$ Department of Preventive and Paediatric Dentistry, University of Greifswald, Greifswald, Germany}

Since the 1980's the Public Dental Health Service in Greenland has offered free dental health care to preschool- and school-children based on the principles of the Child Oral Health System in Denmark. However, Greenland still has a very high caries prevalence. Therefore a new strategy focusing on individualized caries control for all children was implemented in 2008. The aim of this study was to describe the strategy for 0 - to 3 -year-olds and to describe the caries status of 3 -year-olds in 2010 the majority of whom had followed the new strategy and to compare these results with data of 3-year-olds from previous years. Parents and children attended clinics when the children were 8,14 and 26 months of age. At each visit local dental hygienists/assistants educated and trained parents in brushing the teeth of their child using 1,450 ppm F toothpaste. def-s was recorded using the criteria of the Danish Child Oral Health Service: $d$ = active caries with cavitation, $\mathrm{e}=$ extracted due to caries, $\mathrm{f}=$ filled. Sample A: 3 -year-olds 
in 2008 ( $\mathrm{n}=397)$, sample B: 3-year-olds in $2009(\mathrm{n}=447)$, and sample C: 3 -year-olds in $2010(n=515)$; drawn from total cohorts of 846,814 and 813 , respectively. From group A and B the national dental health data of Greenland were used, data from group $\mathrm{C}$ were computerized. def-s $=0$ in sample A was $64 \%, \mathrm{~B}=64 \%$ and $\mathrm{C}=73 \%$, the mean (SD) def-s was 2.82 (6.82), 3.61 (8.26) and 2.05 (5.68). Unpaired t test showed significant difference in mean defs between sample B and C $(\mathrm{p}<0.007)$, less difference between sample $A$ and $C(p=0.07)$, no significant difference between sample $A$ and $B(p=0.13)$. In conclusion, the prevalence of caries in group $\mathrm{C}$ was less compared to group A and significantly less compared to group $B$.

The work done by the personal working in the Child Dental Health Service in Greenland is highly acknowledged.

\section{9}

\section{Prevalence and Distribution Pattern of Caries in Greenlandic 3-Year-Olds following a Strategy Based on Individualized Caries Control}

\author{
F. Senderovitz a , K.R. Ekstrand ${ }^{\mathrm{b}}$, J. Christiansen", \\ M.E.C. Christiansen', R.M. Santamaria ${ }^{\text {, * }}$ \\ ruth.santamaria@stud.uni-greifswald.de \\ ${ }^{a}$ Chief Dental Officer, National Management of Health, \\ Greenland; b Department of Cariology and Endodontics, Dental \\ Faculty, University of Copenhagen, Copenhagen, and 'Former \\ Chief Dental Officers, Child Dental Health Service, Municipality \\ of Nexö, Denmark; ${ }^{\mathrm{d} D e p a r t m e n t}$ of Preventive and Paediatric \\ Dentistry, University of Greifswald, Greifswald, Germany
}

To reduce the very high caries prevalence in Greenland, a new caries strategy focusing on individualized caries control for all children in Greenland was implemented in 2008. The strategy among other things involved parents to bring their children to the dental clinics when they became 8, 14, 26 and 36 months of age. The aim of this study was to assess the prevalence and distribution of dental caries on 3-year-olds in Greenland, whom from they were 8 months to the age of about 3 were offered the new strategy. A total of 5153 -year-olds (64\% of the total children population) were examined by calibrated ( $>75 \%$ agreement) dental hygienists/ dentists in the 17 districts in Greenland. The Danish caries criteria were used for the caries assessment $(1=$ cavitated caries, $2=$ secondary caries, $4=$ restoration, $6=$ extracted due to caries). The children's files were accessed to assess their compliance with the new strategy, so that comparisons between compliant and noncompliant individuals could be performed. Statistical significance was set at a $p$ value $<0.05$. The mean age at the time of examination was 35 months $(\mathrm{SD}=4.8)$. For the whole group of children the def-s $=0$ was $73 \%$; the mean def-s was 2.05 ( $S D=5.68)$; the d-component corresponded to $>85 \%$ of the index. No significant difference $(p>0.05)$ between contralateral surfaces in the same arch were found. The surfaces most affected by caries were: the mesial (15\%) and distal (9\%) surfaces of 51-61 and the occlusal surface $(7 \%)$ of $74-84$. The def-s inter-district variation was between 0.28 and 12.2. Data from the patient files disclosed that the new strategy was not fully implemented in three of the 17 districts. In these districts the mean def-s were significantly higher than in the districts were the children had followed the strategy $(\mathrm{p}<0.05)$. At surface level significant differences were seen at the mesial surfaces of 51, 52, 62 and at the occlusal surfaces of 65,74 , $84(p<0.05)$. In conclusion, the prevalence of caries in Greenland varies between districts; the effort in following the new strategy differs between districts. Those districts having followed the new strategy had in general lower caries prevalence. The caries distribution within the dentition in districts which have followed the strategy versus those which have not, were the same, but at a higher level on each surface in the latter districts.

The work done by the personal in the Child Dental Health Service in Greenland is highly acknowledged.

\section{0 \\ Caries Prevalence in Young Children and Parents' Compliance with Preventive Programs \\ E.E. Maslak ${ }^{\mathrm{a}, *}$, N.V. Kuyumdjidi ${ }^{\mathrm{a}}$, V.V. Shkarin ${ }^{\mathrm{b}}$, M.L. Yanovskaya ${ }^{\mathrm{b}}$, D.S. Vlasova ${ }^{a}$, A.S. Rodionova ${ }^{a}$ \\ eemaslak@yandex.ru \\ ${ }^{a}$ Children's Dentistry Department, Volgograd State Medical \\ University, and bealth Care Department of Volgograd \\ Administration, Russia}

The aim of our research was to study caries prevalence in 1- to 3 -year-old children and parents' compliance with the caries preventive program. 389 children (155 1-year-olds, 119 2-year-olds, 1153 -year-olds) were enrolled in the investigation. The children from different districts of Volgograd were examined by a trained and calibrated dentist: 1-2-year-olds during their routine visits to pediatric clinics (4 clinics), 3-year-olds during their kindergarten time (6 kindergartens); $\mathrm{d}_{3-4} \mathrm{mft}$ index was used to score caries (mean $\pm \mathrm{SE}$ ). Informed parents' consents were obtained and all parents were informed about the caries preventive program for young children, received instructions on oral hygiene, counseling on diet and fluoride consumption, recommendations on dental office attendance for professional prevention and treatment according to the child's oral health and caries risk. After 2 years the parents' compliance with the preventive program was assessed through 336 mothers' interviewing. 336 (86.37\%) children were re-examined. The analysis was performed using the SPSS 10.0. The results: at baseline caries free children were $69.31 \%$ (1-yearolds $91.61 \%$, 2 -year-olds $68.07 \%$, 3 -year-olds $40.87 \%$ ), $\mathrm{d}_{3-4} \mathrm{mft}$ index was $1.39 \pm 0.23$ (1-year-olds $0.35 \pm 0.09$, 2-year-olds $1.36 \pm$ 0.20 , 3 -year-olds $2.85 \pm 0.45$ ). After 2 years only $26.19 \%$ children were caries free, $19.05 \%$ had all caries lesions filled, and $54.76 \%$ had both filled and untreated caries lesions; $\mathrm{d}_{3-4} \mathrm{mft}$ was $3.96 \pm$ 0.69 (2.67 $\pm 0.38,4.09 \pm 0.56$, and $5.48 \pm 0.89$ according to the age groups mentioned above), the differences between $\mathrm{d}_{3-4} \mathrm{mft}$ at baseline and at the end of the study were significant for all age groups, $\mathrm{p}<0.05$. Caries preventive recommendations on oral hygiene for children were provided in $36.91 \%$ families, on diet in $32.14 \%$, on fluoride consumption in $41.07 \%$, on dental clinic attendance for professional prevention (fluoride varnish, fissure sealant) in $45.24 \%$ ones. In conclusion, caries prevalence in young children was high and increased with age; the level of parents' 
compliance with the caries preventive program was low; the caries preventive program should start earlier, in pregnancy, and be continued during the first and the further years of childhood.

The authors would like to acknowledge personally T.K. Berdikova and V.E. Lomovskih for their help and the heads of pediatric clinics and kindergartens for cooperation and help. Sources of funding: Volgograd State Medical University.

\section{1}

\section{Dental Caries in Adult People in Belarus}

N. Yudina*, A. Rusak, O. Yuris, D. Brovka, D. Shabunko

rusak@mol.by

Belarusian Medical Academy of Post-Graduate Education,

Belarus

Long-term monitoring of dental caries prevalence in Belarus demonstrated an increase in all age groups in 1996. The national program for prevention of dental caries was introduced in 1998. This program included caries preventive recommendations for citizens of Belarus including brushing teeth with toothpaste containing fluorides (1,000-1,500 ppm), using salt containing fluorides $(250 \mathrm{mg} / \mathrm{kg})$ for cooking and taking food no more than 5 times per day. The aim of this study, which was performed from July to October 2010, was to determine efficiency of the national program for dental caries prevention in adult. Four calibrated dentists evaluated dental status of 1,531 people in different region of Belarus. The DMFT was recorded in subjects aged 18 years (males 328, females 446) and in subjects aged 35-44 years (males 319, females 438). Criteria for DMFT: D - caries and filling with decay, $\mathrm{M}$ - missing teeth, $\mathrm{F}$ - filling and crown (excluded bridge abutment). The mean ( \pm SE) DMFT in the 18-year-old subjects was $5.71( \pm 0.14), \mathrm{D}$ was $1.71( \pm 0.09), \mathrm{M}$ was $3.65( \pm 0.11)$ and $\mathrm{F}$ was $0.26( \pm 0.02)$ while in 1996 DMFT in this age group was 6.8 . In the 35- to 44-year-old group the mean DMFT was $11.93( \pm 0.18)$ with D $1.71( \pm 0.09)$, M $7.57( \pm 0.16)$ and F 2.65 ( \pm 0.1$)$ compared to the 1996 mean DMFT score of 13.8. In conclusion these results suggest that the preventive program was effective in reducing dental caries in Belarus and confirm the efficiency of the national caries preventive program.

\section{2 \\ Decline of Dental Caries by Community Caries Prevention Program in a Suburban Area}

J.B. Kim ${ }^{\mathrm{a}}$ * , D.H. Han ${ }^{\mathrm{b}}$, S.M. Lee ${ }^{\mathrm{c}}$, M.J. Kim ${ }^{\mathrm{a}}$, E.J. Jun ${ }^{\mathrm{a}}$

jbomkim@pusan.ac.kr

aSchool of Dentistry, Pusan National University, Yangsan,

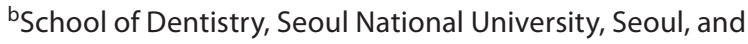

'Department of Dental Hygiene, Kyungnam College University

of Information and Technology, Busan, South Korea

The purpose of this study was to confirm the decline of dental caries among children benefited from the community caries prevention program composed of 13-year sealant and 9-year water fluoridation program implemented by the public health center in Hapcheon county, Korea. The permanent teeth of children ( $\mathrm{n}=$ 1,528 ) aged 6 to 14 years in Hapcheon county were examined in 2009 year by two calibrated dentists who took part in the Korean national dental survey of 2006. All children in Hapcheon county were benefited from free sealants on molars and premolars and water fluoridation program. Caries reduction was estimated by the comparison of DMFT scores among study children with the 2006 Korean national data of suburban areas. Over $90 \%$ of children in Korea are estimated to use fluoride-containing toothpastes. Caries prevalence rates were 15.9, 33.2 and $42.5 \%$ among 8-, 10 - and 12-year-olds in Hapcheon county, respectively; 30.3, 42.0 and $68.7 \%$ among same aged Korean national suburban children, respectively. DMFT scores were $0.26,0.65$ and 1.07 among 8-, 10- and 12-year-olds in Hapcheon county and 0.63, 0.82 and 2.60 among same aged suburban children in the 2006 Korean national survey. Differences of DMFT scores were $0.41,0.17$ and 1.53 among 8-, 10- and 12-year-olds between Hapcheon county and Korean national suburban area. Caries reduction rate of children aged 12 years was estimated to be $58.8 \%$. In conclusion, dental caries among children in Hapcheon county declined by the community caries prevention program composed of sealant and water fluoridation program implemented by the public health center in Hapcheon county, Korea.

This work was supported by the Public Health Center in Hapcheon County, Korea.

\section{3 \\ Reductions in Dental Caries in Three-Year-Old Children in Greater Glasgow and Clyde}
A.D. McMahon ${ }^{\mathrm{a}, *}$, Y. Blair ${ }^{\mathrm{b}}$, D.R. McCall ${ }^{\mathrm{b}}$, L.M.D. Macpherson ${ }^{\mathrm{a}}$
alex.mcmahon@glasgow.ac.uk
${ }^{a}$ Community Oral Health, Glasgow Dental School, School of Medicine, College of Medicine, Veterinary and Life Sciences, University of Glasgow, and ${ }^{\mathrm{b}}$ Oral Health Directorate, NHS
Greater Glasgow and Clyde, Scotland

The objective of this study was to examine dental inspection data from three-year-old children in Greater Glasgow and Clyde, Scotland, over four years to assess oral health trends during a period of implementation of a national child dental health improvement programme (Childsmile). Dental inspections of three-yearold children in Greater Glasgow and Clyde were undertaken in the academic years 2006/7, 2007/8 (the baseline years), 2008/9 and 2009/10 (post intervention). The number of decayed, missing and filled teeth was calculated $(\mathrm{d} 3 \mathrm{mft})$, together with the percentage $\mathrm{d} 3 \mathrm{mft}>0$. The study also examined the effect of socioeconomic status using the Scottish Index of Multiple Deprivation (SIMD). In total, 10,022 children were inspected (19\% of the population). The weighted percentage of children with caries experience was $26 \%$ in $2006 / 7,25 \%$ (2007/8), reducing to $18 \%$ (2007/8) and $17 \%$ $(2009 / 10)$. When compared to the first baseline year of $2006 / 7$, the OR was 0.91 for $2007 / 8(0.79-1.06, \mathrm{p}=0.221), 0.63$ for $2008 / 9$ $(0.55-0.72, \mathrm{p}<0.001)$, and 0.50 for $2009 / 10(0.43-0.58, \mathrm{p}<0.001)$. The weighted mean d3mft was 1.1 in 2006/7, 1.0 in $2007 / 8$ (p = $0.869), 0.6$ in 2008/9 ( $<<0.001)$ and 0.4 in $2009 / 10$ ( $p<0.001)$. 
Caries reductions were seen in all socioeconomic groups. This study demonstrates that it is possible to impact upon the prevalence and morbidity of dental caries across the socioeconomic spectrum in a population. The dental health of young children in the NHS Greater Glasgow and Clyde Board area has improved significantly in recent years. Clyde.

The data collection was funded by NHS Greater Glasgow and

\section{4 \\ Measures of Health Inequality Applied to Dental Inspection Data}

\section{A.D. McMahon a, Y. Blair ${ }^{\text {b, * , D.R. McCall }}{ }^{\text {, L.M.D. Macpherson }}{ }^{\mathrm{a}}$ \\ alex.mcmahon@glasgow.ac.uk \\ ${ }^{a}$ Community Oral Health, Glasgow Dental School, School of Medicine, College of Medicine, Veterinary and Life Sciences, University of Glasgow, and boral Health Directorate, NHS \\ Greater Glasgow and Clyde, Scotland}

The aim of this study was to apply tests of health inequality to dental inspection data from three-year-old children in Greater Glasgow and Clyde and to compare and contrast the usefulness of these techniques. Dental inspections were undertaken in the academic years of 2006/7, 2007/8, 2008/9 and 2009/10. The measures of caries experience used were $\mathrm{d}_{3} \mathrm{mft}>0$ and mean $\mathrm{dmft}$, while the measure of socioeconomic status was 'quintiles' of the Scottish Index of Multiple Deprivation (SIMD). Analyses of each year's data included the following: raw summaries by SIMD score; logistic regression (LR) models with ROC plots and c-index; Significant Caries Index (SIC); Population Attributable Risk (PAR); Concentration Index; Gini Coefficient and Lorenz Curves; Slope Index of Inequality (SII) and Relative Index of Inequality (RII). We inspected 10022 three-year-old children (19\% of the population). The overall prevalence of obvious decay experience was 26, 25, 18 and $17 \%$, respectively, in the four years, with social inequalities apparent. Raw analyses revealed a drop in absolute inequality, but little change in relative inequality. The SII values over the four year period were $-1.61,-1.38,-1.16$ and -0.54 , respectively. Odds ratios from the LR models provide relative estimates (backed up by the ROC plots), the SIC and the PAR are good at picking up the absolute reduction, the Concentration Index does not add much information, the GINI coefficient does pick up inequality but is counter-intuitive in this context, and the SII and RII are useful. We found that standard analyses such as LR and ROC are good for estimating dental inequalities, but other methods are needed to more graphically demonstrate reductions in absolute inequality. Clyde.

\section{5 \\ Patterns of Anterior and Posterior Caries by Socioeconomic Status in Three-Year-Old Children}

A.D. McMahon ${ }^{\mathrm{a}, *}$, Y. Blair ${ }^{\mathrm{b}}$, D.R. McCall ${ }^{\mathrm{b}}$, L.M.D. Macpherson ${ }^{\mathrm{a}}$

alex.mcmahon@glasgow.ac.uk

aCommunity Oral Health, Glasgow Dental School, School of

Medicine, College of Medicine, Veterinary and Life Sciences,

University of Glasgow, and ${ }^{b}$ Oral Health Directorate, NHS

Greater Glasgow and Clyde, Scotland

The aim was to examine if anterior/posterior patterns of decayed missing and filled teeth in three-year-old children in Greater Glasgow and Clyde differ by socioeconomic status and by calendar time. The children were inspected in 2006/7,2007/8, 2008/9 and in 2009/10. A mean d3mft score was created for the four posterior teeth in the upper arch (varying from zero to one) and also for the two central incisors in the upper arch (also varying from zero to one). The difference between the anterior and posterior scores was calculated. Additionally, an endpoint of the occurrence of obvious caries experience in both the anterior and posterior teeth was created for the upper arch. The analyses were repeated within the quintiles of the Scottish Index of Multiple Deprivation. We sampled a total of 10,022 children. The anteriorposterior difference was statistically greater for the most deprived children versus the most affluent children; adjusted mean difference of differences $=0.03(0.02,0.05), \mathrm{p}<0.001$. These differences have reduced with calendar time, and by $2009 / 10$ there was no anterior-posterior difference (mean $=0.00$ for both the most deprived and the most affluent children). Over the four year period, there was more caries in both the anterior/posterior teeth for deprived children $(6 \%)$ than in affluent children (1\%); adjusted odds-ratio $=5.76(3.68,9.03), \mathrm{p}<0.001$. This effect was also reduced by $2009 / 10$. There is evidence that the pattern of decay in the upper arch is different in more deprived communities, with a higher level of caries in the anterior teeth and more decay in both the posterior and anterior teeth at the same time. These effects were reduced by calendar time as caries prevalence in the population reduced. Clyde.

The data collection was funded by NHS Greater Glasgow and

\section{6 \\ Inequalities in Dental Caries Distribution between Fluoridated and Non-Fluoridated Regions in Chile in 2008 \\ G. Rodríguez*, R. Cabello, I. Urzúa, S. Faleiros \\ grodrigu@odontologia.uchile.cl \\ Department of Restorative Dentistry, Faculty of Dentistry, \\ University of Chile, Santiago, Chile}

Aim: To compare the prevalence, severity and distribution of caries between 6-year-old children resident in fluoridated and non-fluoridated regions in Chile and to describe inequality measures between these regions. Methodology: Cross sectional oral health survey of a representative, random, stratified sample of 
6-year-old children of a fluoridated and a non-fluoridated region in Chile. Two calibrated dentist using artificial light and a plane dental mirror performed dental examination. World Health Organization (WHO) diagnostic criteria were used to assess tooth surfaces. Prevalence of caries free, $\mathrm{dmft}, \mathrm{SiC}$, proportion of $\geq 4 \mathrm{dmft}$ and of $\geq 7 \mathrm{dmft}$ were calculated and compared, and inequality measures like Gini coefficient and gap analysis of SES were calculated. Results: In the Bio Bio region (non-fluoridated) prevalence of caries free children was $41.11 \%$ (34.1-48.1) and in Valparaiso region (fluoridated) was $29.60 \%(23.9-35.2)$ with $\mathrm{p}=$ 0.11 . The mean of dmft in Bio Bio region was 3.85 (3.39-4.30) and in Valparaiso region was $2.79(2.34-3.24)$ with $p<0.01$. The mean of $\mathrm{SiC}$ index dmft in Bio Bio region was $8.5(7.68-8.62)$ and in Valparaiso region was $6.75(6.25-7.25)$ with $\mathrm{p}<0.01$. The high caries proportion $(4 \geq \mathrm{dmft})$ and rampant caries proportion (7 $\geq \mathrm{dmft})$ was $48.8 \%(42.5-55.1)$ and $24.4 \%(19.1-29.7)$ in nonfluoridated region respectively, and in fluoridated region was $36.0 \%(29.2-42.3)$ and $17.2 \%(11.9-22.5)$ with $\mathrm{p}<0.01$ for high caries proportion and $\mathrm{p}=0.06$ for rampant caries proportion. Gini coefficient for Valparaíso was 0.61 and for Bio Bio was 0.51 and gap analysis of SES for Valparaíso was $80.8 \%$ and for Bio Bio was $63.7 \%$. Conclusions: Caries levels measured in $\mathrm{dmft}$ index, $\mathrm{SiC}$ index and high caries proportion in 6-year-old children was significantly lower in a fluoridated region than a non-fluoridation region in Chile in 2008, but differences among SES were higher in fluoridated region than in non-fluoridated one, which suggest the presence of more inequalities in caries dental distribution at fluoridated regions.

\section{7}

\section{Caries Prevalence and Tooth Loss in a Chilean Adult} Population

\author{
I. Urzua a, *, C. Mendoza a , J. Gamonal ${ }^{\mathrm{a}}$, G. Rodríguez ${ }^{\mathrm{a}}$, R. Cabello , \\ S. Faleiros ${ }^{\mathrm{a}}$, P. Carvajal ${ }^{\mathrm{a}}$, A. Muñoz ${ }^{\mathrm{a}}$, I. Espinoza ${ }^{\mathrm{a}}$, W. Aranda ${ }^{\mathrm{b}}$, \\ O. Arteagab \\ ivanurzua@yahoo.com \\ ${ }^{\text {a } F a c u l t y ~ o f ~ D e n t i s t r y ~ a n d ~}{ }^{\mathrm{b} S} \mathrm{School}$ of Public Health, Faculty of \\ Medicine, University of Chile, Santiago, Chile
}

The purpose of this study was to assess the prevalence of dental caries, tooth loss and risk factors among Chilean adult population. Age, gender and behavioural specific differences in caries prevalence and tooth loss were also examined. A national stratified multistage probability design of the Chilean population in two age cohorts was applied. A sample of 1,553 adults, comprising 1,088 individuals aged 35-44 years and 465 individuals aged 6574 years were examined. The DMFT was evaluated following WHO recommendations using diagnostic criteria at the caries into dentin threshold. The data were analyses by univariate and multivariate models using logistic regression analyses. Results showed a mean DMFT 15.06 in the 35-44 years-old group and increasing to 21.57 at $65-74$ years. Factors related with tooth loss in 35-44 years-old in univariate logistic regression were depression (OR 1.9; CI 95\%: 1.26-2.85), education level $<12$ years (OR 2.24; 95\% CI: 1.31-3.73), personal income (OR 1.51; 95\% CI: $1.04-$
2.19) and family income (OR 2.05; 95\% CI: 1.34-3.13) and for multivariate logistic regression in the same age group were depression (OR 1.93; 95\% CI: 1.24-3.0), education level $<12$ years (OR 1.94; $95 \%$ CI: $1.2-3.14$ ) and family income (OR 1.71; 95\% CI: 1.09-2.68). Factors related with tooth loss in 65-74-year-olds in univariate logistic regression were education level $<12$ years (OR 2.54; $95 \%$ CI: 1.3-4.96) and personal income (OR 1.66; 95\% CI: 1.05-2.63) and for multivariate logistic regression in the same age group were education level $<12$ years (OR 2.51; 95\% CI: $1.21-5.18$ ). In conclusion, the adult population in Chile showed a high prevalence of dental caries with age, education level, personal and family income and depression the main risk indicators of tooth loss.

\section{8 \\ The Prevalence of Tooth Wear: A Prospective Cohort Study (Timepoints: 2000, 2007, and 2010)}

M. Harding ${ }^{\mathrm{a}, *}$, M. Cronin ${ }^{\mathrm{b}}$

m.harding@ucc.ie

aOral Health Services Research Centre, University Dental School and Hospital, and bepartment of Mathematical Sciences,

Aras na Laoi, University College Cork, Cork, Ireland

Aim: To establish the prevalence of tooth wear in a cohort of school-children examined in the years 2000, 2007 and 2010. Design: A prospective cohort study at three timepoints [tp]: 2000 [tp1], 2007 [tp2] and 2010 [tp3]. Experimental Approach: Tooth wear was measured using two scoring systems modified from the Smith and Knight Tooth Wear Index (TWI). This abstract reports tooth wear that had progressed to dentine, assessed on the occlusal surfaces of the four first permanent molars, the labial, lingual/ palatal and incisal surfaces of the six upper and lower anterior teeth. Demographic data were collected from the parents and an electronic questionnaire on oral hygiene, diet and behaviours completed by each child. The second index will be reported later (all teeth and both enamel and dentine were assessed with the second index). Results: At tp3 complete data were available for 85 children, mean age $14.7 \pm 0.3$ years, and could be compared with examinations at [tp1] and [tp2]. At [tp3], 48\% ( $\mathrm{n}=36)$ of subjects had toothwear; at [tp2] (mean age $12.0 \pm 0.3$ years), 31\% $(\mathrm{n}=19)$ had tooth wear. At [tp1] (mean age 5.47 \pm 0.3$), 20 \%(n=15)$ of the children had toothwear into dentine on the upper primary incisors. The prevalence when incisor teeth only were scored was: $44 \%$ $(\mathrm{n}=33)$ [tp3] and 27\% $(\mathrm{n}=17)$ [tp2]. When the occlusal surfaces of molar teeth only were scored $11 \%(n=8)$ had signs of tooth wear at [tp3] and $5 \%(n=3)$ at [tp2]. Conclusions: The prevalence of toothwear scored at the dentine level at [tp3] had increased but not the severity of tooth wear scored. Future analyses will compare the severity as assessed with the two indices, and associations with personal data collected.

Research facilitated by a GlaxoSmithKline Consumer Healthcare Research Grant. 


\section{9}

Oral Health Status and Treatment Needs among Hospitalized Psychiatric Patients

V. Bertaud ${ }^{\mathrm{a}, \mathrm{b}}$, *, C. Lesquibe-Perrus ${ }^{\mathrm{c}}$, F. Richard ${ }^{\mathrm{a}, \mathrm{b}}$

valerie.bertaud@univ-rennes1.fr

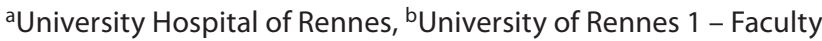
of Dentistry, and ' Guillaume Régnier Psychiatric Center, Rennes, France

This study aims at assessing oral health and treatment needs of patients attending a psychiatric ward. The target population was all the adult patients hospitalized in the general psychiatric wards of Rennes (741 beds, 659 patients in February 2006) which serves all the department of Ille-et-Vilaine (about 925,000 inhabitants in 2006). A list of all the beds was drawn up. A random sample of approximately $25 \%$ of the beds was chosen for examination (185 beds). By the day of examination, if there was no hospitalized patient corresponding to a sampled bed, the bed immediately after on the list was chosen instead. Caries prevalence (WHO criteria; DMF-T: number of decayed, missing or filled teeth), oral hygiene (OHIS: Simplified Oral Hygiene Index) and treatment needs were recorded. The unstimulated saliva flow rate was also assessed. Data analysis was undertaken using EPIInfo v3.3.21 and SPSS V17.0. The study population consisted of 161 patients who agreed to participate $(24.44 \%$ the adult psychiatric population in the department). They were from 16 to 90 years old (mean $46.9 \pm 17.5$ years). Among the 161 examined patients, mean DMF-T was $15.8 \pm 8.8(\mathrm{D}=3.7 \pm 4.4, \mathrm{M}=7.3 \pm 9.4, \mathrm{~F}=$ $4.7 \pm 4.9)$ and 18 patients $(11.2 \%)$ were edentulous. Among the 143 dentate patients, 122 (93.0\%) needed dental care: $41(28.7 \%)$ needed extractions (3.12 \pm 3.00 extractions per people), $114 \mathrm{pa}$ tients (79.7\%) needed conservative dental care (3.96 \pm 3.22 dental cares per person) and 31 patients needed crowns $(1.71 \pm 1.10$ crown per patient). The mean OHIS was $1.66 \pm 1.12$. Among the 147 patients who agreed to carry out the salivary examination, the average saliva flow rate was $1.57 \mathrm{~g}( \pm 1.42)$ in $5 \mathrm{~min}$. Hyposalivation $(<0.5 \mathrm{~g} / 5 \mathrm{~min})$ or low saliva flow rate $(<1 \mathrm{~g} / 5 \mathrm{~min})$ was found in 74 patients (50.3\%). The DMF-T is slightly higher than in French population (DMF-T $=14.6 ; \mathrm{D}=1.2 ; \mathrm{M}=3.0 ; \mathrm{F}=10.4$ ) but psychiatric patients have 3 times more decayed teeth, twice the number of missing teeth and half the number of filled teeth. Preventive actions could be targeted on people who are planned to stay a long time at the hospital and especially on plaque limitation (oral hygiene or antiseptic agents) or plaque action limitation (fluorides). Secondary prevention actions are also needed: frequent and systematized dental visit for screening and early caries treatment. Oral health care is especially difficult for those patients. Specific prevention protocols are strongly needed.

This study was partly funded by the CHU of Rennes and the Faculty of Dentistry of Rennes.

\section{0 \\ ICDAS II and Salivary Cariogenic Microflora in Caries Development in Riga's Children with Baseline Age 6 and 12 Years: A Follow-Up Study}

J. Gudkina a, b, *, S.H. Abrams d, e, A. Brinkmane ${ }^{\mathrm{a}, \mathrm{b}}$, I. Rogovska ${ }^{\mathrm{a}, \mathrm{b}}$, G. Locane $e^{c}$

j.gudkina@inbox.Iv

aRiga Stradins University, 'Institute of Stomatology, Riga, and 'Private dental office, Liepaja, Latvia; ${ }^{\mathrm{Q}}$ Quantum Dental

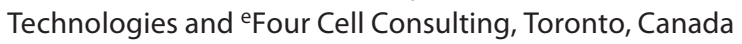

The aim of this study was to assess linkage between caries development and salivary cariogenic microflora in children initially aged $6(n=20)$ or $12(n=13)$ years using ICDAS II, and to evaluate the role of these variables in caries risk assessment. All children were examined at baseline and after 3 years. Total $\mathrm{dmft} /$ DMFT and dmfs/DMFS were recorded using bitewing radiographs. ICDAS II and salivary mutans streptococci (MS) and lactobacilli (LB) (CRT - bacteria, Ivoclar, Vivadent, Liechtenstein) were measured on all participants. The data were analyzed using chi-square tests and ANOVA. ANOVA evaluated how changes in variables such as salivary cariogenic microflora affect caries in particular age group. The mean (SD) dmfs/DMFS of approximal surfaces was 2.85 (2.52) in the original 6 year-olds and 3.28 (3.68) in the original 12 year-olds; occlusal surfaces it was 1.15 (1.95) in original 6 year-olds and 1.28 (1.38) in original 12 year-olds and for the buccal/lingual surfaces it was 0.45 (0.83) and 0.50 (0.94), respectively accordingly. According to ICDAS II the mean number (SD) of carious approximal surfaces was $21.8(6.32)$ in original 6 year-olds and 30.57 (7.87) in original 12 year-olds; occlusal surfaces 2.6 (2.44) in original 6 year-olds and 7.43 (3.94) in original 12 year-olds and buccal/lingual surfaces 18.55 (5.34) and 25.21 (9.60), respectively. Significant difference between age groups was found for each surface $(p=0.002)$. Significant associations were observed between cavitated lesions (ICDAS II) and salivary LB in 6 year-olds ( $p=0.007)$; all cavitated lesions (ICDAS II) in both age groups and salivary LB $(\mathrm{p}=0.032)$. There is an association between caries development and salivary cariogenic microflora, and ICDAS is an effective tool assigning caries development, which should be considered in caries risk-based preventive programs.

\section{1 \\ Current Concepts in Cariology Teaching in Latin America \\ S. Martignon ${ }^{\mathrm{a}, *}$, J.A. Ruiz ${ }^{\mathrm{a}}$, M.C. Rangel ${ }^{\mathrm{a}}$, M. Tellez ${ }^{\mathrm{b}}$, J. Gomez ${ }^{\mathrm{a}}$, S. Falla ${ }^{\mathrm{a}}$, L. Marin ${ }^{\mathrm{a}}$ \\ smartignon@gmail.com \\ ${ }^{a}$ Caries Research Unit UNICA, Universidad El Bosque, Bogotá, Colombia; ${ }^{\text {b}}$ Maurice H. Kornberg School of Dentistry, Temple University, Philadelphia, Pa., USA}

Objective: To assess current cariology teaching practice amongst Latin American dental schools. Materials and Methods: A 20 -item questionnaire on several teaching aspects (cur- 
riculum, diagnosis, treatment, and perception) was mailed to dental schools affiliated to OFEDO (Dental Schools, Faculties and Departments Organization) ( $\mathrm{n}=54)$, and non-affiliated schools $(\mathrm{n}=30)$. The questionnaire was developed with the help of 5 experts (comprehension, content, language), and two focus groups were held with regional researchers/teachers. Results: The response rate was $60 \%,(n=50$; OFEDO: $n=20$; others: $n=$ 30) after 3 mailings. The distribution by country was as follows: Bolivia (4); Chile (4); Colombia (21); Costa Rica (1); Cuba (1); El Salvador (2); México (5); Panamá (2); Perú (4); Puerto Rico (1); República Dominicana (2); Uruguay (2); Venezuela (1). About $44 \%$ of the schools considered cariology the key axis of the course, with this being lead less frequently by Cariology Departments (22\%) compared to other departments (Paediatric, Community, Microbiology, Operative-Dentistry); all schools use a cariology textbook/guideline; $80 \%$ have preclinical practices, but only $32 \%$ claimed to transfer to the clinical practice the theoretical concepts. The most frequently reported diagnostic methods taught were visual-tactile (94\%), with $60 \%$ of school incorporating ICDAS criteria in this teaching. Forty percent incorporated radiographic caries diagnosis. All schools reported teaching caries risk assessment for diagnostic purposes. A surgical intervention was advocated in $44 \%$ of schools when there is visually any structure disruption to the surface, and $44 \%$ when there is radiolucency at the enamel-dentine junction. All schools reported teaching prevention strategies, but only in $40 \%$ of schools was this linked to risk assessment; $44 \%$ implement nonsurgical management occasionally. Finally, $62 \%$ of the sample felt that cariology is not properly addressed in their school curriculum. Conclusion: This questionnaire will help improving the Latin American dental-school curriculum by identifying failures (low theory-to-clinic transfer), gaps (low preventionrisk-assessment link), and opportunities (low cariology-addressing perception in the curriculum).

Universidad El Bosque partially funded this project. 


\section{Caries Research}

\section{Session 5 Erosion}

\section{2 \\ Casein as an Agent to Inhibit Hydroxyapatite Dissolution}

\author{
A.J. White ${ }^{\mathrm{a},}$, L.H. Gracia ${ }^{\mathrm{b}}$, M.E. Barbour ${ }^{\mathrm{a}}$ \\ a.white@bristol.ac.uk \\ aDepartment of Oral and Dental Science, University of Bristol, \\ and ${ }^{b}$ GlaxoSmithKline R\&D, Weybridge, UK
}

The aim of this study was to determine whether treating hydroxyapatite (HA) discs with commercially available casein and casein-derived peptides, with and without fluoride, could inhibit HA dissolution in citric acid. An automatic titration system known as a $\mathrm{pH}$ stat was used to maintain a constant $\mathrm{pH}$ and track the dissolution rate of $\mathrm{HA}$ discs. A reaction temperature of $36^{\circ} \mathrm{C}$ was maintained. Four HA discs were used per treatment group. Baseline dissolution rates for $30 \mathrm{~min}$ in $0.30 \% \mathrm{w} / \mathrm{v}$ citric acid $\mathrm{pH}$ 3.20 were initially examined, then the discs were exposed to solutions of: $0.50 \% \mathrm{w} / \mathrm{v}$ casein, casein phosphopeptide (CPP) or glycomacropeptide (GMP) (with and without $300 \mathrm{ppm} \mathrm{F}$ as NaF) and $300 \mathrm{ppm} F$ for $10 \mathrm{~min}$, or $0.50 \% \mathrm{w} / \mathrm{v}$ casein for $30 \mathrm{~s}$ and $30 \mathrm{~min}$. Discs were then exposed to the citric acid for a further $30 \mathrm{~min}$ and percentage difference in dissolution rate, before and after treatment, obtained. Mineral loss was linear with respect to time over the exposure period. All treatments resulted in a statistically significant $(\mathrm{p}<0.05)$ reduction in dissolution rate as determined using ANOVA and Newman-Keuls. A 10 min treatment of $0.5 \%$ $\mathrm{w} / \mathrm{v}$ casein resulted in a $43.9 \%( \pm 13.4)$ reduction in dissolution rate. With the addition of $300 \mathrm{ppm} F$ this increased to a statistically significantly superior $81.7 \%( \pm 20.8)$ reduction. Treatment time had no statistically significant impact. In conclusion, $0.5 \%$ $\mathrm{w} / \mathrm{v}$ casein reduced the dissolution rate of HA discs in citric acid and this effect is additive in the presence of fluoride. Exposure time was found not to have a significant effect, having useful implications for casein's use as a preventative oral treatment.

Financial support received from the EPSRC and GSK Consumer Healthcare.

\section{3 \\ Analysis of the Erosive Effect of Different Dietary Substances and Medicaments}

A. Lussi*, B. Megert, R.P. Shellis, X. Wang

adrian.lussi@zmk.unibe.ch

Department of Preventive, Restorative and Pediatric Dentistry, University of Bern, Switzerland

Excessive consumption of acidic foods and drinks contributes to tooth erosion. The aims of this in vitro study were two-fold: (1) to assess the erosive potential of different dietary substances and medicaments; (2) to determine chemical properties with an impact on the erosive potential. Sixty substances were selected: soft drinks, energy drinks, sports drinks, alcoholic drinks, juices, fruits, mineral waters, yoghurts, teas, coffee, salad dressings and medicaments. Surface nanohardness (SNH) of enamel specimens was measured before and after immersion for 2 and $4 \mathrm{~min}$ in the tested products. The erosive potential of a given substance was quantified as the changes in $\mathrm{SNH}$ after 2 or 4 min exposure $\left(\Delta \mathrm{SNH}=\mathrm{SNH}_{2 \mathrm{~min} / 4 \mathrm{~min}}-\mathrm{SNH}_{\text {baseline }}\right)$. To characterize these substances, various chemical properties, e.g., $\mathrm{pH}$, concentrations of calcium $(\mathrm{Ca})$, total phosphate $\left(\mathrm{P}_{\mathrm{i}}\right)$ and fluoride $(\mathrm{F})$, titratable acidity to $\mathrm{pH} 7.0$ and buffering capacity at the original $\mathrm{pH}$ value $(\beta)$, as well as degree of saturation (pK-pI) with respect to hydroxyapatite and fluorapatite, were determined. Erosive challenge caused a statistically significant reduction in SNH for all substances except for coffee, some medicaments and alcoholic drinks, and non-flavoured mineral waters, teas, and yoghurts. By multivariate linear regression analysis, $52 \%$ of the variation of the changes in SNH after 2 min and 61\% after 4 min immersion were explained by $\mathrm{pH}, \beta$, and concentrations of $\mathrm{Ca}$ and $\mathrm{F}(\mathrm{p}<0.05)$. The $\mathrm{pH}$ was the variable with the highest impact in the multiple regression and in the bivariate correlation analyses. Furthermore, a high bivariate correlation was also obtained between $(\mathrm{pK}-\mathrm{pI})_{\mathrm{HAP}}$, $(\mathrm{pK}-\mathrm{pI})_{\mathrm{FAP}}$ and $\Delta \mathrm{SNH}$.

This study was supported by the Swiss Dental Society of Odontology (SSO) (Project Nr. 222-05). 


\section{4}

The Erosion- and Abrasion-Inhibiting Effect of Dentifrices with Different Fluoride Concentrations and $\mathbf{p H}$ in vitro

A.C. Magalhães*, B.M. Moron, S. Satie, N. Ito, F. Vilhena, M.A.R. Buzalaf

acm@usp.br

Department of Biological Sciences, Bauru School of Dentistry, University of São Paulo, Bauru, Brazil

This in vitro study analysed the effect of increasing concentrations of fluoride in acidic or neutral liquid dentifrices to protect against enamel and dentine erosion and abrasion. One hundred and thirty-two bovine enamel and root dentine specimens were randomly allocated to the following toothpastes: experimental liquid dentifrices with $550 \mu \mathrm{g} \mathrm{F} / \mathrm{g}(\mathrm{pH} 4.5) ; 550 \mu \mathrm{g} \mathrm{F} / \mathrm{g}(\mathrm{pH} \mathrm{7.0)}$; $1,100 \mu \mathrm{g} \mathrm{F} / \mathrm{g}$ (pH 4.5); 1,100 $\mu \mathrm{g} \mathrm{F/g} \mathrm{(pH} \mathrm{7.0);} \mathrm{5,000} \mathrm{\mu g} \mathrm{F/g} \mathrm{(pH} \mathrm{4.5);}$ $5,000 \mu \mathrm{g} \mathrm{F} / \mathrm{g}$ ( $\mathrm{pH} 7.0$ ); placebo ( $\mathrm{pH} 4.5$ ); placebo (pH 7.0); and commercial toothpastes with $550 \mu \mathrm{g} \mathrm{F/g}$ (Colgate Baby Barney, $\mathrm{pH}$ 7.0); 1,100 $\mu \mathrm{g} \mathrm{F/g}$ (Crest, pH 7.0); and 5,000 $\mu \mathrm{g} \mathrm{F/g} \mathrm{(Duraphat,}$ $\mathrm{pH}$ 7.0). Over 7 days, the specimens were subjected to an erosive demineralisation (Zero Sprite, $\mathrm{pH}$ 2.6) for $90 \mathrm{~s}, 4$ times a day. Additionally, the specimens were abraded by brushing for $15 \mathrm{~s}$ using one of the dentifrices (liquid dentifrices: $\sim 0.15 \mathrm{~g} / \mathrm{commercial}$ toothpastes: $\sim 0.4 \mathrm{~g}), 2$ times a day. Dental loss was measured using a contact profilometry $(\mu \mathrm{m})$. Data were analysed by ANOVA/ Tukey's test and two-way ANOVA/Bonferroni's test ( $\mathrm{p}<0.05)$. All fluoridated liquid dentifrices significantly reduced enamel loss (5.5-6.4 $\mu \mathrm{m})$ compared to the placebo and commercial dentifrices $(8.8-9.8 \mu \mathrm{m})(\mathrm{p}<0.0001)$. Only liquid dentifrices with 1,000 $\mu \mathrm{g} \mathrm{F} / \mathrm{g}$ and 5,000 $\mu \mathrm{g} \mathrm{F/g} \mathrm{(both} \mathrm{pH}$ ) significantly reduced dentine loss $(4.6-4.8 \mu \mathrm{m})$ compared to the placebo dentifrice (5.3$5.6 \mu \mathrm{m})(\mathrm{p}<0.0001)$. The $\mathrm{pH}$ had no effect, but the consistency had considerable impact on the effect of dentifrices $(p<0.05)$. Thus, it can be concluded that liquid dentifrices, especially with high $\mathrm{F}$ concentration, seem to be a good option for reducing dental loss by mild erosive and abrasive challenges in vitro.

FAPESP (Process numbers 2009/07470-7 and 2009/08400-2).

\section{5 \\ The Erosive Effects of Acid Beverages and Oral Antibacterial Solutions on Dental Hard Tissues \\ S. Andrian ${ }^{\mathrm{a}, *}$, S. Stoleriu a, G. lovan ${ }^{\mathrm{a}}$, A. Georgescu ${ }^{\mathrm{a}}$, A.V. Sandu ${ }^{\mathrm{b}}$ \\ sorinandrian@yahoo.com \\ aDepartment of Odontology and Periodontology, Dental Medicine School, Gr.-T.-Popa-University of Medicine and Pharmacy, and baterials Science and Engineering Faculty, Gh.-Asachi-Technical University, Iaşi, Romania}

The aims of the study were: (1) to evaluate the $\mathrm{pH}$ and titrable acidity of three acidic beverages (Nestea lemon, Coca-Cola and red wine) and of two oral antibacterial mouthwashes (Listerine and Corsodyl); (2) to determinate the calcium and phosphate ion concentration in enamel and dentine before and after exposure; (3) to evaluate the erosive effect of solutions on dental hard tissues.
$\mathrm{pH}$ and titrable acidity of solutions were established using an electronic $\mathrm{pH}$ metre (HANNA pH 210). 36 molars extracted from periodontal or orthodontic reasons were used in this study. The control group included 12 molar halves placed in distilled water. Each study group included 12 molar halves placed in three acidic beverages for $30 \mathrm{~min}$ and in two oral antibacterial solutions for $14 \mathrm{~min}$. The calcium and phosphate ion concentrations in enamel and dentine were determined using EDX detector QUANTAX QX2 (Bruker/Roentec, Germany). The teeth sections were analyzed using SEM (VEGA II LSH, Tescan, Czech Republic) to evaluate the morphological changes after acid solutions action. CocaCola had the highest level of titrable acidity $(0.580 \mathrm{ml})$ and the lowest $\mathrm{pH}$ value $(2.85 \mathrm{ml})$, followed by red wine $(0.330 \mathrm{ml}) \mathrm{pH} 3.9$, Nestea lemon $(0.300 \mathrm{ml}) \mathrm{pH} 3.8$, Listerine $(0.110 \mathrm{ml}) \mathrm{pH} 4.35$ and Corsodyl $(0.040 \mathrm{ml}) \mathrm{pH}$ 5.6. Significant decreases of calcium and phosphate ion concentrations were obtained for samples placed in test solutions compared to control group (paired samples t test). SEM images clearly showed the erosive effect of Coca-Cola, red wine, Nestea and Listerine, with enamel surface irregularities and marked opening of the dentine tubules. Corsodyl caused only minor surface changes. This study indicates that these common acidic beverages and antibacterial solutions may soften and erode dental hard tissues.

This study was supported by Dental Medicine School, University of Medicine and Pharmacy, Iaşi. The authors thank Oral and Maxillofacial Surgery Department of Dental Medicine School for their effort to collect the teeth used in this study.

\section{6 \\ Salivary Parameters after Vomiting in Persons with Bulimia Nervosa: A Controlled Clinical Trial}

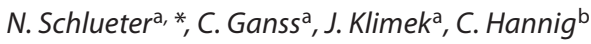

nadine.schlueter@dentist.med.uni-giessen.de

aDepartment of Conservative and Preventive Dentistry, Dental

Clinic, Justus-Liebig-University of Giessen, and ${ }^{b} \mathrm{Clinic}$ of

Conservative Dentistry, Faculty of Medicine 'Carl Gustav Carus', Technical University of Dresden, Germany

Little is known about salivary parameters in patients with bulimia, particularly directly after vomiting. Aim of the study was to compare salivary flow-rate, $\mathrm{pH}$ and buffering-capacity of persons with bulimia in comparison to healthy controls. Twentyeight subjects participated (14 with bulimia, seven with severe and seven without erosion; 14 matched healthy individuals). From all participants, resting and stimulated saliva was analysed; additionally, from the bulimic patients, saliva collected directly and $30 \mathrm{~min}$ after vomiting, was investigated. Flow-rate of resting saliva was significantly lower in patients with bulimia $(\mathrm{p}<0.05)$. However, there was no difference in stimulated saliva. For $\mathrm{pH}$, no significant difference was found for resting saliva, but $\mathrm{pH}$ of stimulated saliva was significantly higher in bulimic persons than in controls $(p<0.01)$. For buffering-capacity, no significant difference was found between groups as well for resting as for stimulated saliva. In bulimic patients, the flow-rate directly after vomiting was $125 \%$ (after $30 \mathrm{~min}$ : 72\%) higher than that of resting saliva, but $59 \%$ (69\%) lower than that of stimulated saliva (all dif- 
ferences $\mathrm{p}<0.01)$. In the bulimic group, values for $\mathrm{pH}$ and buffering-capacity of saliva directly after vomiting were similar to these of stimulated saliva (n.s.); values of saliva collected $30 \mathrm{~min}$ after vomiting were similar to these of resting saliva (n.s.). A subgroup analysis of the bulimic patients revealed, that subjects with erosion had a significantly lower buffering-capacity $(p<0.05)$ directly after vomiting. In conclusion, only small changes in parameters were found between healthy and bulimic participants. The lower buffering-capacity of the saliva directly after vomiting in patients suffering from bulimia nervosa and erosion indicate that this parameter could be a predisposing factor for the formation of erosion in bulimic patients.

Supported by Deutsche Gesellschaft für Zahn-, Mund- und Kieferheilkunde (DGZMK).

\section{7 \\ Profilometry in Eroded Human Dentine: Impact of Measuring Conditions}

K. Schulze*, N. Schlueter, J. Klimek, C. Ganss

katja.schulze@dentist.med.uni-giessen.de

Department of Conservative and Preventive Dentistry, Dental

Clinic, Justus-Liebig-University, Giessen, Germany

Profilometry is established in erosion research. When used in dentine, however, factors such as demineralised surface material (DSM) and drying effects may interfere with profilometric procedures. The study sought to evaluate the impact of sample preparation and handling, and type of stylus on measurement results. Dentine specimens (standardised thickness) were eroded with citric acid (0.05 M, pH 2.6) for 5, 10, 15, or 20 minutes ( $\mathrm{n}=15$ each). On each sample, tissue loss was determined under various conditions: before and after the enzymatic removal of DSM, under standardised wet and ambient air conditions, and with non-contact and contact profilometry. In the presence of DSM and keeping samples moist, the non-contact stylus revealed almost no tissue loss. Values ( $\mu \mathrm{m}$, mean $\pm \mathrm{SD})$ after $5,10,15,20$ min were $0.3 \pm$ $0.7,0.4 \pm 1.2,0.5 \pm 1.3,0.8 \pm 1.7$ resp. The contact stylus increased values significantly to $2.9 \pm 1.1,4.9 \pm 1.3,6.9 \pm 1.6,9.0$ \pm 1.7 resp. Exposure to ambient air $(2 \mathrm{~min})$ significantly increased values for non-contact $(0.8 \pm 1.3,2.7 \pm 1.6,4.5 \pm 1.7,7.0$ \pm 1.8 resp.), and decreased values for contact profilometry $(0.9$ $\pm 1.2,3.4 \pm 1.3,5.2 \pm 2.2,8.5 \pm 2.2$ ). After removal of DSM (moist conditions), values were distinctly higher (contact stylus: $4.2 \pm 1.3,7.1 \pm 1.6,11.1 \pm 2.0,14.8 \pm 2.5$ resp.; non-contact stylus: $3.5 \pm 0.8,6.1 \pm 1.2,9.6 \pm 1.8,13.2 \pm 2.6)$. Drying in ambient air lowered values by about $2-4 \mu \mathrm{m}$ due to warping. BlandAltmann-comparisons of the various outcomes revealed distinct significant proportional and relative biases. Loss of mineralised tissue is not adequately quantified unless DSM is removed and samples are kept moist. Most importantly, values obtained in presence or absence of DSM were not proportional. Exposure to ambient air rapidly resulted in changes of measuring outcomes. The study emphasises the need for adequate sample preparation and choice of stylus depending on the parameter under study, and for meticulous standardisation.

Justus-Liebig University, Giessen, Germany.

\section{8}

\section{Effect of Different Mouthrinses on Repairing Enamel Defects Caused by Acidic Attacks}

\author{
G. Nagy ${ }^{\mathrm{a}, *}$, A. Zsigmond ${ }^{\mathrm{a}}$, B. Palasti Kovacs ${ }^{\mathrm{c}}$, C. Hegedüs $^{\mathrm{d}}$ \\ S. Márton ${ }^{\mathrm{e}}$, M. Madléna ${ }^{\mathrm{b}}$
}

nagyga@net.sote.hu

aSection of Oral Diagnostics and bepartment of Pediatric Dentistry and Orthodontics, Faculty of Dentistry, Semmelweis University, Budapest, Institute of Mechanical Engineering and Safety Techniques, Donát Bánki Faculty of Mechanical Engineering and Security Technology, Óbuda University, Budapest, ${ }^{d}$ Department of Prosthetic Dentistry, Faculty of Dentistry, University of Debrecen Medical School and Health Science Center, Debrecen, and e Department of Sociology and Social Policy, Faculty of Arts, University of Debrecen, Debrecen, Hungary

For prevention of erosion several novel agents has been developed, but their efficacy still is not clarified exactly, so we aimed it in our experiment. The objective of the present study was the evaluation of three mouthrinses (Elmex erosion protection, BioRepair, Pronamel) on erosion process of enamel. We prepared enamel samples from impacted third molars and these were exposed to a cyclic de-and remineralization procedure for five days $(0.05 \mathrm{M}$ citric acid, $\mathrm{pH}$ 2.3). Except the control group, the specimen were treated with different test solutions after the first and the sixth demineralizing cycle. Each examination group consisted of ten specimen. Enamel defects were determined after the last experimental day profilometrically (Perthometer S8P, with a mechanical stylus). The control group showed the greatest tissue loss 29.2 $\pm 6.1 \mu \mathrm{m}$ (mean $\pm \mathrm{SD}$ ). All of the tested solutions proved to be effective in preventing the enamel loss $(\mathrm{p}<0.01)$, although the differences between the test mouthrinses were not statistically significant $(13.7 \pm 3.2 \mu \mathrm{m}, \mathrm{p}<0.0001$ in case of Elmex erosion protection, $18 \pm 6.9 \mu \mathrm{m}$ in case of BioRepair and $18.4 \pm 6.1 \mu \mathrm{m}$ in case of Pronamel p $<0.002$, versus the control group). All of the investigated mouthrinses could reduce of erosion of dental enamel. Rate of reduction of lesion depth was nearly $15 \%$ higher using Elmex antierosive, than in case of Biorepair or Pronamel mouthrinses. The difference was statistically significant between the Elmex antierosive and Pronamel groups $(\mathrm{p}<0.05)$.

The authors greatly acknowledge the support of GABA International Ltd, Therwil, Switzerland. 


\section{9}

\section{Influence of Brushing on the Erosion-Protective Effect of Different Fluoride- or Chloride-Containing Compounds}

\author{
A. Wiegand ${ }^{\mathrm{a}}$ * , S. Schneider ${ }^{\mathrm{a}}$, B. Sener ${ }^{\mathrm{a}}$, M. Roos $^{\mathrm{b}}$, T. Attin $^{\mathrm{a}}$ \\ annette.wiegand@zzm.uzh.ch \\ ${ }^{a}$ Clinic for Preventive Dentistry, Periodontology and Cariology \\ and ${ }^{b}$ Division of Biostatistics, Institute of Social and Preventive \\ Medicine, University of Zurich, Switzerland
}

This in vitro study aimed to analyse the impact of brushing on the protective effect of different fluoride- or chloride-containing compounds on enamel and dentin erosion. Bovine enamel and dentin samples were treated once with $\mathrm{TiF}_{4}, \mathrm{AmF}, \mathrm{SnF}_{2}(0.5 \mathrm{M} \mathrm{F}), \mathrm{SnCl}_{2}$ or $\mathrm{SrCl}_{2}(0.5 \mathrm{M} \mathrm{Cl})$ solutions for $2 \mathrm{~min}$ or with $\mathrm{AmF}$ followed by $\mathrm{SnCl}_{2}$ or $\mathrm{SrCl} 2$, respectively (each $60 \mathrm{~s}$ ). The solutions were applied at their native $\mathrm{pH}: \mathrm{TiF}_{4}: 1.3, \mathrm{AmF}: 4.3, \mathrm{SnF}_{2}: 2.6, \mathrm{SnCl}_{2}: 1.5, \mathrm{SrCl}_{2}$ : 4.9. Samples rinsed with water served as control. Then, samples were either left unbrushed or were brushed with $10,20,50,100$ or 500 brushing strokes in an automatic brushing machine at $2 \mathrm{~N}$ using a non-fluoridated toothpaste slurry (RDA: 50, REA: 6, each group $\mathrm{n}=10)$. All samples were eroded with $\mathrm{HCl}(\mathrm{pH} 2.3)$ for $60 \mathrm{~s}$. Calcium release into the acid was determined by atomic absorption spectroscopy and statistically analysed by ANOVA and Bonferroni post-hoc tests $(p<0.05)$. Calcium release of unbrushed samples was significantly reduced by $\mathrm{AmF}, \mathrm{TiF}_{4}, \mathrm{SnF}_{2}, \mathrm{SnCl}_{2}$ and $\mathrm{AmF}+\mathrm{SnCl}_{2}$ (58-67\% of control) in enamel and by all test solutions $(23-86 \%$ of control) in dentin. In most groups, calcium release increased with increasing brushing strokes prior to acid application. After 500 brushing strokes, enamel erosion was reduced by $\mathrm{AmF}, \mathrm{TiF}_{4}, \mathrm{SnCl}_{2}$ and $\mathrm{AmF}+\mathrm{SnCl}_{2}$ to only $70-85 \%$ of control, while calcium loss in dentin was decreased by AmF, TiF $4, \mathrm{SnCl}_{2}, \mathrm{SnF}_{2}$ and $\mathrm{AmF}+\mathrm{SnCl}_{2}$ to $45-78 \%$ of control. $\mathrm{SrCl}_{2}$-containing solutions and $\mathrm{SnF}_{2}$ (in enamel) were ineffective to reduce calcium loss after application of 500 brushing strokes. The protective potential of fluoride- and chloride-containing solutions to prevent enamel and dentin erosion varies significantly and is affected by brushing to different extent.

\section{0 \\ The Effect of Topical Fluorides on Enamel Volume Loss from Erosion and Erosion-Abrasion in vitro}

K.R. Stenhagen ${ }^{\mathrm{a},}{ }^{*}$, R.S. Austin ${ }^{\mathrm{b}}$, L.H. Hove ${ }^{\mathrm{a}}$, A.B. Tveit ${ }^{\mathrm{a}}$, F. Festy ${ }^{\mathrm{b}}$, R. Moazzez ${ }^{\mathrm{b}}$, D.W. Bartlett ${ }^{\mathrm{b}}$

k.r.stenhagen@odont.uio.no

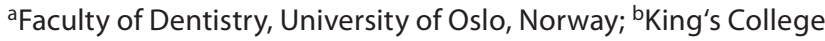
London Dental Institute, UK

Reduced erosive enamel wear has been demonstrated in vitro and in situ following topical applications of fluorides. Little is known about the protective effect during intermittent erosion and abrasion. The aim was to investigate the protective effect of highly concentrated fluoride solutions and a varnish on enamel in a cyclic erosion and erosion-abrasion model. 60 polished human enamel specimens (12 per group) were covered with polyvinyl tape to create a central $1 / 3$ area for treatment with $\mathrm{NaF}(\mathrm{pH}$
8.0), $\mathrm{SnF}_{2}$ (pH 2.6) or $\mathrm{TiF}_{4}$ ( $\mathrm{pH}$ 1.2) $0.5 \mathrm{M} \mathrm{F}$ solutions for $2 \mathrm{~min}$, Bifluorid $10{ }^{\circledR}$ varnish $\left(5 \% \mathrm{NaF}+5 \% \mathrm{CaF}_{2}, \mathrm{pH} 5.5\right)$ for $20 \mathrm{~s}$ or deionised water (negative control). 2/3 remained as bilateral reference areas. 30 specimens $(6 \times 5)$ were allocated to an erosion protocol (one cycle was $2 \mathrm{~min}$ in $0.01 \mathrm{M} \mathrm{HCl}$ and $60 \mathrm{~min}$ remineralisation in artificial saliva and 30 specimens $(6 \times 5)$ to an erosion-abrasion protocol (similar to the erosion cycle, followed by tooth brushing with fluoride free toothpaste slurry $(200 \mathrm{~g}, 120$ linear strokes) for $2 \mathrm{~min}$ ). The tape was removed and a $5 \times 3 \mathrm{~mm}$ central area was scanned by a white light con-focal profilometer with a $7 \mu \mathrm{m}$ spot size after the $3 \mathrm{rd}$, 6th and 9th cycles. The distance between individual data points was $10 \mu \mathrm{m}$. 3D data was analysed with volume loss as outcome. The tape was replaced between the respective cycles. The $\mathrm{TiF}_{4}$ solution and Bifluorid $10^{\circledR}$ varnish reduced volume loss significantly during the cyclic erosion protocol (90 and $38 \%$ respectively, $\mathrm{p}<0.05$ ) whereas $\mathrm{NaF}$ and $\mathrm{SnF}_{2}$ solutions did not. After 9 cycles of erosion-abrasion, the $\mathrm{NaF}$ and Bifluorid $10{ }^{\circledR}$ were the only groups that showed significantly less volume loss (38 and 50\% respectively, $\mathrm{p}<0.05$ ) compared to the control. In conclusion, the potential for highly concentrated fluoride preparations to provide long-term protection against erosion-abrasion remains uncertain.

University of Oslo.

\section{1 \\ Erosive Effect of Acidic Fluoride Solutions on Enamel in vitro \\ C. Hjortsjö*, G. Jonski, A. Young, E. Saxegaard \\ carl.hjortsjo@odont.uio.no \\ Faculty of Odontology, University of Oslo, Oslo, Norway}

Acidic fluoride solutions have been shown to exhibit erosioninhibiting properties on dental enamel. The aim of this study was to investigate the possible erosive effect of three acidic fluoride solutions on enamel in vitro. The following solutions were tested: $0.2 \%$ hydrofluoric acid (HF, $0.1 \mathrm{~mol} / \mathrm{l} \mathrm{F}^{-}, \mathrm{pH} 3.02$ ), 3.9\% stannous fluoride $\left(\mathrm{SnF}_{2}, 0.5 \mathrm{~mol} / \mathrm{l} \mathrm{F}^{-}, \mathrm{pH} 2.65\right), 1.6 \%$ titanium tetrafluoride $\left(\mathrm{TiF}_{4}, 0.5 \mathrm{~mol} / \mathrm{l} \mathrm{F}^{-}, \mathrm{pH} 1.42\right)$, and $1.8 \%$ citric acid ( $\left.\mathrm{pH} 2.27\right)$ as a control. A total of 24 human third molar enamel specimens were randomly divided into 4 groups (6 specimen/solution). Enamel surfaces were ground flat by wet grinding with a grit 2500 abrasive paper and half of each flat surface was isolated using a A-silicon impression material. The enamel surfaces were exposed to $20 \mathrm{ml}$ of the test solutions for ten minutes in plastic containers. The isolation material was removed. and changes in the enamel surfaces were analysed by LED blue light profilometry $(\lambda=470 \mathrm{~nm}, 50 \times$ magnification). The specimen area analysed included both treated and untreated ground enamel, a total area of $254.64 \times 190.90 \mu \mathrm{m}^{2}$ for each sample. $\Delta$ surface height between treated and untreated enamel was calculated for each specimen and data were analysed using one-way ANOVA and Tukey's test. The mean etching depths $(\mu \mathrm{m} \pm \mathrm{SD})$ were $0.14( \pm 0.86),-2.00( \pm 0.52),-3.25( \pm 2.91)$ and $5.42( \pm 1.00)$ for, $\mathrm{HF}, \mathrm{SnF}_{2}, \mathrm{TiF}_{4}$ and citric acid respectively. There was a significant difference between the control and the fluorides, and between the effect of $\mathrm{HF}$ and $\mathrm{TiF}_{4}(\mathrm{p}<0.05)$. In conclusion, all the acidic fluoride solutions etched enamel significantly less than the citric acid control solution. Both $\mathrm{SnF}_{2}$ and $\mathrm{TiF}_{4}$ treatments re- 
sulted in an average deposition on the enamel surface, while HF showed an average etching effect close to zero.

University grants.

\section{2 \\ The Influence of an Antacid on Intraoral pH after Erosive Challenge}

M.J. Altenburger ${ }^{\mathrm{a}, *}$, N. Bärsch ${ }^{\mathrm{b}}$, K.-T. Wrbas ${ }^{\mathrm{a}}$, E. Hellwig $^{\mathrm{a}}$

markus.altenburger@uniklinik-freiburg.de

aDepartment of Operative Dentistry and Periodontology,

Dental School and Hospital, University Medical Center Freiburg,

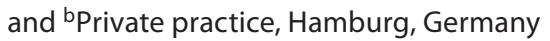

It is well known that strong acids dissolve the surface of enamel and dentin. This chronic exposure to strong acids can cause alterations of the oral mucosa. Reasons for the oral cavity to acidic exposure are e.g. nutrition, drugs or reflux diseases. Most therapy approaches deal with the prevention of acidic exposure or with the modification of the $\mathrm{pH}$ of nutrition or drugs. However the oral cavity can not always be prevented from acidic attacks like during vomitus caused by anorexia. Therefore the approach of the present study was to test an antacid containing aluminium hydroxide and magnesium hydroxide to raise or to neutralize the oral $\mathrm{pH}$ during acidic attacks. To simulate acidic attacks 5 healthy subjects drank $200 \mathrm{ml}$ Sprite light over a period of 1 min every $3 \mathrm{~h}$ for 2 days. In the reference period subjects washed their mouths for $30 \mathrm{~s}$ with water, in the test group with $10 \mathrm{ml}$ antacid containing $900 \mathrm{mg} \mathrm{AlOH}$ and $600 \mathrm{mg} \mathrm{MgOH}$. The teeth of the subjects were protected with splints. Oral $\mathrm{pH}$ was logged using a calibrated $\mathrm{pH}$ probe placed in the anterior part of the palate. Elapsed time until $\mathrm{pH}$ reached $\mathrm{pH}$ $4.5,5.5$ and 6.5 was measured after every intake and statistically analyzed for differences between the two treatment groups using the Mann-Whitney test. Before and directly after the intake of the soft drink $\mathrm{pH}$ values were not significantly different between the experimental ( $\mathrm{pH} 7.3$; $\mathrm{SD} \pm 0.92 / \mathrm{pH} 3.7 ; \mathrm{SD} \pm 0.84)$ and the control group (pH 7.2; $\mathrm{SD} \pm 0.74 / \mathrm{pH} 3.7 ; \mathrm{SD} \pm 1.25$ ). Elapsed times until $\mathrm{pH}$ values reached 4.5 were not different (84 s). However elapsed time (seconds) until the oral $\mathrm{pH}$ reached values of 5.5/6.5 were significantly shorter when the antacid was used (88/SD \pm 21$) /(114 / \mathrm{SD}$ \pm 74 ) in comparison to 134 (SD \pm 76$) / 683$ (SD \pm 926$)$ (both: $\mathrm{p}<$ $0.001)$ in the control group. It can be concluded that rinsing an antacid might be helpful to prevent enamel and dentin from erosion.

The study was not supported financially.

\section{3 \\ Dental Erosive/Abrasive Lesions in Adult People in Belarus \\ N.A. Yudina, D.V. Shabunko* \\ shabun@mail.ru \\ Belarusian Medical Academy for Postgraduate Education, Minsk, Belarus}

Dental erosion/abrasive lesions is defined as a loss of tooth substance by chemical processes not involving bacteria. Erosions my be caused by a variety of extrinsic and intrinsic factors. The aim of this study was to determine of prevalence dental erosion/ abrasive lesions. Four calibrated dentists evaluated dental status of 766 patients of different region of Belarus. The were recorded 35-44 adult people. The prevalence of dental erosion lesions amounted to $5 \%$, abrasive lesions amounted to $14 \%$. These results have suggested that of prevalence dental erosion/abrasive lesions is high.

From own funds.

\section{4 \\ The Use of Calcium Glycerophosphate and Fluoride Varnish in the Protection against Enamel Erosion \\ T.S. Carvalho a, d, * M. Bonecker ${ }^{\text {a }, ~ M . A . R . ~ B u z a l a f ~}{ }^{\text {, F.C. Sampaio }}{ }^{c}$, A. Lussi $^{\mathrm{d}}$ \\ thiagosaads@hotmail.com \\ aDepartment of Pediatric Dentistry, Faculdade de Odontologia da USP, b Department of Biological Science, Faculdade de Odontologia de Bauru, and 'Department of Clinical and Social Dentistry, Universidade Federal da Paraíba, Brazil; d Department of Operative, Preventive and Paediatric Dentistry, University of Bern, Switzerland}

This study aimed at analyzing (1) the amount of loosely (LBF) and firmly bound fluoride (FBF) to enamel and (2) its protection against erosion after calcium glycerophosphate (CaGP) varnish application. (1) 60 human molars had their buccal surface sequentially ground and polished and were randomly distributed into 4 groups ( $n=15$ ), then cut in half; one half served as control (no varnish) and the other received a layer of varnish: (i) NaF varnish (2.26\%F); (ii) $\mathrm{NaF} / \mathrm{CaF}_{2}$ (5.63\%F) varnish; (iii) $1 \% \mathrm{CaGP}+\mathrm{F}$ (5.63\%) varnish; (iv) $5 \% \mathrm{CaGP}+\mathrm{F}(5.63 \%)$ varnish. The varnish was removed after $4 \mathrm{~h}$. The LBF (KOH-soluble) and FBF were analyzed using fluoride electrode. For FBF, the enamel had two layers of $50 \mu \mathrm{m}$ individually ground, dissolved in $\mathrm{HCl}(0.5 \mathrm{M})$, then treated with TISABII; $20 \mathrm{~g} / \mathrm{l} \mathrm{NaOH}$. (2) 108 human molars had their buccal surface sequentially ground and polished, suffered initial erosion challenge ( $90 \mathrm{~s}$ Citric acid; $0.03 \mathrm{M} ; \mathrm{pH}=3.6$ ), then randomly distributed into 6 groups: varnishes (i); (ii); (iii); (iv); (v) no varnish; (vi) base varnish (no F/CaGP). The varnish was applied and removed after $4 \mathrm{~h}$. The samples were alternately demineralized (90 s, Citric acid; $0.03 \mathrm{M} ; \mathrm{pH}=3.6)$ and remineralized (4 h, 1:1 mixture of human and artificial saliv ${ }^{\mathrm{a}}$, once a day, for two more days. Knoop microhardness (KHN) measured enamel softening. (1) Control samples showed significantly less LBF and FBF than varnish groups. Varnish (iii) showed significantly more LBF $\left(0.70 \mu \mathrm{g} \mathrm{F} / \mathrm{mm}^{2}\right)$ and $\mathrm{FBF}$ (553.8 and $276.1 \mathrm{mg} \mathrm{F} / \mathrm{kg}$ enamel, for the outer and inner enamel layer, respectively) $(\mathrm{p}<0.05)$ than other varnishes. (2) KHN decreased in all samples, but varnish (i) suffered significantly less hardness loss $(-75.2 \mathrm{KHN})$ than group (v) $(-103.8 \mathrm{KHN})(\mathrm{p}<0.001)$. CaGP varnishes (iii, iv) were statistically similar to group (v). The addition of CaGP caused an increase in LBF and FBF, but it did not protect enamel against softening from citric acid.

CNPq (201901/2009-9). 


\section{5}

Enamel Erosion Depths Measured on Impressions by a White Light Interferometer

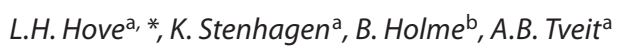

I.h.hove@odont.uio.no

${ }^{a}$ Faculty of Dentistry, University of Oslo, and ${ }^{\text {bSINTEF Materials }}$

and Chemistry, Oslo, Norway

There is a need for clinical studies in order to evaluate progression and prevention of dental erosion. Analysis of erosion depths on impressions may enable in vivo measurements. The aim of this study was to investigate if measurements of the step heights on an impression of eroded enamel surfaces, may give reliable values of the etching depth measured directly on the enamel. Twelve human enamel specimens were made with one circular amalgam reference surface in each, mounted on an epoxy block and ground flat. Baseline images were taken by a white light interferometer (WLI) followed by a 12 min etch by $0.01 \mathrm{M} \mathrm{HCl}, \mathrm{pH}$ 2.2. The mean etch depth on each specimen was measured after three subsequent measurements, both on the specimen surfaces and on the silicone polymer impressions of the surfaces. Paired samples t test was used when comparing the precision of measurements on enamel versus on replica. The mean lesion depths after $12 \mathrm{~min}$ etch measured on the 12 enamel surfaces and replicas were 9.90 and $10.64 \mu \mathrm{m}$, respectively. The direct and indirect measurements correlated well $\left(r^{2}=0.95\right)$ with a $7 \%$ higher mean etch depth measured on the impressions $(p<0.05)$. The reliability of the measurements on enamel and impression expressed by Intra Class correlation (ICC, a 2-way mixed effect model that measures the reliability of repeated measurements by one instrument) were 0.98 and 0.99 , respectively. The average relative precisions of the three repeated measurements on the twelve tooth and impression surfaces were $0.5 \pm 0.09$ and $0.1 \pm 0.02 \%$ and the accuracies were 0.4 and $7 \%$, respectively. In conclusion WLI gave reliable but slightly higher etch depth measurements on impressions compared to enamel surfaces.

Faculty of Dentistry, University of Oslo, Norway.

\section{6}

The Influence of Sour Foodstuffs on the pH Range in the Oral Cavity

M. Decker*, S. Kneist, H. Kuepper, N. Reinhoefer

Mike.Decker@med.uni-jena.de

Center of Telemetry, Center of Dentistry, Clinic for Prosthetic

Dentistry and Dental Materials, University of Jena, Germany

Aim: The aim of the present study was to quantify erosive influences of drops containing different concentrations of citric acid (CCA). Methods: Three methods of $\mathrm{pH}$ measurements were used to monitor $\mathrm{pH}$ values of saliva during drop consumption $(0.5$ to $2.0 \% \mathrm{CCA}$ ). Extraoral (collected saliva) and intraoral $\mathrm{pH}$ levels were evaluated by normal glass electrode (each minute of consumption to $20 \mathrm{~min}$ ). Individual denture including miniature glass electrodes were used for telemetric $\mathrm{pH}$ measurements. Furthermore, bovine enamel specimen with or without 24 -hour in situ pellicle (pell. vs. no pell.) were eroded by drops (0.5 vs. $2.0 \%$ CCA) in different conditions (in vitro vs. in situ). Analyses of microhardness (Vickers, $\Delta \mathrm{HV}$ ) and scanning electron micrographs (SEM) of enamel surfaces followed test procedure. Student $t$ tests were used for statistics. Results: Extraoral and simple intraoral $\mathrm{pH}$ measurements cannot describe complex dissolving processes. Intraoral-telemetric $\mathrm{pH}$ measurements were more valid to elucidate variations of intraoral $\mathrm{pH}$ values during drops consumption. In vitro aqueous solutions of drops ( $15 \mathrm{ml} / \mathrm{drops})$ caused a loss in hardness of enamel without pellicle $(2.0 \% \mathrm{CCA} \rightarrow \Delta \mathrm{HV}=152.6 \mathrm{vs}$. $0.5 \% \mathrm{CCA} \rightarrow \Delta \mathrm{HV}=59.1)$. A 24 -h-in-situ-pellicle reduced these erosive impacts $(2.0 \% \mathrm{CCA} \rightarrow \Delta \mathrm{HV}=55.8$ vs. $0.5 \% \mathrm{CCA} \rightarrow \Delta \mathrm{HV}$ $=4.9)$. In situ effects by pellicles were also dependent of CCA $(0.5 \%$ CCA $\rightarrow \Delta \mathrm{HV}$ no pell. $=6.4$ vs. pell. $=5.4 / 2.0 \% \mathrm{CCA} \rightarrow \Delta \mathrm{HV}$ no pell. $=72.2$ vs. pell. $=64.6)$. Differences were significant between groups of different CCA ( $\mathrm{p} \leq 0.001)$. Results of microhardness test confirmed former telemetric results, which showed that drops with $2.0 \%$ CCA have an erosive potential concerning criteria 'toothfriendly' (40 $\mu \mathrm{mol} / \mathrm{l}\left[\mathrm{H}^{+}\right]$after $\left.15 \mathrm{~min}\right)$. Accessory SEM confirmed that erosions of enamel were dependent on concentration of citric acid and also showed a limited resistance of salivary pellicles against citric acid. Conclusion: Micromorphological analyses showed that intraoral-telemetric $\mathrm{pH}$ measurements were able to evaluate erosive potentials of sour foodstuffs.

\section{7 \\ The Effect of Therapeutic Products in Combination on Prevention of Tooth Surface Loss \\ F. Ali*, K.J. Toumba, M.S. Duggal \\ m.s.duggal@leeds.ac.uk \\ Department of Paediatric Dentistry, Leeds Dental Institute, Leeds, UK}

Aim: To study the effect of specialised fluoride toothpastes, mouthrinses and other remineralising agents specifically marketed for protection against sensitivity and/or erosion in combination on the surface loss of bovine enamel in vitro. Methods: 60 enamel specimens, standardised for hardness, were mounted in acrylic blocks. They were divided into four experimental groups of 15 samples each: 1,400 ppm F as AmF followed by a F and stannous chloride containing rinse 2 times/day (Elmex TP plus MR); 1,450 ppm $\mathrm{F}$ as $\mathrm{NaF}$ and $\mathrm{NaF} 450$ ppm F mouthrinse 2 times/day (PronamelTP plus MR); 1,450 ppm F as NaF + GC tooth mousse once per day (Pronamel TP plus TM) and 0 ppm F control. pH cycling was achieved using citric acid (0.3\%, pH 3.6) for 2 min 5 times/day followed by $1 \mathrm{~h}$ in artificial saliva ( $\mathrm{pH}$ 6.8). Slabs were subjected to 2 min brushing abrasion twice per day with 1:3 slurry of toothpaste/saliva at $300 \mathrm{~g}$ load. At all other times the samples were incubated in artificial saliva. Enamel loss was assessed by profilometry after 7, 14, 21 and 28 days. Results: Normality was checked and data analysed by One-way ANOVA and Bonferroni test. Significantly less surface loss $(\mu \mathrm{m})$ was observed $(\mathrm{p}<0.005)$ with Elmex TP plus MR (0.40 \pm 0.23$)$ and Pronamel TP plus MR $(0.60 \pm 0.28)$ compared with Pronamel TP plus TM $(2.56 \pm 1.61)$ and 0 ppm F control $(1.87 \pm 1.84)$. Conclusions: In this model a 
combination of specialised toothpastes formulated to protect against erosion in combination with mouthrinses significantly decreased the effect of erosion and toothbrushing abrasion. We feel that a regimen comprising a combination of anti-erosion products might be more beneficial in providing protection against such a challenge.

\section{8}

An Electron Microscopic Study of Enamel Treated with Hydrofluoric Acid

\author{
A. Young ${ }^{\mathrm{a}, *}$, C. Hjortsjö $^{\mathrm{a}}$, L. Berthold ${ }^{\mathrm{b}}$, A. Cismak ${ }^{\mathrm{b}}$, M. Petzold $^{\mathrm{b}}$ \\ a.y.vik@odont.uio.no \\ aFaculty of Dentistry, University of Oslo, Oslo, Norway; \\ ${ }^{b}$ Fraunhofer Institute for Mechanics of Materials (IWM), \\ Halle (Saale), Germany
}

Previous studies have demonstrated the effectiveness of treating enamel with acidic fluorides as a means of reducing the effects of erosive challenges. The aim of the present in vitro study was to investigate the surface characteristics of dental enamel treated with hydrofluoric acid (HF). Natural surfaces of enamel specimens from extracted human third molars were treated for $10 \mathrm{~min}$ with either native solutions of $\mathrm{HF}(\mathrm{pH} 3)$ or sodium fluoride $(\mathrm{NaF}$, $\mathrm{pH}$ 7) containing both 0.1 and $0.2 \mathrm{~mol} / \mathrm{l} \mathrm{F}^{-}$. Untreated samples functioned as controls. All specimens were examined by SEM/ EDX with respect to surface microstructure variation and fluoridation. For selected specimens, samples were prepared by FIB allowing cross-sectional investigations using TEM and nanospotEDX. Following treatment with HF, SEM/EDX analyses did not show obvious erosive damage but clearly indicated surface fluoridation. Using TEM, an enamel surface microstructure modification to a depth of approximately $200 \mathrm{~nm}$ with a clear boundary to non-affected apatite was revealed. The fluoride content in this layer was above 40 at $\% \mathrm{~F}$ with a F/Ca ratio of roughly 2 indicating the transformation of surface hydroxyapatite into $\mathrm{CaF}_{2}$-like material. In previous studies, isolated fluoride-rich globular precipitates were found if medium-acidulated solutions of $\mathrm{NaF}$ were applied. The atomic composition of the HF-induced $\mathrm{CaF}_{2}$-like materialresembled that of these precipitates. In contrast, no fluoridation effects were found for the $\mathrm{NaF}$ at $\mathrm{pH} 7$ and the controls in this study. These results suggest that the general reaction between fluoride solutions and enamel leading to a formation of $\mathrm{CaF}_{2}$-like material does not significantly depend on the dissociated fluoride cation. On the other hand, the reaction rate for the acidic HFtreatments is higher since the amount of dissolved calcium increases with decreasing $\mathrm{pH}$.

\section{9 \\ Antierosive/Antiabrasive Effects of Toothpastes in Dentine under Continuous Enzymatic Digestion of Demineralised Surface Material}

\author{
J. von Hinckeldey*, C. Ganss, L. Monert, J. Klimek, N. Schlueter \\ Judith.M.von-Hinckeldey@dentist.med.uni-giessen.de \\ Department of Conservative and Preventive Dentistry, Dental \\ Clinic, Justus-Liebig University, Giessen, Germany
}

With continuing action of acids, the demineralised surface material (DSM) prevents the dentine surface from abrasion and modifies the effect of active agents. In the oral cavity, however, DSM probably does not persist due to the action of proteolytic enzymes. The study aims to investigate the effects of various oral hygiene products mimicking intraoral conditions by the presence of collagenase in the storage solution. For 10 days, dentine specimens ( $\mathrm{n}=$ 18 per group) were demineralised (citric acid, $6 \times 2 \mathrm{~min} /$ day; $\mathrm{pH}$ 2.3) and intermittently stored in collagenase-mineral-salt solution. In experiment 1 (E1), specimens were exposed to toothpaste-slurries for $2 \times 2 \mathrm{~min} /$ day. In experiment 2 (E2), specimens were brushed for $15 \mathrm{~s}$ with $2 \mathrm{~N}$ during the 2 min slurry immersion time. Toothpastes were eight $\mathrm{NaF}$ toothpastes (1,450 ppm F), four special anti-erosive formulations (two F toothpastes: 1,450 ppm F as NaF/ $\mathrm{KNO}_{3}$ and 1,450 ppm $\mathrm{F}$ as NaF/hydroxyapatite; two F-free toothpastes: zinc-carbonate-hydroxyapatite and Chitosan), and two Sncontaining products (3,000 (gel) and 3,500 ppm Sn). Control groups in E1 were erosion only and $\mathrm{Sn} / \mathrm{F}$ mouthrinse; in E2 brushing with placebo and immersion in a Sn/F mouthrinse after placebo brushing. Substance loss was quantified profilometrically. In both experiments, all products, except both F-free and one $\mathrm{NaF}$ toothpaste (E2: additionally one special product) reduced substance loss compared to negative control (E1: $\mathrm{p}<0.01, \mathrm{E} 2$ : $\mathrm{p}<0.001$ ). Small differences between the toothpastes were found; only the 3,000 ppm Sn gel was significantly more effective than all other products in $\mathrm{E} 1$ $(\mathrm{p}<0.05)$ and, except for one NaF toothpaste, in E2 ( $<<0.01)$. Generally, brushing did not increase substance loss. Even after the continuous DSM digestion and under severe erosive conditions, most $\mathrm{F}$ toothpastes reduced erosive and erosive/abrasive substance loss indicating promising perspectives for clinical use.

The study was funded by the Justus-Liebig University Giessen.

\section{0 \\ Assessment of Erosion Using Optical Coherence Tomography on Polished and Natural Enamel Surfaces in situ \\ H.P. Chew ${ }^{\mathrm{a}, *}$, C.M. Zakian ${ }^{\mathrm{a}}$, S.B. Jones ${ }^{\mathrm{b}}$, I.A. Pretty ${ }^{\mathrm{a}}$, N.X. West ${ }^{\mathrm{b}}$, chewhp33@gmail.com \\ aDental Health Unit, The University of Manchester, and ${ }^{b}$ School of Oral and Dental Sciences, University of Bristol, Bristol, UK}

Increased porosity in early eroded enamel, before surface loss, can be quantified with optical coherence tomography (OCT) in vitro. Clinically the erosion rate is likely to be slower due to the 
protective effect of saliva and pellicle. The aim of this study was to investigate the sensitivity of OCT in detecting early erosion in situ and validating it against surface microhardness. 20 volunteers wore a mandibular appliance with two embedded human enamel samples, one with a natural surface (Ns) and the other with a polished surface (Ps) for 5 days. Each day the participants wore the appliances from 09.00 to 16.00 and swished a total of $250 \mathrm{ml}$ orange juice around their mouth for $10 \mathrm{~min}$ at three separate intervals. 3D OCT images and microindents with a Knoop microindenter were made at baseline and at the end of each day. Percentage of surface microhardness change (\%SMC) and decay of backscattered light (At) of OCT were analysed. Multiple regression analysis of erosion interval with \%SMC and At showed that both surface microhardness and OCT detected significant erosion-interval related changes on both natural and polished surfaces with the following $\mathrm{R}^{2}$ value $\left(\% \mathrm{SMC} \mathrm{R}^{2}{ }_{\mathrm{Ns}}=0.19, \mathrm{p}<0.001\right.$; $\mathrm{R}^{2}{ }_{\mathrm{Ps}}=0.60, \mathrm{p}<0.001$ and At $\mathrm{R}^{2}{ }_{\mathrm{Ns}}=0.08, \mathrm{p}=0.01 ; \mathrm{R}^{2}{ }_{\mathrm{Ps}}=0.24$, $\mathrm{p}<0.001)$. There was also significant correlation between \%SMC and At for both Ns and Ps $(\mathrm{p}<0.001)$ with Pearson correlation coefficient of 0.351 and 0.572 respectively. Paired t tests showed significant differences from baseline $(\mathrm{p}<0.05)$ at 30 minutes of erosion onwards for both methods and surfaces. At 30-minute erosion, $\% \mathrm{SMC}_{\mathrm{Nc}}=22 \pm 8$ (mean $\left.\pm \mathrm{SE}\right)(\mathrm{p}=0.009)$ and $\% \mathrm{SMC}_{\mathrm{Ps}}=54 \pm 3(\mathrm{p}<0.001)$ and $\mathrm{At}_{\mathrm{Ns}}=0.05 \pm 0.02(\mathrm{p}=0.005)$ and $\mathrm{At}_{\mathrm{Ps}}=0.13 \pm 0.03(\mathrm{p}=0.001)$. It can be concluded that OCT can detect early erosion on both polished and natural enamel surfaces in situ.

This research was supported by Colgate Palmolive.

\section{1 \\ The Abrasive Effect of a Pig's Tongue on Tooth Tissue Loss under Erosive Conditions}

\section{J. Wheeler*, E.L. Macdonald, S.B. Jones, N.X. West}

\section{s.b.jones@bristol.ac.uk}

School of Oral and Dental Sciences, University of Bristol, Bristol, UK

The tongue is a powerful muscle within the oral cavity and is frequently in contact with the tooth surface and therefore has the ability to damage previously softened enamel. Most models that are used to determine the erosive effects of acid on enamel in vitro do not take the abrasive effect of the tongue into consideration. The aim of this study was to use a pig's tongue to identify the tongue's abrasive influence on tooth tissue surface loss in an erosive environment. Human enamel specimens obtained from an ethically approved tissue bank were sectioned and polished flat. Specimens were pre-soaked in either $0.3 \%$ citric acid at $\mathrm{pH} 3.2$ or artificial saliva for $2 \mathrm{~min}$ or no pre-treatment prior to being rubbed with a pig's tongue in the presence of citric acid or artificial saliva for 15 cycles. Each cycle consisted of pre-treatment soak followed by 50 strokes with the tongue. The amount of surface tissue loss was determined using non-contact profilometry. The greatest amount of tooth tissue loss was observed for specimens that had been soaked in acid and rubbed in an acidic environment
$(1.53 \mu \mathrm{m})$, followed by specimens that were soaked in acid and rubbed in artificial saliva $(1.22 \mu \mathrm{m})$. Following pre-soaking in acid, specimens that were rubbed with or without acid were not significantly different $(\mathrm{p}<0.05)$ to each other. Specimens that were soaked in acid without rubbing $(0.31 \mu \mathrm{m})$ or specimens that were rubbed in artificial saliva with no prior acid exposure $(0.45 \mu \mathrm{m})$ were significantly lower. These results show that the tongue can have a significant effect on the amount of tissue loss if the enamel has previously been exposed to acid and should therefore be considered for in vitro erosion models.

This project was funded by the Clinical Trials Unit at the Bristol School of Oral and Dental Sciences, UK.

\section{2 \\ Applying Profilometry to Quantitatively Detect Initial Erosion of Polished and Natural Surface Enamel in situ}

S.B. Jones ${ }^{\mathrm{a},}{ }^{*}$, H.P. Chew ${ }^{\mathrm{b}}$, C.M. Zakian ${ }^{\mathrm{b}}$, R.P. Ellwood ${ }^{\mathrm{b}}$, M. Davies ${ }^{\mathrm{a}}$, N.X. West ${ }^{\mathrm{a}}$

s.b.jones@bristol.ac.uk

aSchool of Oral and Dental Sciences, University of Bristol, Bristol, and bSchool of Dentistry, The University of Manchester, Manchester, UK

The process of enamel erosion commences as soon as hydroxyapatite is exposed to acid. When designing in situ clinical studies it is advantageous to be able to determine erosion at the earliest time point possible. The aim of this study was to determine how early changes to the enamel surface caused by erosion could be significantly quantified in terms of increase to surface roughness (Ra)using both polished and natural surface enamel. Following ethical approval, 20 healthy individuals participated in this study. Each individual wore lower buccal appliances containing one flat, polished specimen and one curved, natural surface specimen. Lower human incisors were used due to the constraints of the measuring instrument necessitating a curved surface to be within $300 \mu \mathrm{m}$ height variation over a $2 \times 1 \mathrm{~mm}$ scanning area. Individuals wore the appliances from 9.00 to 16.00 and swished a total of $250 \mathrm{ml}$ orange juice around their mouth over a $10 \mathrm{~min}$ period at separate intervals, three times a day for a total of five days. The surface of each specimen was scanned using a non-contact profilometer (Proscan2000, Scantron, UK) following each day and mean Ra determined. Both natural and polished surfaces showed a gradual increase in surface roughness over time ranging from $0.184-0.283 \mu \mathrm{m}(\mathrm{R}=0.231, \mathrm{p}<0.001)$ for the natural surface and $0.086-0.168 \mu \mathrm{m}(\mathrm{R}=0.524, \mathrm{p}<0.001)$ for the polished surface. Significant differences $(\mathrm{p}<0.05)$ were observed following four days $(2 \mathrm{~h})$ of acid exposure for the curved specimens and one day (30 $\mathrm{min}$ ) of acid exposure for the polished specimens. This study confirms that it is possible to use profilometry to measure surface changes caused by erosion of natural and polished specimens but a less erosive challenge was required to elicit significant change with the polished samples.

This project was funded by Colgate Palmolive. 


\section{Caries Research}

\section{Session 6 Diagnostics}

\section{3 \\ Reproducibility of the ICDAS-II Two-Digit Coding Method by Undergraduate Students}

A. Jablonski-Momeni ${ }^{\mathrm{a}, *}$, J. Busche ${ }^{\mathrm{a}}$, M. Heinzel-Gutenbrunner ${ }^{\mathrm{a}}$, R. Frankenberger ${ }^{\mathrm{b}}$, K. Pieper $^{\mathrm{a}}$

momeni@staff.uni-marburg.de

${ }^{a}$ Department of Pediatric Dentistry and ${ }^{\text {b Department of }}$

Conservative Dentistry, Philipps University Marburg, Germany

When the International Caries Detection and Assessment System (ICDAS-II) was introduced, a two-number coding system was suggested to identify restorations/sealants with the first digit, followed by the caries code. The aim of this study was to evaluate the reproducibility of the ICDAS-II two-digit coding method applied by undergraduate students. 24 students (3rd year) were trained in a theoretical session in the use of ICDAS-II two-digit method by an experienced examiner (reference examiner). 26 dental arches with extracted teeth were prepared and examined by the reference examiner. Afterwards the students were randomly divided into two groups A and B. In group A each student (A1A12) examined a random selection of 4 jaws. The same sample was examined by the students in group B (B1-12) independently. The teeth were re-examined after three weeks. Prior to the second examinations group A received an additional theoretical training session. Simple kappa values were calculated for intra- and interexaminer reproducibility of the first digit of ICDAS-II (restoration/sealants). Weighted kappa values were calculated for the reproducibility of the second digit (caries code). For the first digit the kappa values for intra-examiner reproducibility were $0.27-$ 0.96 . For the caries code the kappa values for intra-examiner reproducibility were $0.24-0.83$. Kappa values for inter-examiner reproducibility between the students and the reference examiner were $0.02-0.90$ for the restoration code and $0.10-0.72$ for the caries code. The comparison of the intra-examiner reproducibility between group A and B ( $t$ test), showed no significant differences neither for the first digit nor for the caries code $(\mathrm{p}>0.05)$. Use of the ICDAS-II two-digit method by undergraduate students led to a wide range of intra- and inter-examiner reproducibility kappa values. An additional training session did not show any significant improvement of the kappa values.

\section{4 \\ Direct versus Photographic Caries Assessment Using the ICDAS Criteria in Orthodontic Patients

\author{
S.P. Castaño-Duque, M.D. Hernandez-Zambrano*, G. Bautista, \\ I. Mora, J.S. Lara, S. Martignon \\ smartignon@gmail.com \\ Caries Research Unit UNICA, Universidad El Bosque, Bogotá, \\ Colombia
}

Objective: To compare direct visual versus photographic assessment of caries by means of the ICDAS scoring system, in a sample of patients undergoing fixed appliance therapy. Materials and Methods: The previously cleaned teeth of 101 12- to 35-yearold patients with $\geq 1$ year of appliance treatment were visually examined, using a dental light, air and a WHO probe by a trained examiner (inter-examiner kappa $=0.85$ from a previous study). One month later standardised images (Nikon D80 camera, 105 $\mathrm{mm}$ Nikon Macro, twin flash) were viewed on a monitor (Dell Inspiron 530S). Each patient having 28 teeth, 72 sites were examined per mouth: the occlusal surfaces, including all pits ( 2 in premolars; 3 in molars); and the buccal surfaces, including the fissure and cervical third in the lower molars. Five percent of examinations were repeated. Results: The mean age of subjects was $19.9 \pm$ 6.8 years (55\% female; $45 \%$ male). The intra-examiner reliability was almost perfect for both methods (kappa values $>0.81$ ). A total of 2,114 (75\%) teeth were assessed, corresponding to 4,729 examination sites. By visual exam, the sample showed a mean tradi- 
tional DF-S (ICDAS 4-6) of $8.63 \pm 7.95$ (D-S: $0.23 \pm 0.42$; F-S: $8.41 \pm 8$ ), with the D component being all ICDAS4, non-cavitated dentine lesions. When 1-3 ICDAS scores were included this figure increased to $14.93 \pm 8.91$. All subjects had at least one ICDAS1-4 caries lesion present. The visual photographic examination disclosed a significantly higher (McNemar-Bowker test; $\mathrm{p}=0.000)$ mean number of carious sites $(9.12 \pm 2.58)$ compared with the direct examination $(6.52 \pm 1.92)$. Conclusion: The use of photographic images for the assessment of dental caries by means of the ICDAS criteria detected a higher mean number of sites with caries lesions present.

Universidad El Bosque partially funded this project.

\section{5}

Survey of How Relevant ICDAS-II Is to Japanese General Practitioners Who Focus on Preventive Dentistry

S. Sugiyama, b, *, M. Hayashic, S. Fujikib, d, H.Akimoto ${ }^{\mathrm{b}, \mathrm{e}}$

sdcss@pastel.ocn.ne.jp

aSugiyama Dental Clinic, bThe Japan Health Care Dental

Association, 'Department of Restorative Dentistry and

Endodontology, Osaka University Graduate School of Dentistry,

${ }^{\mathrm{d} O o n i s h i ~ D e n t a l ~ C l i n i c, ~ a n d ~}{ }^{\mathrm{e}}$ Medical Journalist, Japan

The purpose of this study was to check how much Japanese general dental practitioners who regard preventive care as important know about ICDAS-II. A questionnaire was sent to 1,206 members of the Japan Health Care Dental Association (JHCDA), which is a group of general practitioners dedicated to modern patient-centered holistic oral health care. JHCDA developed visual materials named_gPhoto Panel_h to depict the categories of caries proposed by ICDAS-II through oral and X-ray photos. This was sent with the questionnaire. A total of 320 (26.6\%) members returned the questionnaire. Among those, 210 (65.6\%) had heard about ICDAS-II, and 184 (57.5\%) had some specific knowledge about it. More than $90 \%$ of those who responded said that Photo Panel is a useful aid when using ICDAS-II in their clinics. An additional questionnaire was sent to the 160 members who had already had Photo Panel, and they were asked about advantages and difficulties of introducing ICDAS-II into their clinic. A total of 100 clinicians returned it. Positive changes in their clinics after introducing ICDAS-II were as follows: more careful observation of tooth surfaces (58.8\%), sharing the idea of various stages of caries amongst members of their clinics (41.2\%), encouraging explanation about caries to patients (41.2\%). On the other hand, the negative comments were that ICDAS-II required proper training to match the ICDAS grades with clinical practice $(68.1 \%)$ and could lengthen treatment time (44.7\%). The response rate was disappointing especially among a group of committed preventive dentistry, but non-responders might reluctant to change established behavior on the basis of newly developed guidelines. In conclusion, greater efforts are needed to persuade Japanese general practitioners of the usefulness of ICDAS-II, but the visual materials are helpful.

This study was supported by The Japan Health Care Dental Association and Osaka University Graduate School of Dentistry.

\section{6 \\ Colour of Molar-Incisor Hypomineralization Opacity and Its Relationship with Increase in Defect Severity: A Cohort Study}

C.M. Costa-Silva ${ }^{\mathrm{b}}$, F. Jeremias ${ }^{\mathrm{a}}$, J.F. Souza ${ }^{\mathrm{a}}$, G. Ambrosano ${ }^{\mathrm{b}}$, L. Santos-Pinto ${ }^{a}{ }^{*}$, A.C.C. Zuanon ${ }^{a}$, R.C.L. Cordeiro ${ }^{a}$, F. Mialhe $^{\mathrm{b}}$

Ispinto@foar.unesp.br

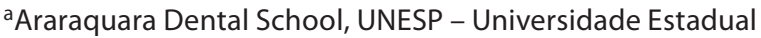

Paulista, Araraquara, and 'Piracicaba Dental School,

UNICAMP - State University of Campinas, Brazil

Predicting the risk of post-eruptive enamel breakdown (PEB) associated with a molar-incisor hypomineralization (MIH) opacity is a difficult but clinically important. The aim of this longitudinal study was to analyze the relationship between the colour of MIH opacities in children at 6-12 years old (baseline) and dental caries, PEB, and demographic variables (children's residential area, years of age and gender). A prospective follow-up was conducted of 147 individuals with MIH for a period of 18 months. Final clinical status (PEB or restoration) was used as a dependent measurement. Enamel opacities were recorded according to colour shades of white, yellow and brown. Relative risk (RR) and confidence intervals (CI) were calculated. To adjust the RR for the effects of covariates, a Poisson regression model was used and the variables that remained at $\mathrm{p}<0.05$ were considered as risk indicators for an increase in the severity of enamel opacity of MIH. After the period of 18 months, 48 teeth presented an increase in $\mathrm{MIH}$ severity, $8.3 \%$ being associated with white opacities, $60.4 \%$ with yellow and $31.3 \%$ with brown opacities. Among the molars, PEB was found more frequently than atypical restorations. Atypical composite resin restorations were found more frequently than PEB among incisors that presented increase in MIH severity. Brown and yellow MIH opacity were at higher risk for PEB and atypical restorations than white ones, even after adjustment for clinical and demographic variables. This result could help clinicians determine a risk based treatment for children with MIH.

FAPESP, CNPq and Fundunesp, Brazil.

\section{7 \\ Assessing Caries Detection by Undergraduate Dental Students}

R.C.L. Cordeiro*, M.B. Diniz, L.M. Lima, C.E. Zago, D. Bussaneli, L. Santos-Pinto

ritacord@foar.unesp.br

Araraquara Dental School, UNESP - Universidade Estadual

Paulista, Araraquara, Brazil

The influence of undergraduate dental students' clinical training in occlusal caries detection using the ICDAS criteria was carried out in two steps: (1) Four dental students without previous experience in caries detection accessed 104 permanent extracted teeth with occlusal caries lesions giving scores for ICDAS criteria, before and after e-learning program. (2) Three years later, experienced students (ES) from step 1 and another four students with- 
out previous experience (NES) but cursing the same level of the first one was introduced to the ICDAS e-learning program and both groups applied this criteria in 102 permanent extracted teeth. All assessments were carried out twice observing a oneweek interval and the teeth were prepared for histological examination. Kappa and intraclass correlation coefficients were used for reproducibility, ANOVA and ROC for a significant change in the ICDAS scores before and after e-learning and ES and NES. Step 1: Correlation between the outcomes and histological scores was moderate ( 0.57 before and 0.61 after e-learning). Reproducibility was high for intra- and inter-examiner ( 0.75 and 0.72 before e-learning) and (0.82 and 0.78 after e-learning). The ROC curve was not significant $(\mathrm{p}=0.10)$. Step 2: Correlation between the outcomes and histological scores was moderate (0.63 to 0.69 for ES and 0.64 to 0.72 for NES). Reproducibility intra-examiner was moderate $(0.48$ to 0.58$)$ and inter-examiner was poor $(0.24$ to $0.40)$. The ROC curve was not significant (0.762) for ES and significant $(\mathrm{p}=0.0001)$ for NES. The e-learning programme did not improve the performance of the visual examination criteria and previous clinical dental experience does not seem to play a significant role in learning ICDAS.

FAPESP (2009/00218-0), Brazil.

\section{8 \\ In vivo Comparison of Methods for Approximal Caries Detection in Primary Molars}

F. Purger ${ }^{\mathrm{a}}$, P.R. Souza ${ }^{\mathrm{b}}$, J.A. Rodrigues ${ }^{\mathrm{c}}$, A. Lussi ${ }^{\mathrm{d}}$, A.B. Vasconcellos ${ }^{\mathrm{b}}$, A. Ribeiro ${ }^{\mathrm{b}, *}$

apoenaribeiro@vm.uff.br

${ }^{a}$ Faculty of Dentistry, Fluminense Federal University, Niterói,

${ }^{b}$ Faculty of Dentistry, Fluminense Federal University,

Nova Friburgo, and 'Faculty of Dentistry, Federal University of Rio Grande do Sul, Porto Alegre, Brazil; dSchool of Dental Medicine, University of Bern, Switzerland

The aim of this study was to compare the performance of different methods in detecting approximal caries lesions in primary molars, in vivo. In four appointments, 209 approximal surfaces were examined: (a) examiner 1 made visual-tactile examination (VT-1) using the Nyvad Criteria; examiner 2 used DIAGNOdent pen 2190 (LFpen; DDp-1) and took bitewings radiographs (BW); (b) one week later: examiner 2 made the DDp-2; (c) another week later: after tooth separation, examiner 1 made VT-2 and examiner 2 made DDp-3; (d) after tooth exfoliation, surfaces were directly examined (DDp-4). Kappa values for examiner 1 and 2 were, respectively, 0.90 and 0.94 . Surfaces were examined by computed microtomography as reference standard $(\mu \mathrm{CT})$. Analyses were based on three diagnostic thresholds, distinguishing between sound tissue and enamel/dentine carious lesion (D1); all sound/enamel and dentine lesions (D2); and sound/non-cavitated and cavitated enamel or dentine lesions (D3). Spearman's correlation scores: between VT-1/VT-2 (0.57), VT-1/BW (0.34), VT-1/ $\mu \mathrm{CT}(0.33), \mathrm{VT}-2 / \mu \mathrm{CT}(0.35), \mathrm{DDp}-3 / \mu \mathrm{CT}$ (0.35). At D1, the highest specificity and sensitivity values were observed for DDp-4 (0.68) and for VT-1 (1.00), respectively. At D2, DDp-4 and BW showed the highest value of sensitivity (0.69) and specificity (1.00) respectively. At D3, DDp-4 showed highest value of sensitivity (0.78) and VT-1, VT-2 and BW the higher scores of specificity (1.00). Accuracy values were higher for DDp-3 at D1 (0.75), DDp4 at D2 (0.78) and D3 (0.87). However, VT-1 showed higher accuracy values than DDp-1 and VT-2 showed similar values to DD-3. It was concluded that visual-tactile examination, using the Nyvad Criteria, showed better results in detecting approximal caries lesions in primary molars than either bitewing radiographs or the LFpen.

We would like to acknowledge the financial support of the FAPERJ and PROPPi/UFF.
109
Assessment of Marginal Defects Adjacent to Restorations Associated with Caries by Optical Coherence Tomography
M. Weuste ${ }^{\mathrm{a},}$, H. Schneider ${ }^{\mathrm{a}}$, B. Fischer ${ }^{\mathrm{b}}$, K. Park $^{\mathrm{a}}$, R. Haak ${ }^{\mathrm{a}}$
malte.weuste@medizin.uni-leipzig.de
aniversity of Leipzig, Department of Conservative Dentistry and Periodontology, Leipzig, and ${ }^{\mathrm{b}}$ Fraunhofer Institute for
Non-Destructive Testing, Dresden Branch, Germany

Objectives: To evaluate optical coherence tomography (OCT) in its ability to detect and assess discolourations and gaps adjacent to enamel margins of composite restorations potentially associated with caries lesions. Eight extracted human molars with 11 carious lesions adjacent to composite restorations were selected from a pool of extracted teeth. Lesions were selected by visual inspection and laser fluorescence readings (DIAGNOdent). The regions of interest were labeled by aligning two lesions per tooth in between two prepared cone-shaped holes. The teeth were imaged by frequency domain OCT [(FD) OCT, $830 \mathrm{~nm}$ ] and the predefined layers were assessed histologically (6.25x magnification) and by microtomography ( $225 \mathrm{kV}$, microfocus X-ray source) for lesion extension and extent of marginal discrepancies of the restorations. Additionally lesions were imaged by microtomography (225 kV-microfocus X-ray source). Signal depth of OCT was measured assuming a refractive index of 1.6. Measurements were assessed statistically ( $\alpha=0.0125$, Wilcoxon test, McNemar test, Bland-Altman plot). Results: Bland-Altman plots of marginal gap width (OCT: 75-888 $\mu \mathrm{m}$, histologically: 59-880 $\mu \mathrm{m}$ ) and depth (OCT: 70-239 $\mu \mathrm{m}$, histologically: 90-224 $\mu \mathrm{m}$ ) did not indicate increased values for either method. The parameters were not significantly different and correlated (width: $\mathrm{p}=0.848, \mathrm{r}=0.96(\mathrm{p}<$ 0.0005); depth: $\mathrm{p}=0.653, \mathrm{r}=0.95$ ( $\mathrm{p}<0.0005)$ ). OCT signals showed no significant differences depending on presence/absence of marginal discoloration ( $\mathrm{p}=0.25$; correlation: $\mathrm{r}=0.516(\mathrm{p}=$ 0.104)). Maximum signal width (OCT: 70-539 $\mu \mathrm{m}$, histologically: 53-515 $\mu \mathrm{m})$ at the cavity wall was significantly increased with OCT $(p=0.001, r=0.88(p<0.0005))$. Depth of the OCT signal varied between 203 and 1,826 $\mu \mathrm{m}$. Conclusion: Marginal discolorations and gaps of composite restorations were detectable with non-invasive imaging by FD-OCT in principle.

Supported by University of Leipzig, Medical Faculty. 


\section{0 \\ Impact of an ICDAS e-Training Programme on Examiner Reliability}

K.W. Neuhaus ${ }^{\mathrm{a}, *}$, J.A. Rodrigues ${ }^{\mathrm{b}}$, F. Maeder $^{\mathrm{a}}$, A. Baltisberger ${ }^{\mathrm{a}}$,

I. Hug ${ }^{\mathrm{a}}$, A. Lussia

klaus.neuhaus@zmk.unibe.ch

aDepartment of Preventive, Restorative and Pediatric Dentistry, University of Bern, Switzerland; bFaculty of Dentistry, Federal

University of Rio Grande do Sul, Brazil

The aim of this study was to compare the effect of different tuition modalities for visual caries diagnostics on intra-and interexaminer reliability. Fifteen dentists with 2-6 years of clinical experience were divided in three groups. Group A received an individual face-to-face teaching lesson of 60-90 min duration with practical demonstration on extracted teeth. Group B was asked to watch the ICDAS e-learning software (www.icdas.org/elearning. html). Group C completed a newly written e-training programme. This programme is partly informational and contains a quiz with more than 120 teeth. Each question of the quiz contains a tooth with a marked point of interest (photographed wet and dry) as well as the corresponding histological section. Immediate feedback of the answer is provided. The e-training programme had to be repeated until a success rate of $80 \%$ was achieved. First, the dentists examined occlusal surfaces of 104 extracted permanent molars, having been provided only basic information on the ICDAS codes. After receiving tuition according to their groups, they repeated the assessment at least one week later. Intra-examiner reliability (Cohen's kappa) and inter-examiner reliabilities before and after tuition (Fleiss' generalized kappa and corresponding 95\% confidence intervals) were calculated. Intra-examiner kappa means were 0.44 (group A), 0.49 (group B) and 0.38 (group C), respectively. Inter-examiner agreement before any teaching was not significantly different in all groups. After tuition, inter-examiner reproducibility in groups $\mathrm{A}$ and $\mathrm{C}$ was not significantly different from each other, but was significantly higher than group B. An improvement in reproducibility could not be detected in group B. However, Fleiss' kappa values were generally low, not exceeding 0.22 . In terms of reproducibility, the newly developed e-training software seems to be comparable to face-to-face teaching.

\section{1 \\ Dentinal Anomalies beneath the Enamel-Dentine Junction Could Explain the Early Onset of Occlusal Caries Lesions}

J. Kühnisch*, K. Bücher, M. Galler, M. Seitz, R. Hickel, K.H. Kunzelmann

jkuehn@dent.med.uni-muenchen.de

Department of Conservative Dentistry and Periodontology,

Maximilians University of Munich, Germany

The phenomenon of hidden caries on occlusal pits and fissures is well-known. Clinically, the carious process develops below a sound-appearing layer of enamel, which may hide a dentinal lesion of even considerable activity and size. As main reason has to be discussed the fissure morphology with a reduced enamel thickness in relation to a plaque retaining fissure pattern. There are no reported studies on the presence of mineralisation disturbances beneath the enamel-dentine junction (EDJ). Therefore, this in vitro study aimed to investigate the EDJ on occlusal surfaces in detail. 204 third molars with clinically sound occlusal fissures or non-cavitated lesions were selected. Prior to the histological investigation each tooth was examined visually (UniViSS) and radiographically (Sidexis, Sirona, Bensheim, Germany). All specimens were non-invasively scanned with the $\mu$ CT40 (Scanco Medical, Basserdorf, Switzerland). Additionally, all teeth were sectioned (Leica-SP1600, Leica Microsystems GmbH, Nussloch, Germany) and each slice was investigated with a stereomicroscopic. The study was approved by the ethics committee of the LMU of Munich. In seven out of 204 third molars (3.4\%) sharpedged radiolucencies were detected below the EDJ in the $\mu \mathrm{CT}$ data set. The detailed comparison with the corresponding histological sites revealed hard tissue defects in dentine as well. Interestingly, beneath these hypoplastic areas in dentin no typical histological caries symptoms were detected ( $\mu \mathrm{CT}$ : no gradient of demineralisation, histology: no demineralisation/discoloration). The visual and radiographical examination showed in all of these cases no signs of caries. It may be concluded that the described defects can be interpreted as dentine anomaly beneath the EDJ whose role in the caries process is not known. Further studies should take this finding into account.

This study was supported by a grant from the German Society of Conservative Dentistry (DGZ-GABA-Wissenschaftsfond, AZ: DGZ-GABA-WF/Bücher 2010-1).

\section{2 \\ Factors Associated with Surgical Intervention for Occlusal Caries}

M. Stacey, M. Hopcraft*

m.hopcraft@unimelb.edu.au

Melbourne Dental School, University of Melbourne, Australia

There is a large variation in the diagnosis and restorative treatment decisions of dentists. The aim of this study was to investigate factors associated with surgical intervention for primary occlusal caries in adult patients. Questionnaires were sent to a random sample of dentists, with 432 useable responses received. High quality colour photos of occlusal surfaces of previously extracted maxillary molars representing the ICDAS-II codes $0-5$ were provided to participants. They were then required to determine treatment options for two different case scenarios, assuming the teeth were second molars in either a 20 - or 40 -year-old patient with good health and good oral hygiene. The study was approved by the University of Melbourne Human Research Ethics Committee. Dentists generally decided to intervene surgically much earlier in the caries process for the 20 -year-old patient compared with the 40 -year-old patient, with $66.1 \%$ of dentists intervening surgically for ICDAS-II code 2 lesions in the 20-year-old patient compared with only $46.3 \%$ for the 40 -year-old patient, whilst the majority (70.8\%) only decided to intervene restoratively for ICDAS-II code 4 lesions in the 40-year-old patient. Logistic regression models showed no clear patterns for decision making across all scenarios, 
however older dentists and those who obtained their dental degree from overseas were more likely to intervene surgically with ICDAS-II codes $1-3$ lesions $(\mathrm{p}<0.05)$, and dentists from rural areas and those working in the public sector were less likely to intervene surgically for these lesions $(\mathrm{p}<0.05)$. Conclusion: Dentists appeared to intervene surgically in caries lesions at a very early stage in the disease process, with the majority placing a restoration for ICDAS-II code 2 lesions.

Melbourne Dental School.

\section{3 \\ Evaluation of Thermal Images under Pulse Heating for Quantification of Sound, De- and Re-Mineralized Enamel}

M. Ando ${ }^{\mathrm{a}, *}$, T. Sakagami $^{\mathrm{c}}$, G. Eckert $^{\mathrm{b}}$, D. Zero ${ }^{\mathrm{a}}$

mando@iupui.edu

andiana University School of Dentistry, Department of Preventive and Community Dentistry, and ${ }^{\mathrm{b}}$ Indiana University School of Medicine, Division of Biostatistics, Indianapolis, USA; 'Kobe University Graduate School of Engineering, Department of Mechanical Engineering, Kobe, Japan

The objective was to evaluate whether the pulse heating thermal images could distinguish among sound, de- and re-mineralized enamel. 60 human extracted permanent anterior teeth were covered with nail varnish except on the labial surface of two $1 \times$ $1 \mathrm{~mm}^{2}$ windows. Specimens were divided into 2 groups and demineralized for 3 or 7 days by a microbial caries model using Streptococcus mutans A32-2. One window was covered. Specimens were further divided into 2 groups and remineralized with 1,100 ppm F as NaF for 7 or 20 days by a $\mathrm{pH}$ cyclic model. The distribution of infrared radiation energy was acquired via infrared camera (113 images/s). A xenon flashlamp was employed as the heat source $(1,600 \times 4 \mathrm{~J}$ and excitation-time: 1/1,200 s). Sound, demineralized, and remineralized enamel areas were analyzed. The temperature descent curve between Ir (intensity of infrared radiation energy) and (elapsed time after pulse heating) was fitted on the logarithmic curve expressed by the following equation: $\mathrm{Ir}=-\mathrm{A} \ln (\mathrm{t})+\mathrm{B}$. Repeated measures ANOVA models were used to compare the coefficient A estimates between groups and treatment periods (sound, demin, remin). 3 days-demin/7 days-remin was removed from the analyses due to lack of observed demineralization. Average \pm Standard-deviation of coefficient A (sound, demin, remin) were: 7 days-demin/7 days-remin: $229 \pm 27,382$ \pm 51, $352 \pm 24,3$ days-demin/20 days-remin: $254 \pm 37,392 \pm$ $70,335 \pm 43$, and 7 days-demin/20 days-remin: $267 \pm 101,432$ $\pm 110,381 \pm 60$. Sound areas had lower $A$ than demin and remin areas $(\mathrm{p}=0.0001)$, and demin areas had higher $\mathrm{A}$ than remin $(\mathrm{p}=$ $0.0001)$. There was no difference among groups for sound period or demineralized period ( $\mathrm{p}>0.20$ ). For remin, 3 days-demin $/ 20$ days-remin was lower than 7 days-demin/20 days-remin $(\mathrm{p}=$ 0.002 ) while 7 days-demin/7 days-remin was not different from 3 days-demin/20 days-remin or 7 days-demin/20 days-remin $(p>0.05)$. These results showed that pulse heating thermal imaging can distinguish among sound, de- and re-mineralized enamel.

Indiana University School of Dentistry.
114

\section{Aspects of Occlusal Red Fluorescence in Relation to ICDAS Scoring in vitro}

D. Inaba ${ }^{\mathrm{a}, *}$, B.-I. Kim ${ }^{\mathrm{b}}$, E. de Josselin de Jong ${ }^{\mathrm{c}-\mathrm{e}}$, M.H. van der Veen ${ }^{\mathrm{d}}$

d.inaba001@gmail.com

aDepartment of Oral Health and Development Sciences, Iwate

Medical University, Morioka, Japan; ${ }^{b}$ Department of Preventive

Dentistry and Public Oral Health, College of Dentistry, Yonsei

University, Seoul, Korea; 'Inspektor Research Systems BV, and

${ }^{d}$ Academic Centre for Dentistry Amsterdam (ACTA), Amsterdam, The Netherlands; ' School of Dental Sciences, University of

Liverpool, Liverpool, UK

We previously demonstrated that sub-surface caries (bacterial invasion zone) can be detected quantitatively based on the porphyrin-induced red fluorescence images captured by a novel quantitative light-induced fluorescent system (QLF-D, Inspektor Research Systems BV, Amsterdam). The aim of this study was to examine the aspects of occlusal red fluorescence of human molars in relation to the ICDAS scoring in vitro. Occlusal fluorescence images of 20 extracted human molars ranging from $0-6$ in the ICDAS detection codes were collected by the QLF-D system fitted with the red-color specific optical filters and 405-nm blue light excitation spectra. Two parameters were employed to assess red fluorescence levels: (1) parameter S (R/G), defined as the average $\mathrm{R} / \mathrm{G}$ value of the tooth, and (2) $\Delta \mathrm{S}$, defined as the averaged ratio of $R / G$ in red area with respect to $R / G$ ratio in sound area. The $S$ $(\mathrm{R} / \mathrm{G})$ values were distributed from 0.85 to 1.28 corresponding to the ICDAS scoring, and correlated significantly to the ICDAS codes with $r=0.60$ (Spearman rank correlation; $p=0.0048$ ), while $\mathrm{r}=0.43$ for the $\Delta \mathrm{S}$ values at $\mathrm{p}=0.06$. Due to the logistic regression analysis, the parameter $S$ indicated sensitivity of 0.78 and specificity of 0.73 to differentiate the codes 3 and 4 with a cut-off value of 1.06. In conclusion, it was suggested that the red fluorescent parameter S (R/G) measured from red fluorescent images by QLF$\mathrm{D}$ have good agreement with the ICDAS codes.

\section{5 \\ Use of Magnetic Resonance Microscopy in Assessment of Caries and Dental Pulp Integrity \\ K. Cankar ${ }^{\mathrm{a}, *}$, L. Nemeth $^{\mathrm{b}}$, J. Vidmar ${ }^{\mathrm{a}, \mathrm{c}}$, I. Serša ${ }^{\mathrm{c}}$ \\ ksenija.cankar@mf.uni-lj.si \\ anstitute of Physiology, Medical Faculty, University of Ljubljana, ${ }^{b}$ Department of Dental Diseases, University Medical Centre Ljubljana, and 'Jožef Stefan Institute, Solid State Physics \\ Department, Ljubljana, Slovenia}

Magnetic resonance imaging (MRI) was seldom used in dental caries studies despite its numerous advantages. No study so far has associated MRI of caries lesions with dentine-pulp complex response encoded in ICDAS scores. MR microscopy of decayed extracted human teeth (10 molars and 2 premolars) with positive electric vitality tests was performed in $2.35 \mathrm{~T}$ horizontal bore superconducting magnet. After ICDAS scores were determined, the 
teeth were extracted and immediately placed in saline solution to prevent drying out. Before the MRI scan, the teeth were coated by a dental impression material to prevent pulp desiccation and to visualize their outline. The samples were imaged by the $3 \mathrm{D} \mathrm{T}_{1}$ weighted spin-echo and by the 3D DWI method. Volume rendered images were calculated from the $\mathrm{T}_{1}$-weighted images while ADC maps were calculated from four DW images at different $b$ values. Volume rendering enabled a 3D analysis of the pulp chamber anatomy from an arbitrary view point, detection of affected root canals as well as the assessment of the distance between caries lesions and the pulp. ADC mapping of the decayed teeth enabled quantification of the pulp tissue integrity and differentiation between an intact $\left(1.2 \pm 0.1 \times 10^{-9} \mathrm{~m}^{2} / \mathrm{s}\right)$ and infected $(0.9$ $\pm 0.1 \times 10^{-9} \mathrm{~m}^{2} / \mathrm{s}$ ) regions of the pulp. A positive correlation was found between the average ADC values of the infected dental pulp and the corresponding ICDAS scores $(r=0.83, \mathrm{p}<0.01)$. We conclude that MR microscopy enables detection of approximal lesions and occult caries in dentin. The pathologies are associated with the MRI signal rise in $\mathrm{T}_{1}$-weighted images of the demineralised dental hard tissues and the decrease of ADC values in the infected regions of the pulp.

Supported by Slovenian Ministry of Science and Education (No. PO-510-381).

\section{6 \\ Development of Caries Indices Using Quantitative Light-Induced Fluorescence (QLF) in vivo}

M.R. Alammari, P.W. Smith*, E. de Josselin de Jong, S.M. Higham

manal@liverpool.ac.uk

School of Dentistry, University of Liverpool, Liverpool, UK

The prevalence of dental caries although declining, still remains widespread. Clinical assessment of early caries is based on qualitative methods relying on dentist's subjective interpretation. An objective and well-defined means of classifying lesions by QLF does not exist. The study aimed to develop and validate a clinically applicable index for QLF relating green fluorescence loss $(\Delta \mathrm{F}$ and $\Delta \mathrm{Q})$ and extent of red fluorescence $(\Delta \mathrm{R})$ to ICDAS II scores for occlusal, buccal and lingual surfaces (OBL). This intended to categorise QLF values as index scores and validate in vivo a QLF caries index previously developed in vitro. Ethics approval was obtained. 83 subjects attending an outpatient clinic were recruited yielding 350 posterior teeth. ICDAS II scoring was used. Images of (OBL) surfaces for each tooth using different systems (white-light digital camera and QLF camera were taken to show the difference in the appearance of different stages of dental caries. Periapical radiographs (part of the usual clinical procedure) and histology were employed. QLF values $\Delta \mathrm{F}, \Delta \mathrm{Q}$ and $\Delta \mathrm{R}$ correlated significantly with ICDAS II scores 1,2 , and 3 (p $<0.001) . \Delta \mathrm{F}$, $\Delta \mathrm{Q}$ and ICDAS II each correlated significantly with histology $(0.780,0.700$ and 0.770 respectively). There was a significant difference in $\Delta \mathrm{F}$ for different histological scores $(\mathrm{p}=0.002)$ which provided necessary separation between scores assigned to the histological extent of caries and changes in $\Delta \mathrm{F}$. Sensitivity and specificity for the QLF index compared with histology were $0.89,0.81$ respectively. Results also demonstrated that radiographs performed poorly as a method to detect early dental caries. It is anticipated that QLF may enhance the identification of early demineralisation and will be a valuable tool in clinical practice and reduce the exposure of patients to ionising radiation.

International scholarship from King Abdulaziz University, Kingdom of Saudi Arabia.

\section{7 \\ Depth of Proximal Caries Lesions in Primary Molars Assessed by MicroCT \\ V.M. Soviero ${ }^{\mathrm{a}, *}$, S.C. Leal ${ }^{\mathrm{b}}$, R. Carvalho ${ }^{\mathrm{c}}$, R.B. Azevedoc \\ verasoviero@gmail.com \\ aDepartment of Preventive and Community Dentistry, Universidade do Estado do Rio de Janeiro, bepartment of Dentistry, Universidade de Brasília, and 'Department of Genetics and Morphology, Universidade de Brasília, Brazil}

This study aimed to assess the depth of proximal caries lesions in primary molars using MicroCT in comparison to visual examination and bite-wing having histological examination as gold standard. Twenty five primary molars (48 proximal surfaces) with no restorations and no enamel defects were selected for the study. Two calibrated examiners conducted the examinations independently. Disagreements were reexamined to achieve consensus. Proximal surfaces were visually scored using ICDAS as: $0=$ sound; 1 = enamel caries after drying; 2 = enamel caries under moisture; 3 = enamel breakdown; 4 = shadow in dentin; 5 = cavity. Bitewing, MicroCT and histological examination were based on the following scores: $0=$ sound; $1=$ outer enamel caries; $2=$ inner enamel caries; 3 = not spread dentin caries; 4 = outer dentin caries; 5 = inner dentin caries. At MicroCT, axial and sagittal sections were examined. For histological validation, sections of around $400 \mu \mathrm{m}$ thickness were analysed at stereomicroscope with 10x magnification. Inter-examiner agreement ranged from 0.82 to 0.90 kappa coefficient. According to the gold standard, the frequency of caries scores was: 9 (18.8\%) sound, 23 (48\%) enamel caries and 16 (33.3\%) dentin caries. The highest correlation with gold standard was observed for MicroCT (rs 0.88). Sensitivity (SE), specificity (SP), accuracy (AC), positive (PPV) and negative predictive value (NPV) were calculated for two diagnostic thresholds: enamel (E1) and dentin caries (D1). At both E1 and D1, MicroCT achieved the highest values for SE, SP, AC, PPV and NPV $(0.92 ; 0.67 ; 0.88 ; 0.92 ; 0.67$ and $1 ; 0.97 ; 0.98 ; 0.94 ; 1$; respectively). Disagreements between MicroCT and the gold standard must be analysed carefully taking into consideration that sectioning may wear a considerable amount of dental tissue. MicroCT showed high correlation with gold standard being a promising method to assess the depth of caries lesions in in vitro studies.

INCT Nanobiotecnologia CNPq. 


\section{8 \\ Evaluation of the Impact of Different Clinical Conditions on Caries Experience Using the Nyvad Criteria}

J. Véronneau a, S. Tikhonova ${ }^{\mathrm{a}, *}$, N. Pustavoitava ${ }^{\mathrm{b}}$

svetlana.tikhonova@mail.mcgill.ca

aFaculty of Dentistry, McGill University, Montreal, Canada;

${ }^{b}$ Department of Therapeutic Dentistry, Belarusian State Medical

University, Minsk, Belarus

This study was designed to evaluate the impact of different clinical conditions on caries diagnosis using the Nyvad criteria. Three volunteer groups (A, B, C) of 11 to 16 years old Belarusian school children were examined twice using the Nyvad criteria under three clinical conditions: (A) two hands versus four hands for examination and data entry, (B) preliminary tooth brushing versus not, (C) standard dental settings versus field. The total number of participants was 148 (49-50 subjects in each group). Examinations were done using the Nyvad criteria by one trained and calibrated dentist in school dental office with mouth mirrors, dental explorers and preliminary tooth brushing (except group B, no brushing). Examinations for clinical conditions $\mathrm{A}$ and $\mathrm{B}$ were done on dental chairs using artificial dental light, suction device and three-in-one syringe. Examinations for clinical condition $\mathrm{C}$ (field settings) were done on school chairs near a window using a dental explorer, mouth mirror with built in light and cotton rolls. Mean DMFS for two hands vs. four hands was 32.86 (SD \pm 18.93 ) and 33.82 (SD \pm 19.67 ), respectively ( $p>0.05$ ). Mean DMFS value of group B was consistently higher with preliminary brushing (29.69 (SD \pm 16.88$)$ ) compared to no brushing (26.12 (SD \pm 14.25$)$, $\mathrm{p}<0.001)$. For the group C mean DMFS was $19.14(\mathrm{SD} \pm 11.84)$ for the patients examined in standard dental settings and 17.38 (SD \pm 12.64 ) for the same patients examined in the field settings ( $p<$ 0.05 ). Active lesions (mean Dactive) were consistently higher for the standard dental settings (7.52 (SD \pm 7.50$)$ ) compared to field settings (4.94 (SD $\pm 7.49, \mathrm{p}<0.001)$ ). In conclusion, dental caries experience estimation in adolescents may be influenced by clinical or field conditions when the Nyvad criteria used for diagnosis.

Funded by Health Research Fund of Quebec, Canada.

\section{9 \\ International Evaluation of Dental Profession Perceptions on Developing a New Instrument in Cariology, the Exprober}

J. Véronneau' ${ }^{\mathrm{a}}$,M. Badea ${ }^{\mathrm{b}}$ * , N. Pustavoitava', L. Rosado ${ }^{\mathrm{d}}$

jacques.veronneau@mcgill.ca

a McGill University, Montreal, Canada; ${ }^{\mathrm{b}}$ Department of Preventive Dentistry, University of Medicine and Pharmacy luliu Hatieganu, Cluj-Napoca, Romania; 'Department of Therapeutic Dentistry, Belarusian State Medical University, Minsk, Belarus; ${ }^{\mathrm{d} D e n t a l}$ Faculty of Catolica Santa Maria University, Arequipa, Peru

Modern dental carious lesion detection and assessment is a major challenge for dental scientific evidence transfer in a clinical setting since this procedure affects several treatment outcomes.
This study was designed to assess the perceptions of different people concerned by the dental caries diagnosis procedure on the relevance of developing a new cariology instrument, the exprober. The unique design of the exprober provides 3-in-1 activities: caries detection, pocket probing and cleaning of light calculus and plaque. Four trained, standardized dentists from four collection sites (two in Europe, one in South and one in North America) coordinated the survey. A total of 292 questionnaires were completed by seven respondent categories: institution dentist clinician, private practice dentist, university cariology professor, dental student, dental hygienist and dental patient. A standardized introduction to the questionnaire outlined the goals of developing the exprober and showed an image of the instrument prototype. Each questionnaire included the same first general section with questions on developing the instrument (invention acceptance) and a second specific section with questions adapted to each respondent type on daily use or impact (planned functions, improvement of cariology transfer, clinical adoption). A total of 25 open-ended questions had four answer choices: 1 = agree; 2 = neutral, $3=$ disagree; $4=$ don't know. Each respondent was asked to review the questionnaire, tick off the answer choice and submit an electronic or hard version of the completed form. Among all the respondents, $85,97,89$ and 93\% (73-99\% range) answered with agree on invention acceptance, on planned functions, on improvement of cariology transfer and on clinical adoption, respectively. European and American sites agreed with a mean proportion of 93 and of $87 \%$, respectively, for all categories of questions. All types of dental respondents had high similar proportion (89-93\% range) supporting the instrument development. The development of a new dental instrument for simultaneous visual-tactile caries/risk/ plaque-control diagnosis appears to be largely supported by all international sites, by all type of dental respondents and for all categories of documented questions.

Funding: McGill Office of Technology Transfer, Montreal, Quebec, Canada.

\section{0 \\ Calibration Study of the Nyvad Clinical Criteria for Scoring Caries Lesion Activity in Minsk (Belarus) \\ N. Pustavoitava, ${ }^{\text {a }}$, B. Nyvad ${ }^{\mathrm{b}}$, V. Baelum ${ }^{\mathrm{b}}$, S. Tikhonova ${ }^{\mathrm{c}}$, V. Machiulskiene ${ }^{\mathrm{d}}$ \\ natallia.pustavoitava@gmail.com \\ aDepartment of Therapeutic Dentistry, Belarusian State Medical University, Minsk, Belarus; ${ }^{b}$ School of Dentistry, Faculty of Health Sciences, University of Aarhus, Denmark; ${ }^{\text {CFaculty }}$ of Dentistry, McGill University, Montreal, Canada; ${ }^{d}$ Faculty of Health Sciences and Faculty of Odontology, Lithuanian University of Health Sciences, Minsk, Lithuania}

Studies using Nyvad criteria have shown that clinical caries lesion activity assessments in adolescent populations may be reliable and valid, regardless of the diagnostic threshold chosen. The aim of this study was to evaluate reproducibility of the Nyvad criteria for clinical scoring of caries lesion activity, at different diagnostic thresholds, in adults with close inter-dental contacts in the permanent dentition. A voluntary sample of nineteen 18- to 
25-year-old students living in Minsk, Belarus, was selected. Prior to examinations, a full-day theoretical and practical training on the Nyvad criteria for caries lesion activity was provided (by B.N. and V.M.) for a group of three clinical examiners who had previously been trained with ICDAS criteria. Caries examinations were performed using intra-oral mirrors and sharp probes at two sessions with an interval of 1-2 days, using a dental equipment with non-standardized drying and light capacity. The inter- and intraexaminer reliability of 3 examiners (N.P., S.T., V.M.) were evaluated at three diagnostic thresholds: sound versus carious (any lesion); sound/inactive versus active; sound/intact versus cavitated. The inter-examiner agreement between 3 examiners was lowest when assessing sound versus carious: $89.4-90.8 \%$ (kappa $0.72-$ $0.77)$. For sound/inactive versus active, and for sound/intact versus cavitated it was $93.1-93.7 \%$ (kappa $0.48-0.57$ ), and $97.2-97.4 \%$ (kappa 0.84-0.86), respectively. The intra-examiner agreement of three examiners, at all thresholds, varied between 91.1 and $98 \%$ (kappa 0.49-0.89). The differences were statistically significant mostly when diagnosing active caries lesions, particularly when newly trained examiners were involved. In conclusion, the reproducibility of the Nyvad criteria, particularly for newly trained examiners, was moderate. More time for practical training of using the criteria, and the standardized examination conditions is recommended.

Supported by the ORCA Consultancy Service.

\section{1 \\ Near Infra-Red Imaging Technique for Early Dental Caries Detection: An in vitro Comparison with ICDAS Clinical Scoring System

\author{
S. Salsone ${ }^{\mathrm{a}, *}$, A. Taylor ${ }^{\mathrm{b}}$, J. Gomez ${ }^{\mathrm{b}}$, R. Ellwood ${ }^{\mathrm{b}}$, I. Pretty ${ }^{\mathrm{b}}$, \\ C. Zakian ${ }^{\text {b }}$ \\ silvia.salsone@unical.it \\ Manchester, UK
} \\ aUniversity of Calabria, Rende, Italy; ' University of Manchester,}

Near-infrared (NIR) reflectance imaging is a promising novel technology for caries detection at early stages of development. It has been demonstrated that NIR hyperspectral imaging offers numerous advantages as compared to visible light imaging. These include a deeper light penetration through the tooth revealing lesions not easily diagnosed and reduced absorption by stain, normally acting as a strong confounding factor. In addition, it is also known that radiography is not suitable for enamel lesion detection. The aim of this study is to compare NIR spectral imaging with ICDAS scoring system. We propose a novel method based on spectral imaging at wavelengths in the range from 1,000 to 1,700 $\mathrm{nm}$ taken for a total of 50 extracted teeth (premolars and molars) uniformly distributed across 5 ICDAS groups, from 0 to 4 . For each tooth, occlusal surface images at different wavelengths were combined to generate a quantitative lesion map. Such combina- tion consists of the image difference between two relevant wavelengths, giving information on presence of lesions (1,450 and $1,550 \mathrm{~nm}$ ), and normalised with respect to a reference wavelength $(1,050 \mathrm{~nm})$ to compensate for the natural brightness of the tooth. The maximum value of the resulting image was used as NIR caries score. A significant correlation (Spearman's coefficient $=0.59$, $\mathrm{p}<0.01$ ) between this NIR caries score and ICDAS code was found, with sensitivity of $90 \%$ and specificity $60 \%$ for lesions starting on the enamel and sensitivity $90 \%$ and specificity of $77.5 \%$ for lesions at the dentine according to ICDAS. Our study suggests that NIR spectral imaging is a novel method that can be used for the non-invasive detection and mapping of occlusal caries with a reliability comparable to ICDAS.

University of Manchester Intellectual Property, Ltd., UK; Ministero dell'Istruzione, dell’Università e della Ricerca, Italy.

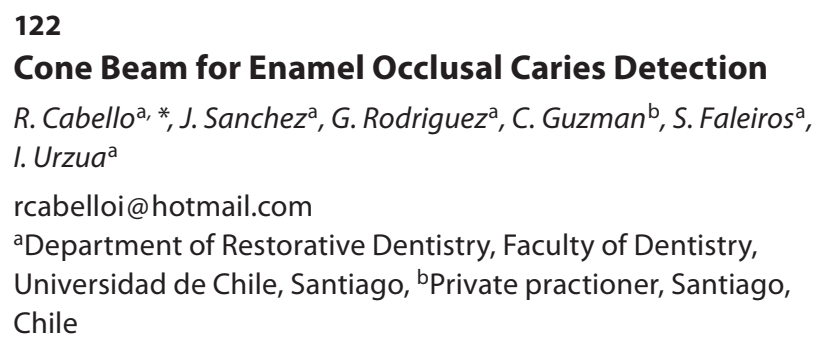

Detection of occlusal dental caries lesion had been an important issue to dentists. The aim of this study was to determine the sensitivity, specificity and ROC curve of digital high-resolution cone beam images for occlusal enamel caries detection. Non-restored, extracted permanent human premolar and molar teeth were mounted and imaged with a 3DX Siemens-Sirona, Galileo model. 125 occlusal surfaces were selected and assessed by 5 dentists using a five-step confidence scale on images obtained of cone beam for detection of enamel caries on occlusal surfaces $(1=$ caries definitely absent; 2 = caries probably absent; 3 = unsure if caries is absent or present; $4=$ caries probably present; $5=$ caries definitely present). Cone beam category 3 , the questionable category, was excluded from data analysis (observer $1=23.3 \%$; observer $2=5.6 \%$; observer $3=0 \%$; observer $4=7.2 \%$; observer $5=$ $12 \%)$. After evaluation, teeth were prepared histologically and assessed by an expert group for the presence of caries which served as the gold standard (any sign of demineralization was considered as caries present). One lesion was lost and not available for histological examination. Mean of sensitivity, specificity and ROC curve were calculated. A total of 87 enamel occlusal caries lesions were identified by histological observation. For occlusal caries lesion detection in enamel, sensitivity and specificity were 59.4 and $47.6 \%$ respectively. ROC area was 0.53 with a $95 \%$ of confidence interval with upper limit of 0.63 and a lower limit of 0.44 . The data suggests that cone beam has low sensitivity and low specificity for occlusal caries lesion detection in enamel.

Department of Restorative Dentistry funding. 


\section{Caries Research}

\section{Session 7 Microbiology}

\section{3 \\ Distribution of Streptococcus mutans Strains with Collagen-Binding Activities and Their Adhesion Properties to Endothelial Cells}

R. Nomura*, K. Nakano, S. Naka, M. Matsumoto, T. Ooshima

rnomura@dent.osaka-u.ac.jp; ooshima@dent.osaka-u.ac.jp Osaka University Graduate School of Dentistry, Osaka, Japan

Streptococcus mutans, a major pathogen of dental caries, is occasionally isolated from blood of patients with infective endocarditis (IE). S. mutans strains are classified into 4 serotypes, with approximately $70-80 \%$ of clinical isolates classified as serotype c, followed by e (approx. 20\%), while the distribution rates of serotypes $f$ and $\mathrm{k}$ are less than $5 \%$ each. As for virulence for IE, bacterial attachment to exposed collagen tissue is considered to be important. An approximately $120-\mathrm{kDa} \mathrm{Cnm}$ protein that binds to type I collagen has been identified in $10-20 \%$ of tested S. mutans clinical isolates, while there are several $S$. mutans strains without $\mathrm{Cnm}$ that have collagen-binding properties. We recently characterized a novel collagen-binding protein named $\mathrm{Cbm}$ that has an approximately $80 \%$ homology with the collagen-binding domain of Cnm. In the present study, we analyzed $460 \mathrm{~S}$. mutans strains isolated from the oral cavities of 300 Japanese, 110 Finish, and 50 Thai subjects. PCR analyses revealed that 52 (11.3\%) and $11(2.4 \%)$ of the strains were cnm-positive and cbm-positive, respectively. In addition, strains with the $\mathrm{Cnm}$ proteins were predominately serotype $\mathrm{f}$, while those with $\mathrm{Cbm}$ were predominately serotype $\mathrm{k}$. Following insertional inactivation of the $\mathrm{cnm}$ or cbm gene, which encode $\mathrm{Cnm}$ and $\mathrm{Cbm}$, respectively, the isogenic mutant strains showed a lack of collagen-binding activities. Finally, the adhesion properties of $S$. mutans to human umbilical vein endothelial cells were evaluated. Strains with $\mathrm{cnm}$ or $\mathrm{cbm}$ showed significantly higher adhesion rates than $\mathrm{cnm}$-inactivated or cbm-inactivated isogenic mutant strains. Together, our results indicate that $\mathrm{Cnm}$ and $\mathrm{Cbm}$ proteins are highly prevalent in strains with minor serotypes, such as $\mathrm{f}$ and $\mathrm{k}$, which may be highly virulent for the development of IE.
This study was supported by the Grants-in-Aid for Young Scientists (B) 21792067 from the Ministry of Education, Culture, Sports, Science and Technology of Japan.

\section{4 \\ Caries Progression from the Viewpoint of Bacteriocinogenicity of Streptococcus mutans \\ S. Kneista ${ }^{\mathrm{a}}{ }^{*}$, M. Bratanow ${ }^{\mathrm{a}}$, B. Willershausen ${ }^{\mathrm{b}}$, H. Küpper ${ }^{\mathrm{a}}$, A. Callaway ${ }^{b}$ \\ Susanne.Kneist@med.uni-jena.de \\ aDental School, Biological Laboratory, Clinic for Prosthetic \\ Dentistry and Dental Materials, University of Jena, and \\ bUniversity of Mainz Dental School, Germany}

The extent of bacteriocinogenicity in Streptococcus mutans was studied in 135 strains from hard carious dentine of 8 deciduous molars with caries profunda from 6 eight-year-old children. After caries removal (Carisolv ${ }^{\mathrm{TM}}$ ), samples were taken from the hard cavity floor (U1), the cavities received temporary fillings, and from the hard carious dentine after 8 weeks of clinical observation (U2). 69 strains were isolated at U1, 66 at U2; all strains were serotype c. Bacteriocinogenicity was tested for strains originating from the same tooth, using bacteriocin fingerprinting. First, strains from U1 served as test strains (TS), from U2 as indicator strains (IS), then vice versa. TS were grown anaerobically, $24 \mathrm{~h}$, on a base agar as macrocolonies. The TS macrocolonies from $\mathrm{U} 1(8.4 \pm 0.5 \mathrm{~mm})$ and $\mathrm{U} 2(7.7 \pm 0.5 \mathrm{~mm})$ had the same mean size $(\mathrm{p}>0.05)$. The macrocolonies were overlaid with an agar containing the corresponding IS, and incubated anaerobically, $24 \mathrm{~h}$. Inhibition zones (IZ) in lawns of IS sized $9.3 \mathrm{~mm}$ (U1) or $9.0 \mathrm{~mm}$ (U2) were considered as TS bacteriocinogenicity. Means and standard deviations of IZ were calculated, differences were analyzed with the Wilcoxon test $(\alpha=0.05)$ and the Pareto distribution. Per cavity results from $70 \pm 35$ pairs of strains (U1, U2) were avail- 
able. 57 TS from U1 and 48 TS from U2 were bacteriocinogenic to all IS from the same cavity. The size of the IZ was 7.5-19 mm (U1) or $7.7-20 \mathrm{~mm}$ (U2). The IZ caused by U1 strains were significantly smaller than those by U2 strains. According to the Pareto distribution, $30 \%$ each of the strains from U1 and U2 were more strongly bacteriocinogenic. When using the strain from U1, producing the most bacteriocin as reference, $41 \%$ of the U2 strains were more strongly bacteriocinogenic. During caries progression, bacteriocinogenicity and virulence in $S$. mutans can increase.

University of Jena and Mainz.

\section{5 \\ Effect of Fluoridated Milk on Streptococcus mutans Biofilm Properties and Enamel and Dentin Demineralization}

\author{
R.A. Giacaman ${ }^{\mathrm{a}, *}$, M.J. Muñoz ${ }^{\mathrm{a}}$, R.A. Ccahuana ${ }^{\mathrm{b}}$, C. Muñoz $^{\mathrm{a}}$, \\ J.A. Cury ${ }^{\mathrm{b}}$ \\ giacaman@utalca.cl \\ ${ }^{a}$ Cariology Unit, Department of Oral Rehabilitation, University

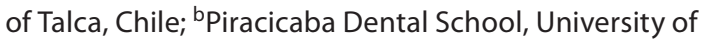 \\ Campinas, Piracicaba, Brazil
}

Evidence shows that milk fluoridation may be effective to control enamel caries but its effect on dentine is unclear. Likewise, its effect on the oral biofilm has not been explored. Streptococcus mutans (UA159) biofilms formed on enamel and root dentin slabs under $10 \%$ sucrose eight times/day were exposed twice a day to one of the following treatments: (1) $0.9 \% \mathrm{NaCl}$ (negative control), (2) bovine milk, (3) fluoridated bovine milk (5 ppm NaF) and (4) $\mathrm{NaF} 0.05 \%$ (anticaries positive control). Medium $\mathrm{pH}$ was monitored as an indicator of biofilm acidogenicity. After 4 days for dentine and 5 for enamel, biofilms were collected for analyses: viable microorganisms, biomass, protein content and extra- and intracellular polysaccharides. On the tooth slabs, demineralization was assessed by surface hardness loss (\%SHL). The experiment was conducted in triplicate and repeated 3 times. Results were compared by ANOVA followed by Tukey test. Biofilm acidogenicity did not differ among the treatment groups. Analysis of the biofilms failed to show differences among the groups, neither for enamel nor for root dentin biofilms $(p>0.05)$. Demineralization in enamel was similar between biofilms exposed to fluoridated milk and $\mathrm{NaF}$, but lower than those treated with non-fluoridated milk and with the negative control $(\mathrm{p}<0.05)$. Despite a marked trend for lower demineralization, $\% \mathrm{SHL}$ of root dentin slabs treated with fluoridated milk resulted statistically similar to nonfluoridated milk-exposed biofilms ( $\mathrm{p}>0.05$ ), but lower than slabs treated with $0.9 \% \mathrm{NaCl}$. These findings strongly suggest that $5.0 \mathrm{ppm}$ fluoridated milk is effective at reducing enamel caries but may not significantly influence dentine demineralization.

This research has been partly funded by Grant FAPESP2009/07019-3 to J.A.C. and Grant \#I001068 of the University of Talca to R.A.G.

\section{6 \\ Salivary Levels of Bifidobacteria Are Significantly Correlated with Caries in Children}

\author{
R. Kaur, S.C. Gilbert, E. Sheehy, D. Beighton* \\ david.beighton@kcl.ac.uk \\ Infection Research Group, KCL Dental Institute, London, UK
}

Bifidobacteria are predominant aciduric and acidogenic commensal bacteria present in the oral biofilm associated with dental caries and salivary levels in adults correlate with caries experience. Here we have investigated the association between the salivary levels of bifidobacteria and caries experience in children. Caries active $(\mathrm{n}=38$; mean affected surfaces $13.1 \pm 7.1)$ and caries-free $(\mathrm{n}=20)$ children were included. Mixed saliva was collected using the tongueloop method with $10 \mu \mathrm{l}$ of saliva being dispersed into $1 \mathrm{ml}$ of broth. Duplicate $100 \mu \mathrm{l}$ samples were spread onto media for the enumeration of bifidobacteria, mutans streptococci, lactobacilli and yeasts; the detection limit was $500 \mathrm{cfu} / \mathrm{ml}$. Caries was determined by visual examination in conjunction with radiographs and caries experience decayed/missing/filled surfaces and teeth was calculated. Other variables including a plaque index, oral hygiene status, frequency of sugar intake and fluoride usage were also recorded. Nonparametric univariate analyses were performed and discriminate analysis was used to determine which variables [excluding caries experience] identified the two groups. The mean ( \pm SD) salivary levels $\left[\right.$ as $\left.\log _{10}(\mathrm{cfu} / \mathrm{ml})\right]$ of bifidobacteria, mutans streptococci and lactobacilli and the PI in the caries group were $4.41 \pm 0.92,4.41 \pm$ $0.84,3.53 \pm 1.09$ and $0.83 \pm 0.89$, respectively compared to $2.43 \pm$ $0.80,2.20 \pm 0.63,2.21 \pm 0.44$ and $0.09 \pm 0.23$ in the caries-free group (all $\mathrm{p}<0.001$ ). For the whole population caries experience correlated $(\mathrm{p}<0.001)$ with salivary bifidobacteria $(0.732)$, mutans streptococci (0.696), lactobacilli (0.741) and with PI (0.531). When all variables were entered into the discriminate analysis only the salivary levels of bifidobacteria, mutans streptococci and oral hygiene score were significant variables correctly classifying $90 \%$ of the subjects in both groups. Salivary concentrations of bifidobacteria were significantly correlated with caries experience in children.

The project was partially funded by the Department of Health via the National Institute for Health Research (NIHR) Comprehensive Biomedical Research Centre Award to Guy's and St Thomas' NHS Foundation Trust in partnership with King's College London and King's College Hospital NHS Foundation Trust.

\section{7 \\ Colonization of the Oral Cavity by Probiotic Bacteria I. Ravn ${ }^{\mathrm{a}, *}$, I. Dige , R.L. Meyer, C, E. Brockmann ${ }^{\mathrm{d}}$, B. Nyvad ${ }^{\mathrm{a}}$ \\ nyvad@odont.au.dk \\ aSchool of Dentistry, Aarhus Faculty of Health Sciences, Aarhus University, ${ }^{b}$ Department of Biological Sciences, Aarhus Faculty of Science and Technology, Aarhus University, Interdisciplinary Nanoscience Center, Aarhus Faculty of Science and Technology, Aarhus University, and ${ }^{\mathrm{d} I n n o v a t i o n, ~ C h r . ~ H a n s e n, ~ D e n m a r k ~}$}

Probiotic dairy products contain bacteria that are intended to preserve a healthy gut microflora and/or stimulate the immune system. There is hardly any information about the colonization of 
probiotic bacteria in the oral cavity of subjects who consume probiotic dairy products on a regular basis. Clinical studies have suggested that probiotic bacteria have a beneficial effect on caries. However, their acidogenic and aciduric properties may also be harmful if they colonize the tooth surfaces. The aim of this study was firstly to design and/or optimize oligonucleotide probes for in situ detection of the probiotic bacteria Bifidobacterium animalis subsp. lactis $\mathrm{BB}-12^{\circledR}$, Lactobacillus acidophilus LA $-5^{\circledR}$, and L. paracasei subsp. paracase $\mathrm{F} 19^{\circledR}$ present in the probiotic fermented milk product Cultura Dofilus ${ }^{\circledR}$ naturell. Secondly, we investigated if the probiotic bacteria were present in saliva and on oral mucosal surfaces of two caries-inactive individuals, and if they colonized dental surfaces in situ up to three days under the influence of eight daily exposures to Cultura Dofilus ${ }^{\circledR}$ naturell. We took a culture-independent approach in which the probiotic bacteria were identified by fluorescence in situ hybridization (FISH) and confocal laser scanning microscopy. While probiotic bacteria were present sporadically in the oral cavity on mucosal surfaces and in saliva after three days of frequent use of the probiotic milk, they were not detected on dental surfaces. Probiotic bacteria may thus contribute to the general oral health, but our results indicate that their potential detrimental role in acid production on tooth surfaces did not appear to be relevant. Future intervention studies using molecular identification methods in situ may further improve our understanding of the mode of action behind the effect of probiotic products in the oral cavity.

We gratefully acknowledge the financial support from the Danish Dental Association (FORSKU) for funding dental student Ina Ravn while working with this project. We thank the Lundbeck Foundation, Denmark, for funding Rikke Meyer. Additional financial support was received from Colgate Palmolive A/S, Copenhagen, Denmark. Arla Foods Amba, Stockholm, Sweden, donated fermented milk products for the in situ studies.

\section{8 \\ Inhibition of Streptococcus mutans by Dental Flosses Impregnated with Different Antibacterial Substances in vitro}

U. Schiffner*, P. Aumüller

schiffner@uke.uni-hamburg.de

University of Hamburg Dental School, Germany

Several commercially available dental flosses are supplied with different antibacterial substances. However, there is little knowledge about the bacteria inhibiting effect of these supplies. The aim of this in vitro study was to determine and to compare the growth inhibiting effect of these flosses on Streptococcus mutans. 20 different dental floss brands from the European and the American market were included. Two served as unimpregnated controls, while the others contained amine fluoride (AmF), calcium peroxide (Cal), cetylpyridinium chloride (CPC), chlorhexidine digluconate (CHX), cranberry-extract (Cra), different essential oils (OIL), an enzyme complex (neoE), green tea-extract (GTE), mince oil (spe), silver nitrate $(\mathrm{AgN})$, sodium fluoride $(\mathrm{NaF})$, tea-tree oil (TTO) and xylitol (Xyl). The growth inhibiting effect was tested using an agar diffusion test. A cariogenic human
S. mutans strain was inoculated on selective Mitis-Salivarius agar. Ten replicate $1.5 \mathrm{~cm}$ long pieces of each floss were applied to the agar. After $40 \mathrm{~h}$ anaerobic incubation (N2 95\%, $\mathrm{CO}_{2} 5 \%$ ) at $37^{\circ} \mathrm{C}$ the size of an inhibition zone was measured perpendicular to the length of the floss. No inhibition was found with the controls or in the flosses with Cal, spe, GTE, TTO, neoE and AgN. Minor inhibition areas up to $0.35 \pm 0.07 \mathrm{~mm}$ were found next to the flosses with $\mathrm{Cra}, \mathrm{Xyl}$, and $\mathrm{NaF}$, while greater areas $(1.04 \pm 0.12$ to $3.84 \pm 0.48 \mathrm{~mm}$ ) could be determined next to the flosses with AmF, CHX, or CPC. The inhibition observed with flosses containing CPC or CHX (3.42-3.84 mm) were statistically different $(\mathrm{p}<0.05)$ from all other flosses (ANOVA, Tukey's post-hoc test). The study shows that some dental flosses which are impregnated with antibacterial substances might have the potential to inhibit the growth of $S$. mutans.

\section{9 \\ Drinking Oolong Tea Inhibits Dental Plaque Accumulation in Humans \\ M. Matsumoto-Nakano ${ }^{\mathrm{a},}$ *, K. Fujita ${ }^{\mathrm{a}}$, S. Inagaki ${ }^{\mathrm{a}}$, K. Nagayama ${ }^{\mathrm{a}}$, Y. Takashima ${ }^{a}$, M. Kontani $^{\mathrm{b}}$, Y. Kiso $^{\mathrm{b}}$, T. Ooshima $^{\mathrm{a}}$ \\ michiyo@dent.osaka-u.ac.jp \\ ${ }^{a}$ Department of Pediatric Dentistry, Osaka University Graduate School of Dentistry, and ' Institute for Health Care Science, \\ Suntory Wellness Ltd., Japan}

Streptococcus mutans, implicated as a primary causative agent of dental caries in humans, synthesizes water-insoluble glucan from sucrose by the action of glucosyltransferases (GTFs). We previously reported that oolong tea polyphenols inhibited the function of GTFs in vitro, while oolong tea extract added to drinking water reduced plaque accumulation and inhibited the development of experimental dental caries in rats. The aim of the present study was to analyze the inhibitory effects of drinking oolong tea on dental plaque in humans. We enrolled 31 volunteers, who participated according to a study protocols approved by the Ethics Committee of Osaka University Graduate School of Dentistry. Prior to the experiment, dental plaque on the tooth surfaces of the subjects was professionally removed, after which no other oral hygiene procedures were performed, including by the subjects. They were randomly divided into 2 groups. For 4 day, the subjects were asked to refrain from drinking tea, coffee, and alcohol, during which one group drank only oolong tea and the other drank only water at every meal (at least $200 \mathrm{ml}$ each time) and before sleeping (at least $100 \mathrm{ml}$ ). On the 4th day, the degree of plaque accumulation was evaluated after staining with erythrocin. The next week, the drinking contents were switched between the groups and the same protocol was followed. The plaque indexes of subjects who drank oolong tea were significantly lower $(\mathrm{p}<0.01)$. We concluded that drinking oolong tea is a useful means of inhibiting dental plaque formation.

This work was supported in part by the Fund for Suntory Ltd. Osaka, Japan. 


\section{0 \\ In Depth Characterization of the Oral Microbiome}

J.M. ten Cate*, M.J. Buijs, W. Crielaard, E. Zaura

j.t.cate@acta.nl

Academic Center for Dentistry Amsterdam (ACTA),

The Netherlands

Traditionally research to develop antimicrobials focused on a few bacterial species which were held responsible for caries and periodontal infections. Recent data have shown that the oral microbiota is considerably more complex, with about hundred bacterial taxa being represented in each individual oral sample, and large site to site variations within the oral cavity. The aim of this study was to determine bacterial composition/diversity and intra/ intersubject variations in three samples taken from buccal dental surfaces ('plaque'), saliva and tongue, respectively, using sequencing method to analyze the samples at $0.01 \%$ sensitivity. Nine healthy subjects donated 4 sets of samples over a period of 10 weeks. The study was approved by the university review board. Samples were analyzed by 454 pyrosequencing and assessed by principal component analysis (PCA) and ecological indices: Dominance D and Shannon H Diversity index. The combined samples resulted in 950,000 reads with 374,500 unique sequences. Of all reads, 93\% could be classified at least to phylum level (12 phyla), $89 \%$ - to class, $86 \%$ - order, $72 \%$ - family and $55 \%$ - to genus level (87 genera). Clustering of sequences at $6 \%$ similarity resulted in 7,256 operational taxonomic units (OTUs). On average, each sample contained 668 (SD 129) OTUs, with no statistical differences among the sample types $(\mathrm{p}=0.055$, ANOVA). Plaque and tongue samples differed significantly in their Dominance and Diversity indices, with plaque samples being more dominated by abundant taxa ( $\mathrm{p}=0.021$, ANOVA, Games-Howell post-hoc test), and tongue samples showing higher diversity $(\mathrm{p}=0.019)$. PCA discriminated between the samples by the sampling site, with plaque being distinctly different from saliva and tongue samples. PCA clustered samples at subject level indicating that differences between subjects were larger than within the subjects.

\section{1 \\ Evaluation of Plate Counts Assays for the Enumeration of Dual-Species Bacterial Cultures \\ X.L. Li ${ }^{\mathrm{a}}$ b, * , J.Q. Ling $^{\mathrm{b}}$, W. Crielaard ${ }^{\mathrm{a}}$, D.M. Deng ${ }^{\mathrm{a}}$ \\ lingjq@mail.sysu.edu.cn \\ aDepartment of Experimental Preventive Dentistry, Academic \\ Centre for Dentistry Amsterdam, University of Amsterdam and Free University Amsterdam, The Netherlands; ${ }^{b}$ Research Institute of Stomatology, Guanghua School of Stomatology, Sun Yat-Sen University, Guang-zhou, China}

Though modern molecular techniques have been increasingly applied for bacterial enumeration in clinical or laboratory samples, conventional plate counts are still being used as the gold standard in many studies. Due to the lack of selective plates, different species growing on one agar plate were often identified by morphology differences and counted. The aim of this study is to eval- uate the accuracy of plate counts when two different species are plated on one plate. Three clinical isolates of the cario-pathogen Streptococcus mutans (UA159, C180-2, C67-1) and two clinical strains of the endodontic pathogen Enterococcus faecalis (ER5/1, OG1X) were grown overnight. Cultures of one $S$. mutans and one $E$. faecalis strain were mixed at the different ratios and plated on Brain-Heart-Infusion agar plates after serial dilutions. The identification of each strain is based on its colony morphology and the CFUs of each strain were then counted (aCFU). The overnight culture of each single strain was also plated and used to calculate the expected CFU counts (eCFU) in the mixture. The eCFU values were compared to aCFU values by a paired t test. It was found that the aCFU counts of both $E$. faecalis strains were consistently higher than expected, irrespective of the strains of $S$. mutans in the mixture and the mixing ratio. While the aCFU counts of three $S$. mutans strains generally agreed with the expected value. When the E. faecalis cells were 500 or 100 times higher than S. mutans cells in the mixture, significant differences between aCFU and eCFU could be seen for strain C180-2 and UA159. In conclusion, plate count assays in this study resulted in the overestimation of one of the bacterial species in mixed species samples.

Supported by a State Sponsored Graduate Scholarship Program for Building High-Level Universities, China.

\section{2 \\ An Improved Artificial Saliva for Screening of Cariogenic Effect of Carbohydrates}

\section{S.D. Forssten*, M. Björklund, A.C. Ouwehand}

sofia.forssten@danisco.com

Danisco BioActives, Health and Nutrition, Kantvik, Finland

An artificial saliva with nutrient levels resembling those in natural saliva was developed as a substitute for natural human saliva to study the influence of different carbon sources on the growth of Streptococcus mutans. Saliva is a complex fluid that possesses many important functions regarding oral health. Brain Heart Infusion (BHI) has been commonly used for e.g. biofilm studies, but being a nutrient rich medium it does not mimic the physicochemical properties of saliva. While natural saliva would be the material of choice, it varies in composition between and within individuals and it may be difficult to obtain in sufficient quantities. The growth of two wild type $S$. mutans strains and S. mutans ATCC 15175 were assessed anaerobically in artificial saliva, stimulated human saliva and BHI, to which glucose, sucrose and xylitol were added as carbon sources. The growth was monitored by automatic measurement of absorbance at $600 \mathrm{~nm}$. The growth of the $S$. mutans strains was significantly higher in BHI ( $<$ 0.0005), in comparison to the stimulated human saliva. For most tested carbon sources, growth of $S$. mutans was similar in artificial saliva compared to stimulated saliva. Since the growth of the $S$. mutans strains in the artificial saliva resembled the growth in natural human saliva, it can be concluded that the improved artificial saliva is suitable for in vitro tests on the effect of carbohydrates on the growth of $S$. mutans.

The project has been fully financed by Danisco. 


\section{3 \\ Effect of Gallium on Streptococcus mutans NCTC10449 and Dental Mineralisation}

S.P. Valappil*, G.J. Owens, S.M. Higham

S.Valappil@liv.ac.uk

School of Dentistry, University of Liverpool, UK

Gallium doped phosphate-based glasses (PBGs) are biodegradable, biocompatible materials which may significantly impact on Streptococcus mutans, a caries-associated bacterium, and dental mineralisation. Gallium doped and control gallium free PBG rods $(5 \times 2 \mathrm{~mm})$ were produced using a conventional melt quenching method, at $1,100^{\circ} \mathrm{C}$ for $1 \mathrm{~h}$. Degradation studies $(0$ $48 \mathrm{~h}$ ) were carried out in triplicate using a weight loss method in $\mathrm{dH}_{2} \mathrm{O}$ at $37 \pm 1^{\circ} \mathrm{C}$ and starting $\mathrm{pH}$ of $7 \pm 0.1$. For antibacterial assay, the glass discs were placed on fastidious anaerobic agar plates previously inoculated with standardised cultures of $S$. $m u$ tans NCTC10449 and incubated for $24 \mathrm{~h}$ aerobically at $37^{\circ} \mathrm{C}$. The diameters of zones of inhibition that developed around the glass samples were measured. The effect of gallium exposure $(0-128$ $\mathrm{ppm}$ ) on dental mineral was investigated in a static liquid medium $\left(\mathrm{dH}_{2} \mathrm{O}, 0-128\right.$ ppm $\mathrm{Ga}$ from $\left.\mathrm{Ga}\left(\mathrm{NO}_{3}\right)_{3}\right)$. Following $15-240 \mathrm{~min}$ exposure, minerals were extracted and digested in $35 \% \mathrm{HNO}_{3}$. Calcium, gallium and phosphate concentrations remaining in solution and in $\mathrm{HNO}_{3}$ digests were measured by inductively-coupled optical-emission spectrometry (ICP-OES). The degradation rate of the gallium doped PBG was $17.50 \mu \mathrm{g} \cdot \mathrm{mm}^{-2} \cdot \mathrm{h}^{-1}$ compared with controls, $41.60 \mu \mathrm{g} \cdot \mathrm{mm}^{-2} \cdot \mathrm{h}^{-1}$. The results showed that ion release rates are correlated to the weight losses of the glasses. Zones of inhibition were found to be $10.5 \pm 0.5 \mathrm{~mm}$ for the gallium doped PBG glasses compared with controls. ICP-OES analyses confirmed transient gallium adsorption into the mineral, most likely through the displacement of calcium ( $\geq 15$ min exposure). Gallium moiety, i.e. $\mathrm{Ga}^{3+}$ ions that has the potential to disrupt $\mathrm{Fe}^{3+}$ metabolism of $S$. mutans, of PBG glasses found to inhibit $S$. mutans growth and it displayed no adverse effect on dental mineral.

Induction Award, University of Liverpool.

\section{4 \\ Time-Kill Determination of Croton doctoris Hydroalcoholic Extract against Cariogenic Bacteria}

F.L.Brighenti, ${ }^{a}{ }^{*}$, A.C.B. Delbem ${ }^{b}$, M.J. Salvador ${ }^{c}$, A.C.B. Delbem ${ }^{\mathrm{a}}$, L.S.F. Freitas ${ }^{\text {a }}$ M.A.C. Oliveira ${ }^{\text {a }}$, C.Y. Koga-Ito ${ }^{\text {a }}$

ferbrighenti@gmail.com

aUNESP - Universidade Estadual Paulista, São José dos Campos,

bUNESP - Universidade Estadual Paulista, Araçatuba, and

'UNICAMP, Campinas, Brazil

Croton doctoris hydroalcoholic extract had its anticariogenic activity recently found (Brighenti et al: Caries Res 2010;44:207). The aim of this study was to evaluate the killing kinetics of this extract against cariogenic bacteria. Dried leaves were macerated with $70 \%$ ethanol $(1: 20 \mathrm{w} / \mathrm{v})$ for $72 \mathrm{~h}$ at room temperature. Ethanol was evaporated under reduced pressure. The reference strains used were: Actinomyces naeslundii ATCC 19039, Lactobacillus acidophilus ATCC 4356, Streptococcus mutans ATCC 35688, and S. sobrinus ATCC 33478 . Fresh inocula were prepared in BHI broth from a $1,000,000 \mathrm{CFU} / \mathrm{ml}$ bacterial suspension. Then the suspensions were incubated $\left(37^{\circ} \mathrm{C}, 5 \%\right.$ carbon dioxide) until the microorganisms were half way into their exponential growth phase. The extract was added at concentrations of 1,2 or 4 times the MIC and the suspensions were treated for 5, 15, 30, 60 and 120 min. Negative control (BHI broth) and positive control $(0.12 \%$ chlorhexidine digluconate) were included. The suspensions were diluted and plated on BHI agar. After incubation for $48 \mathrm{~h}$ at $5 \%$ $\mathrm{CO}_{2}$, the number of colony-forming units $(\mathrm{CFU} / \mathrm{ml})$ was obtained. S. mutans showed a reduction of $53 \%$ on viable cells after $15 \mathrm{~min}$ treatment. L. acidophilus showed a reduction of $52 \%$ on viable cells after $30 \mathrm{~min}$. S. sobrinus was the most susceptible microorganism and showed no viable cells after 120 min treatment. The most resistant microorganism was A. naeslundii (reduction of only $29 \%$ after $120 \mathrm{~min}$ ). A dose-response relationship was not observed for the extract. In conclusion, $C$. doctoris extract is effective against cariogenic bacteria and is a promising anticariogenic substance.

FAPESP (08/04114-2 and 2008/53299-5).

\section{5 \\ Effect of Croton doctoris Extract on the Adhesion of Bacteria with Cariogenic Relevance}

F.L. Brighenti ${ }^{\mathrm{a}}$, C.M. Girondi ${ }^{\mathrm{a}}$, A.C.B. Delbem ${ }^{\mathrm{b}}$, M.J. Salvador , A.C.B. Delbem ${ }^{\mathrm{a}}$, C.Y. Koga-Ito ${ }^{\mathrm{a}, *}$

cristiane@fosjc.unesp.br

aUNESP - Universidade Estadual Paulista, São José dos Campos,

bUNESP - Universidade Estadual Paulista, Araçatuba, and

'UNICAMP, Campinas, Brazil

Croton doctoris is a native plant from Brazilian Pantanal. This study aimed to evaluate the effect of its hydroalcoholic extract on the adhesion of bacteria with cariogenic relevance. Dried leaves were macerated with $70 \%$ ethanol $(1: 20 \mathrm{w} / \mathrm{v})$ for $72 \mathrm{~h}$ at room temperature. Ethanol was evaporated and the extract was re-suspended in BHI broth just before use. The anti-adhesion activity was distinguished from growth inhibition by using sub-inhibitory extract concentrations (50 and $25 \%$ of the minimal inhibitory concentration [MIC]). Standardized suspensions $\left(10^{6} \mathrm{cfu} / \mathrm{ml}\right)$ of Streptococcus sanguinis ATCC 10556, S. mutans ATCC 35688, Lactobacillus acidophilus 4356, S. sobrinus ATCC 33478 and S. gordonii ATCC 10558 were prepared in $\mathrm{NaCl} 0.9 \%$ (w/v) solution. Standardized glass specimens ( $\varnothing 1.5 \mathrm{~mm}, 30 \mathrm{~mm}$ length; $\mathrm{n}=$ 10 /group) were exposed to bacterial suspensions ( $1 \%$ final concentration) in Brian Heart Infusion and the extract (50 and 25\% subMIC). Controls (BHI plus $0.12 \%$ chlorhexidine digluconate and $\mathrm{BHI}$ with no additions) were also prepared. After incubation for $24 \mathrm{~h}$ at $37^{\circ} \mathrm{C}$, adhered cells were dispersed and suspensions were plated onto $\mathrm{BHI}$ agar, incubated for $48 \mathrm{~h}$ at $37^{\circ} \mathrm{C}$ and $5 \% \mathrm{CO}_{2}$ and the number of cfu was obtained. Data were analyzed by ANOVA and Tukey's test (5\%). C. doctoris extract at 50\% MIC significantly reduced adhesion of all tested species. The extract at $25 \%$ MIC was also able to reduce adhesion of $S$. sanguinis ( $\mathrm{p}<0.0001)$, S. sobrinus $(\mathrm{p}<0.0001)$ and S. gordonii $(\mathrm{p}=0.003)$. C. doctoris at 
50\% MIC showed similar counts when compared to chlorhexidine digluconate for all the tested species. This suggests that C. doctoris hydroalcoholic extract has a promising anti-adhesion activity on cariogenic bacteria.

FAPESP (08/04114-2 and 2008/53299-5).

\section{6 \\ Importance of DNA Release in the Initial Phases of Biofilm Formation by Streptococcus mutans}

R.N.Stipp*, J.F. Höfling, R.O. Mattos-Graner

stippRN@gmail.com

Department of Oral Diagnosis, Piracicaba Dental School,

University of Campinas (UNICAMP), São Paulo, Brazil

Extracellular DNA (eDNA) is recognized as an important constituent of the biofilm matrix in several bacterial species. Streptococcus mutans, a dental caries pathogen, expresses glucosyltransferase genes $(g t f B / C)$ and $g b p B$, important for the synthesis of and interaction with glucan, a major component of biofilm matrix. The two-component systems CovR and VicRK up- and downregulate this process, respectively. The aim was to investigate the influence of eDNA in biofilm growth and to explore the role of VicRK and CovR for this process. Knock-out mutant strains covR- (UAcov) and vicK- (UAvic) were constructed in strain UA159 (WT). Biofilms were formed in chemically defined medium $(0.1 \%$ sucrose $w / v)$, supplemented or not with deoxyribonuclease I (DNAseI, $50 \mu \mathrm{g} / \mathrm{ml}$ ), which can cleavage single or double stranded DNA backbone. Heat-inactivated DNAseI was used as control. After $8 \mathrm{~h}$ growing, biofilms were photographed and growth measured. Extracellular matrix was alkali solubilized, neutralized, and cells separated by centrifugation. Supernatant containing eDNA was quantified by qPCR using primers for 16SRNA. Untreated biofilms formed by UAcov and UAvic were structurally distinct from that formed by parent. In DNAseI treated biofilms, qPCR confirmed eDNA was reduced up to 30 -fold. When compared to respective control without DNAseI, WT and UAvic strains formed less biofilm mass (2.3- and 6.5-fold, respectively) $(\mathrm{p}<0.01)$ and significant alterations in biofilm structure/ aspect. UAcov biofilm, despite structurally altered by DNAseI, didn't show biomass decrease. Late biofilms (18 h) formed with DNAseI did not show differences for all strains. eDNA may play an important role in the architecture and establishment of extracellular matrix formed by $S$. mutans in low sucrose conditions. Other components, regulated by CovR/VicKR, may interact with eDNA and contribute to biofilm shape.

CAPES PNPD 301/2010; FAPESP 09/54182-7.

\section{7 \\ Caries-Associated Risk Factors: The Relationship between the Host Immune Response and Oral Biofilm Development}

\author{
J. Malcolm ${ }^{\mathrm{a}}$, G. Ramage ${ }^{\mathrm{a}}$, L. Macpherson ${ }^{\mathrm{b}}$, D.I. Conway ${ }^{\mathrm{b}}$, Y. Blair $^{\mathrm{b}}$, \\ A. Sheriff ${ }^{b}$, S. Culshaw ${ }^{a, *}$ \\ shauna.culshaw@glasgow.ac.uk \\ anfection and Immunity, Glasgow Dental School, and \\ ${ }^{b}$ Dental Public Health Unit, Glasgow Dental School, \\ University of Glasgow, Glasgow, UK
}

Dental caries is a chronic infectious disease that remains highly prevalent worldwide. Dental caries is associated with specific pathogenic microorganisms, namely mutans streptococci, which colonize the teeth from around 18 months of age. Dental caries is a complex, multi-factorial disease, influenced by behavioral, dietary and socioeconomic factors. However, the disease results from bacterial infection and as such caries susceptibility can be affected by the host immune response. We therefore sought to investigate microbiological and immunological characteristics of dental plaque and saliva collected from children at the early stages of mutans colonization. Sixty-seven children were recruited with a mean age of $17.6 \pm 6.4$ months. Microbiological culture techniques and QPCR were used to quantify mutans streptococci. Saliva was investigated for levels of antimicrobial peptides (AMPs) and salivary IgA (SIgA) antibodies specific for oral streptococci by ELISA. Additionally, we sought to investigate if social inequalities relate to microbial colonization and immune responses. Deprivation may influence physiological stress, which may impact the host response. Salivary cortisol was measured as a surrogate measure of stress. Demographic data was collected from parents by questionnaire. Results: S. mutans isolated from 15 of 62 patients, with mean CFU/ml of $8.7 \times$ $10^{4} \pm 4.2 \times 10^{5}$. Cortisol, AMPs and SIgA antibodies were all detectable in saliva and each demonstrated extensive variation between individuals. Salivary $S$. mutans $\mathrm{CFU} / \mathrm{ml}$ increased with increased cortisol, however, this did not reach statistical significance. The levels of AMPs were significantly associated with increased bacterial load ( $\mathrm{p}<0.05)$ and LL37 and calprotectin were significantly associated with increased $S$. mutans in plaque $(\mathrm{p}<0.05)$. Further analysis will seek to identify cumulative relationships between immune responses, stress responses and oral biofilm development.

This work was funded by the Scottish Government Health Department and Tenovus Scotland. 


\section{8 \\ Red Fluorescence of Dental Plaque from Dental Students}

C.M.C. Volgenant ${ }^{\mathrm{a}, *}$, J.J. de Soet ${ }^{\mathrm{a}}$, E. de Josselin de Jong ${ }^{\mathrm{a}, \mathrm{b}}$, J.M. ten Cate ${ }^{\mathrm{a}}$, M.H. van der Veen ${ }^{\mathrm{a}}$

c.volgenant@acta.nl

${ }^{a}$ Academic Centre for Dentistry Amsterdam (ACTA), University

of Amsterdam and VU University Amsterdam, Department

of Preventive Dentistry, and ${ }^{b}$ Inspektor Research Systems BV,

Amsterdam, The Netherlands

Dental plaque fluoresces bright red after excitation at $405 \mathrm{~nm}$. It is assumed that matured plaque is responsible for this fluorescence. The aim of this clinical study is to investigate the relation between the amount of red fluorescent plaque on the buccal surfaces of the teeth and the percentage of Gram positive bacteria in the dental plaque by using a Gram stain. From the 126 first year dental students from the Academic Centre for Dentistry Amsterdam, 119 students $(94.4 \%)$ consented to participate in this study, mean age 20.2 years \pm 2.5 SD (range $17-30$ years, 39.5\% male, $60.5 \%$ female). Series of white light and fluorescence photographs were taken of each student with the QLF-D SLR camera (Inspektor Research Systems, Amsterdam) from the front and the right buccal side. Plaque samples of unknown age were taken from the second upper molar on the right side (tooth 17), Gram stained and judged microscopically for percentage of Gram positive bacteria, percentage of rods and numbers of polymorphonuclear neutrophils (PMNs) by one experienced examiner (J.J.S.). Clumps were excluded from assessment. The surface area of red fluorescence on two right upper molars (16 and 17) was determined by dedicated software (QA1, version 1.0.0.5, Inspektor Research Systems). No significant correlations were found between red fluorescence (percentage) and percentage Gram positive bacteria $\left(r_{s}=-0.081, p=0.4\right)$, percentage of rods $\left(r_{s}=0.036, p=0.7\right)$, and amount of PMNs $(\mathrm{r}=-0.050, \mathrm{p}=0.6)$. We conclude that neither the percentage of Gram positive bacteria, the percentage of rods, or the number of PMNs, are significantly associated with the percentage of red fluorescence.

This research is supported by the Dutch Technology Foundation STW (project number 10948) and Inspektor Research BV.

\section{9 \\ Salivary Mutans Streptococci Levels with Different Fluoride-Releasing Restorative Materials}

\author{
M.E.S. ElBanna*, I.M.G. Elzayat \\ salah_mai@yahoo.com \\ Operative Department, Misr International University, Cairo, \\ Egypt
}

The aim of this study was to evaluate the effect of different fluoride-releasing restorative materials on the mutans streptococci levels in saliva. A total of thirty patients were selected for this study and written informed consents were obtained from every patient. Patients included in this study required the restoration of eight cavities performed at the same day on two long clinical sessions; they showed the same average baseline mutans streptococci growth of score 2 or 3 which was measured for all patients using Dentocult SM kit. Patients were divided into three groups of ten patients each according to the type of the fluoride-releasing restoration received. Group 1: conventional fluoride-releasing glass ionomer (Dentsply); group 2: Ketac ${ }^{\mathrm{TM}}$ Fil Plus Aplicap Glass ionomer restoration (3M); group 3 received Beautifil II Giomer (Shufo), placed using Flurobond, restoration. The count of mutans streptococci in saliva for every patient in the three groups was assessed at day 2 using the Dentocult SM strips. The same assessment for the mutans streptococcus count in saliva was done at week 2 and after one month. The mutans streptococcus count in saliva was evaluated using the manufacturers' chart. The mutans streptococcus levels in saliva were analysed using Chi-square test and showed significant reduction in both group 1 and 3 as the average score was ranged form $0-1$ on day 2 but there was no significant difference between them. The mutans streptococcus level in saliva in group 2 showed inferior results as the average score was 2 . Same differences were obtained after two weeks and one month. In conclusion Beautifil II exhibited the same antibacterial effect on salivary levels of mutans streptococci as the conventional glass ionomer.

Misr International University. 


\section{Caries Research}

\section{Session 8 Caries Risk Assessment, Dental Plaque}

\section{0 \\ Salivary Mutans Streptococci in Mothers: A Predictive Risk Factor for Caries in Their Children? \\ T.T. Basso ${ }^{\mathrm{a}, \mathrm{c}}$, G. Campus ${ }^{\mathrm{b}, \mathrm{c}, *}$, M.G. Cagetti ${ }^{\mathrm{c}}$, A. Senna ${ }^{\mathrm{c}}$, C. Galeone $^{\mathrm{c}}$, L. Strohmenger ${ }^{c}, P$. Lingström ${ }^{d}$ \\ gcampus@uniss.it \\ aTolmezzo General Hospital, ' Dental Institute, University of Sassari, and 'WHO Collaborating Centre of Milan for Epidemiology and Community Dentistry, Italy; ${ }^{\mathrm{d} D e p a r t m e n t}$ of Cariology, Institute of Odontology at Sahlgrenska Academy, University of Gothenburg, Gothenburg, Sweden}

The aim of this study was to assess the relationships of quantitative salivary levels of mutans streptococci (ms) recorded in mothers immediately after delivery and early childhood caries (ECC) in the children at 3 years of age. A retrospective study was designed. In an Italian north-eastern county a preventive programme against caries in children started in 2006. This included the recording of ms levels in mothers. In spring 2010, 5.7\% (n = 496) of the cohort of children born in 2006 ( $n=8,747$ subjects) was examined. dmfs index was recorded at D1-D3 level. Behavioural and socio-economical variables were registered using a standardized questionnaire self-compiled by parents. ECC was observed in $28.6 \%$ of the children. Mothers were divided into two groups regarding salivary ms levels: low level $\left(<10^{5} \mathrm{CFU} / \mathrm{ml}\right)$ $63.6 \%$ and high level $\left(>10^{5} \mathrm{CFU} / \mathrm{ml}\right) 36.4 \%$. Mean (SE) dmfs was higher in children whose mothers had a high ms level: $2.66(0.61)$ versus $1.78(0.26)$, but this difference was not statistically significant $(\mathrm{F}=2.36 \mathrm{p}=0.13)$. dmfs was highly skewed (Fisher $\left.\chi^{2}=2.86\right)$, so the index was categorized into 3 classes. Association among ECC and several background factors was found e.g. gender, use of pacifier with sugar at night, educational level of the mother, maternal smoking habits and language spoken at home. Zero-inflated negative binomial regression modelling was used with the cat- egorization of $\mathrm{dmfs}$ as dependent variable. The probability to have an extra zero in the categorization of dmfs was linked to mother's nationality $(p<0.01)$, use of pacifier with sugar at night $(p=0.04)$ and educational level of the mother $(\mathrm{p}=0.01)$. In conclusion, this study failed to associate mother salivary $\mathrm{ms}$ level as a predictive caries risk factor in children.

\author{
141 \\ Customized Oral Health Information Using Tooth \\ Life Curves \\ B.I. Kim*, S.H. Jung, H.K. Kwon \\ drkbi@yuhs.ac \\ Department of Preventive Dentistry \& Public Oral Health, \\ Research Center for Orofacial Hard Tissue Regeneration, Brain \\ Korea 21 Project, College of Dentistry, Yonsei University, Seoul, \\ South Korea
}

Most oral health indicators have been developed based on diseased oriented concept focusing on dental caries and periodontal disease. Individuals are generally classified a having or not having disease by a clinical diagnosis. However, this dichotomous classification has limitations in expressing the customized oral health status of individuals. The aim of this study was to develop a new model of tooth life curves to present the customized oral health status graphically. For this, we adopted the concept of percentile curves which are already used for growth and development curves in the pediatric field. This study used the Korean national oral health survey data in 2006 . There were a total of 4,546 subjects over 18 years of age. The main variables used for the tooth life curves were the number of teeth present (PT), sound teeth (ST) and filled-sound teeth (FST) according to age and gender. This 
study selected 5 representative percentile values such as 10 th, 25 th, 50 th, 75 th and 90 th. The 10 th and 25 th groups indicated the good oral health groups. The 75th and 90th groups classified the poor oral health groups. PT curves is applicable to the elderly population because it is difficult to distinguish between the good and poor oral health group in the young population. ST curves showed distinct differentiation both young and old populations. However, there is a limitation which ST cannot reflect directly the advisable changes to oral health behaviors. FST curves can distinguish a good and poor oral health group in both young and old populations. In conclusion, tooth life curves can suggest the individual relative position of the oral health status among the spectrum using a graph.

This research was supported by Basic Science Research Program through the National Research Foundation of Korea (NRF) funded by the Ministry of Education, Science and Technology (R13-2003-013-05002-0).

\section{2}

\section{Is Dental Decay Related to Child Neglect?}

A.P.G.F. Vieira*, C. Lourenço, M.V. Santrain

anyavieira10@gmail.com

Public Health Master Program, Universidade de Fortaleza, Brazil

Background: Despite all the public health policies, dental decay continuous to be one of the most prevalent diseases in children. Among the factors related to dental decay in children is the lack of care from caregivers towards their children, which can be seen as neglect. However, not much is known regarding the relationship between dental decay and neglect, which is the aim of this research. Methods: Five-year-old children in a small city ( $\mathrm{Pa}-$ coti, Ceará, Brazil) with public and well organized dental care assistance were evaluated for dental problems, utilizing the $\mathrm{dmft}$ index (number of decayed, missing, or filled teeth), and for signs of neglect (general hygiene: dental, hair, fingernail hygiene - e.g., long or dirty fingernails, hair lice, unbrushed or tangled hair, high quantity of dental plaque), through a visual inspection by the same investigator. Results: All 5-year-old children from the municipality were searched for, and a total of 149 children (with parents' and children's consent), from all socio-economic level, were examined. Only $34.9 \%$ were caries free, and more than $20 \%$ had 5 or more cavities. The mean dmft was 2.75 (SD 2.84), where the majority of the teeth were cavitated (average of 2.2 per person). $32.9 \%$ had dmft of 4 or more. $64.5 \%$ presented bad or partially bad oral hygiene. There was a strong and significant relationship between bad oral hygiene and dental decay experience $(\mathrm{dmft})(\mathrm{p}=$ 0.001 ). There was also a relationship between general hygiene and hair and fingernail hygiene $(\mathrm{p}<0.005)$. Conclusion: The finding suggest that children that are not properly taken care of (that have been neglect on their general hygiene) are more prone to have dental decay.

We would like to thank the CNPq (Brazilian National Research Council) for the funding of this research.

\section{3 \\ Correlation among Gold Standard Techniques in Validating Methods for the Detection of Occlusal Caries}

J.A. Rodrigues ${ }^{\mathrm{a}, *}$, K.W. Neuhaus ${ }^{\mathrm{c}}$, M.B. Diniz ${ }^{\mathrm{b}}$, I. Hug ${ }^{\mathrm{c}}$, H. Stich', L. Karlsson ${ }^{\text {d }}$, A. Lussi ${ }^{\mathrm{C}}$

jorodrigues@hotmail.com

aFaculty of Dentistry, Federal University of Rio Grande do Sul, and ${ }^{\mathrm{b} C}$ ruzeiro do Sul University, São Paulo; ' ${ }^{\mathrm{S}}$ chool of Dental

Medicine, University of Bern, Switzerland; dDepartment of

Dental Medicine, Karolinska Institutet, Huddinge, Sweden

The aim of this in vitro study was to assess the correlation among four techniques used as gold standard for the validation of methods for occlusal caries detection. Sixty-five human permanent molars were selected and one site in each occlusal surface was chosen as the test site. First, a groove was made on the teeth in order to allow orientation of the test surfaces. The teeth were cut and consecutively prepared according to each of the following techniques: stereomicroscopy without colouring (1), dye enhancement with rhodamine $\mathrm{B}(2)$, dye enhancement with fuchsine acid light green (after cleaning the specimen from rhodamine $\mathrm{B}$ and after obtaining a thin slice of $300 \mu \mathrm{m}$ thickness) (3), and semiquantitative microradiography (4). Digital photographs taken from each specimen were assessed by three examiners for caries extension according to the Downer classification. In case of disagreement a consensus was reached. Weighted kappa and Friedman's test with multiple comparisons were performed in order to compare all techniques and verify statistically significant differences. Kappa values varied from 0.62 to 0.78 , the latter being found in both dye enhancement methods. Friedman's test showed statistically significant differences $(\mathrm{p}<0.001)$ and multiple comparison indentified these differences among all techniques, except between both dye enhancement methods (rhodamine B and fuchsine). Cross-tabulation showed that stereomicroscopy tended to overscore D2 lesions in particular when compared to all other methods. The smallest amount of overscoring in comparison to other methods was observed for microradiography. Therefore, the outcome of caries diagnostic tests may be influenced by the validation method used as reference standard. Dye enhancement methods seem to be reliable as histological gold standard.

University of Bern, Switzerland and Karolinska Institutet, Huddinge, Sweden.

\section{4 \\ Risk Assessment of Dental Caries in Adult Patients Using Multiple Logistic Regression Analysis}

\author{
M. Hayashi*, A. Ito, S. Ebisu \\ mikarin@dent.osaka-u.ac.jp \\ Department of Restorative Dentistry and Endodontology, \\ Osaka University Graduate School of Dentistry, Japan
}

Being able to predict an individual's risk of dental caries would offer a huge natural step forward in promoting oral health. Currently, preventative dental treatment is mostly carried out without 
caries risk assessment because there is little way of knowing about an individual's risk factors. The aim of this study was to use multiple logistic regression analysis to try to identify the significant factors affecting the onset of new caries lesions in adult patients. In this historical cohort study, a data set of 442 patients from a single general practice who met the inclusion criteria was analyzed. Multiple logistic regression analyses to assess caries risk were performed with the following parameters for each patient: age, number of carious teeth, levels of cariogenic bacteria, the secretion rate and buffer capacity of saliva and compliance with a prevention program. The odds ratios for the onset of caries for each variable were calculated, and those within three years and during three and five-year time were compared. The levels of mutans streptococci (MS) affected the onset of primary and secondary caries within three years with odds ratios of 2.34 (95\% CI: $1.15-4.76, \mathrm{p}=0.0191)$ and $2.22(95 \% \mathrm{CI}: 1.06-4.62, \mathrm{p}=0.0339)$, respectively. The levels of lactobacilli (LB) affected the onset of secondary caries within three years with the odds ratios of 3.34 (95\% CI: $1.35-6.85, \mathrm{p}=0.0072$ ), but LB did not affect the onset of primary caries (odds ratio: $1.68,95 \% \mathrm{CI}: 0.87-3.25, \mathrm{p}=0.1202$ ). The significant influences of both MS and LB on the onset of primary and secondary caries were not detected after three years. In conclusion, cariogenic bacteria play an important role in the incidence of caries, but the influence of these bacteria was not detected after three years.

This study was supported by Grants-in-Aid for Scientific Research (Nos. 20249076 and 22390358) from the Japan Society for the Promotion of Science.

\section{5 \\ Biological and Social Determinants of Early Childhood Caries}

\begin{abstract}
S. Skrivele ${ }^{\mathrm{a}, *}$, S. Berzina a , R. Care ${ }^{\mathrm{a}}$, E. Maslak ${ }^{\mathrm{b}}$, T. Tserekhava ${ }^{\mathrm{c}}$, N. Shakovets ${ }^{\mathrm{c}}$, R. de Moura-Sieber ${ }^{\mathrm{d}}$, V. de Moura ${ }^{\mathrm{d}}$, M. Wagner ${ }^{\mathrm{e}}$, S. Kneist ${ }^{\mathrm{d}}$, A. Borutta ${ }^{\mathrm{d}}$

simonaskrivele@e-apollo.lv

${ }^{a}$ Department of Conservative Dentistry, Riga Stradins University, Riga, Latvia; ${ }^{\text {b}}$ Department of Pediatric Dentistry, State University of Volgograd, Russia; ' Department of Pediatric Dentistry, State Medical University of Belarus, Belarus; ${ }^{\text {WHO Collaborating }}$ Center on Prevention of Oral Diseases at Centre for Dental, Oral and Maxillofacial Surgery, University Hospital of Jena, and eDepartment of Economic and Social Statistics, Friedrich Schiller University of Jena, Germany
\end{abstract}

Between 2002 and 2008, the WHOCC on Prevention of Oral Diseases at Jena University Hospital initiated a multi-centre study (Brazil, Germany, Latvia, Russia and Belarus) in order to elucidate an explanatory model of early childhood caries (ECC). 472 infants aged 26 to 34 months were included. A uniform study design with a health survey of mothers and oral examination of infants (plaque on the upper incisors, $\mathrm{dmft}$ ) was used. The data were evaluated descriptively (SPSS, 15.0) and graphically (TETRAD-IV, PC algorithm) to identify direct and indirect connections between the factors of influence and $\mathrm{dmft}$. The relations were quantified by the correlation coefficient $(r)$. The variable country had a direct influence on the cleaning control $(\mathrm{r}=-0.08)$, the work of the mother $(r=0.18)$, the teeth-cleaning of the infant $(=-0.26)$, use of the bottle $(\mathrm{r}=-0.23)$ at night $(\mathrm{r}=-0.16)$, plaque score $(\mathrm{r}=0.16)$ and $\mathrm{dmft}(\mathrm{r}=-0.11)$. The higher was the standard of living of the country, the more often the teeth were cleaned, the cleaning was controlled and less plaque was scored. The bottle was given more often $(r=-0.23)$; also at night. Between the variables direct relations existed. If the mother was working, the infant got less the bottle before falling asleep. If mother had a good education, the dental cleaning of the infant was controlled more often. If the infant received the bottle, it also had the bottle before falling asleep $(\mathrm{r}=0.31)$ and at night $(\mathrm{r}=0.2)$. The night bottle was associated with raised the plaque score on the upper incisors $(r=0.17)$ and $\mathrm{dmft}(\mathrm{r}=-0.21)$. For prevention of ECC the controlled use of the bottle and the use of unsweetened drinks is necessary. The use of the cup from 9 month of life and regular dental and oral hygiene with age-appropriate $\mathrm{F}$ toothpaste promotes the dental health of infants.

\section{6 \\ Effectiveness of Integrated Non-Operative Caries Treatment on Risk Profiles and Root Caries in High-Risk Adults}

N. Grguraš, M. Mastnak, J. Jan*

janja.jan@mf.uni-lj.si

Department of Dental Diseases and Endodontics, University of

Ljubljana, Slovenia

The aim of this pilot study was to evaluate the effectiveness of integrated non-operative caries treatment on caries risk profiles using the Cariogram model and active root caries progression in high-risk adults. Forty-three 32- to 67-year-old patients, with high caries risk and at least two active root caries lesions were randomized into either a control group that received standard dental care with oral hygiene instructions and dietary recommendations, or to a test group that additionally received fluoride varnish applications, fluoride tablets, $0.12 \%$ chlorhexidine rinse, and personalized treatment planning and recalls. At baseline, information on Decayed-Filled-Root-Surfaces (DFRS), diet, oral hygiene and use of fluoride had been obtained. Saliva analyses included mutans streptococci and lactobacilli, buffer capacity and secretion rate. The Cariogram was used to evaluate the risk profiles. Root caries lesions were classified utilizing ICDAS criteria. Lesion activity was determined and active and inactive lesion surface areas were measured with a periodontal probe. Outcome variables were recorded at baseline and after 6 months. When the two groups were compared based on DFRS, the values at baseline (test group: $7.06 \pm 3.09$; control group: $5.00 \pm 2.18$ ) and after 6 months (test group: $7.25 \pm 3.30$; control group: $6.83 \pm 2.39$ ) were not significantly different $(\mathrm{p}>0.05)$. The mean Cariogram chance of avoiding caries significantly $(\mathrm{p}<0.01$, Wilcoxon test) improved from $5.92 \pm 3.46 \%$ to $34.69 \pm 14.33 \%$ in the test group, and from $6.77 \pm 6.91 \%$ to $7.15 \pm 7.44 \%$ in the control group $(p>0.05)$; the chance was significantly different between the two groups after 6 months $(p<0.01)$, but not at baseline $(p>0.05)$. The mean active areas of root caries lesions significantly $(\mathrm{p}<0.01)$ decreased in the 
test group from $53.09 \pm 12.28$ to $9.12 \pm 6.52 \mathrm{~mm}^{2}$, and increased from $41.03 \pm 10.96$ to $56.73 \pm 8.16 \mathrm{~mm}^{2}$ in the control group. After 6 months the active areas values were significantly higher in the control group compared to the test group $(p<0.01)$, at baseline they were higher in the test group $(\mathrm{p}<0.05)$. The results showed that integrated non-operative preventive treatment can lower caries risk and root caries progression in high-risk adults.

Supported by Slovenian Ministry of Science and Education (No. P3-0374).

147

\section{Impact of Enamel Defects on Early Caries Development in Preschool Children}

H.D. Mestrinho ${ }^{a}$ * , E.F. Silva ${ }^{\text {b }}$, J.A.C. Fonseca ${ }^{\mathrm{a}}$, R.R.C. Gomes ${ }^{\mathrm{a}}$, J.C. Carvalho ${ }^{a, c}$

joana.carvalho@uclovain.be

aFaculty of Health Sciences and bDepartment of Statistics, University of Brasília, Brazil; ' $F$ Faculty of Medicine and Dentistry, Catholic University of Louvain, Brussels, Belgium

Quantitative defects of the enamel are considered risk factors for caries development at cavitated level. Since caries risk assessment and control should be implemented as early as possible in order to prevent operative treatment, it seems interesting to investigate the relationship between enamel defects and caries development also in stages of progression which precede cavitation. The impact of enamel defects on early caries development was investigated in a cohort of Brazilian preschool children. The null hypothesis that developmental defects of the enamel and dental caries are independent and that an association between them occurs by chance was tested. The sample $(n=1,718)$ was formed by 2 - to 5 -year-olds attending public nursery in the Federal District of Brazil ( 0.8 ppm F). Before the examination the children were laid on tables and had their teeth professionally brushed, flossed and dried by means of gauze bandages. Intra- and inter-examiner reliability of developmental defects and caries diagnosis showed non-weighted kappa values of $0.82-0.88$. Tooth surfaces were diagnosed as sound, as presenting active lesions (non cavitated and cavitated), inactive lesions (non-cavitated and cavitated) and fillings, as indicated for extraction or extracted. The buccal surfaces were examined for presence of demarcated or diffuse opacity, hypoplasia and their different combinations. Developmental defects of enamel and caries on buccal surfaces were identified in 48 and $26 \%$ of the children, respectively. Bivariate analyses at surface level neither showed association between demarcated/diffuse opacity and caries experience nor between hypoplastic surfaces and non-cavitated lesions $(\mathrm{p}=0.29, \mathrm{GLM})$. In the multivariate analyses, within-child hypoplastic surfaces were significantly associated with fillings, non-cavitated and cavitated lesions ( $p \leq 0.03$, GEE), but children having enamel hypoplasia were not at higher caries risk than those without it ( $\mathrm{p}=0.23$, GEE). The null hypothesis could not be accepted for quantitative defects such as hypoplasia, since it had a significant impact on the prevalence of within-child filled surfaces, noncavitated and cavitated lesions.

\section{8}

\section{A Comparative Study for Caries Prediction from 18- to 36-Month-Old Children}

\author{
Y. Maki ${ }^{\mathrm{a}, *}$, A. Kurokawa ${ }^{\mathrm{b}}$, K. Yamada $^{\mathrm{b}}$ \\ maki@tdc.ac.jp \\ aDepartment of Social Dentistry, Tokyo Dental College, Chiba \\ and ${ }^{\text {b}}$ The Lion Foundation for Dental Health, Tokyo, Japan
}

A semi-quantitative enumeration system was developed to detect Streptococcus mutans in saliva. This system generates a result of 3 levels of $S$. mutans within $30 \mathrm{~min}$. The aim of present study was to explore the feasibility of predicting caries incidence from 18- to 36-month-old using this system. At the same time, another microbial test, environmental factors and oral health behavior were introduced for the comparison. 247 children at the age of 18 months and their parents participated in this study. Saliva samples were collected from children and parents using a swab technique. These saliva samples were applied into the semi-quantitative $S$. mutans test device using monoclonal antibodies (SALIVACHECK MUTANS; GC, Japan) and Resazurin Disc Test (Showa yakuhin kako, Japan) for the detection of total microorganisms. At the same time, caries experience was examined and questionnaire for oral health behavior was carried out. After 18 months, the same caries risk tests and the caries examination was repeated. Caries incidence and caries increment were calculated for each caries risk level and oral health behavior. The caries increment at the $S$. $m u$ tans test level 1, 2 and 3 were 1.2, 1.4 and 3.6, respectively. There was a significant difference between level 1 and $3(\mathrm{p}<0.05)$. Caries prevalence at the age of 18 months was significantly related to the caries increment after 18 months $(p<0.05)$. Similar level of correlation was found for $S$. mutans and total microorganisms levels between children and parents. However, levels of total microorganisms and oral health behaviour was not related to the caries increment. These results suggest that level of $S$. mutans and caries experience could predict the caries incidence after 18 months.

\section{9 \\ Quickscan-Measured Self-Assessed Parental Attitudes and Caries Risk-Related Behaviour in 6-Year-Old Children}

\author{
J.H. Vermaire ${ }^{a}, *$, C. van Loveren ${ }^{b}$, J. Hoogstraten $^{a}$ \\ e.vermaire@acta.nl \\ aDepartment of Social Dentistry and Behavioural Sciences, \\ ACTA Amsterdam, The Netherlands; ${ }^{b}$ Department of \\ Experimental Preventive Dentistry, Clinical Cariology and \\ Microbiology, ACTA Amsterdam, The Netherlands
}

Parental attitudes were determined using a self-assessed attitude tool, making them choose between textual descriptions of attitudes. These descriptions were based on a Q-methodological study [Vermaire et al: Community Dent Oral Epidemiol 2010;38: 507-520]. This tool distinguishes 5 parental attitudes towards oral health: (1) conscious and responsible: aware of the need to care and willing to take the responsibility, (2) trivializing and fatalistic: 
convinced that oral health is a genetic matter and that caries is not a major problem, (3) appearance-driven and open-minded: following the dental professional's advice because having good looking teeth is considered important, (4) knowledgeable but defensive: aware of why and how to apply oral care but also report to be too busy to do so, and (5) conscious and concerned: afraid that all efforts in maintaining oral care will be idle at the end. Clinical data (dmfs, plaque) were collected by two calibrated dentists and nonclinical data (SES, willingness to pay, motivational issues) were collected using questionnaires. Data of 145 participants were compared between these typologies using ANOVA (parametric data) and Kruskal-Wallis tests (non-parametric data). Parents with attitude 3 had highest scores on Willingness to Pay $(\mathrm{F}=2.44, \mathrm{p}<$ $0.05)$. Parents with attitude 2 valued general health and oral health lower compared to other attitudes $(\mathrm{F}=2.46, \mathrm{p}<0.05)$ and were less likely to have additional dental insurance $\left(\chi^{2}=10.65, \mathrm{p}<0.05\right)$. Parents of attitude 3 and 5 reported that their child eat more regular meals and less between-meal snacks, compared to other attitudes, especially attitude $2\left(\chi^{2}=9.90, \mathrm{p}<0.05\right)$. The lowest percentage of caries-free children was found in children of parents having attitude 4 (32\%); highest percentage of caries-free children was found in attitude 5 (67\%). The self-reported attitudes were associated with different health behaviours and with caries related risk factors but not with caries prevalence.

\section{0 \\ Relevant Caries Risk Factor in Children: A Case/ Control Study in Villavicencio, Colombia 2010}

N. Chavarra ${ }^{\mathrm{a}}$ b, * , L. Duran ${ }^{\mathrm{a}}$, K. Duran ${ }^{\mathrm{a}}$, S.M.Gordillo ${ }^{\mathrm{a}}$, K.R. Ekstrand

nathychbol@yahoo.com

aDental Faculty, Universidad Cooperativa de Colombia, Sede

Villavicencio, and 'buniversidad de los Llanos, Villavicencio,

Colombia; ' ${ }^{\circ}$ niversity of Copenhagen, Denmark

Recent data show that the dmf-t level in Colombia among 5 -year-olds is 3 . This pilot study investigates if there are any risk indicators, which predict children in Colombia to be in high versus low risk of getting caries. The risk indicators evaluated were 9 of the 10 indicators stated in the interactive Cariogram program. In this study they were: Related diseases, Diet content, Diet frequency, Plaque occurrence, Mutans streptococcus, Fluoride program, Saliva flow, Buffer capacity, and finally the clinical judgment of the examiner involving an overall assessment of the caries risk for that individual child. A total of 105 children from Villavicencio, Colombia were invited to take part in the study, of which we received informed consent from 68 children/parents. They were divided into controls $(\mathrm{n}=34)$ and cases $(\mathrm{n}=34)$ based on a dmft $<3$ or $\geq 3 \mathrm{dmft}$. The mean dmft in the control group was $2.2 \pm 0.7$ versus $4.2 \pm 0.9$ in the case group. The children were then characterized by means of the risk indicators in the Cariogram, where each indicator was dichotomized indicating low risk or high risk. Odds ratio was used to express the association between each indicator and caries experience of the children. The odds ratio (OR) and their $95 \%$ confidence interval $(95 \% \mathrm{CI})$ were as follows: $\mathrm{Re}-$ lated diseases OR = 1 (95\% CI = 0.1-7.5); Diet content OR = $1(95 \%$ $\mathrm{CI}=0.3-3.2)$; Diet frequency $\mathrm{OR}=2.9(95 \% \mathrm{CI}=1.0-8.8)$; Plaque occurrence $\mathrm{OR}=4.6(95 \% \mathrm{CI}=3.1-6.3)$; Mutans streptococcus $\mathrm{OR}=1.3(95 \% \mathrm{CI}=0.5-3.6)$; Fluoride program OR = $1(95 \% \mathrm{CI}=$ $0.1-7.5)$; Saliva flow OR $=1$ (95\% CI $=0.05-0.66)$; Buffer capacity $\mathrm{OR}=1.43(95 \% \mathrm{CI}=0.2-2.3)$, and the clinical judge $\mathrm{OR}=3.3(95 \%$ CI 1.1-5.2). From the data it can be seen that frequency of diet, plaque occurrence, and clinical judgment is strongly (lower value in the $C L \geq 1$ ) associated with those children with high caries experience. If this knowledge can be confirmed in larger studies, it means that the interactive Cariogram, which free of charge can be downloaded, can be used in Colombia without involving the expensive laboratory tests when assessing caries risk on children.

No external funding.

\section{1 \\ Comparison of the Cariogenic Traits between Xylitol-Resistant Mutans Streptococci Exposed to Various Carbohydrates}

S.Y. Chung*, Y.S. Jung, Y.H. Choi, K.B.Song

kbsong@knu.ac.kr

Department of Preventive Dentistry, School of Dentistry, Kyungpook National University, Republic of Korea

Objective: The purpose of this study was to compare the cariogenic traits of xylitol-resistant (XR) Streptococcus mutans and XR S. sobrinus exposed to various carbohydrates. Methods: To select XR Mutans Streptococci (MS) bacterial cells, S. mutans KCTC 3065 and S. sobrinus KCTC 3308 were cultured in a TYE medium containing $0.5 \%$ glucose and $1 \%(66 \mathrm{mM})$ xylitol at $37^{\circ} \mathrm{C}$, $10 \% \mathrm{CO}_{2}$ conditions for 30 days. The organisms of both strains were divided into the following 4 groups on the basis of the carbohydrate sources: (1) $0.5 \%$ glucose contained basal culture medium (BCM), (2) BCM plus 0.5\% sucrose (BCM-S), (3) BCM plus $0.5 \%$ fructose (BCM-F), and (4) BCM plus $0.5 \%$ maltose (BCM$\mathrm{M})$. To identify the cariogenic traits, cell growth, acid production, mRNA expressions related to glucans synthesis ( $g t f B$ and $g t f D$ for S. mutans; $g t f I$ and $g t f U$ for S. sobrinus), and the formation of extracellular polysaccharides (ECPs) were observed. Kruskal-Wallis test and Tukey post hoc analysis assessed the difference according to the carbohydrate source. Results: XR $S$. mutans grew higher in BCM-M, yet lower in BCM-S, -F compared to BCM. However, final growth of XR $S$. sobrinus not significantly differed except BCM-S. Both strains expressed the final $\mathrm{pH}(4.20-4.29)$ without any significant differences depending on dietary sugar sources. The relative levels of mRNA expression in $g t f B$ and $g t f D$ were higher in BCM-S (fold changes, 1.36-1.50), BCM-F (1.49-1.51) compared to BCM (1.00), while those in $g t f I$ and $g t f U$ were only higher in BCM-S (1.4-1.64) than BCM (1.00). ECPs formation was apparently distinct in $S$. mutans depending on sugar sources whereas those were not different in S. sobrinus except BCM-S. Conclusion: The cariogenic traits of XR S. mutans responded differently when grown in mixed carbohydrates, yet those of XR $S$. sobrinus, in general, showed no difference depending on sugar sources except BCM-S. These differences might be related to the different cariogenic traits between $S$. mutans and S. sobrinus.

This study was partially supported by Korea Caries Prevention Association Research Fund, 2009. 


\section{2 \\ Explanatory Study Testing Digit Ratio Theory and Caries Experience Correlation in High-Risk Cree Children}

J. Véronneau*

jacques.veronneau@mcgill.ca

Faculty of Dentistry, McGill University, Montreal, Canada

Assessing a patient's risk of developing caries is a vital component of caries management. A comprehensive caries assessment should consider also physical factors. An emerging theory [Manning JT: Digit Ratio: A Pointer to Fertility, Behavior and Health. New Brunswick, NJ, Rutgers University Press, 2002] is the digit (D) ratio - ratio of the length between the ring (D4) and index (D2) finger - showed as correlated to health problem predisposition. The aim of this study was to assess the correlation between digit ratio and caries experience assessed with the ICDAS system on Cree Indian children. Forty-eight children and adolescents attending Cree schools in Quebec were examined for caries experiences, completed a caries-prevention questionnaire and had their right-hand photocopied. The ICDAS system was used by one trained and calibrated dentist for caries evaluation and measured using portable equipment. Digit ratio was measured using a photocopied image by an evaluator blinded to the caries experience of the children. Ratio association assessments have been tested using multiple regression models. Mean age of children involved was 11.4 years $( \pm 2.9)$. The DMFT - number of teeth per child, with carious lesions or filling or tooth's missing for caries - was in average, 11.4 teeth $( \pm 6.0)$ per child. The sample included $53 \%$ of boys. Weighted kappa values for intra-examiner digit measurements reproducibility were $0.92-0.98$ and differences were not significant $(p>0.05)$. The mean digit ratio was $1.07(6.75 \mathrm{D} 4 / 6.25$ D2) $\mathrm{cm}( \pm 0.06)$ with $1.07 \mathrm{~cm}$ for girls and $1.09 \mathrm{~cm}$ for boys. Backward and forward method on model of regression did not show significant correlation between digit ratio and DMFT. Ratio digit measurement with fingers suggests in that specific sample, no evidence of significant association with dental caries experience.

Cree Health Board, James Bay, Quebec, Canada.

\section{3 \\ The Consumption of Non-Milk Extrinsic Sugars and Dental Caries Risk in Adolescents

\author{
M.G. McGrady ${ }^{\text {a, * }}$, R.P. Ellwood ${ }^{\mathrm{a}}$, I.A. Pretty ${ }^{\mathrm{a}}$, A. Maguire ${ }^{\mathrm{b}}$, \\ M. Goodwin a D. Patel ${ }^{\mathrm{a}}$ \\ michael.mcgrady@manchester.ac.uk \\ aSchool of Dentistry, University of Manchester, and ${ }^{b}$ Centre for \\ Oral Health Research, Newcastle University, UK
}

Clinical care pathways encompass caries risk assessment and the role of dietary non-milk extrinsic sugars (NMES) to help inform preventive and intervention strategies. The aim of this study was to investigate the effect of dietary habits on caries experience. The frequency and timing of NMES consumption including snacking between meals and bedtime consumption were investi- gated to determine if any factors could be utilized to predict caries risk. Subjects were randomly selected from an observational epidemiological survey for which the caries status and level of material deprivation was available. Subjects underwent a $24 \mathrm{~h}$ dietary recall interview with a dietician focussing on NMES intake. The estimation of NMES intake was based on McCance and Widdowson's food composition tables. In total 128 subjects (aged 12-13) were included in the analysis. $54(42 \%)$ subjects were found to have caries (DMFT based on ICDAS code $\geq 4$ ). Chi-square analysis for subject groups with or without caries revealed no significant differences in caries experience between high and low deprivation, consumption of NMES between meals and percentage of total NMES consumed. The consumption of NMES before bedtime revealed a statistically significant difference between the caries/no caries groups $(\mathrm{p}<0.005)$. Logistic regression analysis on the caries/no caries groups revealed a predictive odds ratio of 2.26 $(1.23,4.15)$ for NMES consumption before bedtime that correctly predicted $65 \%$ of caries experience (when age of exam included as a variable). However, a small effect size was noted possibly owing to the relatively low caries experience overall and small sample size. The study suggests that the consumption of NMES before bedtime may be an important predictive risk factor for adolescent caries experience. Further work is necessary to validate the findings of this study.

Dr. Pretty is funded by a Clinician Scientist Award from the National Institute for Health Research (UK). This study was supported by the Colgate Palmolive Dental Heath Unit, Manchester, UK.

\section{4 \\ Quantitative Evaluation of Plaque Biofilm Removing Capacity of a Dental Water Jet Using Electron Probe Micro-Analyzer}

\author{
K. Kato*, K. Tamura, H. Nakagaki
}

kazkato@dpc.aichi-gakuin.ac.jp

School of Dentistry, Aichi-Gakuin University, Nagoya, Japan

This study was carried out to evaluate plaque biofilm removing capacity of a dental water jet (DWJ) by measuring biofilm thickness using an Electron Probe Micro-Analyzer (EPMA). Thirty consenting subjects (18-35 years) wore in situ plaque-generating devices, which consisted of a pair of $4 \mathrm{~mm}^{2}$ enamel slabs attached to the upper molars for 2 days. Each device removed from the mouth was clamped, and one of slab surfaces was treated with the DWJ (Dentrex, Ricoh Elmex Corporation), irrigating for $5 \mathrm{~s}$ at a distance of $3 \mathrm{~mm}$ away from the surface. The devices were randomly assigned to three different pressure settings of 707, 350 or $102 \mathrm{kPa}$. Another slab with no treatment served as control. Each slab was freeze-dried, sputter-coated with platinum, and examined using secondary-electron imaging. The slabs were then embedded in methacrylate and cross-sectioned in the middle. Their surfaces were polished, coated with carbon, and examined using backscattered electron compositional (COMPO) imaging. The area between the enamel and the outer biofilm surface indicated by thin platinum layer was measured in the COMPO image to calculate the average thickness of the biofilm on the specimen. 
Removing capacity of biofilm by irrigation was estimated using the reduced rate of biofilm thickness, which was calculated from the differences between a pair of treated and control slabs. The biofilm thickness (mean \pm SD) of control slabs was $16.5 \pm 13.6$ $\mu \mathrm{m}$. The reduced rates were $85.5 \pm 11.4 \%$ at $707 \mathrm{kPa}(\mathrm{p}<0.05)$, $85.1 \pm 7.2$ at $350 \mathrm{kPa}(\mathrm{p}<0.001)$ and $63.4 \pm 13.7$ at $102 \mathrm{kPa}(\mathrm{p}<$ 0.05 ), indicating that the biofilm thickness was significantly reduced at every pressure setting. The results suggested that the irrigation using DWJ would be an effective means of plaque control.

Supported by KAKENHI (C) No. 20592477, Japan.

\section{5}

\section{Evaluation of the Anticariogenic Potential of Adhesive Systems with a Microcosm Biofilm Model}

F.H. van de Sande ${ }^{\mathrm{a}, *}$, M.S. Azevedo ${ }^{\mathrm{a}}$, R.G. Lund ${ }^{\mathrm{a}}$, M.C.D.N.J.M. Huysmans ${ }^{\mathrm{b}}$, M.S. Cenci ${ }^{\mathrm{a}}$

fvandesande@gmail.com

aschool of Dentistry, Federal University of Pelotas, Pelotas, Brazil; bCollege of Dental Science, Radboud University

Nijmegen Medical Center, Nijmegen, The Netherlands

Antimicrobial monomers (AM) have been incorporated into adhesive systems with the aim of reducing the development of secondary caries. This study evaluated the anticariogenic potential of adhesive systems, with and without AM, on adjacent enamel surfaces. Enamel discs ( $n=8$ per group) obtained from bovine incisors were prepared with standardized cavities and randomly restored with composite resin using three adhesive systems; a conventional three step adhesive system (Single Bond 2, 3M ESPE; negative control), a two step self-etch system with AM (Clearfil Protect Bond, Kuraray), and a two step self-etch system (Clearfil SE Bond, Kuraray). Eight discs were also restored with a resinmodified glass ionomer (RMGI) (Vitremer, 3M ESPE) to serve as positive control. Restored enamel discs were submitted to 5 days of cariogenic challenge with a microcosm biofilm model. Biofilms were derived from saliva, and grown using a saliva analogue with and without sucrose added intermittently. Outcome variables assessed were the percentage of surface hardness change (\%SHC), the acidogenicity of biofilm supernatants $(\mathrm{pH})$ and microbiological composition of biofilms (CFU counts). Data were analyzed with one-way ANOVA and Tukey's test $(\alpha=0.05)$ for \%SHC and $\mathrm{pH}$, and descriptive statistics for microbiological counts. The $\%$ SHC in enamel was similar within groups treated with the adhesive systems $(\mathrm{p}>0.05)$, which presented higher mineral loss than those restored with RMGI, at 50, 150 and 250 microns from the restoration margin $(\mathrm{p}<0.05)$. The acidogenicity of biofilms was similar within groups ( $p>0.05$ ), and lower lactobacilli counts were found for the AM group. In conclusion, the adhesive systems tested showed no differences in protecting adjacent enamel surfaces against mineral loss when evaluated with an in vitro microcosm biofilm model.

Grant $\mathrm{CNPq}$, Brazilian National Council for Research \# 478885/2008-3 and CAPES-PRODOC \# 420030/0-0.

\section{6 \\ Development of Enamel Lesions by Dental Plaque Microcosm Biofilms in Different Cariogenic Conditions}
L. Angker ${ }^{\mathrm{a}}$, M.V. Swain ${ }^{\mathrm{b},}$ * L. Wong ${ }^{\mathrm{a}}$, C. Sissons ${ }^{\mathrm{a}}$
michael.swain@stonebow.otago.ac.nz
aDental Research Group, Department of Pathology and Molecular Medicine, Wellington School of Medicine and Health Sciences, University of Otago, Wellington, and biomaterials Unit, Department of Oral Rehabilitation, School of Dentistry, University of Otago, Dunedin, New Zealand

The aim was to investigate carious development in tooth enamel induced by plaque microcosm biofilms when cultured under highly cariogenic conditions, and treated with fluoride, casein, and mineralising solution containing calcium-phosphatemonofluorophosphate-urea (CPMU). Ten microcosm biofilms were grown for 28 days on resin-embedded enamel blocks in a Multiplaque Artificial Mouth, and supplied with $10 \%$ sucrose 5 times a day. Half of the plaques were given mineralising solution CPMU (composition: $20 \mathrm{mmol} / 1 \mathrm{CaCl}_{2}, 12 \mathrm{mmol} / \mathrm{l} \mathrm{Na} \mathrm{HPO}_{4}$, $5 \mathrm{mmol} / \mathrm{l} \mathrm{Na}{ }_{2} \mathrm{PO}_{3} \mathrm{~F}$ and $500 \mathrm{mmol} / \mathrm{l}$ urea at $\mathrm{pH} 5.0$, and is supersaturated with respect to hydroxyapatite and fluorapatite) twice a day $(1.5 \mathrm{ml}$ in $6 \mathrm{~min})$ and supplied with either $0,1,000,3,000$ and $5,000 \mathrm{ppm}$ fluoride $(1.5 \mathrm{ml}$ in $6 \mathrm{~min}$ ) or casein $(2 \% \mathrm{w} / \mathrm{v}$ for $30 \mathrm{~min})$ was given twice daily. One of each pair had also CPMU $(15 \mathrm{ml}$ in $6 \mathrm{~min}$ ) twice daily. Plaques supplied with sucrose only, and casein pulses induced the largest lesions: lesion depths (cavitation) were $570 \mu \mathrm{m}(450 \mu \mathrm{m})$ and $410 \mu \mathrm{m}(300 \mu \mathrm{m})$, respectively. CPMU alone substantially decreased enamel demineralisation: lesion depth was $120 \mu \mathrm{m}$. With 1,000 ppm F, only slight surface demineralisation occurred. No lesion could be detected with 5,000 F or when CPMU was added to F-treated plaques. Multiple (3 repeats) SEM observations in backscattered mode and EDAX indicated more severe lesions associated with highly cariogenic plaques with more homogenous demineralisation of the core and walls of enamel rods. Whereas with supply of mineralising solution, enamel lesions had demineralised rods with noticeable remnants of the rod walls with higher mineral content, which were likely to be responsible for preserving a non-cavitated lesion.

The study was supported by a University of Otago Postgraduate Scholarship and the Health Research Council of New Zealand. 


\title{
157
}

\section{Automatic Quantification of Plaque Coverage in vivo for Light-Induced Fluorescence Images}

\author{
E. de Josselin de Jong ${ }^{\mathrm{a}-\mathrm{c}, *}$, M.H. van der Veen ${ }^{\mathrm{b}}$ \\ e.dejosselindejong@inspektor.nl \\ anspektor Research Systems BV, and ${ }^{\mathrm{b}}$ Academic Centre for \\ Dentistry Amsterdam (ACTA), Amsterdam, The Netherlands; \\ 'School of Dental Sciences, University of Liverpool, Liverpool, \\ UK
}

Old plaque on teeth fluoresces bright red. This can be imaged using a QLF-D SLR camera system fitted with an optical filter and blue LEDs (Inspektor Research System BV, Amsterdam). The aim was to develop a fast and reliable algorithm to automatically detect and quantify plaque coverage in-vivo on these QLF-D images. The tooth edges were determined using a Kirsch convolution on intensity levels in RGB color-space at different threshold levels. This resulted in an image with tooth tissue including plaque but excluding gingiva and other background areas. The images were then converted from RGB to HSV color-space to distinguish plaque from clean tooth. The threshold for optimum distinction between tooth and gingiva was determined using 18 images with different levels of plaque coverage taken from the facial aspect of the mouth. Plaque coverage as determined with the algorithm was compared to visual assessment of percentage plaque coverage at each threshold. The level of agreement with visual assessment was highest $(0.99, \mathrm{p}<0.001)$ for a threshold of 1.0. Visual analysis of how the threshold affected tooth area, taught that low thresholds resulted in inclusion of part of the gingiva as tooth area, whereas higher thresholds excluded tooth areas close to the gingiva. A further test on 7 images of subjects with orthodontic brackets proved the algorithm at threshold 1.0 was also suitable on images with orthodontic brackets. Only when brackets were fixed with fluorescing elastic bands did the algorithm fail. In conclusion the new algorithm to determine red fluorescing plaque coverage in QLF-D images proved useful. Its use on images of disclosed plaque needs to be tested. lands.

Inspektor Research Systems BV, Amsterdam, The Nether- 
Abed, Y. 7

Abrams, S.H. 32, 80

Abuhaloob, L. 7

Akimoto, H. 105

Alammari, M.R. 116

Ali, F. 97

Al-Khalifa, K.S. 31

Almeida, B.S. 1

AlShwaimi, E.O. 31

Altenau, A.-C. 30

Altenburger, M.J. 92

Alton, J. 44

Amaechi, B.T. 16, 32, 53

Ambrosano, G. 106

Anderson, C. 34

Anderson, P. 45, 51, 54

Ando, M. 113

Andrian, S. 85

Angker, L. 156

Apel, C. 47

Aranda, W. 77

Arteaga, O. 77

Attin, T. 6, 18, 89

Augustson, D.G. 43

Aumüller, P. 128

Austin, R.S. 90

Azevedo, A. 39

Azevedo, M.S. 155

Azevedo, R.B. 117

Bachanek, T. 13

Badea, M. 119

Baelum, V. 120

Bakht, K. 35, 57

Baltisberger, A. 110

Bangdiwala, S. 36

Barbour, M.E. 50, 51, 82

Barr, R. 34

Bärsch, N. 92

Bartlett, D.W. 90

Basso, T.T. 140

Battancs, E. 62

Batterham, A.M. 8, 10

Bautista, G. 104

Beecher, T. 15

Beighton, D. 126

Bertaud, V. 79

Berthold, L. 98

Berzina, S. 145
Bilgin, G. 59

Bitter, K. 22

Björklund, M. 132

Blair, Y. 73-75, 137

Bonecker, M. 94

Borutta, A. 145

Bottenberg, P. 37

Bratanow, M. 124

Brighenti, F.L. 134, 135

Brinkmane, A. 80

Brockmann, E. 127

Bronkhorst, E.M. 40, 67

Brovka, D. 71

Brown, S. 34

Buchalla, W. 18

Bücher, K. 111

Buijs, M.J. 130

Burnett, G. 35, 57

Busche, J. 103

Bussaneli, D. 107

Buzalaf, M.A.R. 1, 9, 14, 84, 94

Cabello, R. 36, 76, 77, 122

Cagetti, M.G. 140

Callaway, A. 124

Campus, G. 66, 140

Cankar, K. 115

Care, R. 145

Carvajal, P. 77

Carvalho, A.C. 65

Carvalho, J.C. 147

Carvalho, R. 117

Carvalho, T.S. 94

Castaño-Duque, S.P. 104

Ccahuana, R.A. 125

Cenci, M.S. 155

Cerezetti, R.V. 4

Chalas, R. 13

Charadram, N. 60

Chavarra, N. 150

Chedjieu, I. 16, 53

Chen, X. 25

Chew, H.P. 100, 102

Choi, C.H. 55

Choi, Y.H. 151

Chouljian, 32

Christiansen, J. 68, 69

Christiansen, M.E.C. 68, 69

Chung, S.Y. 151
Churchley, D. 44

Cismak, A. 61, 98

Cochrane, N.J. 54

Coelho, C. 38, 39

Collins, B.D. 59

Congiu, G. 66

Conrads, G. 47

Conway, D.I. 137

Cooper, L. 48

Cordeiro, R.C.L. 106, 107

Corvino, M. 14

Costa-Silva, C.M. 106

Crielaard, W. 130, 131

Crombie, F.A. 46

Cronin, M. 78

Culshaw, S. 137

Cury, J.A. 2-4, 125

Davies, M. 102

Davis, G.R. 54

de Josselin de Jong, E. 35, 57, 114, 116, 138, 157

de Moura, V. 145

de Moura-Sieber, R. 145

de Paula, L.M. 40a

de Santana Giongo, F.C.M. 28

de Soet, J.J. 138

Decker, M. 96

Decourt, R. 40a

Del Bel Cury, A.A. 2-4

Delbem, A.C.B. 134, 135

Deng, D.M. 131

Díaz, C. 36

Dige, I. 127

Diniz, M.B. 107, 143

Doi, T. 33

Dörfer, C. 56

Dorozhkin, S.V. 41

Du, M.Q. 25

Duckworth, R. 8

Duggal, M.S. 5, 97

Duran, K. 150

Duran, L. 150

Ebisu, S. 144

Eckert, G. 113

Eduardo, C.P. 47

Ehrlich, B.R. 32

Ekstrand, K.R. 68, 69, 150
El Banna, M. 27, 49, 139

Eller, J. 62

Ellwood, R. 121

Ellwood, R.P. 100, 102, 153

Elman, G.I. 32

Elmazi, V. 6

El Zayat, I.M.G. 27, 49, 139

Erichsen, M.M. 30

e Silva, B.B. 28

Espinoza, I. 77

Esteves-Oliveira, M. 4

Faleiros, S. $36,76,77,122$

Falla, S. 81

Fan, M.W. 25

Fernandes, J.K.B. 2

Ferreira, F.M. 65

Festy, F. 90

Finer, Y. 32

Fischer, B. 109

Flanigan, P.J. 17

Flannigan, N.L. 48

Fonseca, J.A.C. 147

Forssten, S.D. 132

Frankenberger, R. 103

Freitas, L.S.F. 134

Frencken, J.E. 25, 67

Fujiki, S. 105

Fujita, K. 129

Fukushima, R. 1, 9

Gakunga, P.T. 53

Galeone, C. 140

Galler, M. 111

Gamonal, J. 77

Ganss, C. 86, 87, 99

Gatica, H. 36

Georgescu, A. 85

Giacaman, R.A. 125

Gilbert, S.C. 126

Girondi, C.M. 135

Gomes, R.R.C. 147

Gomez, J. 81, 121

Goodwin, M. 153

Gorbatova, L. 63

Gorbatova, M. 63

Gordillo, S.M. 150

Gorzó, I. 62

Gracia, L.H. 82

\section{KARGER}

๑) 2011 S. Karger AG, Basel

Fax +41 613061234

E-Mail karger@karger.ch

www.karger.com 
Grannell, A. 15

Gresnigt-Bekker, C.O.V.M. 23

Grguraš, N. 146

Grizzo, L.T. 14

Groisman, S. 14

Gudkina, J. 80

Günday, M. 59

Guzman, C. 122

Haak, R. 109

Hammad, S.M. 27, 49

Han, D.H. 72

Hannig, C. 86

Hara, A. 44

Harding, M. 78

Hatch, J.P. 53

Hayashi, M. 105, 144

Hayran, O. 59

Hector, M.P. 45

Hegedüs, C. 88

Heinrich, J. 29

Heinzel-Gutenbrunner, M. 24 103

Hellen, A. 32

Hellen, W.M.P. 32

Hellwig, E. 92

Henriques, M. 38, 39

Hernandez-Zambrano, M.D. 104

Hickel, R. 29, 111

Higham, S.M. 34, 35, 48, 57, 58, 116, 133

Hjortsjö, C. 91, 98

Höfling, J.F. 136

Holme, B. 95

Hong, S.J. 55

Hoogstraten, J. 149

Hopcraft, M. 112

Hope, C.K. 35, 48, 57

Hove, L.H. 90, 95

Hug, I. 110, 143

Hunter, N. 60

Husebye, E.S. 30

Huysman, M.C.D.N.J.M. 25, 40, 155

Iijima, Y. 42

Imfeld, T. 6

Inaba, D. 114

Inagaki, S. 129

Iovan, G. 85

Ito, A. 144

Ito, N. 84

Jablonski-Momeni, A. 24, 103

Jalili, J. 50

Jan, J. 146

Jardim, J.J. 40a

Jeon, R.J. 32

Jeong, S.S. 55
Jeremias, F. 106

Jin, K. 33

Jones, S.B. 50, 100, 101, 102

Jonski, G. 91

Jonsson, M.V. 30

Jun, E.J. 72

Jung, S.H. 141

Jung, Y.S. 151

Juric, H. 21

Kamasaka, H. 19

Kambara, M. 33

Kargül, B. 59

Karlinsey, R.L. 20

Karlsson, L. 143

Kato, K. 154

Kaur, R. 126

Kawasaki, K. 33

Kiesow, A. 61

Kilmer, S. 17

Kim, B.-I. 114

Kim, B.I. 141

Kim, J.B. 72

Kim, M.J. 72

Kingston, R. 15

Kiso, Y. 129

Klimek, J. 86, 87, 99

Kneist, S. 124, 145

Kneist, S. 96

Kobayashi, T. 19

Koga-Ito, C.Y. 134, 135

Kókai, E.L. 62

Komarov, G.N. 34

Kontani, M. 129

Korkut, B. 59

Kosoric, J. 45

Kuczumow, A. 13

Kühnisch, J. 29, 111

Kumar, N.C. 43

Kunzelmann, K.H. 111

Kuper, N.K. 40

Küpper, H. 96, 124

Kuriki, T. 19

Kurokawa, A. 148

Kusano, S.C. 3

Kuyumdjidi, N.V. 70

Kwon, H.K. 141

Lampert, F. 47

Landes, D. 12

Lara, J.S. 104

Leal, S.C. 67,117

Lee, H.J. 55

Lee, S.M. 72

Lesquibe-Perrus, C. 79

Levy, F.M. 1

Li, X.L. 131

Lima, L.M. 107

Ling, J.Q. 131

Lingawi, H. 51
Lingström, P. 140

Lippert, F. 44

Lloyd, J. 12

Locane, G. 80

Loivos, C. 14

Lourenço, C. 142

Lugliè, P.F. 66

Lund, R.G. 155

Lussi, A. 83, 94, 108, 110, 143

Lynch, R.J.M. 44, 51, 58

Macdonald, E.L. 101

Mach, D. 29

Machiulskiene, V. 120

Mackey, A.C. 20

Macpherson, L.M.D. 73-75, 137

Madléna, M. 88

Maeder, F. 110

Magalhães, A.C. 84

Maguire, A. 8, 10, 12, 153

Maiorini, E. 66

Maki, Y. 148

Malcolm, J. 137

Malinowski, M. 5

Maltz, M. 28, 40a

Mandelis, A. 32

Manton, D.J. 46

Margraf-Stiksrud, J. 24

Marin, L. 81

Marthaler, T. 11

Marthinussen-Cuida, M. 30

Martignon, S. 81, 104

Martin, G.C. 35, 57

Márton, S. 88

Maslak, E.E. 70, 145

Mastnak, M. 146

Matsumoto-Nakano, M. 123, 129

Mattos-Graner, R.O. 136

McCall, D.R. 73, 75

McGarrell, B. 53

McGrady, M.G. 153

McMahon, A.D. 73, 75

Megert, B. 83

Melo, P. 38, 39

Mendoza, C. 77

Mensinkai, P. 53

Mestrinho, H.D. 40a, 147

Meyer, R.L. 127

Meyer-Lueckel, H. 22, 56

Mialhe, F. 106

Miyake, T. 33

Moazzez, R. 90

Mohsen, M.M.A. 27

Molyneux, L.E. 48

Monert, L. 99

Mora, I. 104

Morawietz, M. 61

Moron, B.M. 84
Moynihan, P. 12

Mua, B. 28

Mulder, J. 25

Muñoz, A. 77

Muñoz, C. 125

Muñoz, M.J. 125

Mutti, B. 20

Nagayama, K. 129

Nagy, G. 88

Naka, S. 123

Nakagaki, H. 154

Nakano, K. 123

Nemeth, L. 115

Neuhaus, K.W. 110, 143

Nomura, R. 123

Novák, T. 62

Nowak, J. 13

Nyvad, B. 120, 127

O'Hare, W.T. 8, 10

Oh, H.N. 55

Olival, A.R. 14

Oliveira, M.A.C. 134

Oliveira, T.J. 4

Omid, N. 8, 10, 12

Ooshima, T. 123, 129

Ouwehand, A.C. 132

Owens, G.J. 58, 133

Paiva, S.M. 65

Pál, A. 62

Palamara, J. 46

Palasti Kovacs, B. 88

Paris, S. 22, 56

Park, K. 109

Pastbin, M. 63

Pastbina, I. 63

Patel, D. 153

Pender, N. 48

Pender, S.M. 48

Peric, T. 52

Pessan, J.P. 1, 9

Petrovic, B. 52

Petzold, M. 61, 98

Pfarrer, A.M. 17, 43

Pieper, K. 24, 103

Pollheim, H. 47

Pordeus, I.A. 65

Prentic Bakic, S. 21

Pretty, I.A. 100, 121, 153

Purger, F. 108

Pustavoitava, N. 118, 119, 120

Qvist, V. 28

Radnai, M. 62

Ramage, G. 137

Ramalingam, K. 16, 53

Rangel, M.C. 81 
Ravn, I. 127

Reinhoefer, N. 96

Reynolds, E.C. 46, 54

Ribeiro, A. 108

Ricci-Nittel, D. 20

Richard, F. 79

Rodionova, A.S. 70

Rodrigues, J.A. 108, 110, 143

Rodrigues, M.H.C. 1

Rodríguez, G. 36, 76, 77, 122

Rogovska, I. 80

Roos, M. 89

Rosado, L. 119

Rotgans, J. 11

Ruiz, J.A. 81

Rusak, A. 71

Rusin, R.P. 43

Sakagami, T. 113

Salsone, S. 121

Salvador, M.J. 134, 135

Sampaio, F.C. 1, 9, 94

Sanchez, J. 122

Sandu, A.V. 85

Santamaria, R.M. 68, 69

Santos-Pinto, L. 106, 107

Santrain, M.V. 142

Sarembe, S. 61

Satie, S. 84

Saxegaard, E. 91

Scarpelli, A.C. 65

Schellwien, T. 56

Schemeron, B.R. 59

Schiffner, U. 128

Schlueter, N. 86, 87, 99

Schneider, H. 109

Schneider, S. 89

Schreiber, A. 11

Schulte, A.G. 37

Schulze, K. 87
Seitz, M. 111

Senderovitz, F. 68, 69

Sener, B. 6, 18, 89

Senna, A. 140

Serša, I. 115

Shabunko, D. 71, 93

Shah, S. 45

Shakavets, N. 26

Shakovets, N. 145

Sharma, D. 20

Sheehy, E. 126

Shellis, R.P. 83

Sheriff, A. 137

Shkarin, V.V. 70

Silva, E.F. 147

Silva, M.J. 39

Silvertown, J.D. 32

Sissons, C. 156

Sivagurunathan, K. 32

Skrivele, S. 145

Smith, P.W. 34, 48, 116

Soet, J.J. 14

Song, H.J. 55

Song, K.B. 151

Sono, R. 42

Souza, J.F. 106

Souza, P.R. 108

Soviero, V.M. 117

Spano, G. 66

Splieth, C.H. 64

Stacey, M. 112

Steen, N. 12

Stein, S. 24

Stenhagen, K.R. 90, 95

Stich, H. 143

Stipp, R.N. 136

Stoleriu, S. 85

Stookey, G. 59

Strafford, S. 5

Strohmenger, L. 140
Sugiyama, S. 105

Swain, M. 60

Swain, M.V. 156

Tağtekin, D. 59

Takashima, Y. 129

Takatsuka, T. 42

Takii, H. 19

Tamura, K. 154

Tanaka, T. 19

Tantbirojn, D. 43

Tauböck, T.T. 18

Taylor, A. 121

Teasdale, L. 12

Tellez, M. 81

ten Cate, J.M. 130, 138

Tenuta, L.M.A. 2-4

Thiering, E. 29

Thusu, A. 16

Tikhonova, S. 118, 120

Toumba, K.J. 5, 97

Tserakhava, T. 26, 145

Tveit, A.B. 90, 95

Uene, M. 33

Urzua, I. 36, 76, 77, 122

Valappil, S.P. 58, 133

van de Sande, F.H. 155

van der Veen, M.H. 114, 138, 157

van Loveren, C. 23, 149

van Strijp, A.J.P. 42

Vasconcellos, A.B. 108

Verhoef, A. 23

Vermaire, J.H. 149

Véronneau, J. 118, 119, 152

Versluis, A. 43

Vidmar, J. 115

Viegas, C.M. 65
Vieira, A.P.G.F. 142

Vilhena, F. 84

Vlasova, D.S. 70

Volgenant, C.M.C. 138

von Hinckeldey, J. 99

Wagner, M. 145

Walls, R. 12

Wang, X. 83

Weber, K. 24

Wegehaupt, F.J. 6

West, N.X. 50, 100, 101, 102

Weuste, M. 109

Wheeler, J. 101

Whelton, H. 15

White, A.J. 82

Whitford, G.M. 1

Wiegand, A. 89

Willershausen, B. 124

Williams, J.S. 59

Wong, L. 156

Wrbas, K.-T. 92

Yamada, K. 148

Yanıkoğlu, F. 59

Yanovskaya, M.L. 70

Young, A. 91, 98

Youngson, C.C. 48

Yudina, N.A. 71, 93

Yuris, O. 71

Zago, C.E. 107

Zakian, C.M. 100, 102, 121

Zaura, E. 130

Zero, D. 113

Zohoori, F.V. 8, 10, 12

Zsigmond, A. 88

Zuanon, A.C.C. 106 